Supporting Information

\title{
Synthesis and Reactivity of 3,5-Diiodo-BODIPYs via a Concerted, Double Aromatic Finkelstein Reaction
}

Felicity Frank, Paul G. Waddell, Michael J. Hall* and Julian G. Knight*

Chemistry, School of Natural and Environmental Sciences, Newcastle University, Newcastle Upon Tyne, NE1 7RU, UK. 


\section{Supporting Information}

General experimental information

\section{Experimental procedures}

Methyl 4-(5,5-difluoro-3,7-diiodo-5H-4 $\lambda^{4}, 5 \lambda^{4}$-dipyrrolo[1,2-c:2', $1^{\prime}$ -

$f][1,3,2]$ diazaborinin-10-yl)benzoate (3a)

5,5-difluoro-3,7-diiodo-10-(3-nitrophenyl)-5H-4 $\lambda^{4}, 5 \lambda^{4}$-dipyrrolo[1,2$\left.c: 2^{\prime}, 1^{\prime}-f\right][1,3,2]$ diazaborinine $(\mathbf{3 b})$

5,5-difluoro-3,7-diiodo-10-(4-methoxyphenyl)-5H-4 $\lambda^{4}, 5 \lambda^{4}$-dipyrrolo[1,2$\left.c: 2^{\prime}, 1^{\prime}-f\right][1,3,2]$ diazaborinine $(\mathbf{3 c})$

5,5-difluoro-3,7-diiodo-10-(3-methoxyphenyl)-5H-4 $\lambda^{4}, 5 \lambda^{4}$-dipyrrolo[1,2$\left.c: 2^{\prime}, 1^{\prime}-f\right][1,3,2]$ diazaborinine (3d)

Methyl 4-(5,5-difluoro-3,7-diphenyl-5H-4 $\lambda^{4}, 5 \lambda^{4}$-dipyrrolo[1,2-c:2', $1^{\prime}-$

$f][1,3,2]$ diazaborinin-10-yl)benzoate (4) and methyl 4-(3-butyl-

5,5-difluoro-7-phenyl-5H-5 $\lambda^{4}, 6 \lambda^{4}$-dipyrrolo[1,2-c:2', $1^{\prime}-$

$f][1,3,2]$ diazaborinin-10-yl)benzoate (5)

Methyl 4-(5,5-difluoro-3,7-di((E)-styryl)-5H-4 $\lambda^{4}, 5 \lambda^{4}$-dipyrrolo[1,2-

$\left.c: 2^{\prime}, 1^{\prime}-f\right][1,3,2]$ diazaborinin-10-yl)benzoate (6)

Methyl 4-(5,5-difluoro-3,7-bis(phenylethynyl)-5H-4 $\lambda^{4}, 5 \lambda^{4}$-dipyrrolo[1,2$c: 2$ ',1'-f][1,3,2]diazaborinin-10-yl)benzoate (7) and methyl 4-(3chloro-5,5-difluoro-7-(phenylethynyl)-5H-4 $\lambda^{4}, 5 \lambda^{4}$-dipyrrolo[1,2$\left.c: 2^{\prime}, 1^{\prime}-f\right][1,3,2]$ diazaborinin-10-yl)benzoate (8)

Methyl 4-(5,5-difluoro-3,7-diphenyl-5H-4 $\lambda^{4}, 5 \lambda^{4}$-dipyrrolo[1,2-c:2', $1^{\prime}-$ $f][1,3,2]$ diazaborinin-10-yl)benzoate (4) and methyl 4-(3-chloro5,5-difluoro-7-phenyl-5H-5 $\lambda^{4}, 6 \lambda^{4}$-dipyrrolo[1,2-c:2',1'$f][1,3,2]$ diazaborinin-10-yl)benzoate (9)

Solubility Measurements for $\mathrm{NaCl}, \mathrm{NaBr}$ and $\mathrm{NaI}$ in Propionitrile 


\section{Single crystal $X$-ray data and structure refinement}

3,6-dibromo-5,5-difluoro-9-(4-methoxyphenyl)-5H-5 $\lambda^{4}$ -

cyclopenta[c]pyrrolo[2,1-f][1,2]azaborinine) (2c)

3,6-dibromo-5,5-difluoro-9-(3-methoxyphenyl)-5H-5 $\lambda^{4}$ -

S46

cyclopenta[c]pyrrolo[2,1-f][1,2]azaborinine (2d)

Methyl 4-(5,5-difluoro-3,7-diiodo-5H-4 $\lambda^{4}, 5 \lambda^{4}$-dipyrrolo[1,2-c:2',1'-

S48

$f][1,3,2]$ diazaborinin-10-yl)benzoate (3a)

5,5-difluoro-3,7-diiodo-10-(4-methoxyphenyl)-5H-4 $\lambda^{4}, 5 \lambda^{4}$-dipyrrolo[1,2$\left.c: 2^{\prime}, 1^{\prime}-f\right][1,3,2]$ diazaborinine $(\mathbf{3 c})$

5,5-difluoro-3,7-diiodo-10-(3-methoxyphenyl)-5H-4 $\lambda^{4}, 5 \lambda^{4}$-dipyrrolo[1,2$\left.c: 2^{\prime}, 1^{\prime}-f\right][1,3,2]$ diazaborinine (3d)

Methyl 4-(5,5-difluoro-3,7-diphenyl-5H-4 $\lambda^{4}, 5 \lambda^{4}$-dipyrrolo[1,2-c:2', $1^{\prime}-$

$f][1,3,2]$ diazaborinin-10-yl)benzoate (4)

Methyl 4-(5,5-difluoro-3,7-di((E)-styryl)-5H-4 $\lambda^{4}, 5 \lambda^{4}$-dipyrrolo[1,2-

S56

$\left.c: 2^{\prime}, 1^{\prime}-f\right][1,3,2]$ diazaborinin-10-yl)benzoate (6)

\section{References}

\section{NMR Spectra $\left({ }^{1} \mathrm{H},{ }^{13} \mathrm{C},{ }^{19} \mathrm{~F}\right.$ and $\left.{ }^{11} \mathrm{~B}\right)$}

Methyl 4-(5,5-difluoro-3,7-diiodo-5H-4 $\lambda^{4}, 5 \lambda^{4}$-dipyrrolo[1,2-c:2', $1^{\prime}-$

$f][1,3,2]$ diazaborinin-10-yl)benzoate (3a)

5,5-difluoro-3,7-diiodo-10-(3-nitrophenyl)-5H-4 $\lambda^{4}, 5 \lambda^{4}$-dipyrrolo[1,2$\left.c: 2^{\prime}, 1^{\prime}-f\right][1,3,2]$ diazaborinine (3b)

5,5-difluoro-3,7-diiodo-10-(4-methoxyphenyl)-5H-4 $\lambda^{4}, 5 \lambda^{4}$-dipyrrolo[1,2$\left.c: 2^{\prime}, 1^{\prime}-f\right][1,3,2]$ diazaborinine $(\mathbf{3 c})$

5,5-difluoro-3,7-diiodo-10-(3-methoxyphenyl)-5H-4 $\lambda^{4}, 5 \lambda^{4}$-dipyrrolo[1,2$\left.c: 2^{\prime}, 1^{\prime}-f\right][1,3,2]$ diazaborinine (3d)

Methyl 4-(5,5-difluoro-3,7-diphenyl-5H-4 $\lambda^{4}, 5 \lambda^{4}$-dipyrrolo[1,2-c:2', $1^{\prime}-$

$f][1,3,2]$ diazaborinin-10-yl)benzoate (4)

Methyl 4-(3-butyl-5,5-difluoro-7-phenyl-5H-5 $\lambda^{4}, 6 \lambda^{4}$-dipyrrolo[1,2-c:2',1'$f][1,3,2]$ diazaborinin-10-yl)benzoate (5) 
Methyl 4-(5,5-difluoro-3,7-di((E)-styryl)-5H-4 $\lambda^{4}, 5 \lambda^{4}$-dipyrrolo[1,2-

$\left.c: 2^{\prime}, 1^{\prime}-f\right][1,3,2]$ diazaborinin-10-yl)benzoate (6)

Methyl 4-(5,5-difluoro-3,7-bis(phenylethynyl)-5H-4 $\lambda^{4}, 5 \lambda^{4}$-dipyrrolo[1,2-

$\left.c: 2^{\prime}, 1^{\prime}-f\right][1,3,2]$ diazaborinin-10-yl)benzoate (7)

Methyl 4-(3-chloro-5,5-difluoro-7-(phenylethynyl)-5H-4 $\lambda^{4}, 5 \lambda^{4}$ -

S91

dipyrrolo[1,2-c:2',1'-f][1,3,2]diazaborinin-10-yl)benzoate (8)

Methyl 4-(3-chloro-5,5-difluoro-7-phenyl-5H-5 $\lambda^{4}, 6 \lambda^{4}$-dipyrrolo[1,2-

S95

$\left.c: 2^{\prime}, 1^{\prime}-f\right][1,3,2]$ diazaborinin-10-yl)benzoate (9)

\section{High Resolution Mass Spectrometry}

Methyl 4-(5,5-difluoro-3,7-diiodo-5H-4 $\lambda^{4}, 5 \lambda^{4}$-dipyrrolo[1,2-c:2', $1^{\prime}-$

$f][1,3,2]$ diazaborinin-10-yl)benzoate (3a)

5,5-difluoro-3,7-diiodo-10-(3-nitrophenyl)-5H-4 $\lambda^{4}, 5 \lambda^{4}$-dipyrrolo[1,2$\left.c: 2^{\prime}, 1^{\prime}-f\right][1,3,2]$ diazaborinine $(\mathbf{3 b})$

5,5-difluoro-3,7-diiodo-10-(4-methoxyphenyl)-5H-4 $\lambda^{4}, 5 \lambda^{4}$-dipyrrolo[1,2$\left.c: 2^{\prime}, 1^{\prime}-f\right][1,3,2]$ diazaborinine $(\mathbf{3 c})$

5,5-difluoro-3,7-diiodo-10-(3-methoxyphenyl)-5H-4 $\lambda^{4}, 5 \lambda^{4}$-dipyrrolo[1,2$\left.c: 2^{\prime}, 1^{\prime}-f\right][1,3,2]$ diazaborinine $(\mathbf{3 d})$

Methyl 4-(5,5-difluoro-3,7-diphenyl-5H-4 $\lambda^{4}, 5 \lambda^{4}$-dipyrrolo[1,2-c:2', $1^{\prime}-$

$f][1,3,2]$ diazaborinin-10-yl)benzoate (4)

Methyl 4-(3-butyl-5,5-difluoro-7-phenyl-5H-5 $\lambda^{4}, 6 \lambda^{4}$-dipyrrolo[1,2-c:2',1'-

S102

$f][1,3,2]$ diazaborinin-10-yl)benzoate (5)

Methyl 4-(5,5-difluoro-3,7-di((E)-styryl)-5H-4 $\lambda^{4}, 5 \lambda^{4}$-dipyrrolo[1,2-

S103 $\left.c: 2^{\prime}, 1^{\prime}-f\right][1,3,2]$ diazaborinin-10-yl)benzoate (6)

Methyl 4-(5,5-difluoro-3,7-bis(phenylethynyl)-5H-4 $\lambda^{4}, 5 \lambda^{4}$-dipyrrolo[1,2-

$\left.c: 2^{\prime}, 1^{\prime}-f\right][1,3,2]$ diazaborinin-10-yl)benzoate (7)

Methyl 4-(3-chloro-5,5-difluoro-7-(phenylethynyl)-5H-4 $\lambda^{4}, 5 \lambda^{4}$ dipyrrolo[1,2-c:2',1'-f][1,3,2]diazaborinin-10-yl)benzoate (8)

Methyl 4-(3-chloro-5,5-difluoro-7-phenyl-5H-5 $\lambda^{4}, 6 \lambda^{4}$-dipyrrolo[1,2$\left.c: 2^{\prime}, 1^{\prime}-f\right][1,3,2]$ diazaborinin-10-yl)benzoate (9) 


\section{General experimental information}

${ }^{1} \mathrm{H},{ }^{13} \mathrm{C},{ }^{11} \mathrm{~B}$ and ${ }^{19} \mathrm{~F}$ NMR spectra were recorded directly with a Jeol Lambda $500 \mathrm{MHz}$, Jeol ECS-400 MHz or Brucker Avance $300 \mathrm{MHz}$. HRMS data were provided by the EPSRC National Mass Spectrometry Service (University of Swansea). IR spectra were obtained as neat samples using a Varian 800 FT-IR Scimitar Series spectrometer scanning from 4000-600 cm 1. UV-Vis spectra were obtained using a UV-1800 Shimadzu UV spectrophotometer scanning from $300-700 \mathrm{~nm}$. Fluorescence quantum yield spectra were obtained using Shimadzu RF6000 spectrofluorophotometer. Fluorescence quantum yield data was obtained against a standard reference of either Rhodamine 6G or Cresyl violet, 99\% pure, laser grade. 3,5dichloro-BODIPYs (1a-d) and 3,5-dibromochloro-BODIPYs (2a-d) were prepared according to the literature.[S1] 
Methyl 4-(5,5-difluoro-3,7-diiodo-5H-4 $\lambda^{4}, 5 \lambda^{4}$-dipyrrolo[1,2-c:2',1'-f][1,3,2]diazaborinin10-yl)benzoate (3a)

\section{Method A:}

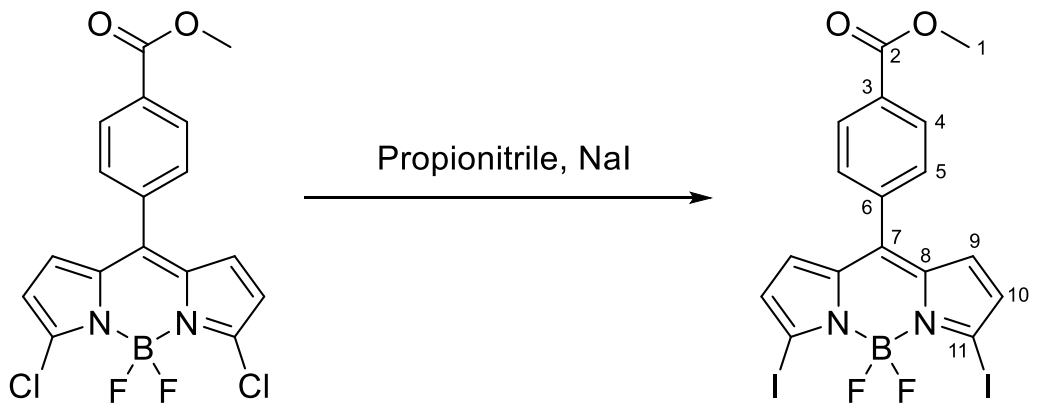

To a $25 \mathrm{~mL}$ round bottom flask was added methyl 4-(3,7-dichloro-5,5-difluoro- $5 H-4 \lambda^{4}, 5 \lambda^{4}$ dipyrrolo[1,2-c:2',1'-f][1,3,2]diazaborinin-10-yl)benzoate (50 mg, $0.13 \mathrm{mmol})$ and saturated $\mathrm{NaI}$ solution $(0.8 \mathrm{~mL})$. The reaction mixture was refluxed, using an oil bath, for 24 hours then diluted with DCM (10 mL). The organic layer was washed with $\mathrm{H}_{2} \mathrm{O}(2 \times 10 \mathrm{~mL})$, dried over $\mathrm{Na}_{2} \mathrm{SO}_{4}$, filtered and the solvent was removed under reduced pressure to give a dark purple solid. The crude product was purified through silica gel column chromatography (1:2 Petrol : DCM) to give methyl 4-(3,7-diiodo-5,5-difluoro-5H-4 $\lambda^{4}, 5 \lambda^{4}$-dipyrrolo[1,2-c:2', $1^{\prime}$ $f][1,3,2]$ diazaborinin-10-yl)benzoate (66 $\mathrm{mg}, 0.12 \mathrm{mmol}, 95 \%)$ as a purple solid.

\section{Method B:}
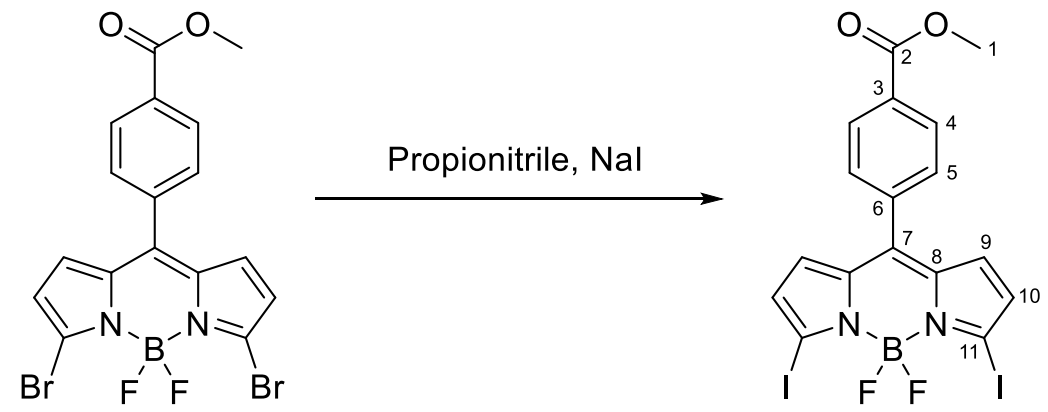

To a $25 \mathrm{~mL}$ round bottom flask was added methyl methyl 4-(3,7-dibromo-5,5-difluoro-5H$4 \lambda^{4}, 5 \lambda^{4}$-dipyrrolo[1,2-c:2',1'-f][1,3,2]diazaborinin-10-yl)benzoate (1.0 g, $\left.2.07 \mathrm{mmol}\right)$ and saturated $\mathrm{NaI}$ solution $(30 \mathrm{~mL})$. The reaction mixture was refluxed, using an oil bath, for 1.5 hours then diluted with DCM $(100 \mathrm{~mL})$. The organic layer was washed with $\mathrm{H}_{2} \mathrm{O}(2 \times 100 \mathrm{~mL})$, dried over $\mathrm{Na}_{2} \mathrm{SO}_{4}$, filtered and the solvent was removed under reduced pressure to give a purple solid. The crude product was purified through silica gel column chromatography (DCM) 
to give methyl 4-(5,5-difluoro-3,7-diiodo-5H-4 $\lambda^{4}, 5 \lambda^{4}$-dipyrrolo[1,2-c:2', $1^{\prime}$ $f][1,3,2]$ diazaborinin-10-yl)benzoate $(1.07 \mathrm{~g}, 1.85 \mathrm{mmol}, 90 \%)$ as a purple solid.

Methyl 4-(5,5-difluoro-3,7-diiodo-5 $H-4 \lambda^{4}, 5 \lambda^{4}$-dipyrrolo[1,2-c:2', $\left.1^{\prime}-f\right][1,3,2]$ diazaborinin10-yl)benzoate (3a)

Rf: 0.85 (DCM). Mp: $156-157^{\circ} \mathrm{C} .{ }^{1} \mathbf{H}$ NMR $\left(300 \mathrm{MHz}, \mathrm{CDCl}_{3}\right) \delta 8.17\left(\mathrm{~d}, J=8.4 \mathrm{~Hz}, 2 \mathrm{H}, \mathrm{H}^{4}\right)$, $7.57\left(\mathrm{~d}, J=8.4 \mathrm{~Hz}, 2 \mathrm{H}, \mathrm{H}^{5}\right), 6.72\left(\mathrm{~d}, J=4.2 \mathrm{~Hz}, 2 \mathrm{H}, \mathrm{H}^{9}\right), 6.61\left(\mathrm{~d}, J=4.2 \mathrm{~Hz}, 2 \mathrm{H}, \mathrm{H}^{10}\right), 3.98$ (s, 3H, $\left.\mathrm{H}^{1}\right) .{ }^{13} \mathrm{C}$ NMR $\left(75 \mathrm{MHz}\right.$, Chloroform- $d$ ) $\delta 166.2\left(\mathrm{C}^{2}\right), 139.7\left(\mathrm{C}^{7}\right), 137.8\left(\mathrm{C}^{8}\right), 136.9$ $\left(\mathrm{C}^{3}\right), 132.3\left(\mathrm{C}^{6}\right), 131.6\left(\mathrm{C}^{10}\right), 130.6-130.4\left(\mathrm{~m}, \mathrm{C}^{9}\right), 130.4\left(\mathrm{C}^{5}\right), 129.8\left(\mathrm{C}^{4}\right), 104.0\left(\mathrm{C}^{11}\right), 52.7$ $\left(\mathrm{C}^{l}\right) .{ }^{19} \mathbf{F}$ NMR $(282 \mathrm{MHz}$, Chloroform- $d$ ) $\delta-144.75$ (q, $J=29.5 \mathrm{~Hz}) .{ }^{11} \mathbf{B}$ NMR $(96 \mathrm{MHz}$, Chloroform- $d$ ) $\delta 0.72$ (t, $J=30.1 \mathrm{~Hz}$ ). IR (neat): $\mathrm{V}_{\max } / \mathrm{cm}^{-1} 3107(\mathrm{C}-\mathrm{H}, \mathrm{w}), 1707(\mathrm{C}=\mathrm{O}, \mathrm{w})$. HRMS: (ASAP+) calcd for $\mathrm{C}_{17} \mathrm{H}_{11} \mathrm{BI}_{2} \mathrm{~F}_{2} \mathrm{~N}_{2} \mathrm{O}_{2}[\mathrm{M}-\mathrm{F}]^{+}:$558.8990, found 558.8995. UV-Vis: $\lambda_{\max }=545 \mathrm{~nm}(\mathrm{DCM})$. Molar extinction coefficient $(\varepsilon)=63,000 \mathrm{M}^{-1} \mathrm{~cm}^{-1} \cdot \phi_{\mathrm{F}}: 0.13$ 


\section{5,5-difluoro-3,7-diiodo-10-(3-nitrophenyl)-5H-4 $\lambda^{4}, 5 \lambda^{4}$-dipyrrolo[1,2-c:2',1'-}

\section{$f][1,3,2]$ diazaborinine $(3 b)$}

\section{Method A:}
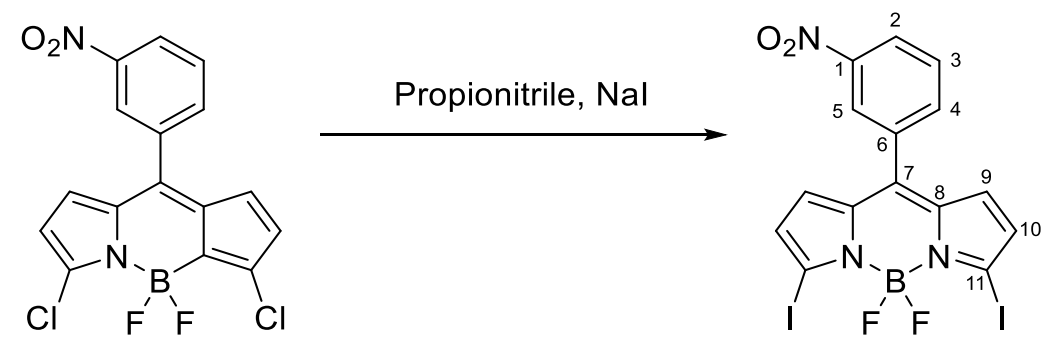

To a $25 \mathrm{ml}$ round bottom flask was added methyl 3,7-dichloro-5,5-difluoro-10-(3-nitrophenyl)$5 H-4 \lambda^{4}, 5 \lambda^{4}$-dipyrrolo[1,2-c:2', $\left.1^{\prime}-f\right][1,3,2]$ diazaborinine $(43 \mathrm{mg}, 0.13 \mathrm{mmol}$ ) and saturated $\mathrm{NaI}$ solution $(2 \mathrm{ml})$. The reaction mixture was refluxed, using an oil bath, for 6 hours then diluted with DCM (10 ml). The organic layer was washed with $\mathrm{H}_{2} \mathrm{O}(2 \times 40 \mathrm{ml})$, dried over $\mathrm{Na}_{2} \mathrm{SO}_{4}$, filtered and the solvent was removed under reduced pressure to give a purple solid. The crude product was purified through silica gel column chromatography (1:1 Petrol:ethyl acetate) to give 5,5-difluoro-3,7-diiodo-10-(3-nitrophenyl)-5H-4 $\lambda^{4}, 5 \lambda^{4}$-dipyrrolo[1,2-c:2',1'$f][1,3,2]$ diazaborinine $(59.2 \mathrm{mg}, 0.10 \mathrm{mmol} 93 \%)$ as a pink solid.

\section{Method B:}
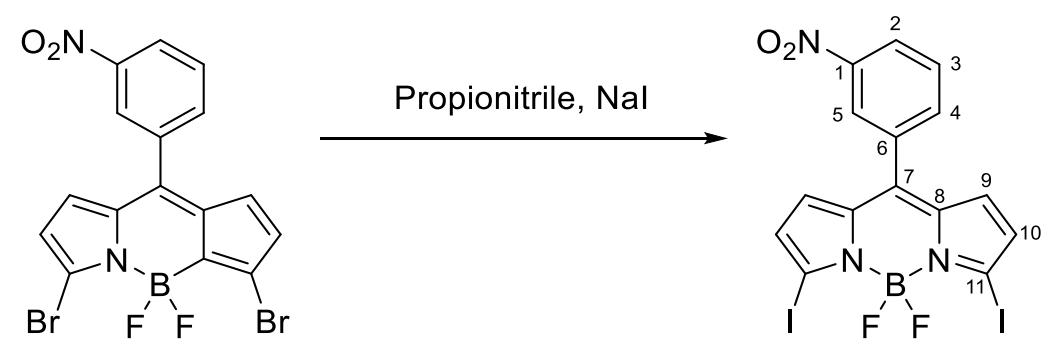

To a $25 \mathrm{~mL}$ round bottom flask was added methyl 3,7-dibromo-5,5-difluoro-10-(3nitrophenyl)-5H-4 $4 \lambda^{4}, 5 \lambda^{4}$-dipyrrolo[1,2-c:2',1'-f][1,3,2]diazaborinine (63.2 $\left.\mathrm{mg}, 0.13 \mathrm{mmol}\right)$ and saturated $\mathrm{NaI}$ solution $(2 \mathrm{~mL})$. The reaction mixture was refluxed, using an oil bath, for 30 mins then diluted with DCM $(10 \mathrm{~mL})$. The organic layer was washed with $\mathrm{H}_{2} \mathrm{O}(2 \times 40 \mathrm{~mL})$, dried over $\mathrm{Na}_{2} \mathrm{SO}_{4}$, filtered and the solvent was removed under reduced pressure to give a purple solid. The crude product was purified through silica gel column chromatography (DCM) to give 5,5-difluoro-3,7-diiodo-10-(3-nitrophenyl)- $5 H-4 \lambda^{4}, 5 \lambda^{4}$-dipyrrolo[1,2-c:2', $1^{\prime}$ $f][1,3,2]$ diazaborinine $(74.1 \mathrm{mg}, 0.13 \mathrm{mmol}, 98 \%)$ as a purple solid. 


\section{5,5-difluoro-3,7-diiodo-10-(3-nitrophenyl)-5H-4 $\lambda^{4}, 5 \lambda^{4}$-dipyrrolo[1,2-c:2',1'-}

\section{$f][1,3,2]$ diazaborinine $(3 \mathrm{~b})$}

Rf: 0.73 (DCM). Mp: $115-116{ }^{\circ} \mathrm{C} .{ }^{1} \mathbf{H}$ NMR (300 MHz, Chloroform- $d$ ) $\delta 8.44$ (ddd, $J=8.1$, 2.2, $\left.1.2 \mathrm{~Hz}, 1 \mathrm{H}, \mathrm{H}^{2}\right), 8.36\left(\mathrm{t}, J=1.8 \mathrm{~Hz}, 1 \mathrm{H}, \mathrm{H}^{5}\right), 7.83\left(\mathrm{dt}, \mathrm{J}=7.6,1.3 \mathrm{~Hz}, 1 \mathrm{H}, \mathrm{H}^{4}\right), 7.74(\mathrm{t}, J=$ $\left.7.9 \mathrm{~Hz}, 1 \mathrm{H}, \mathrm{H}^{3}\right), 6.74\left(\mathrm{~d}, J=4.3 \mathrm{~Hz}, 2 \mathrm{H}, \mathrm{H}^{9}\right), 6.59\left(\mathrm{~d}, J=4.2 \mathrm{~Hz}, 2 \mathrm{H}, \mathrm{H}^{10}\right) .{ }^{13} \mathbf{C} \mathbf{N M R}(75$ MHz, Chloroform- $d$ ) $\delta 148.3\left(\mathrm{C}^{1}\right), 137.7\left(\mathrm{C}^{8}\right), 137.4\left(\mathrm{C}^{5}\right), 135.9\left(\mathrm{C}^{4}\right), 134.2\left(\mathrm{C}^{7}\right), 131.3\left(\mathrm{C}^{10}\right)$, 131.0 - $130.8\left(\mathrm{~m}, \mathrm{C}^{9}\right), 130.1\left(\mathrm{C}^{3}\right), 125.5\left(\mathrm{C}^{2}\right), 124.9\left(\mathrm{C}^{6}\right), 105.1\left(\mathrm{C}^{11}\right) .{ }^{19} \mathbf{F}$ NMR $(282 \mathrm{MHz}$, Chloroform- $d$ ) $\delta$-144.72 (dq, $J=29.3,10.4$ Hz). ${ }^{11}$ B NMR $(96 \mathrm{MHz}$, Chloroform- $d$ ) $\delta 0.73$ (t, $J=29.0 \mathrm{~Hz}$ ). IR (neat): $\mathrm{v}_{\max } / \mathrm{cm}^{-1} 2920(\mathrm{C}-\mathrm{H}, \mathrm{w}), 1527$ (N-O, s). HRMS: (ASAP+) calcd for $\mathrm{C}_{15} \mathrm{H}_{8} \mathrm{BI}_{2} \mathrm{~F}_{2} \mathrm{~N}_{3} \mathrm{O}_{2}[\mathrm{M}+\mathrm{H}]^{+}: 565.8848$, found 565.8842. UV-Vis: $\lambda_{\max }=548 \mathrm{~nm}$ (DCM). Molar extinction coefficient $(\varepsilon)=73,000 \mathrm{M}^{-1} \mathrm{~cm}^{-1}$. $\phi_{\mathrm{F}}: 0.35$ 


\section{5,5-difluoro-3,7-diiodo-10-(4-methoxyphenyl)-5H-4 $\lambda^{4}, 5 \lambda^{4}$-dipyrrolo[1,2-c:2',1'- $f][1,3,2]$ diazaborinine $(3 \mathrm{c})$}

\section{Method A:}

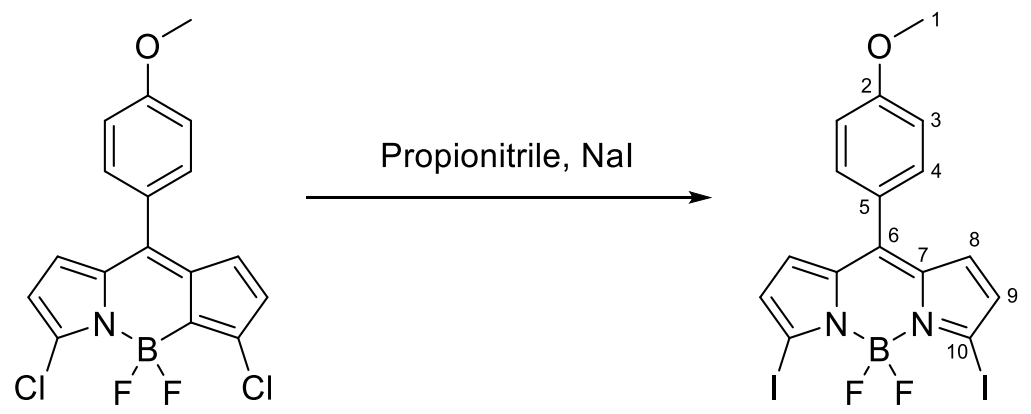

To a $25 \mathrm{~mL}$ round bottom flask was added methyl 3,7-dichloro-5,5-difluoro-10-(4methoxyphenyl)-5H-4 $\lambda^{4}, 5 \lambda^{4}$-dipyrrolo[1,2-c:2',1'-f][1,3,2]diazaborinine (34.5 mg, $0.09 \mathrm{mmol}$ ) and saturated NaI solution $(2 \mathrm{~mL})$. The reaction mixture was refluxed, using an oil bath, for 144 hours then diluted with DCM $(10 \mathrm{~mL})$. The organic layer was washed with $\mathrm{H}_{2} \mathrm{O}(2 \times 40$ $\mathrm{mL}$ ), dried over $\mathrm{Na}_{2} \mathrm{SO}_{4}$, filtered and the solvent was removed under reduced pressure to give a purple solid. The crude product was purified through silica gel column chromatography (3:1 Petrol:ethyl acetate) to give 5,5-difluoro-3,7-diiodo-10-(4-methoxyphenyl)- $5 H-4 \lambda^{4}, 5 \lambda^{4}$ dipyrrolo[1,2-c:2',1'-f][1,3,2]diazaborinine $(26.3 \mathrm{mg}, 0.05 \mathrm{mmol} 51 \%)$ as a purple solid.

\section{Method B:}

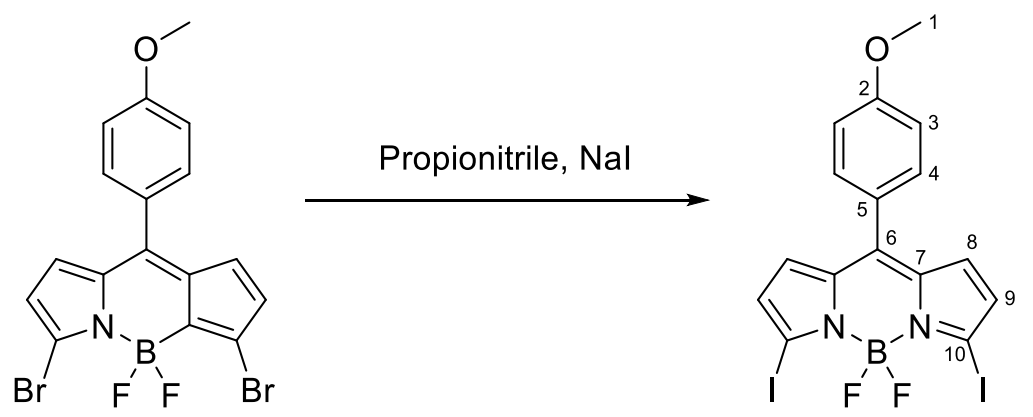

To a $25 \mathrm{~mL}$ round bottom flask was added methyl 3,7-dibromo-5,5-difluoro-10-(4methoxyphenyl)-5H-4 $\lambda^{4}, 5 \lambda^{4}$-dipyrrolo[1,2-c:2', $\left.1^{\prime}-f\right][1,3,2]$ diazaborinine $\quad(308.8 \mathrm{mg}, \quad 0.68$ $\mathrm{mmol})$ and saturated $\mathrm{NaI}$ solution $(6 \mathrm{~mL})$. The reaction mixture was refluxed, using an oil bath, for 2.5 hours then diluted with DCM $(50 \mathrm{~mL})$. The organic layer was washed with $\mathrm{H}_{2} \mathrm{O}(2 \mathrm{x}$ $100 \mathrm{~mL}$ ), dried over $\mathrm{Na}_{2} \mathrm{SO}_{4}$, filtered and the solvent was removed under reduced pressure to give a purple solid. The crude product was purified through silica gel column chromatography 
(DCM) to give 5,5-difluoro-3,7-diiodo-10-(4-methoxyphenyl)- $5 H-4 \lambda^{4}, 5 \lambda^{4}$-dipyrrolo[1,2c:2', $\left.1^{\prime}-f\right][1,3,2]$ diazaborinine $(231.7 \mathrm{mg}, 0.42 \mathrm{mmol}, 62 \%)$ as a purple solid.

\section{5,5-difluoro-3,7-diiodo-10-(4-methoxyphenyl)-5H-4 $\lambda^{4}, 5 \lambda^{4}$-dipyrrolo[1,2-c:2',1'-}

\section{$f][1,3,2]$ diazaborinine $(3 c)$}

Rf: 0.83 (DCM). Mp: $180-183{ }^{\circ} \mathrm{C} .{ }^{1} \mathbf{H}$ NMR $(300 \mathrm{MHz}$, Chloroform- $d$ ) $\delta 7.44(\mathrm{~d}, J=8.8 \mathrm{~Hz}$, $\left.2 \mathrm{H}, \mathrm{H}^{3}\right), 7.02\left(\mathrm{~d}, J=8.8 \mathrm{~Hz}, 2 \mathrm{H}, \mathrm{H}^{4}\right), 6.71-6.70\left(\mathrm{~m}, 4 \mathrm{H}, \mathrm{H}^{8,9}\right), 3.90\left(\mathrm{~s}, 3 \mathrm{H}, \mathrm{H}^{1}\right) .{ }^{13} \mathbf{C}$ NMR $(75$ MHz, Chloroform- $d$ ) $\delta 162.2\left(\mathrm{C}^{2}\right), 141.7\left(\mathrm{C}^{5}\right), 138.0\left(\mathrm{C}^{6}\right), 132.3\left(\mathrm{C}^{3}\right), 131.8\left(\mathrm{C}^{8}\right), 130.0-129.8$ $\left(\mathrm{m}, \mathrm{C}^{9}\right), 125.1\left(\mathrm{C}^{7}\right), 114.3\left(\mathrm{C}^{4}\right), 102.0\left(\mathrm{C}^{10}\right), 55.7\left(\mathrm{C}^{1}\right) .{ }^{19}$ F NMR $(282 \mathrm{MHz}$, Chloroform- $d) \delta$ $-144.47(\mathrm{~d}, J=29.7 \mathrm{~Hz}) .{ }^{11} \mathbf{B}$ NMR $(96 \mathrm{MHz}$, Chloroform- $d) \delta 0.69$ (t, $\left.J=29.3 \mathrm{~Hz}\right)$. IR (neat): Vmax $/ \mathrm{cm}^{-1} 2985$ (C-H, w). HRMS: (ASAP+) calcd for $\mathrm{C}_{16} \mathrm{H}_{11} \mathrm{BF}_{2} \mathrm{I}_{2} \mathrm{~N}_{2} \mathrm{O}[\mathrm{M}+\mathrm{H}]^{+}: 550.9103$, found 550.9101. UV-Vis: $\lambda_{\max }=537 \mathrm{~nm}$ (DCM). Molar extinction coefficient $(\varepsilon)=66000 \mathrm{M}^{-}$ ${ }^{1} \mathrm{~cm}^{-1} \cdot \phi_{\mathrm{F}}: 0.14$ 


\section{5,5-difluoro-3,7-diiodo-10-(3-methoxyphenyl)-5H-4 $\lambda^{4}, 5 \lambda^{4}$-dipyrrolo[1,2-c:2',1'- $f][1,3,2]$ diazaborinine $(3 \mathrm{~d})$}

\section{Method A:}

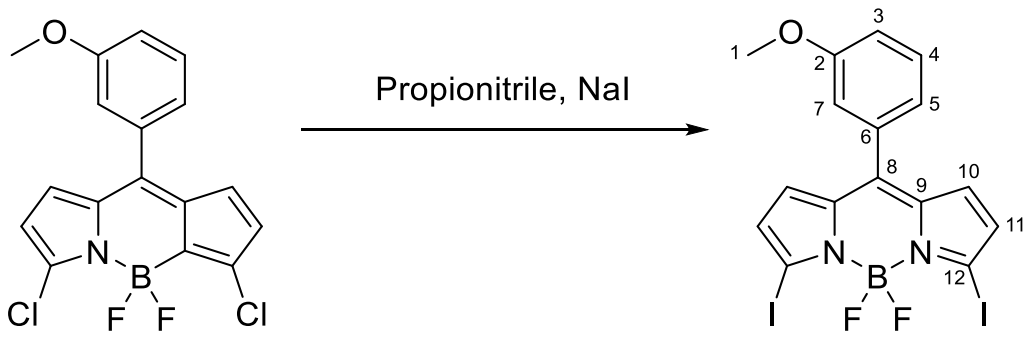

To a $25 \mathrm{~mL}$ round bottom flask was added methyl 3,7-dichloro-5,5-difluoro-10-(3methoxyphenyl)-5H-4 $\lambda^{4}, 5 \lambda^{4}$-dipyrrolo[1,2-c:2',1'-f][1,3,2]diazaborinine (38.5 mg, $0.11 \mathrm{mmol}$ ) and saturated $\mathrm{NaI}$ solution $(2 \mathrm{~mL})$. The reaction mixture was refluxed, using an oil bath, for 72 hours then diluted with DCM $(10 \mathrm{~mL})$. The organic layer was washed with $\mathrm{H}_{2} \mathrm{O}(2 \times 100 \mathrm{~mL})$, dried over $\mathrm{Na}_{2} \mathrm{SO}_{4}$, filtered and the solvent was removed under reduced pressure to give a purple solid. The crude product was purified through silica gel column chromatography (DCM) to give 5,5-difluoro-3,7-diiodo-10-(3-methoxyphenyl)- $5 H-4 \lambda^{4}, 5 \lambda^{4}$-dipyrrolo[1,2-c:2', $1^{\prime}$ $f][1,3,2]$ diazaborinine $(52.6 \mathrm{mg}, 0.096 \mathrm{mmol}, 91 \%)$ as a purple solid.

\section{Method B:}

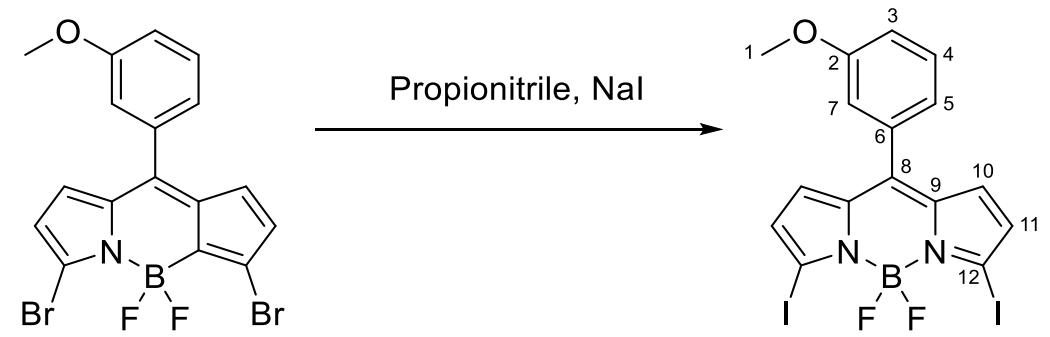

To a $25 \mathrm{~mL}$ round bottom flask was added methyl 3,7-dibromo-5,5-difluoro-10-(3methoxyphenyl)-5H-4 $\lambda^{4}, 5 \lambda^{4}$-dipyrrolo[1,2-c:2',1'-f][1,3,2]diazaborinine $\quad(342.4 \mathrm{mg}, \quad 0.75$ mmol) and saturated $\mathrm{NaI}$ solution $(6 \mathrm{~mL})$. The reaction mixture was refluxed, using an oil bath, for 2.5 hours then diluted with DCM $(50 \mathrm{~mL})$. The organic layer was washed with $\mathrm{H}_{2} \mathrm{O}(2 \mathrm{x}$ $100 \mathrm{~mL}$ ), dried over $\mathrm{Na}_{2} \mathrm{SO}_{4}$, filtered and the solvent was removed under reduced pressure to give a purple solid. The crude product was purified through silica gel column chromatography (DCM) to give 5,5-difluoro-3,7-diiodo-10-(3-methoxyphenyl)- $5 H-4 \lambda^{4}, 5 \lambda^{4}$-dipyrrolo[1,2c:2',1'-f][1,3,2]diazaborinine (278 $\mathrm{mg}, 0.51 \mathrm{mmol}, 88 \%)$ as a purple solid. 


\section{5,5-difluoro-3,7-diiodo-10-(3-methoxyphenyl)-5H-4 $\lambda^{4}, 5 \lambda^{4}$-dipyrrolo[1,2-c:2',1'-}

\section{$f][1,3,2]$ diazaborinine $(3 \mathrm{~d})$}

Rf: 0.80 (DCM). Mp: 210-211 C. ${ }^{1}$ H NMR (300 MHz, Chloroform- $d$ ) $\delta 7.40(\mathrm{dd}, J=8.1,7.5$ $\left.\mathrm{Hz}, 1 \mathrm{H}, \mathrm{H}^{4}\right), 7.11\left(\mathrm{ddd}, J=8.4,2.6,1.0 \mathrm{~Hz}, 1 \mathrm{H}, \mathrm{H}^{5}\right), 7.06\left(\mathrm{ddd}, J=7.5,1.6,1.0 \mathrm{~Hz}, 1 \mathrm{H}, \mathrm{H}^{3}\right)$, $7.02\left(\mathrm{dd}, J=2.6,1.6 \mathrm{~Hz}, 1 \mathrm{H}, \mathrm{H}^{7}\right), 6.73-6.68\left(\mathrm{~m}, 4 \mathrm{H}, \mathrm{H}^{10,11}\right), 3.85\left(\mathrm{~s}, 3 \mathrm{H}, \mathrm{H}^{1}\right) .{ }^{13} \mathrm{C}$ NMR $(75$ MHz, Chloroform- $d) \delta 159.5\left(\mathrm{C}^{2}\right), 141.2\left(\mathrm{C}^{6}\right), 138.0\left(\mathrm{C}^{9}\right), 133.9\left(\mathrm{C}^{8}\right), 131.9\left(\mathrm{C}^{10}\right), 130.2$ $130.0\left(\mathrm{~m}, \mathrm{C}^{11}\right), 129.8\left(\mathrm{C}^{4}\right), 122.9\left(\mathrm{C}^{3}\right), 116.3\left(\mathrm{C}^{5}\right), 116.1\left(\mathrm{C}^{7}\right), 103.1\left(\mathrm{C}^{12}\right), 55.6\left(\mathrm{C}^{1}\right) .{ }^{19} \mathbf{F}$ NMR (282 MHz, Chloroform- $d$ ) $\delta-144.69$ (q, $J=29.4 \mathrm{MHz}) .{ }^{11} \mathbf{B}$ NMR (96 MHz, Chloroform- $d$ ) $\delta$ $0.71(\mathrm{t}, J=30.2 \mathrm{~Hz})$. IR (neat): $\mathrm{v}_{\max } / \mathrm{cm}^{-1} 3156$ (C-H, w) 2958-2902 (C-H, w). HRMS: (ASAP+) calcd for $\mathrm{C}_{16} \mathrm{H}_{11} \mathrm{BF}_{2} \mathrm{I}_{2} \mathrm{~N}_{2} \mathrm{O}[\mathrm{M}+\mathrm{H}]^{+}: 550.9103$, found 550.9102. UV-Vis: $\lambda_{\max }=541$ $\mathrm{nm}(\mathrm{DCM})$. Molar extinction coefficient $(\varepsilon)=76000 \mathrm{M}^{-1} \mathrm{~cm}^{-1} \cdot \phi_{\mathrm{F}}: 0.12$. 
Methyl 4-(5,5-difluoro-3,7-diphenyl-5H-4 $\lambda^{4}, 5 \lambda^{4}$-dipyrrolo[1,2-c:2',1'-

$f][1,3,2]$ diazaborinin-10-yl)benzoate (4) and methyl 4-(3-butyl-5,5-difluoro-7-phenyl-

$5 H-5 \lambda 4,6 \lambda 4-d i p y r r o l o\left[1,2-c: 2^{\prime}, 1^{\prime}-f\right][1,3,2]$ diazaborinin-10-yl)benzoate (5)

\section{Method A:}

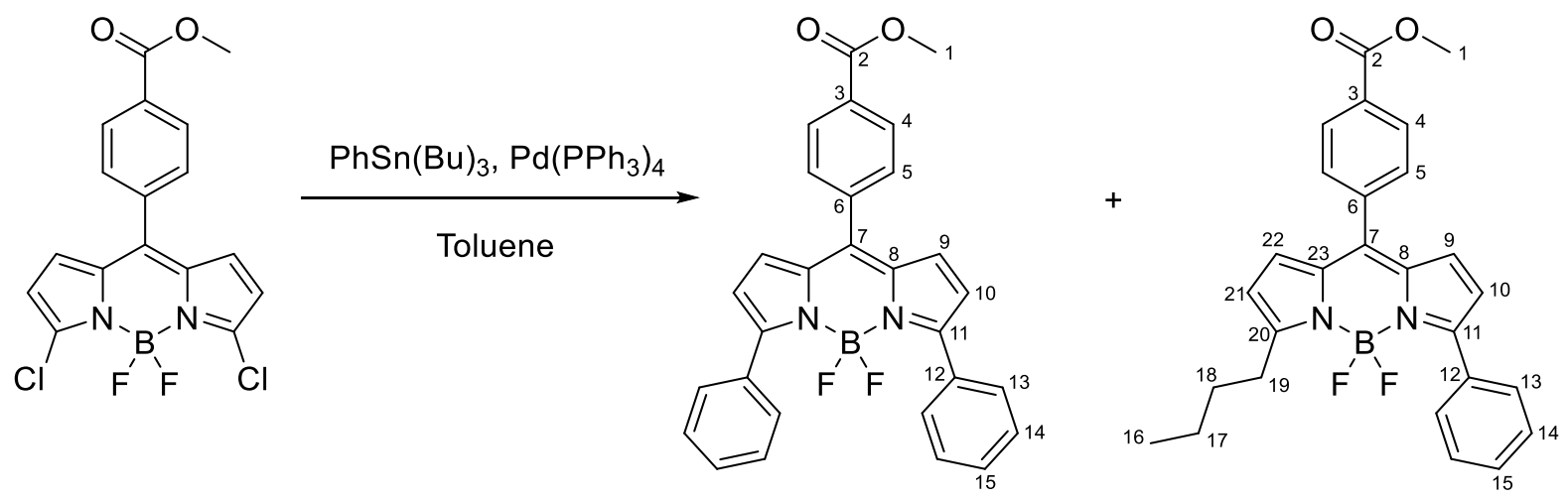

To a $50 \mathrm{~mL}$ Schlenk flask, under nitrogen atmosphere, was added methyl 4-(3,7-dichloro-5,5difluoro-5 $H-4 \lambda^{4}, 5 \lambda^{4}$-dipyrrolo[1,2-c:2',1'-f][1,3,2]diazaborinin-10-yl)benzoate (39.5 mg, 0.1 $\mathrm{mmol}), \mathrm{PhSn}(\mathrm{Bu})_{3}(73.4 \mathrm{mg}, 0.2 \mathrm{mmol}), \mathrm{Pd}\left(\mathrm{PPh}_{3}\right)_{4}(11 \mathrm{mg}, 10 \mathrm{~mol} \%, 0.01 \mathrm{mmol})$, dry toluene $(6 \mathrm{~mL})$ and was degassed using freeze thaw method $(\mathrm{x} 3)$. The reaction mixture was heated, using an oil bath, to $100^{\circ} \mathrm{C}$, stirred for 16 hours then left to cool to room temp (10 mins). To the crude reaction mixture was added water $(10 \mathrm{~mL})$ and the product was extracted with DCM $\left(3 \times 30 \mathrm{~mL}\right.$ ). The organic layer was dried over $\mathrm{MgSO}_{4}$, filtered and the solvent was removed under reduced pressure to give a dark purple solid. The crude product was purified by silica gel column chromatography (1:1 petrol:DCM) to give methyl 4-(5,5-difluoro-3,7-diphenyl$5 \mathrm{H}-4 \lambda^{4}, 5 \lambda^{4}$-dipyrrolo[1,2-c:2',1'-f][1,3,2]diazaborinin-10-yl)benzoate (16.7 mg, $0.035 \mathrm{mmol}$,

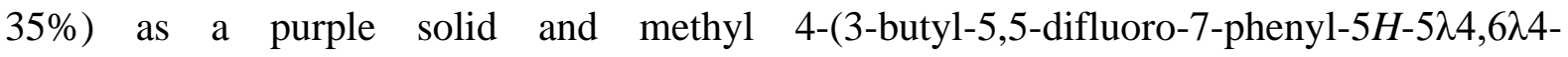
dipyrrolo[1,2-c:2',1'-f][1,3,2] diazaborinin-10-yl)benzoate (10.4 $\mathrm{mg}, 0.024 \mathrm{mmol}, 24 \%)$ as a purple solid. 


\section{Method B:}

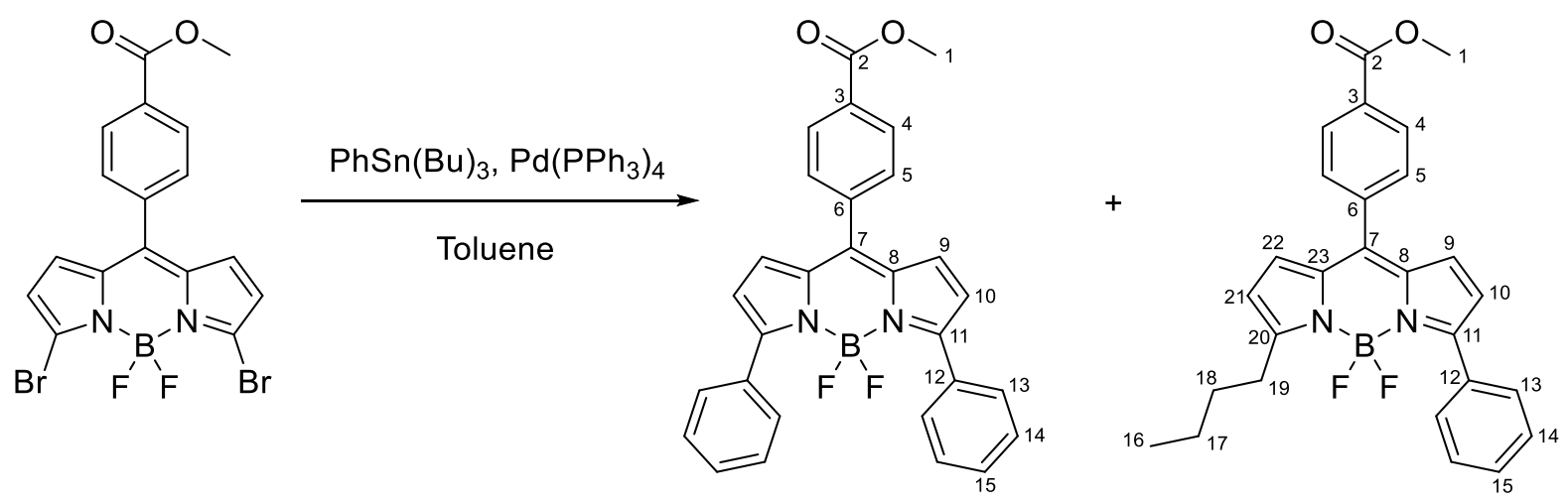

To a $50 \mathrm{~mL}$ Schlenk flask, under nitrogen atmosphere, was added methyl 4-(3,7-dibromo-5,5difluoro-5H-4 $\lambda^{4}, 5 \lambda^{4}$-dipyrrolo[1,2-c:2', $\left.1^{\prime}-f\right][1,3,2]$ diazaborinin-10-yl)benzoate (48.2 mg, 0.1 mmol), $\mathrm{PhSn}(\mathrm{Bu})_{3}(73.4 \mathrm{mg}, 0.2 \mathrm{mmol}), \mathrm{Pd}\left(\mathrm{PPh}_{3}\right)_{4}(11 \mathrm{mg}, 10 \mathrm{~mol} \%, 0.01 \mathrm{mmol})$, dry toluene $(6 \mathrm{~mL})$ and was degassed using freeze thaw method $(\mathrm{x} 3)$. The reaction mixture was heated, using an oil bath, to $100^{\circ} \mathrm{C}$, stirred for 16 hours then left to cool to room temp (10 mins). To the crude reaction mixture was added water $(10 \mathrm{~mL})$ and the product was extracted with DCM ( $3 \times 30 \mathrm{~mL}$ ). The organic layer was dried over $\mathrm{MgSO}_{4}$, filtered and the solvent was removed under reduced pressure to give a dark purple solid. The crude product was purified by silica gel column chromatography (1:1 petrol:DCM) to give methyl 4-(5,5-difluoro-3,7-diphenyl$5 \mathrm{H}-4 \lambda^{4}, 5 \lambda^{4}$-dipyrrolo[1,2-c:2',1'-f][1,3,2]diazaborinin-10-yl)benzoate (36.6 mg, $0.077 \mathrm{mmol}$,

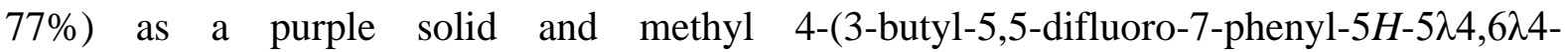
dipyrrolo[1,2-c:2',1'-f][1,3,2]diazaborinin-10-yl)benzoate (4.0 $\mathrm{mg}, 0.008 \mathrm{mmol}, 8 \%)$ as a purple solid.

\section{Method C:}

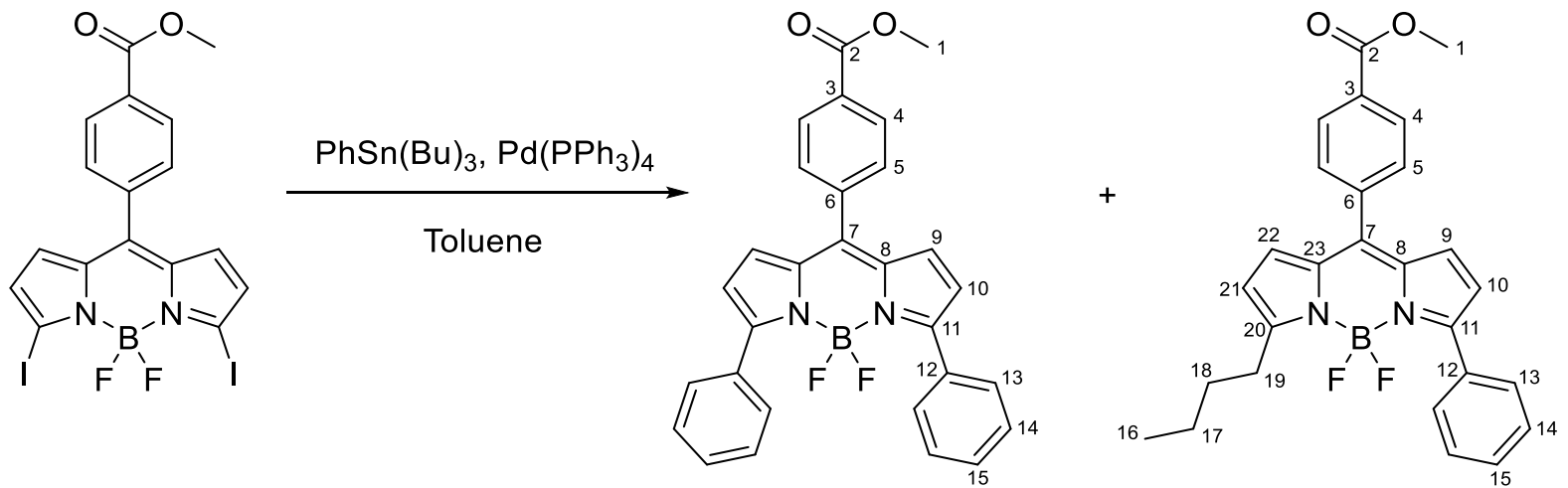

To a $50 \mathrm{~mL}$ Schlenk flask, under nitrogen atmosphere, was added methyl 4-(3,7-diiodo-5,5difluoro-5H-4 $\lambda^{4}, 5 \lambda^{4}$-dipyrrolo[1,2-c:2',1'-f][1,3,2]diazaborinin-10-yl)benzoate (57.8 mg, 0.1 mmol), $\mathrm{PhSn}(\mathrm{Bu})_{3}(73.4 \mathrm{mg}, 0.2 \mathrm{mmol}), \mathrm{Pd}\left(\mathrm{PPh}_{3}\right)_{4}(11 \mathrm{mg}, 10 \mathrm{~mol} \%, 0.01 \mathrm{mmol})$, dry toluene 
$(6 \mathrm{~mL})$ and was degassed using freeze thaw method (x3). The reaction mixture was heated, using an oil bath, to $100^{\circ} \mathrm{C}$, stirred for 16 hours then left to cool to room temp (10 mins). To the crude reaction mixture was added water $(10 \mathrm{~mL})$ and the product was extracted with DCM ( 3 x $30 \mathrm{~mL}$ ). The organic layer was dried over $\mathrm{MgSO}_{4}$, filtered and the solvent was removed under reduced pressure to give a dark purple solid. The crude product was purified by silica gel column chromatography (1:1 petrol:DCM) to give methyl 4-(5,5-difluoro-3,7-diphenyl$5 \mathrm{H}-4 \lambda^{4}, 5 \lambda^{4}$-dipyrrolo[1,2-c:2',1'-f][1,3,2]diazaborinin-10-yl)benzoate $(24.2 \mathrm{mg}, 0.051 \mathrm{mmol}$,

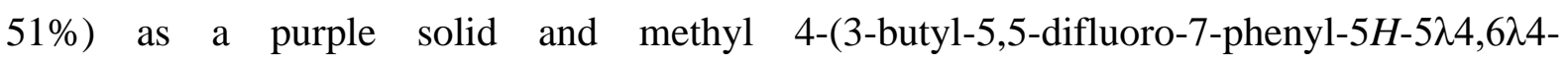
dipyrrolo[1,2-c:2',1'-f][1,3,2]diazaborinin-10-yl)benzoate $(8.8 \mathrm{mg}, 0.017 \mathrm{mmol}, 17 \%)$ as a purple solid.

\section{Methyl}

4-(5,5-difluoro-3,7-diphenyl-5H-4 $\lambda^{4}, 5 \lambda^{4}$-dipyrrolo[1,2-c:2',1'-

\section{$f][1,3,2]$ diazaborinin-10-yl)benzoate (4)}

Rf: 0.18 (1:1 40/60 petroleum ether : DCM). Mp: 104-105 ${ }^{\circ} \mathrm{C} .{ }^{1} \mathbf{H}$ NMR $(300 \mathrm{MHz}$, Chloroform- $d$ ) $\delta 8.22\left(\mathrm{~d}, J=8.5 \mathrm{~Hz}, 2 \mathrm{H}, \mathrm{H}^{4}\right), 7.99-7.81\left(\mathrm{~m}, 4 \mathrm{H}, \mathrm{H}^{13}\right), 7.68(\mathrm{~d}, J=8.5 \mathrm{~Hz}$, $\left.2 \mathrm{H}, \mathrm{H}^{5}\right), 7.54-7.37\left(\mathrm{~m}, 6 \mathrm{H}, \mathrm{H}^{14,15}\right), 6.84\left(\mathrm{~d}, J=4.3 \mathrm{~Hz}, 2 \mathrm{H}, \mathrm{H}^{9}\right), 6.64\left(\mathrm{~d}, J=4.1 \mathrm{~Hz}, 2 \mathrm{H}, \mathrm{H}^{10}\right)$, $4.00\left(\mathrm{~s}, 3 \mathrm{H}, \mathrm{H}^{1}\right) .{ }^{13} \mathrm{C}$ NMR (75 MHz, Chloroform- $\left.d\right) \delta 166.5\left(\mathrm{C}^{2}\right), 159.6\left(\mathrm{C}^{11}\right), 142.5\left(\mathrm{C}^{7}\right)$, $138.9\left(\mathrm{C}^{3}\right), 136.2\left(\mathrm{C}^{8}\right), 132.6\left(\mathrm{C}^{12}\right), 131.8\left(\mathrm{C}^{6}\right), 130.7\left(\mathrm{C}^{5,9}\right), 129.8\left(\mathrm{C}^{15}\right), 129.6\left(\mathrm{C}^{13}\right), 129.6$ $\left(\mathrm{C}^{4}\right), 128.4\left(\mathrm{C}^{14}\right), 121.4\left(\mathrm{C}^{10}\right), 52.6\left(\mathrm{C}^{1}\right) .{ }^{19} \mathrm{~F}$ NMR $(282 \mathrm{MHz}$, Chloroform- $d$ ) $\delta-132.47(\mathrm{q}, J$ $=31.8 \mathrm{~Hz}) .{ }^{11} \mathbf{B}$ NMR $(96 \mathrm{MHz}$, Chloroform- $d) \delta 1.43(\mathrm{t}, J=31.2 \mathrm{~Hz})$. IR (neat): $\mathrm{vmax} / \mathrm{cm}^{-1}$ 2973 (C-H, m), 1876 (C=O, w). HRMS: (ASAP+) calcd for $\mathrm{C}_{29} \mathrm{H}_{21} \mathrm{BF}_{2} \mathrm{~N}_{2} \mathrm{O}_{2}[\mathrm{M}+\mathrm{H}]^{+}$: 479.1747, found 479.1745. UV-Vis: $\lambda_{\max }=557 \mathrm{~nm}(\mathrm{DCM})$. Molar extinction coefficient $(\varepsilon)=$ $58000 \mathrm{M}^{-1} \mathrm{~cm}^{-1}$. $\mathbf{F}: 0.03$.

\section{Methyl}

4-(3-butyl-5,5-difluoro-7-phenyl-5H-5 $\lambda 4,6 \lambda 4$-dipyrrolo[1,2-c:2',1'$f[[1,3,2]$ diazaborinin-10-yl)benzoate (5)

Rf: 0.38 (1:1 40/60 petroleum ether : DCM). Mp: 169-170 ${ }^{\circ} \mathrm{C} .{ }^{1} \mathbf{H}$ NMR $(300 \mathrm{MHz}$, Chloroform- $d$ ) $\delta 8.18\left(\mathrm{~d}, J=8.1 \mathrm{~Hz}, 2 \mathrm{H}, \mathrm{H}^{4}\right), 7.95-7.86\left(\mathrm{~m}, 2 \mathrm{H}, \mathrm{H}^{13}\right), 7.63(\mathrm{~d}, J=8.1 \mathrm{~Hz}$, $\left.2 \mathrm{H}, \mathrm{H}^{5}\right), 7.57-7.38\left(\mathrm{~m}, 3 \mathrm{H}, \mathrm{H}^{14,15}\right), 6.76\left(\mathrm{~d}, J=4.3 \mathrm{~Hz}, 1 \mathrm{H}, \mathrm{H}^{22}\right), 6.74\left(\mathrm{~d}, J=4.1 \mathrm{~Hz}, 1 \mathrm{H}, \mathrm{H}^{9}\right)$, $6.59\left(\mathrm{~d}, J=4.1 \mathrm{~Hz}, 1 \mathrm{H}, \mathrm{H}^{10}\right), 6.41\left(\mathrm{~d}, J=4.3 \mathrm{~Hz}, 1 \mathrm{H}, \mathrm{H}^{21}\right), 3.99\left(\mathrm{~s}, 3 \mathrm{H}, \mathrm{H}^{1}\right), 3.03(\mathrm{t}, J=7.8$ $\left.\mathrm{Hz}, 2 \mathrm{H}, \mathrm{H}^{19}\right), 1.71\left(\mathrm{p}, J=7.7 \mathrm{~Hz}, 2 \mathrm{H}, \mathrm{H}^{18}\right), 1.43\left(\mathrm{~h}, J=7.4 \mathrm{~Hz}, 2 \mathrm{H}, \mathrm{H}^{17}\right), 0.94$ (t, $J=7.3 \mathrm{~Hz}$, $\left.3 \mathrm{H}, \mathrm{H}^{16}\right) .{ }^{13} \mathrm{C}$ NMR (176 MHz, Chloroform-d) $\delta 166.6\left(\mathrm{C}^{2}\right), 165.5\left(\mathrm{C}^{20}\right), 157.7\left(\mathrm{C}^{11}\right), 141.7$ $\left(C^{7}\right), 138.8\left(C^{3}\right), 135.7\left(C^{8}\right), 134.6\left(C^{23}\right), 132.9\left(C^{12}\right), 131.7\left(C^{6}\right), 131.2\left(C^{22}\right), 130.6\left(C^{5}\right), 129.7$ 
$\left(\mathrm{C}^{9}\right), 129.6\left(\mathrm{C}^{4}\right), 129.5\left(\mathrm{C}^{15}\right), 129.5\left(\mathrm{C}^{13}\right), 128.4\left(\mathrm{C}^{14}\right), 120.1\left(\mathrm{C}^{10}\right), 119.7\left(\mathrm{C}^{21}\right), 52.6\left(\mathrm{C}^{1}\right), 30.7$ $\left(\mathrm{C}^{18}\right), 28.9\left(\mathrm{C}^{19}\right), 22.8\left(\mathrm{C}^{17}\right), 14.1\left(\mathrm{C}^{16}\right) .{ }^{19}$ F NMR $(282 \mathrm{MHz}$, Chloroform- $d$ ) $\delta-138.27(\mathrm{q}, J=$ $32.1 \mathrm{~Hz}) .{ }^{11} \mathbf{B}$ NMR $(96 \mathrm{MHz}$, Chloroform- $d) \delta 1.21$ (t, $\left.J=33.3 \mathrm{~Hz}\right) . \mathbf{I R}$ (neat): $\mathrm{V}_{\max } / \mathrm{cm}^{-1} 3001$ (C-H, s), 2994 (C-H, m), 1710 (C=O, m). HRMS: (ASAP+) calcd for $\mathrm{C}_{27} \mathrm{H}_{25} \mathrm{BF}_{2} \mathrm{~N}_{2} \mathrm{O}_{2}[\mathrm{M}+\mathrm{H}]^{+}$: 459.2050, found 459.2052. UV-Vis: $\lambda_{\max }=538 \mathrm{~nm}(\mathrm{DCM})$. Molar extinction coefficient $(\varepsilon)=$ $67000 \mathrm{M}^{-1} \mathrm{~cm}^{-1} \cdot \phi_{\mathrm{F}}: 0.04$ 


\section{Method A:}

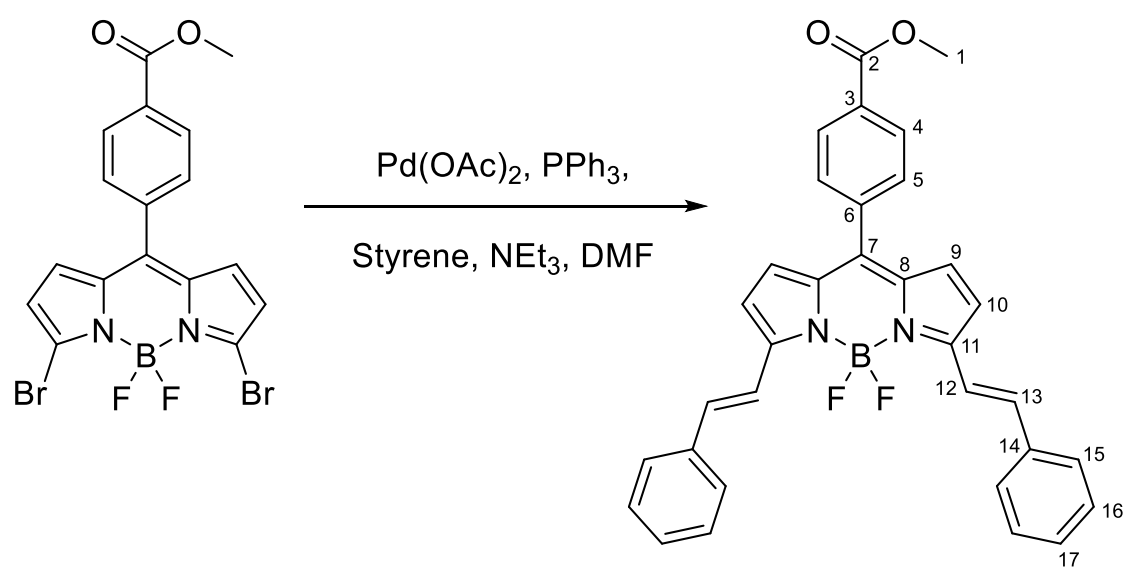

To a $50 \mathrm{~mL}$ Schlenck flask, under nitrogen atmosphere, was added methyl 4-(3,7-dibromo-5,5difluoro-5H-4 $\lambda^{4}, 5 \lambda^{4}$-dipyrrolo[1,2-c:2',1'-f][1,3,2]diazaborinin-10-yl)benzoate (48.2 mg, 0.1 $\mathrm{mmol}), \mathrm{Pd}(\mathrm{OAc})_{2}(0.7 \mathrm{mg}, 0.003 \mathrm{mmol}, 3 \mathrm{~mol} \%), \mathrm{PPh}_{3}(2 \mathrm{mg}, 0.007 \mathrm{mmol}, 7 \mathrm{~mol} \%)$ and was stirred for 10 mins. To the reaction mixture was added styrene $(28.7 \mu \mathrm{L}, 0.25 \mathrm{mmol}), \mathrm{NEt}_{3}$ (41.8 $\mu \mathrm{L}, 0.3 \mathrm{mmol})$, dry DMF (0.5 mL), degassed (x3) and heated, using an oil bath, to $100^{\circ} \mathrm{C}$. The reaction mixture was stirred for 8 hours, quenched with water $(20 \mathrm{~mL})$ and the organic layer was extracted with DCM (3 x $20 \mathrm{~mL}$ ). The organic layer was dried over $\mathrm{MgSO}_{4}$, filtered and the solvent was removed under reduced pressure to give a purple solid. The crude product was purified by silica gel column chromatography (1:2 petrol:DCM) to give methyl 4-(5,5difluoro-3,7-di((E)-styryl)-5H-4 $\lambda^{4}, 5 \lambda^{4}$-dipyrrolo[1,2-c:2',1'-f][1,3,2]diazaborinin-10yl)benzoate (1.4 mg, $0.003 \mathrm{mmol}, 3 \%)$ as a blue solid. 


\section{Method B:}

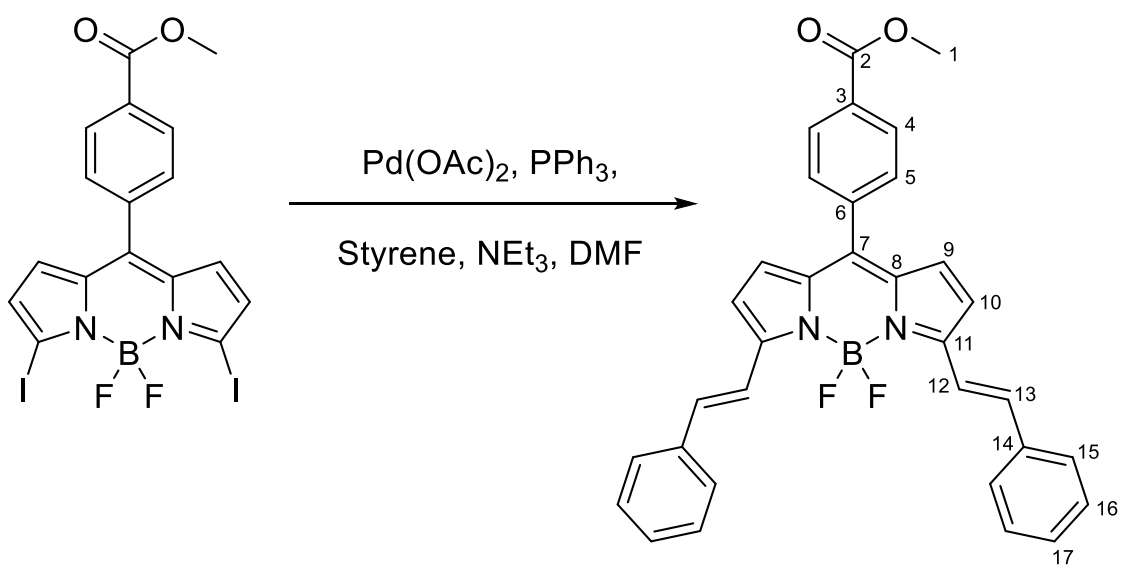

To a 50 mL Schlenck flask, under nitrogen atmosphere, was added methyl 4-(3,7-diiodo-5,5difluoro-5H-4 $4 \lambda^{4}, 5 \lambda^{4}$-dipyrrolo[1,2-c:2',1'-f][1,3,2]diazaborinin-10-yl)benzoate (57.8 mg, 0.1 $\mathrm{mmol}), \mathrm{Pd}(\mathrm{OAc})_{2}(0.7 \mathrm{mg}, 0.003 \mathrm{mmol}, 3 \mathrm{~mol} \%), \mathrm{PPh}_{3}(2 \mathrm{mg}, 0.007 \mathrm{mmol}, 7 \mathrm{~mol} \%)$ and was stirred for 10 mins. To the reaction mixture was added styrene $(28.7 \mu \mathrm{L}, 0.25 \mathrm{mmol})$, NEt3 $(41.8 \mu \mathrm{L}, 0.3 \mathrm{mmol})$, dry DMF (0.5 mL), degassed (x3) and heated, using an oil bath, to $100^{\circ} \mathrm{C}$. The reaction mixture was stirred for 8 hours, quenched with water $(20 \mathrm{~mL})$ and the organic layer was extracted with DCM (3 x $20 \mathrm{~mL})$. The organic layer was dried over $\mathrm{MgSO}_{4}$, filtered and the solvent was removed under reduced pressure to give a purple solid. The crude product was purified by silica gel column chromatography $(1: 2$ petrol:DCM) to give methyl 4-(5,5difluoro-3,7-di((E)-styryl)-5H-4 $\lambda^{4}, 5 \lambda^{4}$-dipyrrolo[1,2-c:2',1'-f][1,3,2]diazaborinin-10yl)benzoate (16 mg, $0.030 \mathrm{mmol}, 30 \%$ ) as a blue solid.

\section{Methyl 4-(5,5-difluoro-3,7-di((E)-styryl)-5H-4 $\lambda^{4}, 5 \lambda^{4}$-dipyrrolo[1,2-c:2',1'- $f][1,3,2]$ diazaborinin-10-yl)benzoate (6)}

Rf: 0.40 (1:2 40/60 petroleum ether : DCM). Mp: 263-264 ${ }^{\circ} \mathrm{C} .{ }^{1} \mathbf{H}$ NMR $(300 \mathrm{MHz}$, Chloroform- $d$ ) $\delta 8.17\left(\mathrm{~d}, J=8.5 \mathrm{~Hz}, 2 \mathrm{H}, \mathrm{H}^{4}\right), 7.80\left(\mathrm{~d}, J=16.4 \mathrm{~Hz}, 2 \mathrm{H}, \mathrm{H}^{12}\right), 7.67(\mathrm{~d}, J=7.1$ $\left.\mathrm{Hz}, 4 \mathrm{H}, \mathrm{H}^{15}\right), 7.60\left(\mathrm{~d}, J=8.5 \mathrm{~Hz}, 2 \mathrm{H}, \mathrm{H}^{5}\right), 7.48-7.36\left(\mathrm{~m}, 6 \mathrm{H}, \mathrm{H}^{16,17}\right), 7.36(\mathrm{~d}, J=16.4 \mathrm{~Hz}$, $\left.2 \mathrm{H}, \mathrm{H}^{13}\right), 6.95\left(\mathrm{~d}, J=4.5 \mathrm{~Hz}, 2 \mathrm{H}, \mathrm{H}^{10}\right), 6.76\left(\mathrm{~d}, J=4.5 \mathrm{~Hz}, 2 \mathrm{H}, \mathrm{H}^{9}\right), 3.99\left(\mathrm{~s}, 3 \mathrm{H}, \mathrm{H}^{1}\right) .{ }^{13} \mathbf{C ~ N M R}$ (75 MHz, Chloroform- $d$ ) $\delta 166.6\left(\mathrm{C}^{2}\right), 155.4\left(\mathrm{C}^{11}\right), 138.9\left(\mathrm{C}^{3}\right), 137.9\left(\mathrm{C}^{7}\right), 137.3\left(\mathrm{C}^{13}\right), 136.5$ $\left(\mathrm{C}^{14}\right), 136.1\left(\mathrm{C}^{8}\right), 131.5\left(\mathrm{C}^{6}\right), 130.6\left(\mathrm{C}^{5}\right), 129.6\left(\mathrm{C}^{4}\right), 129.5\left(\mathrm{C}^{9}\right), 129.4\left(\mathrm{C}^{17}\right), 129.0\left(\mathrm{C}^{16}\right), 127.8$ $\left(\mathrm{C}^{15}\right), 119.4\left(\mathrm{C}^{12}\right), 116.8\left(\mathrm{C}^{10}\right), 52.6\left(\mathrm{C}^{1}\right) .{ }^{19} \mathbf{F}$ NMR $(282 \mathrm{MHz}$, Chloroform- $d$ ) $\delta-139.54(\mathrm{q}, J$ $=32.9 \mathrm{~Hz}) .{ }^{11} \mathbf{B}$ NMR $(96 \mathrm{MHz}$, Chloroform- $d) \delta 1.32$ (t, $\left.J=32.9 \mathrm{~Hz}\right)$. IR (neat): $\mathrm{V}_{\max } / \mathrm{cm}^{-1}$ $2921(\mathrm{C}-\mathrm{H}, \mathrm{s}) 2852(\mathrm{C}-\mathrm{H}, \mathrm{m}) 1722(\mathrm{C}=\mathrm{O}, \mathrm{m})$. HRMS: (ASAP+) calcd for $\mathrm{C}_{33} \mathrm{H}_{25} \mathrm{BF}_{2} \mathrm{~N}_{2} \mathrm{O}_{2}$ 
$[\mathrm{M}+\mathrm{H}]^{+}:$531.2061, found 531.2063. UV-Vis: $\lambda_{\max }=619 \mathrm{~nm}(\mathrm{DCM})$. Molar extinction coefficient $(\varepsilon)=96000 \mathrm{M}^{-1} \mathrm{~cm}^{-1} . \phi_{\mathrm{F}}: 0.03$ 


\section{Method A:}<smiles></smiles>
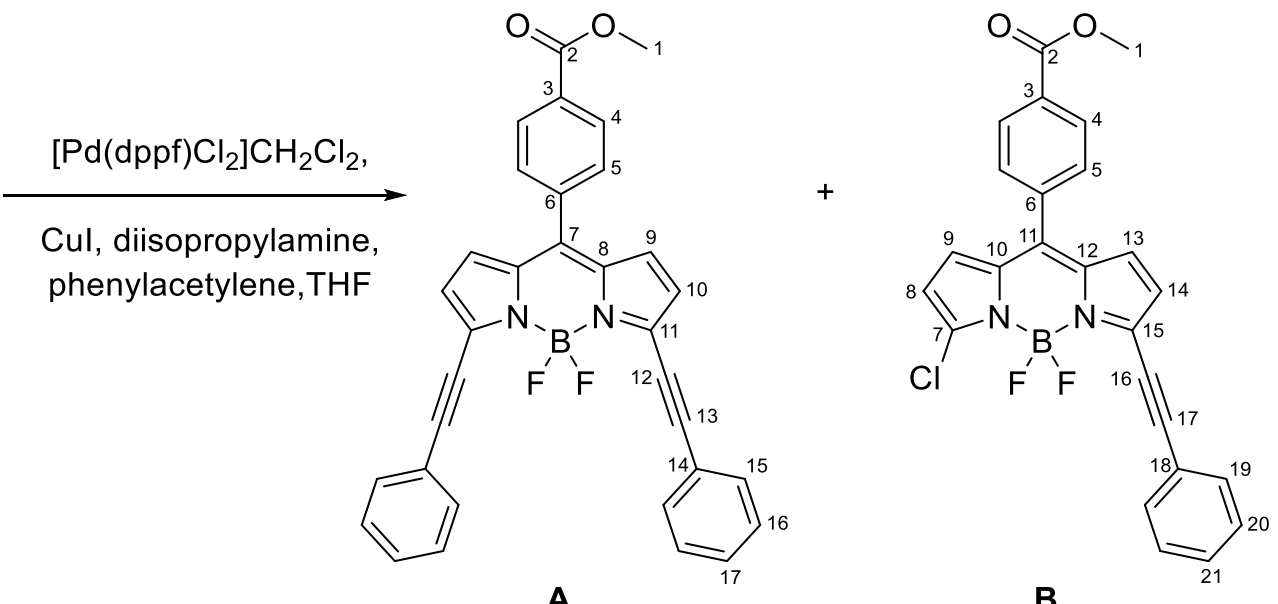

A

B

To a round bottom flask was added methyl 4-(3,7-dichloro-5,5-difluoro-5H-4 $\lambda^{4}, 5 \lambda^{4}$ dipyrrolo[1,2-c:2',1'-f][1,3,2]diazaborinin-10-yl)benzoate $\quad(48.2 \quad \mathrm{mg}, \quad 0.1 \quad \mathrm{mmol})$, $\left[\mathrm{Pd}(\mathrm{dppf}) \mathrm{Cl}_{2}\right] \mathrm{CH}_{2} \mathrm{Cl}_{2}(4.1 \mathrm{mg}, 0.005 \mathrm{mmol}, 5 \mathrm{~mol} \%), \mathrm{CuI}(1.0 \mathrm{mg}, 0.005 \mathrm{mmol}, 5 \mathrm{~mol} \%)$, dry THF (3 mL), diisopropylamine (1.9 mL, $11.0 \mathrm{mmol})$ and phenylacetylene ( $89 \mu \mathrm{L}, 0.81 \mathrm{mmol})$. The resulting reaction mixture was stirred at room temp for 48 hours and subsequently poured into cold distilled water $(20 \mathrm{~mL})$ then extracted with DCM $(3 \times 10 \mathrm{~mL})$. The combined extracted reaction mixture was washed with water $(3 \times 15 \mathrm{~mL})$, dried over $\mathrm{MgSO}_{4}$, filtered and the solvent was removed under reduced pressure to give a dark purple solid. The crude product was purified through silica gel column chromatography (1:1 petrol: diethyl ether) to give methyl

4-(5,5-difluoro-3,7-bis(phenylethynyl)-5H-4 $\lambda^{4}, 5 \lambda^{4}$-dipyrrolo[1,2-c:2',1'$f][1,3,2]$ diazaborinin-10-yl)benzoate $(5.3 \mathrm{mg}, 0.01 \mathrm{mmol}, 10 \%)$ as a blue solid and methyl 4(3-chloro-5,5-difluoro-7-(phenylethynyl)-5H-4 $\lambda^{4}, 5 \lambda^{4}$-dipyrrolo[1,2-c:2',1'$f][1,3,2]$ diazaborinin-10-yl)benzoate $(6.8 \mathrm{mg}, 0.015 \mathrm{mmol}, 15 \%)$ as a pink solid. 


\section{Method B:}

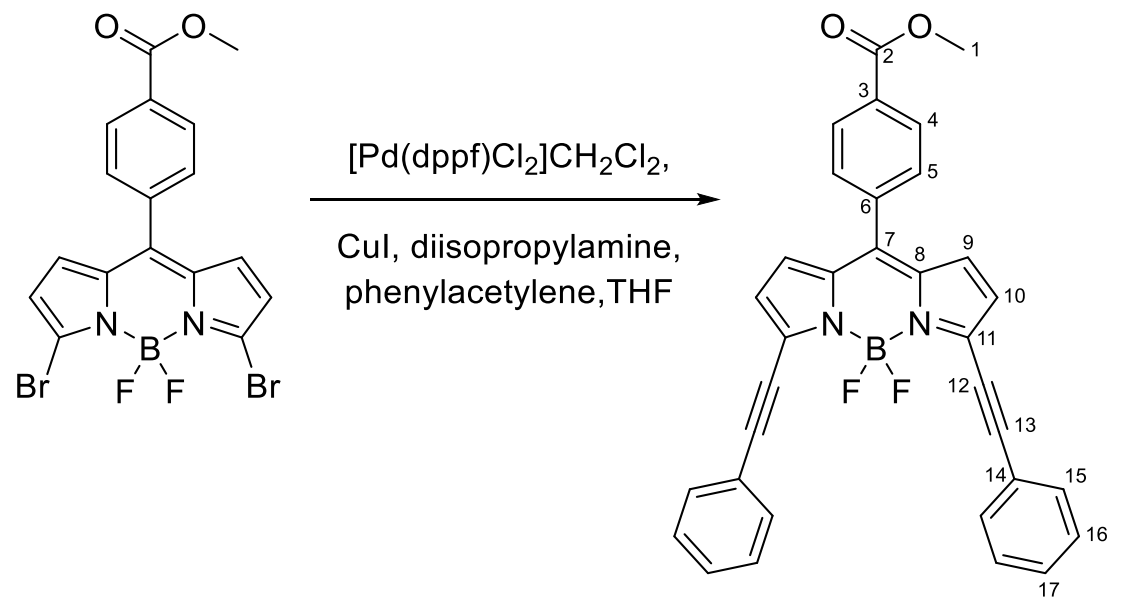

To a round bottom flask was added methyl 4-(3,7-dibromo-5,5-difluoro- $5 H-4 \lambda^{4}, 5 \lambda^{4}$ dipyrrolo[1,2-c:2',1'-f][1,3,2]diazaborinin-10-yl)benzoate $\quad(48.2 \quad \mathrm{mg}, \quad 0.1 \quad \mathrm{mmol})$, $\left[\mathrm{Pd}(\mathrm{dppf}) \mathrm{Cl}_{2}\right] \mathrm{CH}_{2} \mathrm{Cl}_{2}$ (4.1 mg, $\left.0.005 \mathrm{mmol}, 5 \mathrm{~mol} \%\right)$, CuI (1.0 mg, $\left.0.005 \mathrm{mmol}, 5 \mathrm{~mol} \%\right)$, dry THF (3 mL), diisopropylamine (1.9 mL, $11.0 \mathrm{mmol})$ and phenylacetylene $(89 \mu \mathrm{L}, 0.81 \mathrm{mmol})$. The resulting reaction mixture was stirred at room temp for 48 hours and subsequently poured into cold distilled water $(20 \mathrm{~mL})$ then extracted with DCM $(3 \times 10 \mathrm{~mL})$. The combined extracted reaction mixture was washed with water $(3 \times 15 \mathrm{~mL})$, dried over $\mathrm{MgSO}_{4}$, filtered and the solvent was removed under reduced pressure to give a dark purple solid. The crude product was purified through silica gel column chromatography (1:1 petrol: diethyl ether) to give methyl

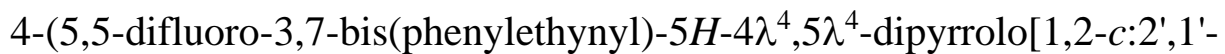
$f][1,3,2]$ diazaborinin-10-yl)benzoate (25.4 $\mathrm{mg}, 0.049 \mathrm{mmol}, 49 \%)$ as a blue solid. 


\section{Method C:}

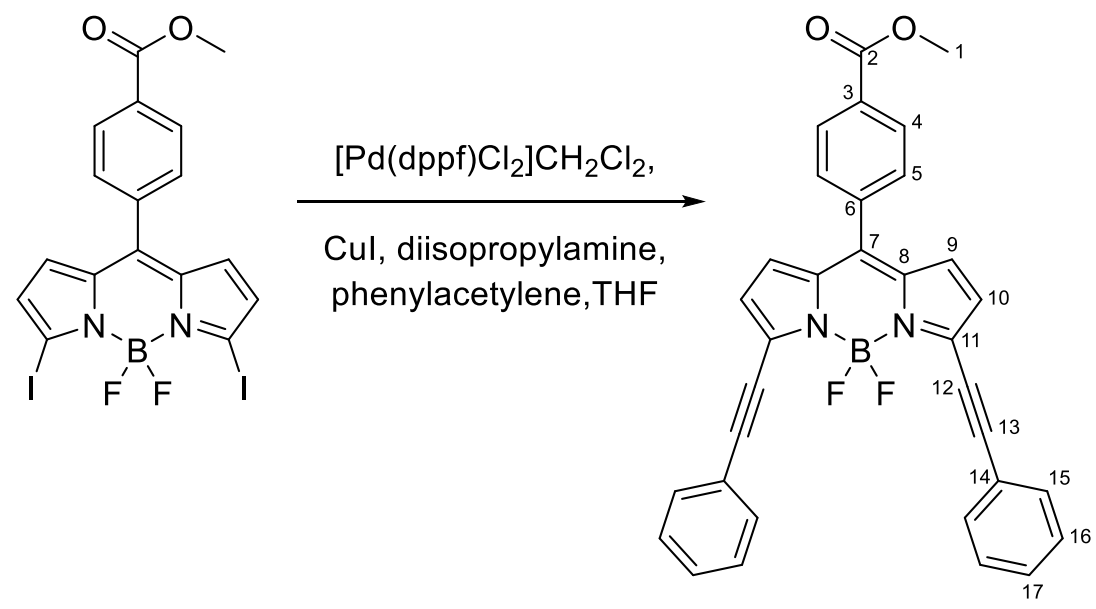

To a round bottom flask was added methyl 4-(3,7-diiodo-5,5-difluoro- $5 H-4 \lambda^{4}, 5 \lambda^{4}$ dipyrrolo[1,2-c:2',1'-f][1,3,2]diazaborinin-10-yl)benzoate $\quad(57.8 \quad \mathrm{mg}, \quad 0.1 \quad \mathrm{mmol})$, $\left[\mathrm{Pd}(\mathrm{dppf}) \mathrm{Cl}_{2}\right] \mathrm{CH}_{2} \mathrm{Cl}_{2}$ (4.1 mg, $\left.0.005 \mathrm{mmol}, 5 \mathrm{~mol} \%\right)$, CuI (1.0 mg, $\left.0.005 \mathrm{mmol}, 5 \mathrm{~mol} \%\right)$, dry THF (3 mL), diisopropylamine (1.9 mL, $11.0 \mathrm{mmol})$ and phenylacetylene ( $89 \mu \mathrm{L}, 0.81 \mathrm{mmol})$. The resulting reaction mixture was stirred at room temp for 48 hours and subsequently poured into cold distilled water $(20 \mathrm{~mL})$ then $\mathrm{CC}$, extracted with DCM (3 x $10 \mathrm{~mL})$. The combined extracted reaction mixture was washed with water $(3 \times 15 \mathrm{~mL})$, dried over $\mathrm{MgSO}_{4}$, filtered and the solvent was removed under reduced pressure to give a dark purple solid. The crude product was purified through silica gel column chromatography (1:1 petrol: diethyl ether) to give methyl 4-(5,5-difluoro-3,7-bis(phenylethynyl)-5H-4 $\lambda^{4}, 5 \lambda^{4}$-dipyrrolo[1,2-c:2',1'$f][1,3,2]$ diazaborinin-10-yl)benzoate $(32.1 \mathrm{mg}, 0.061 \mathrm{mmol}, 61 \%)$ as a blue solid.

\section{Methyl}

4-(5,5-difluoro-3,7-bis(phenylethynyl)-5H-4 $\lambda^{4}, 5 \lambda^{4}$-dipyrrolo[1,2-c:2',1'$f[[1,3,2]$ diazaborinin-10-yl)benzoate (7)

Rf: 0.91 (DCM). Mp: 204-205 ${ }^{\circ}$ C. ${ }^{1} \mathbf{H}$ NMR $(300 \mathrm{MHz}$, Chloroform- $d$ ) $\delta 8.19(\mathrm{~d}, J=8.5 \mathrm{~Hz}$, $\left.2 \mathrm{H}, \mathrm{H}^{4}\right), 7.77-7.67\left(\mathrm{~m}, 4 \mathrm{H}, \mathrm{H}^{15}\right), 7.62\left(\mathrm{~d}, J=8.5 \mathrm{~Hz}, 2 \mathrm{H}, \mathrm{H}^{5}\right), 7.48-7.35\left(\mathrm{~m}, 6 \mathrm{H}, \mathrm{H}^{16,17}\right)$, $6.81\left(\mathrm{~d}, J=4.1 \mathrm{~Hz}, 2 \mathrm{H}, \mathrm{H}^{9}\right), 6.73\left(\mathrm{~d}, J=4.3 \mathrm{~Hz}, 2 \mathrm{H}, \mathrm{H}^{10}\right), 3.98\left(\mathrm{~s}, 3 \mathrm{H}, \mathrm{H}^{1}\right) .{ }^{13} \mathbf{C}$ NMR (75 MHz, Chloroform- $d$ ) $\delta 166.4\left(\mathrm{C}^{2}\right), 140.3\left(\mathrm{C}^{7}\right), 138.7\left(\mathrm{C}^{11}\right), 138.3\left(\mathrm{C}^{3}\right), 136.5\left(\mathrm{C}^{8}\right), 132.6\left(\mathrm{C}^{15}\right)$, $132.0\left(\mathrm{C}^{6}\right), 130.7\left(\mathrm{C}^{5}\right), 130.2\left(\mathrm{C}^{9}\right), 129.8\left(\mathrm{C}^{17}\right), 129.7\left(\mathrm{C}^{4}\right), 128.6\left(\mathrm{C}^{16}\right), 124.2\left(\mathrm{C}^{10}\right), 122.4\left(\mathrm{C}^{18}\right)$, $103.6\left(\mathrm{C}^{13}\right), 83.5\left(\mathrm{C}^{12}\right), 52.6\left(\mathrm{C}^{1}\right) .{ }^{19} \mathbf{F}$ NMR $(282 \mathrm{MHz}$, Chloroform- $d) \delta-146.86(\mathrm{q}, J=27.6$ Hz). ${ }^{11}$ B NMR (96 MHz, Chloroform- $d$ ) $\delta 0.73$ (t, $\left.J=27.9 \mathrm{~Hz}\right)$. IR (neat): $\mathrm{v}_{\max } / \mathrm{cm}^{-1} 2937$ (C$\mathrm{H}, \mathrm{s}), 2190(\mathrm{C} \equiv \mathrm{C}, \mathrm{m}), 1718(\mathrm{C}=\mathrm{O}, \mathrm{m})$. HRMS: $(\mathrm{ASAP}+)$ calcd for $\mathrm{C}_{33} \mathrm{H}_{21} \mathrm{BF}_{2} \mathrm{~N}_{2} \mathrm{O}_{2}[\mathrm{M}+\mathrm{H}]^{+}$: 
527.1748, found 527.1751. UV-Vis: $\lambda_{\max }=619 \mathrm{~nm}(\mathrm{DCM})$. Molar extinction coefficient $(\varepsilon)=$ $85000 \mathrm{M}^{-1} \mathrm{~cm}^{-1} \cdot \phi_{\mathrm{F}}: 0.78$.

Methyl 4-(3-chloro-5,5-difluoro-7-(phenylethynyl)-5H-4 $\lambda^{4}, 5 \lambda^{4}$-dipyrrolo[1,2-c:2',1'$f][1,3,2]$ diazaborinin-10-yl)benzoate (8)

Rf: 0.74 (DCM). Mp: 216-217 ${ }^{\circ}$ C. ${ }^{1} \mathbf{H}$ NMR (400 MHz, Chloroform- $d$ ) $\delta 8.19$ (d, $J=8.5 \mathrm{~Hz}$, $\left.2 \mathrm{H}, \mathrm{H}^{4}\right), 7.70-7.66\left(\mathrm{~m}, 2 \mathrm{H}, \mathrm{H}^{19}\right), 7.59\left(\mathrm{~d}, J=8.5 \mathrm{~Hz}, 2 \mathrm{H}, \mathrm{H}^{5}\right), 7.43-7.37\left(\mathrm{~m}, 3 \mathrm{H}, \mathrm{H}^{20,21}\right)$, $6.81\left(\mathrm{~d}, J=4.4 \mathrm{~Hz}, 1 \mathrm{H}, \mathrm{H}^{13}\right), 6.79\left(\mathrm{~d}, J=4.3 \mathrm{~Hz}, 1 \mathrm{H}, \mathrm{H}^{9}\right), 6.73\left(\mathrm{~d}, J=4.4 \mathrm{~Hz}, 1 \mathrm{H}, \mathrm{H}^{14}\right), 6.45$ $\left(\mathrm{d}, J=4.4 \mathrm{~Hz}, 1 \mathrm{H}, \mathrm{H}^{8}\right), 3.99\left(\mathrm{~s}, 3 \mathrm{H}, \mathrm{H}^{l}\right) .{ }^{13} \mathbf{C}$ NMR $(176 \mathrm{MHz}$, Chloroform- $d) \delta 166.3\left(\mathrm{C}^{2}\right)$, 145.6 $\left(\mathrm{C}^{7}\right), 141.4\left(\mathrm{C}^{11}\right), 139.0\left(\mathrm{C}^{15}\right), 137.5\left(\mathrm{C}^{3}\right), 135.7\left(\mathrm{C}^{12}\right), 134.4\left(\mathrm{C}^{10}\right), 132.6\left(\mathrm{C}^{19}\right), 132.2$ $\left(\mathrm{C}^{6}\right), 131.0\left(\mathrm{C}^{9}\right), 130.7\left(\mathrm{C}^{13}\right), 130.6\left(\mathrm{C}^{5}\right), 130.0\left(\mathrm{C}^{21}\right), 129.8\left(\mathrm{C}^{4}\right), 128.6\left(\mathrm{C}^{20}\right), 124.4\left(\mathrm{C}^{14}\right), 122.0$ $\left(\mathrm{C}^{18}\right), 119.2\left(\mathrm{C}^{8}\right), 103.5\left(\mathrm{C}^{17}\right), 82.8\left(\mathrm{C}^{16}\right), 52.7\left(\mathrm{C}^{1}\right) .{ }^{19} \mathbf{F}$ NMR $(282 \mathrm{MHz}$, Chloroform- $d) \delta$ $147.53(\mathrm{~d}, J=27.6 \mathrm{~Hz}) .{ }^{11} \mathbf{B}$ NMR $(96 \mathrm{MHz}$, Chloroform- $d$ ) $\delta 0.59(\mathrm{t}, J=28.0 \mathrm{~Hz}) . \mathbf{I R}$ (neat): $\mathrm{V}_{\max } / \mathrm{cm}^{-1} 3054(\mathrm{C}-\mathrm{H}, \mathrm{w}), 1717(\mathrm{C}=\mathrm{O}, \mathrm{m})$. HRMS: (ASAP+) calcd for $\mathrm{C}_{25} \mathrm{H}_{16} \mathrm{BClF}_{2} \mathrm{~N}_{2} \mathrm{O}_{2}$ $[\mathrm{M}+\mathrm{H}]^{+}:$461.1044, found 461.1037. UV-Vis: $\lambda_{\max }=567 \mathrm{~nm}(\mathrm{DCM})$. Molar extinction coefficient $(\varepsilon)=60000 \mathrm{M}^{-1} \mathrm{~cm}^{-1} \cdot \phi_{\mathrm{F}}: 0.60$. 


\section{Method A:}

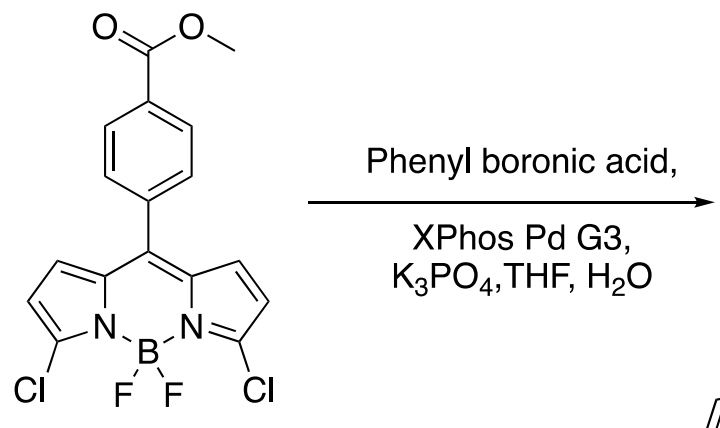

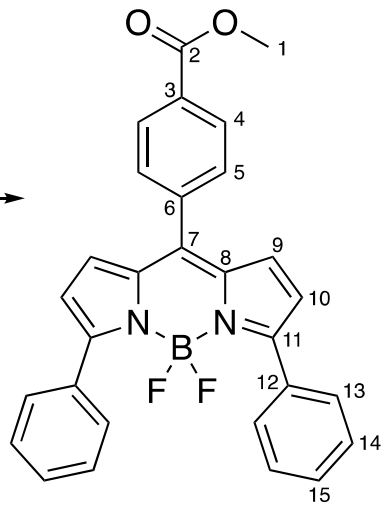

A

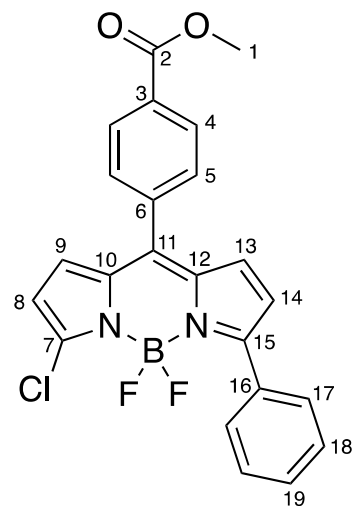

B

To a $50 \mathrm{~mL}$ Schlenk flask, under nitrogen atmosphere, was added methyl 4-(3,7-dichloro-5,5difluoro-5H-4 $\lambda^{4}, 5 \lambda^{4}$-dipyrrolo[1,2-c:2', $\left.1^{\prime}-f\right][1,3,2]$ diazaborinin-10-yl)benzoate $(39.5 \mathrm{mg}, 0.1$ mmol), phenyl boronic acid (48.8 mg, $0.4 \mathrm{mmol})$, XPhos Pd G3 (6.8 mg, $0.008 \mathrm{mmol}, 8 \mathrm{~mol} \%$ ), $\mathrm{K}_{3} \mathrm{PO}_{4}(92.1 \mathrm{mg}, 0.4 \mathrm{mmol})$, dry THF (8 mL), water $(1.2 \mathrm{~mL})$ and degassed $(\mathrm{x} 3)$. The reaction mixture was heated, using an oil bath, to $75^{\circ} \mathrm{C}$, stirred for 16 hours then diluted with DCM (50 $\mathrm{mL})$. The reaction mixture was washed with water $(3 \times 50 \mathrm{~mL})$, the organic layer was dried over $\mathrm{MgSO}_{4}$, filtered and the solvent was removed under reduced pressure to give a purple solid. The crude product was purified by silica gel column chromatography (DCM) to give methyl 4-(5,5-difluoro-3,7-diphenyl-5H-4 $\lambda^{4}, 5 \lambda^{4}$-dipyrrolo[1,2-c:2',1'-f][1,3,2]diazaborinin10-yl)benzoate $(3.1 \mathrm{mg}, 0.006 \mathrm{mmol}, 6 \%)$ as a purple solid and methyl 4-(3-chloro-5,5difluoro-7-phenyl-5H-5 $\lambda^{4}, 6 \lambda^{4}$-dipyrrolo[1,2-c:2',1'-f][1,3,2]diazaborinin-10-yl)benzoate (1.7 $\mathrm{mg}, 0.004 \mathrm{mmol}, 4 \%$ ) as a pink solid. 


\section{Method B:}

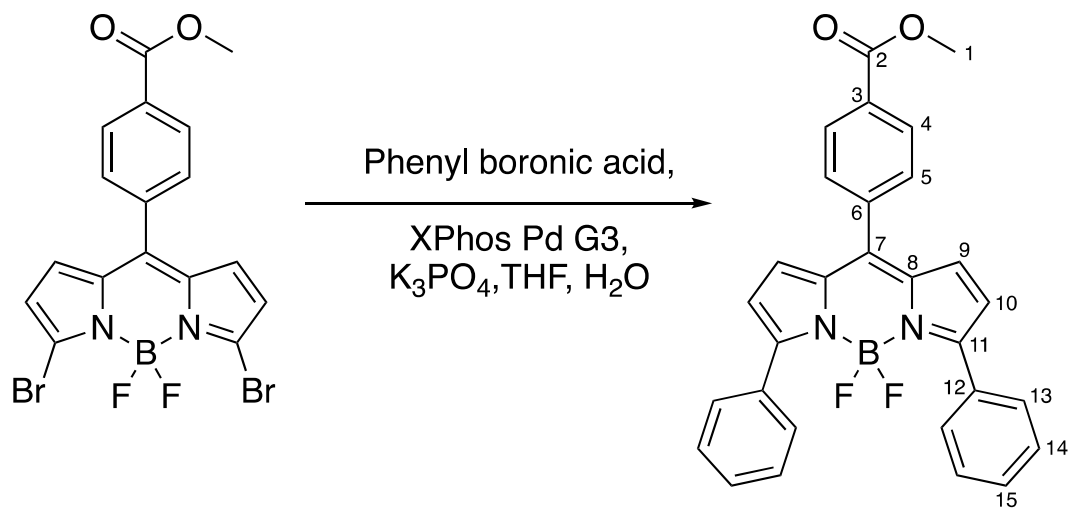

To a $50 \mathrm{~mL}$ Schlenk flask, under nitrogen atmosphere, was added methyl 4-(3,7-dibromo-5,5difluoro-5H-4 $4 \lambda^{4}, 5 \lambda^{4}$-dipyrrolo[1,2-c:2',1'-f][1,3,2]diazaborinin-10-yl)benzoate (48.2 mg, 0.1 mmol), phenyl boronic acid (48.8 mg, $0.4 \mathrm{mmol})$, XPhos Pd G3 (6.8 mg, $0.008 \mathrm{mmol}, 8 \mathrm{~mol} \%$ ), $\mathrm{K}_{3} \mathrm{PO}_{4}(92.1 \mathrm{mg}, 0.4 \mathrm{mmol})$, dry THF $(8 \mathrm{~mL})$, water $(1.2 \mathrm{~mL})$ and degassed (x3). The reaction mixture was stirred at room temp for 17 hours, diluted with DCM $(50 \mathrm{~mL})$ and washed with water $(3 \times 50 \mathrm{~mL})$. The organic layer was dried over $\mathrm{MgSO}_{4}$, filtered and the solvent was removed under reduced pressure to give a purple solid. The crude product was purified by silica gel column chromatography (DCM) to give methyl 4-(5,5-difluoro-3,7-diphenyl-5H-4 $\lambda^{4}, 5 \lambda^{4}$ dipyrrolo[1,2-c:2',1'-f][1,3,2]diazaborinin-10-yl)benzoate (38.7 $\mathrm{mg}, 0.081 \mathrm{mmol}, 81 \%)$ as a purple solid.

\section{Method C:}

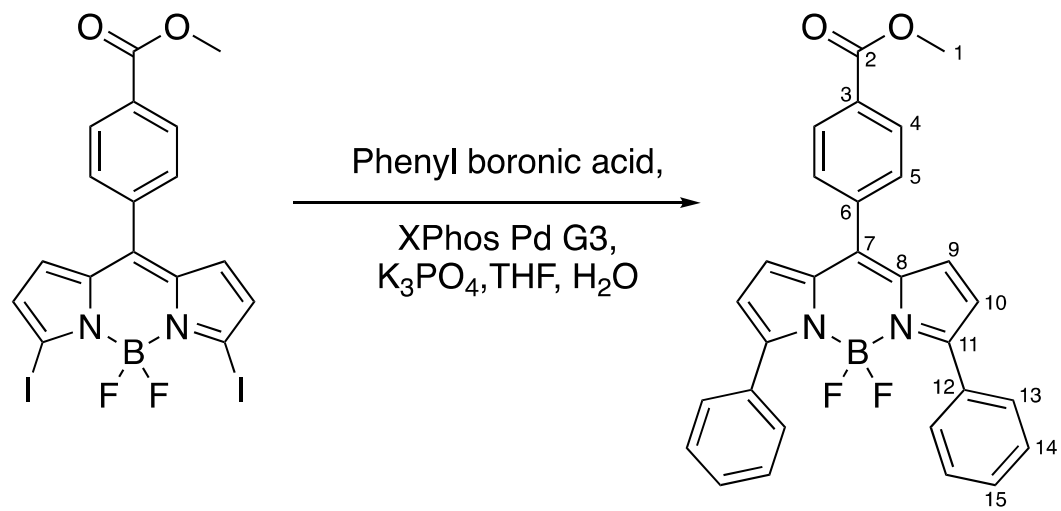

To a $50 \mathrm{~mL}$ Schlenk flask, under nitrogen atmosphere, was added methyl 4-(3,7-diiodo-5,5difluoro-5H-4 $\lambda^{4}, 5 \lambda^{4}$-dipyrrolo[1,2-c:2', $\left.1^{\prime}-f\right][1,3,2]$ diazaborinin-10-yl)benzoate (57.8 mg, 0.1 mmol), phenyl boronic acid (48.8 mg, $0.4 \mathrm{mmol}$ ), XPhos Pd G3 (6.8 mg, $0.008 \mathrm{mmol}, 8 \mathrm{~mol} \%$ ), $\mathrm{K}_{3} \mathrm{PO}_{4}(92.1 \mathrm{mg}, 0.4 \mathrm{mmol})$, dry THF $(8 \mathrm{~mL})$, water $(1.2 \mathrm{~mL})$ and degassed $(\mathrm{x} 3)$. The reaction mixture was stirred at room temp for 17 hours, diluted with DCM $(50 \mathrm{~mL})$ and washed with 
water $(3 \times 50 \mathrm{~mL})$. The organic layer was dried over $\mathrm{MgSO}_{4}$, filtered and the solvent was removed under reduced pressure to give a purple solid. The crude product was purified by silica gel column chromatography (DCM) to give methyl 4-(5,5-difluoro-3,7-diphenyl-5H-4 $\lambda^{4}, 5 \lambda^{4}$ dipyrrolo[1,2-c:2',1'-f][1,3,2]diazaborinin-10-yl)benzoate $(41.8 \mathrm{mg}, 0.087 \mathrm{mmol}, 87 \%)$ as a purple solid.

\section{Methyl}

4-(5,5-difluoro-3,7-diphenyl-5H-4 $\lambda^{4}, 5 \lambda^{4}$-dipyrrolo[1,2-c:2', $1^{\prime}-$

$f][1,3,2]$ diazaborinin-10-yl)benzoate (4)

Analytical data as reported above.

Methyl

4-(3-chloro-5,5-difluoro-7-phenyl-5H-5 $\lambda^{4}, 6 \lambda^{4}$-dipyrrolo[1,2-c:2',1'-

\section{$f][1,3,2]$ diazaborinin-10-yl)benzoate (9)}

Rf: 0.78 (DCM). Mp: $169-170{ }^{\circ}$ C. ${ }^{1} \mathbf{H}$ NMR $(300 \mathrm{MHz}$, Chloroform- $d$ ) $\delta 8.20(\mathrm{~d}, J=8.3 \mathrm{~Hz}$, $\left.2 \mathrm{H}, \mathrm{H}^{4}\right), 7.99-7.89\left(\mathrm{~m}, 2 \mathrm{H}, \mathrm{H}^{17}\right), 7.62\left(\mathrm{~d}, J=8.3 \mathrm{~Hz}, 2 \mathrm{H}, \mathrm{H}^{5}\right), 7.55-7.45\left(\mathrm{~m}, 3 \mathrm{H}, \mathrm{H}^{18,19}\right)$, $6.88\left(\mathrm{~d}, J=4.3 \mathrm{~Hz}, 1 \mathrm{H}, \mathrm{H}^{13}\right), 6.76-6.69\left(\mathrm{~m}, 2 \mathrm{H}, \mathrm{H}^{9,14}\right), 6.40\left(\mathrm{~d}, J=4.2 \mathrm{~Hz}, 1 \mathrm{H}, \mathrm{H}^{8}\right), 3.99$ (s, $\left.3 \mathrm{H}, \mathrm{H}^{1}\right) .{ }^{13} \mathrm{C}$ NMR $(75 \mathrm{MHz}$, Chloroform- $d) \delta 166.4\left(\mathrm{C}^{2}\right), 161.7\left(\mathrm{C}^{15}\right), 143.2\left(\mathrm{C}^{7}\right), 142.3\left(\mathrm{C}^{11}\right)$, $137.8\left(\mathrm{C}^{3}\right), 136.8\left(\mathrm{C}^{12}\right), 133.1\left(\mathrm{C}^{10}\right), 132.4\left(\mathrm{C}^{13}\right), 132.1\left(\mathrm{C}^{6}\right), 132.0\left(\mathrm{C}^{16}\right), 130.6\left(\mathrm{C}^{5}\right), 130.4$ $\left(\mathrm{C}^{19}\right), 129.7\left(\mathrm{C}^{4}\right), 129.6\left(\mathrm{C}^{17}\right), 129.6\left(\mathrm{C}^{9}\right), 128.6\left(\mathrm{C}^{18}\right), 122.2\left(\mathrm{C}^{14}\right), 118.5\left(\mathrm{C}^{8}\right), 52.7\left(\mathrm{C}^{1}\right) .{ }^{19} \mathrm{~F}$ NMR (282 MHz, Chloroform- $d$ ) $\delta-140.06$ (q, $J=29.8 \mathrm{~Hz}) .{ }^{11}$ B NMR (96 MHz, Chloroformd) $\delta 0.96$ (t, $J=30.2 \mathrm{~Hz}$ ). IR (neat): $\mathrm{V}_{\max } / \mathrm{cm}^{-1}$ 3055, 2997 (C-H, w), 1724 (C=O, m). HRMS: (ASAP+) calcd for $\mathrm{C}_{23} \mathrm{H}_{16} \mathrm{BClF}_{2} \mathrm{~N}_{2} \mathrm{O}_{2}[\mathrm{M}+\mathrm{H}]^{+}:$: 437.1044 , found 437.1036 . UV-Vis: $\lambda_{\max }=537$ $\mathrm{nm}(\mathrm{DCM})$. Molar extinction coefficient $(\varepsilon)=67000 \mathrm{M}^{-1} \mathrm{~cm}^{-1} \phi_{\mathrm{F}}: 0.03$. 


\section{Solubility Measurements for $\mathrm{NaCl}, \mathrm{NaBr}$ and $\mathrm{NaI}$ in Propionitrile}

Saturated solutions of $\mathrm{NaCl}, \mathrm{NaBr}$ and $\mathrm{NaI}$ in propionitrile were prepared at room temp. Each solution was stirred overnight to ensure equilibrium had been reached. The stirrer was switched off and solution allowed to stand for one hour, then a fixed amount of the supernatant was removed from each solution $(10 \mathrm{~mL})$, evaporated to dryness and the mass of the residue measured to determine the saturation limit.

$\mathrm{NaCl}$ in propionitrile $=140 \mathrm{mg} / \mathrm{L}$ or $2.42 \mathrm{mmol} / \mathrm{L}$

$\mathrm{NaBr}$ in propionitrile $=340 \mathrm{mg} / \mathrm{L}$ or $3.30 \mathrm{mmol} / \mathrm{L}$

$\mathrm{NaI}$ in propionitrile $=14,297 \mathrm{mg} / \mathrm{L}$ or $953.80 \mathrm{mmol} / \mathrm{L}$ 
UV/Vis absorption spectra and extinction coefficient measurements

Methyl 4-(5,5-difluoro-3,7-diiodo-5H-4 $\lambda^{4}, 5 \lambda^{4}$-dipyrrolo[1,2-c:2',1'-f][1,3,2]diazaborinin10-yl)benzoate (3a)

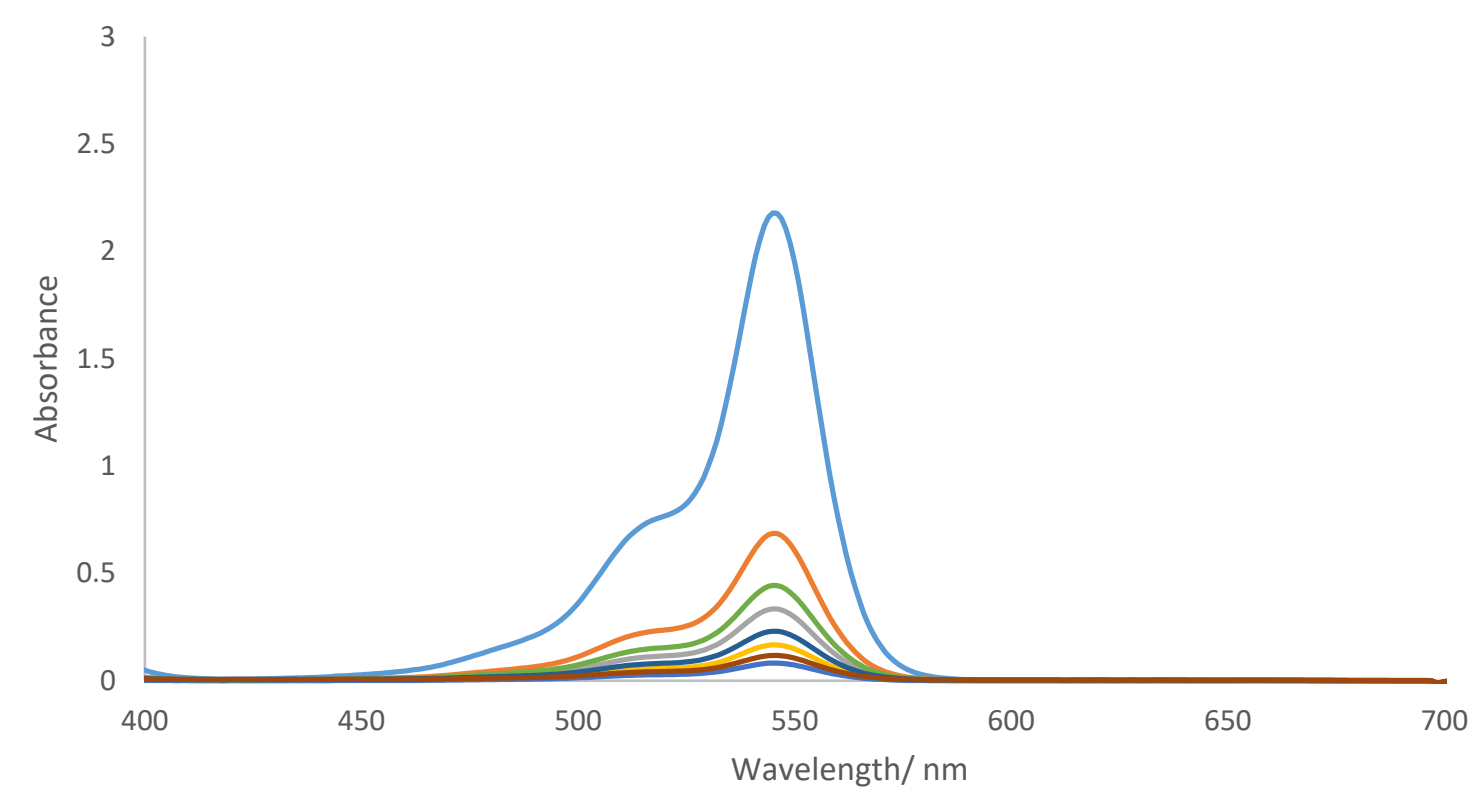

Figure S1: UV-vis spectrum of BODIPY 3a, concentrations (M): $3.46 \times 10^{-5}, 1.04 \times 10^{-5}, 6.92$ $\mathrm{x} 10^{-6}, 5.19 \times 10^{-6}, 3.46 \times 10^{-6}, 2.60 \times 10^{-6}, 1.73 \times 10^{-6}, 1.30 \times 10^{-6}$ respectively.

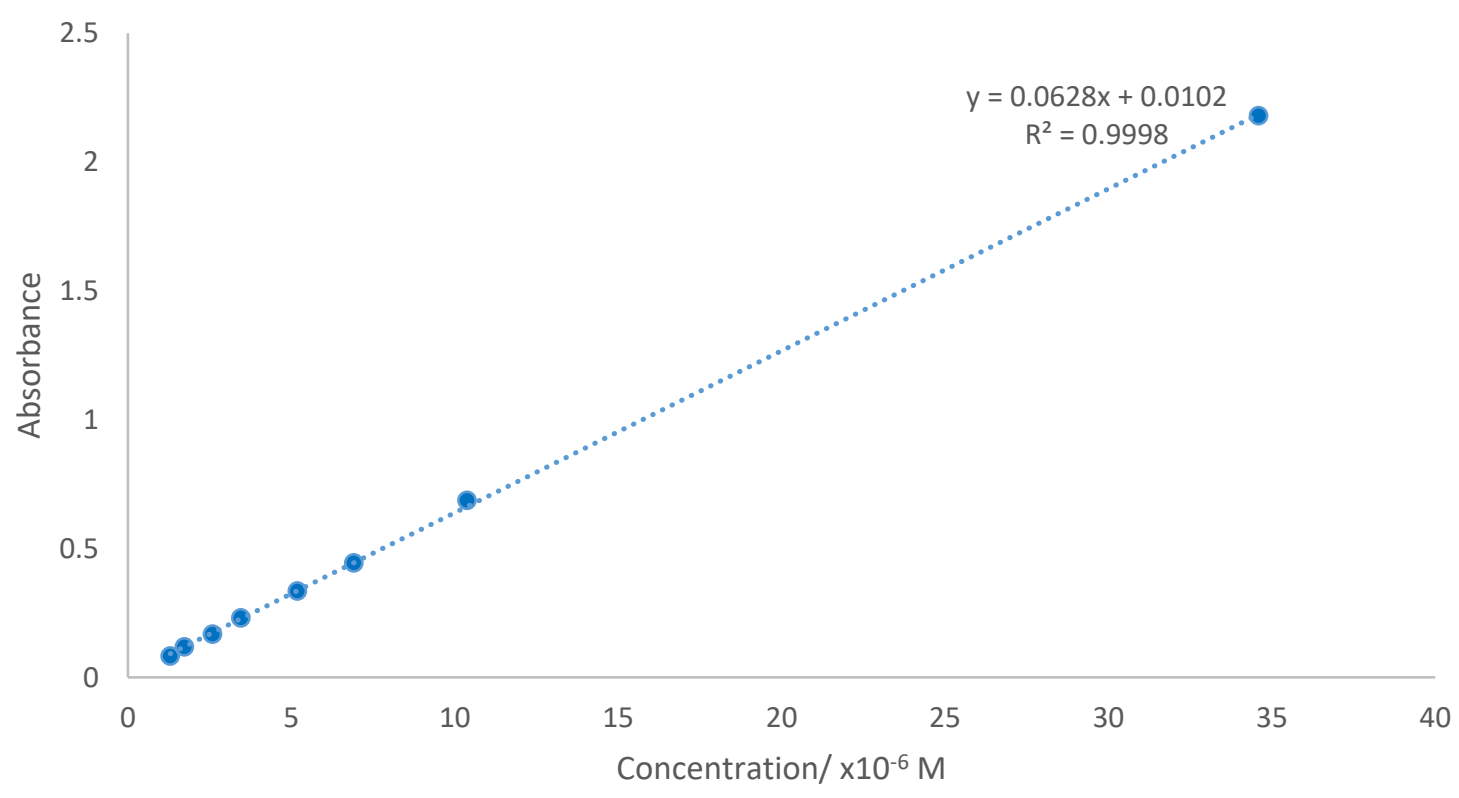

Figure S2: absorbance of BODIPY 3a at $\lambda$ max with respect to concentration, molar extinction coefficient $(\varepsilon)=63000 \mathrm{M}^{-1} \mathrm{~cm}^{-1}$. 
5,5-difluoro-3,7-diiodo-10-(3-nitrophenyl)-5H-4 $\lambda^{4}, 5 \lambda^{4}$-dipyrrolo[1,2-c:2',1'-

\section{$f][1,3,2]$ diazaborinine $(3 b)$}

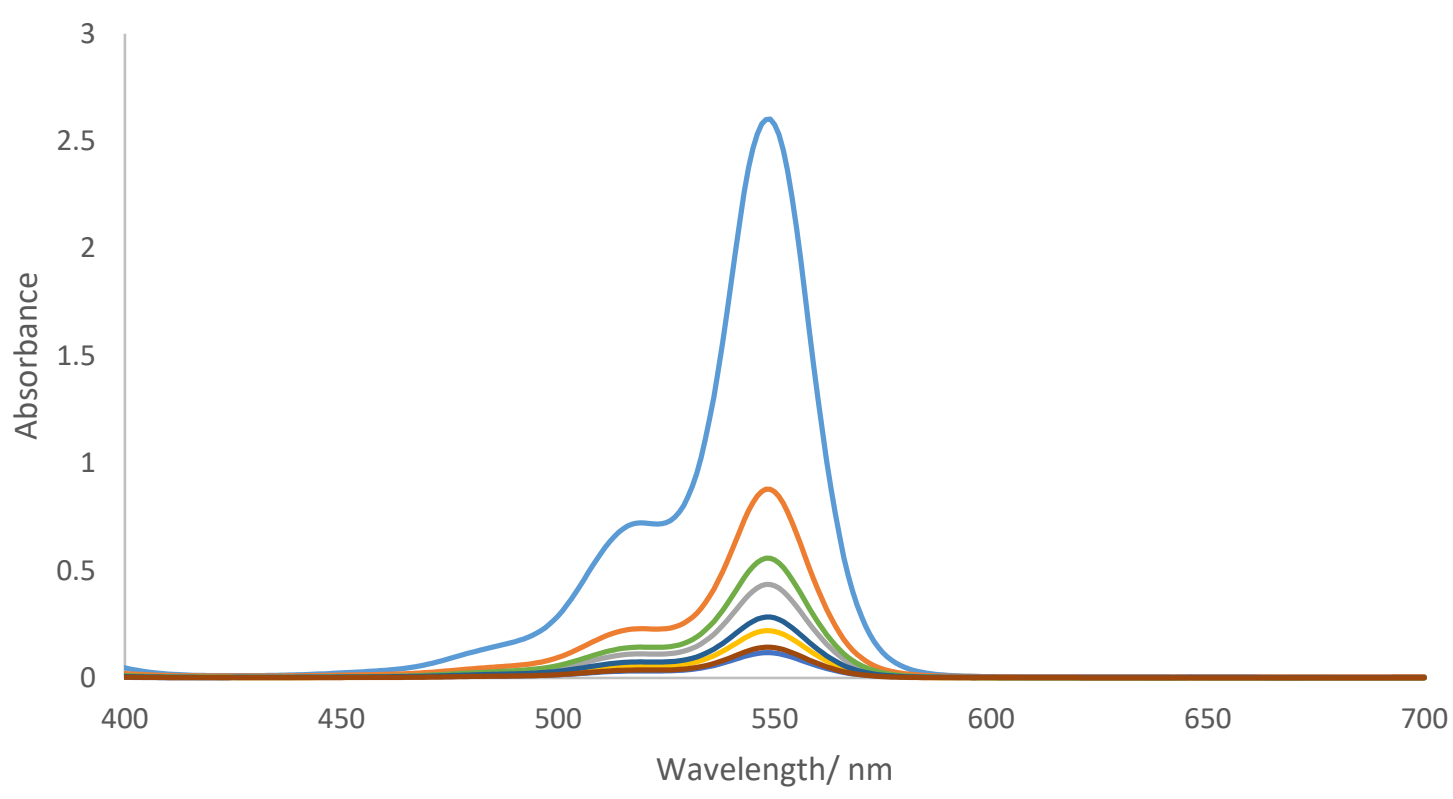

Figure S3: UV-vis spectrum of BODIPY 3b, concentrations (M): $3.54 \times 10^{-5}, 1.06 \times 10^{-5}, 7.08$ $\times 10^{-6}, 5.31 \times 10^{-6}, 3.54 \times 10^{-6}, 2.66 \times 10^{-6}, 1.77 \times 10^{-6}, 1.33 \times 10^{-6}$ respectively.

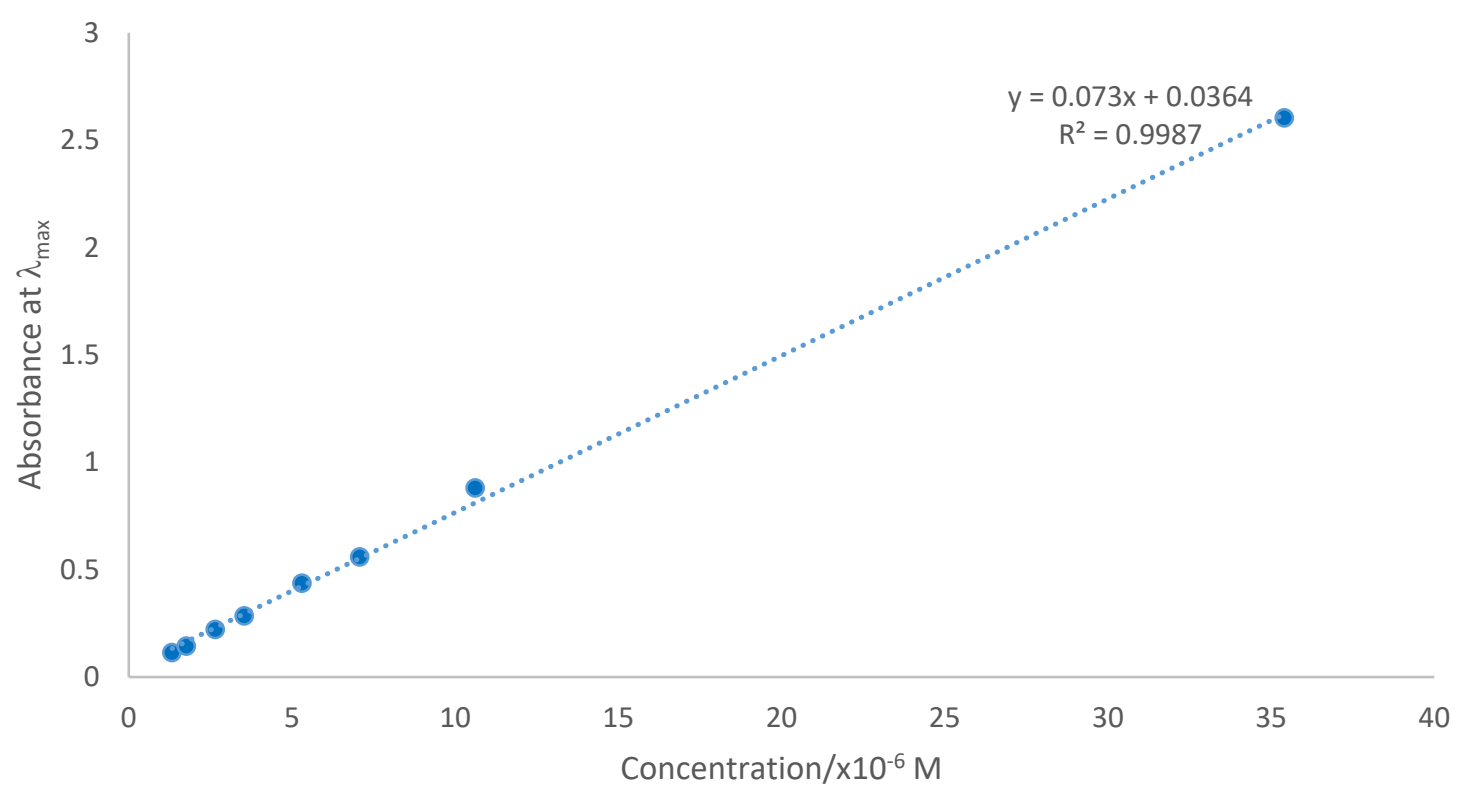

Figure S4: absorbance of BODIPY $\mathbf{3 b}$ at $\lambda$ max with respect to concentration, molar extinction coefficient $(\varepsilon)=73000 \mathrm{M}^{-1} \mathrm{~cm}^{-1}$. 
5,5-difluoro-3,7-diiodo-10-(4-methoxyphenyl)-5H-4 $\lambda^{4}, 5 \lambda^{4}$-dipyrrolo[1,2-c:2',1'-

$f][1,3,2]$ diazaborinine $(3 \mathrm{c})$

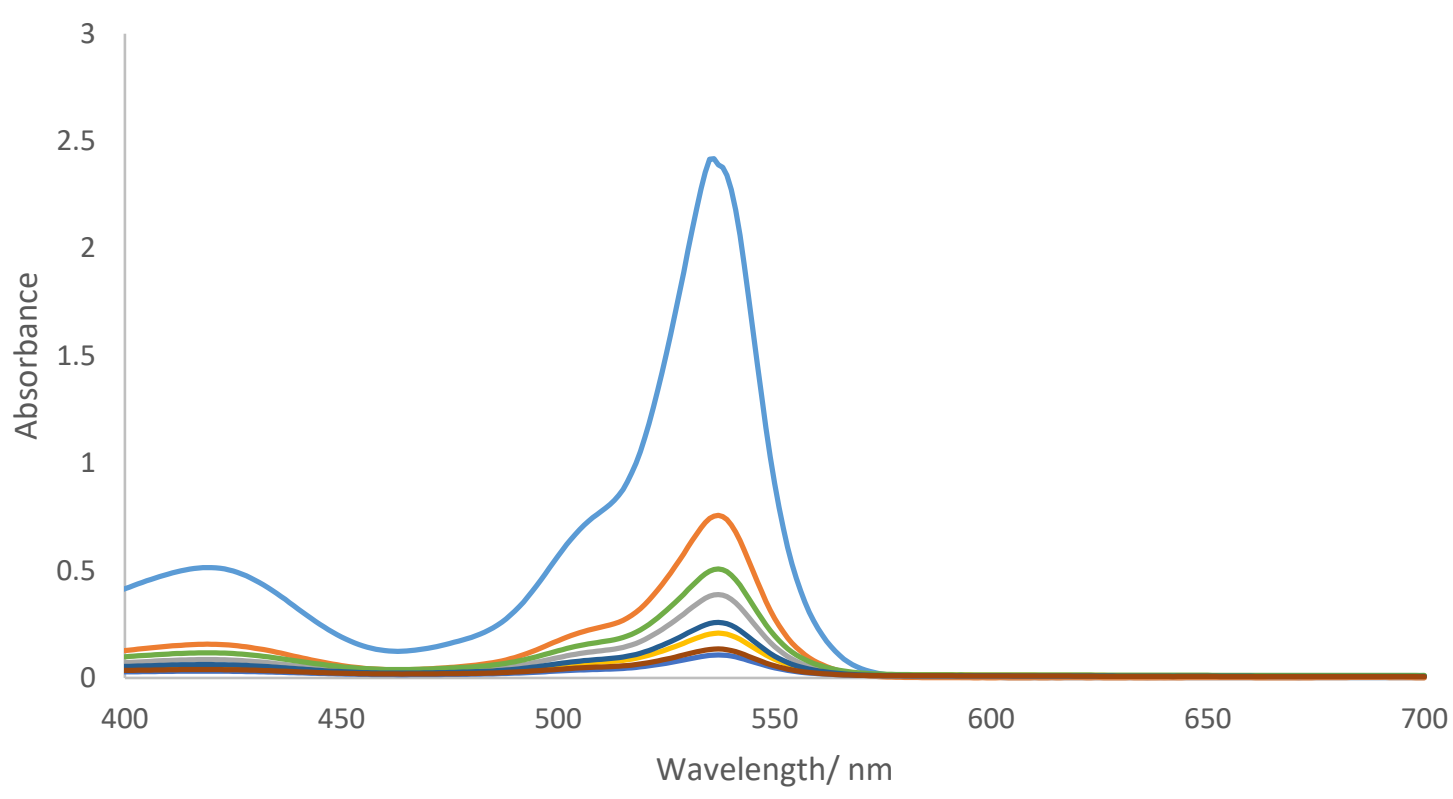

Figure S5: UV-vis spectrum of BODIPY 3c, concentrations (M): $3.64 \times 10^{-5}, 1.09 \times 10^{-5}, 7.27$ $\mathrm{x} 10^{-6}, 5.46 \times 10^{-6}, 3.64 \times 10^{-6}, 2.73 \times 10^{-6}, 1.82 \times 10^{-6}, 1.36 \times 10^{-6}$ respectively.

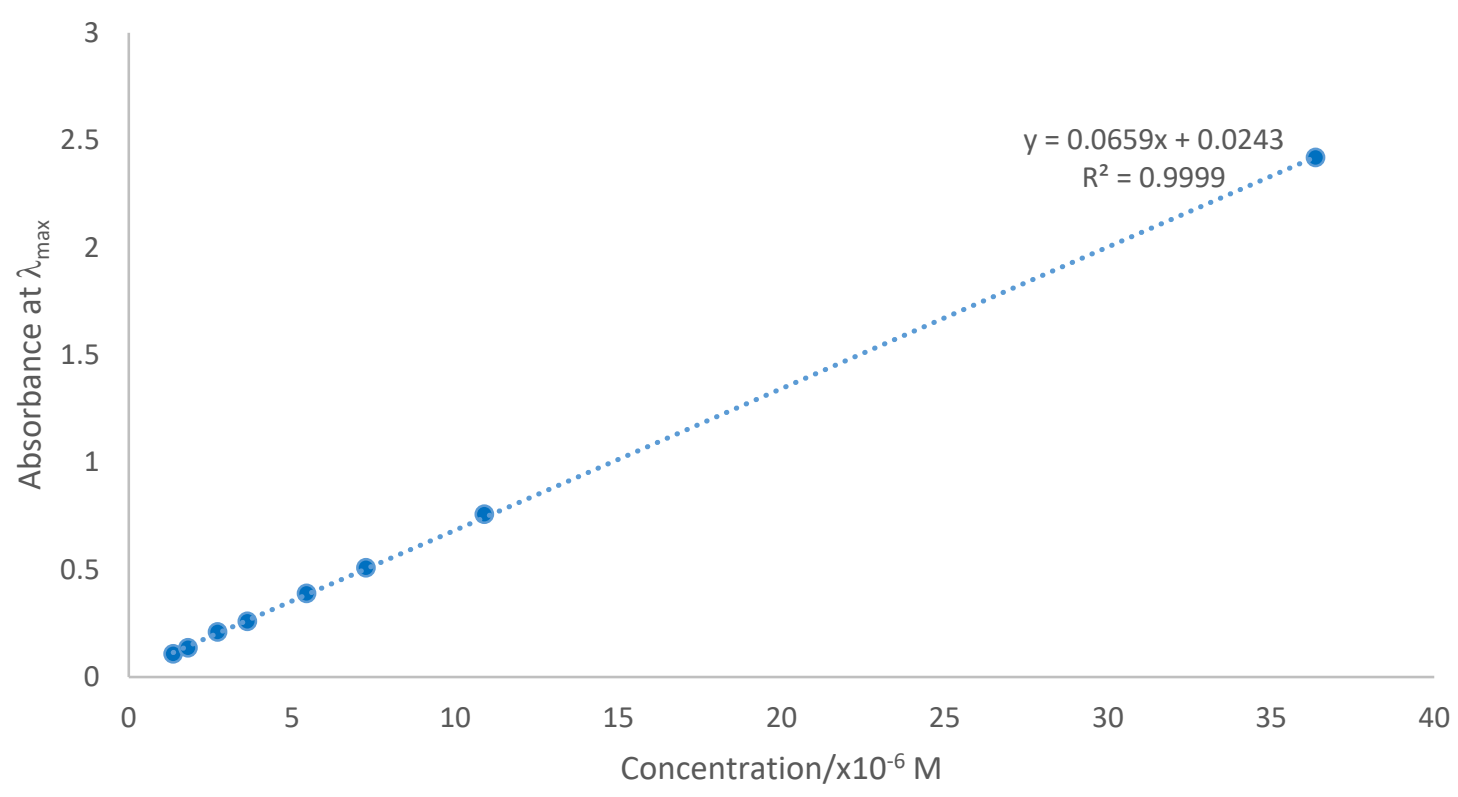

Figure S6: absorbance of BODIPY $3 \mathbf{c}$ at $\lambda \max$ with respect to concentration, molar extinction coefficient $(\varepsilon)=66000 \mathrm{M}^{-1} \mathrm{~cm}^{-1}$. 
5,5-difluoro-3,7-diiodo-10-(3-methoxyphenyl)-5H-4 $\lambda^{4}, 5 \lambda^{4}$-dipyrrolo[1,2-c:2',1'$f][1,3,2]$ diazaborinine $(3 \mathrm{~d})$

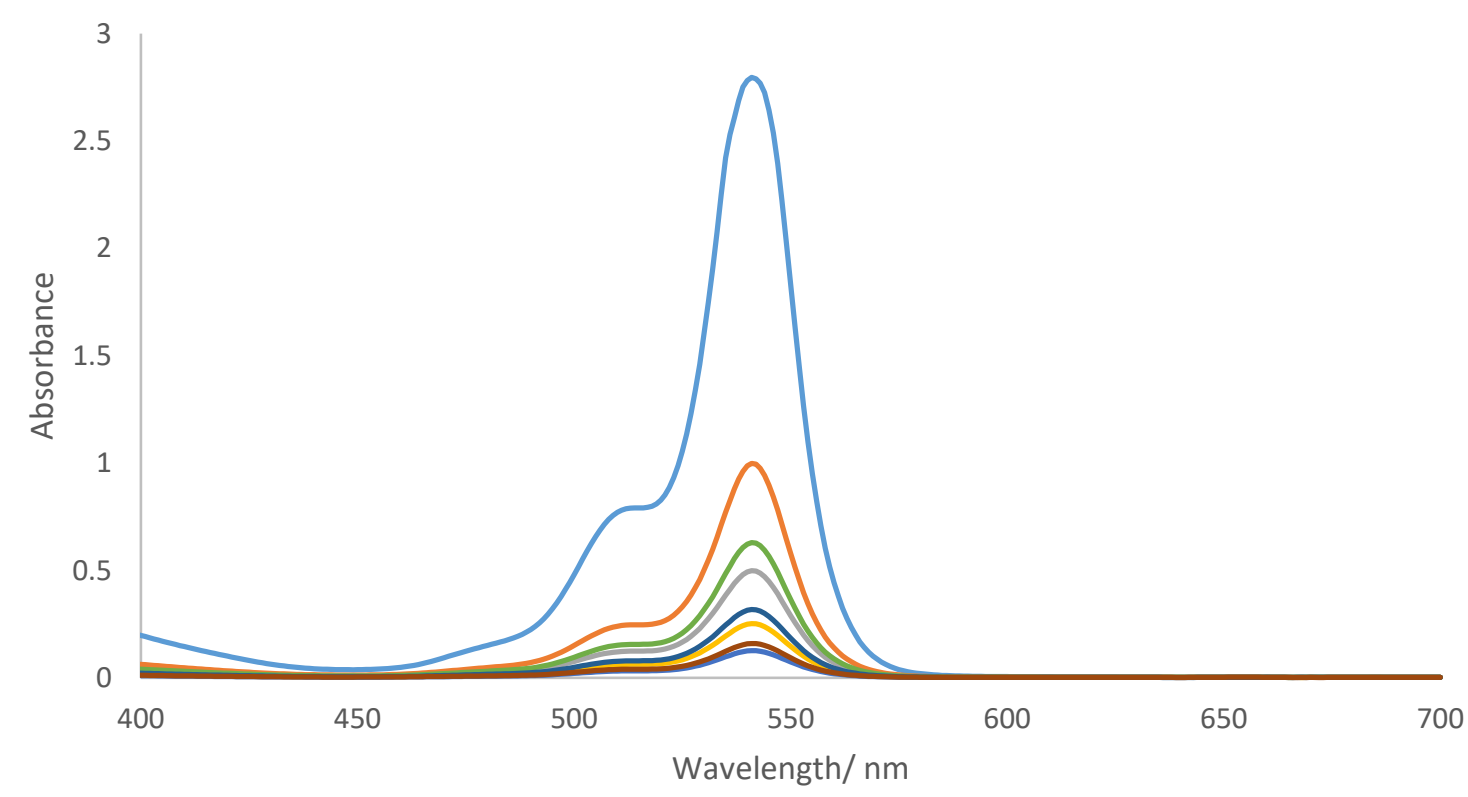

Figure S7: UV-vis spectrum of BODIPY 3d, concentrations (M): $3.64 \times 10^{-5}, 1.09 \times 10^{-5}, 7.27$ $\mathrm{x} 10^{-6}, 5.46 \times 10^{-6}, 3.64 \times 10^{-6}, 2.73 \times 10^{-6}, 1.82 \times 10^{-6}, 1.36 \times 10^{-6}$ respectively.

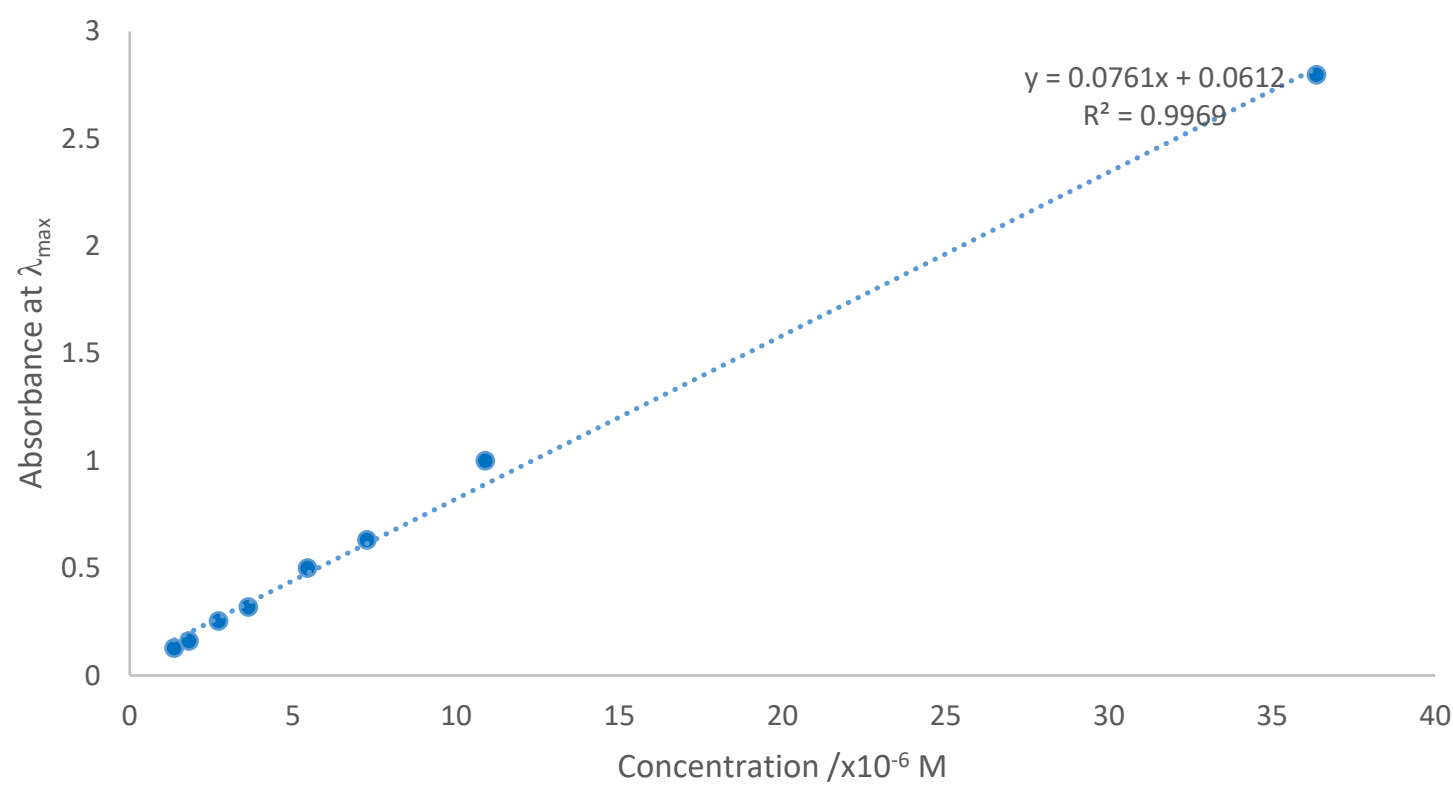

Figure S8: absorbance of BODIPY 3d at $\lambda_{\max }$ with respect to concentration, molar extinction coefficient $(\varepsilon)=76000 \mathrm{M}^{-1} \mathrm{~cm}^{-1}$. 
Methyl 4-(5,5-difluoro-3,7-diphenyl-5H-4 $\lambda^{4}, 5 \lambda^{4}$-dipyrrolo[1,2-c:2',1'-

$f][1,3,2]$ diazaborinin-10-yl)benzoate (4)

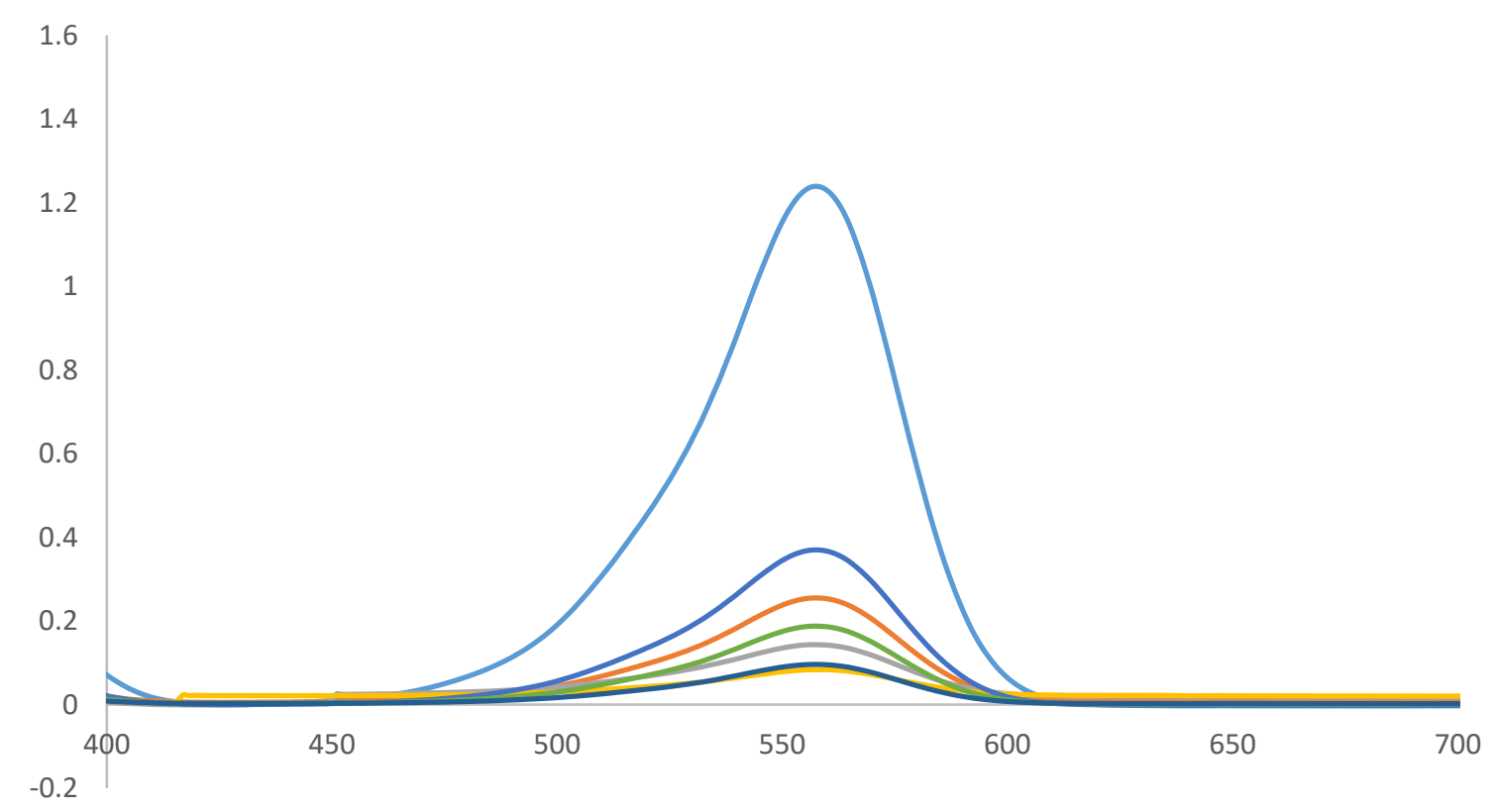

Figure S9: UV-vis spectrum of BODIPY 4, concentrations (M): $2.10 \times 10^{-5}, 6.30 \times 10^{-6}, 4.20$ $\mathrm{x} 10^{-6}, 3.15 \times 10^{-6}, 2.10 \times 10^{-6}, 1.58 \times 10^{-6}, 1.05 \times 10^{-6}$ respectively.

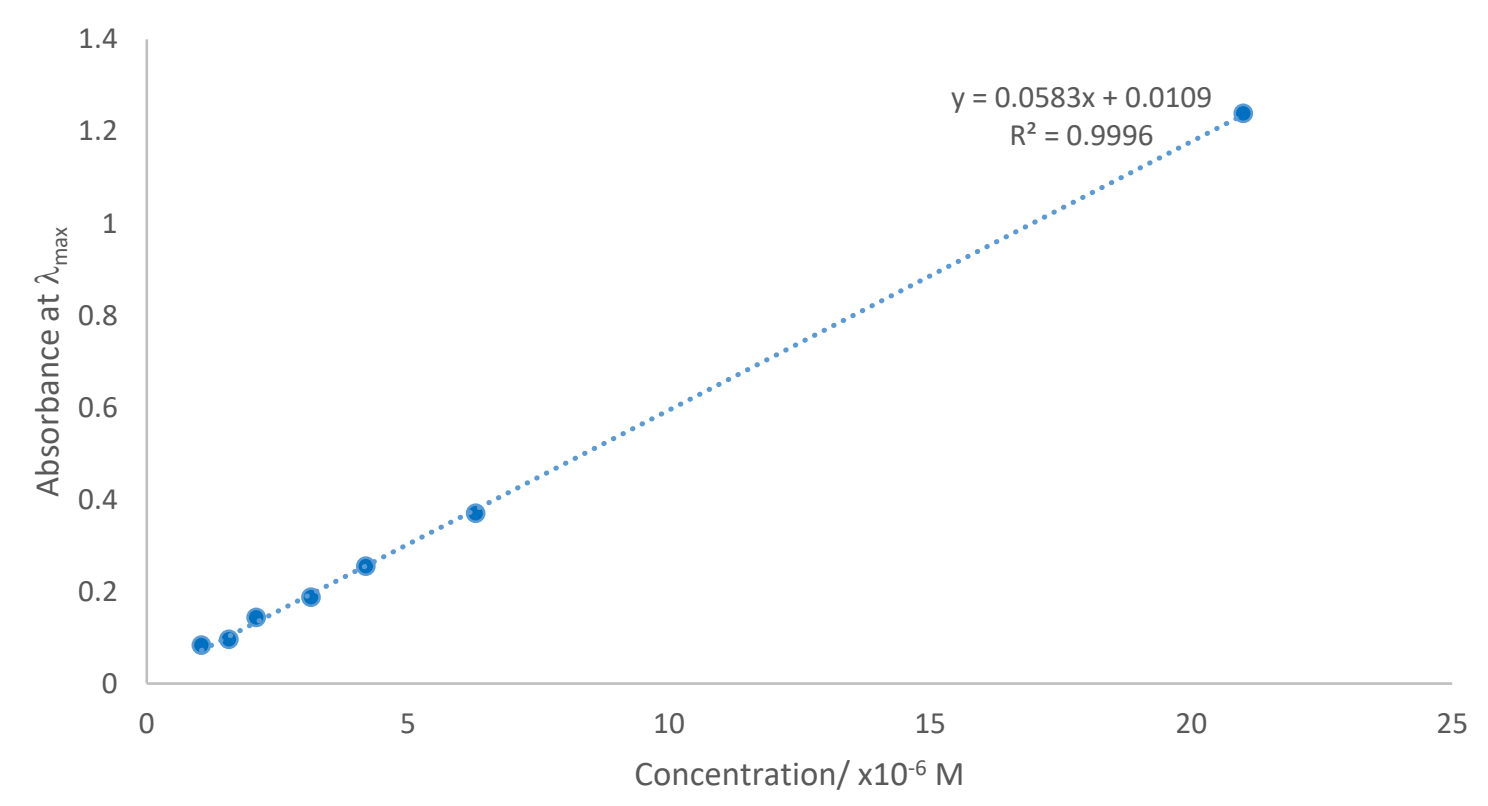

Figure S10: absorbance of BODIPY 4 at $\lambda$ max with respect to concentration, molar extinction coefficient $(\varepsilon)=58000 \mathrm{M}^{-1} \mathrm{~cm}^{-1}$. 


\section{$f][1,3,2]$ diazaborinin-10-yl)benzoate (5)}

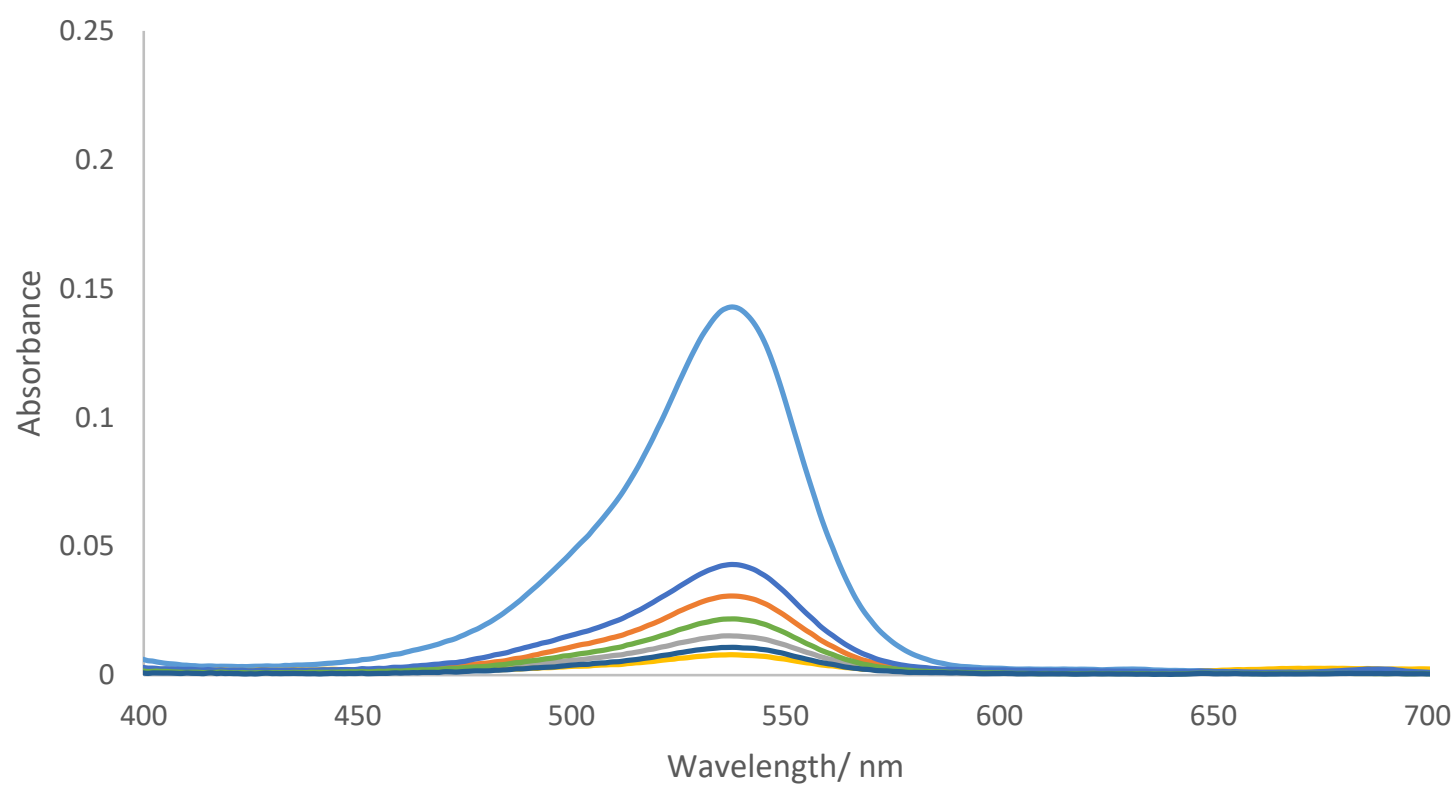

Figure S11: UV-vis spectrum of BODIPY 5, concentrations (M): $2.07 \times 10^{-6}, 6.21 \times 10^{-7}, 4.16$ $\mathrm{x} 10^{-7}, 3.11 \times 10^{-7}, 2.08 \times 10^{-7}, 1.55 \times 10^{-7}, 1.04 \times 10^{-7}$ respectively.

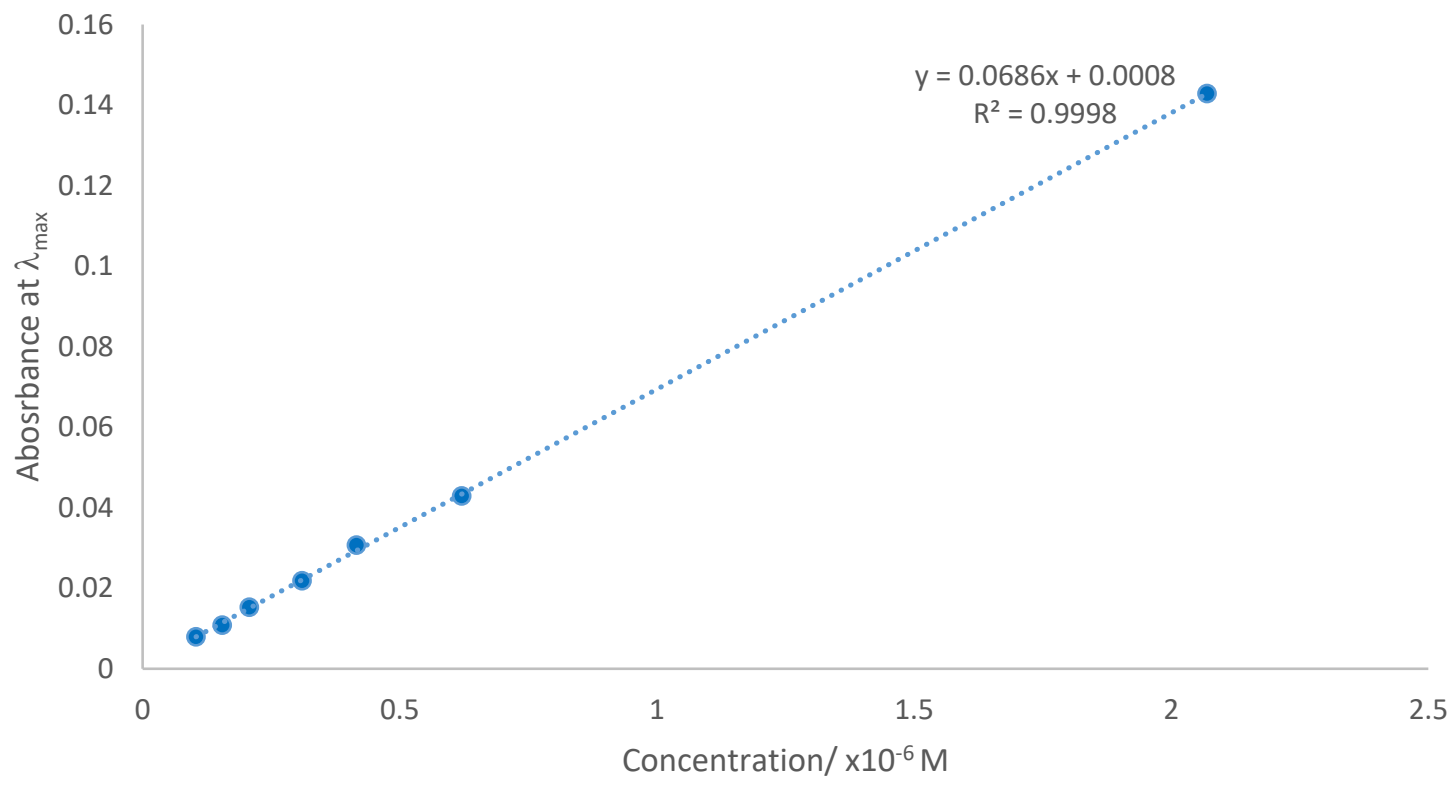

Figure S12: absorbance of BODIPY 5 at $\lambda_{\max }$ with respect to concentration, molar extinction coefficient $(\varepsilon)=69000 \mathrm{M}^{-1} \mathrm{~cm}^{-1}$. 
Methyl 4-(5,5-difluoro-3,7-di((E)-styryl)-5H-4 $\lambda^{4}, 5 \lambda^{4}$-dipyrrolo[1,2-c:2',1'-

$f][1,3,2]$ diazaborinin-10-yl)benzoate (6)

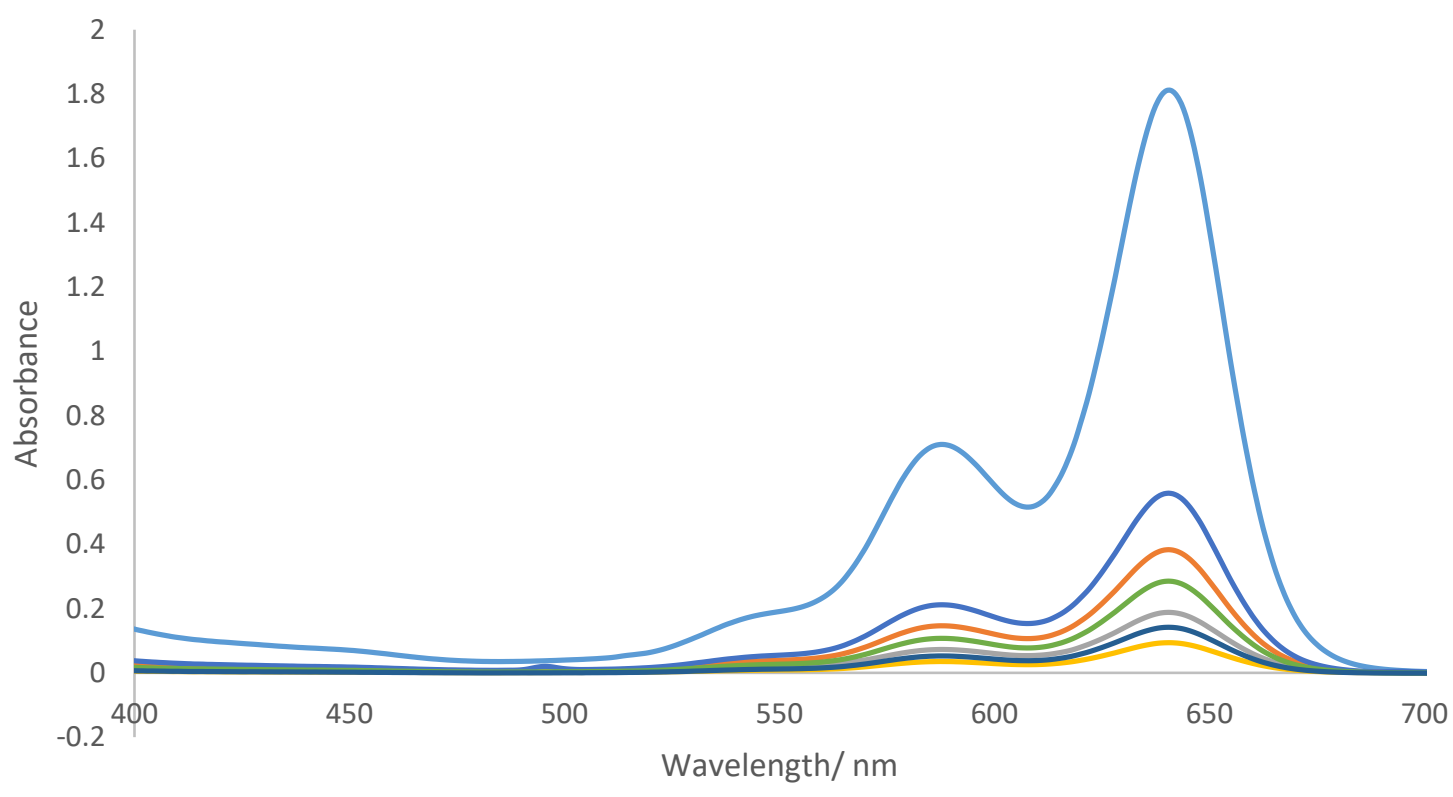

Figure S13: UV-vis spectrum of BODIPY 6, concentrations (M): $1.89 \times 10^{-5}, 5.66 \times 10^{-6}, 3.77$ $\times 10^{-6}, 2.83 \times 10^{-6}, 1.89 \times 10^{-6}, 1.41 \times 10^{-6}, 9.43 \times 10^{-7}$ respectively.

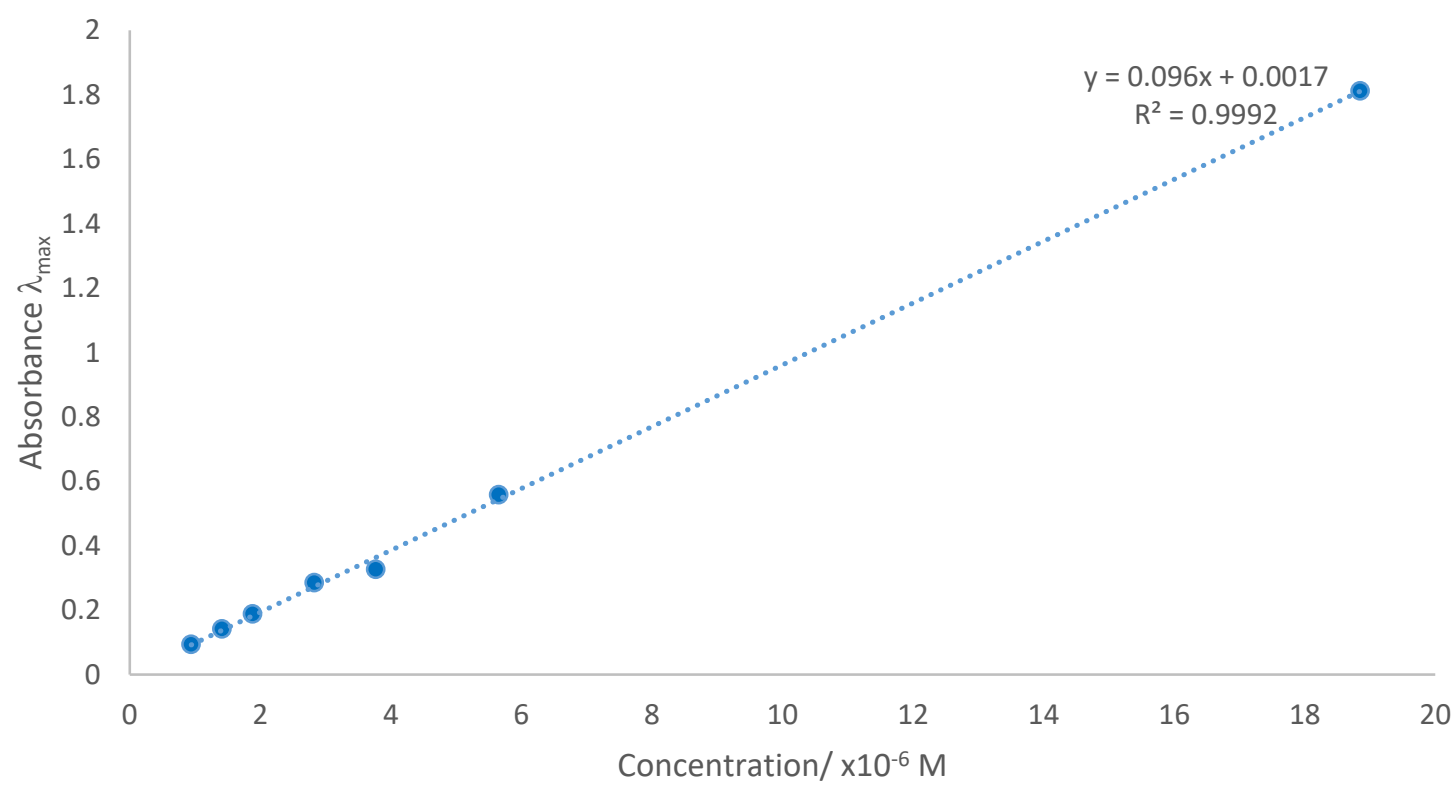

Figure S14: absorbance of BODIPY 6 at $\lambda_{\max }$ with respect to concentration, molar extinction coefficient $(\varepsilon)=96000 \mathrm{M}^{-1} \mathrm{~cm}^{-1}$. 


\section{$f][1,3,2]$ diazaborinin-10-yl)benzoate (7)}

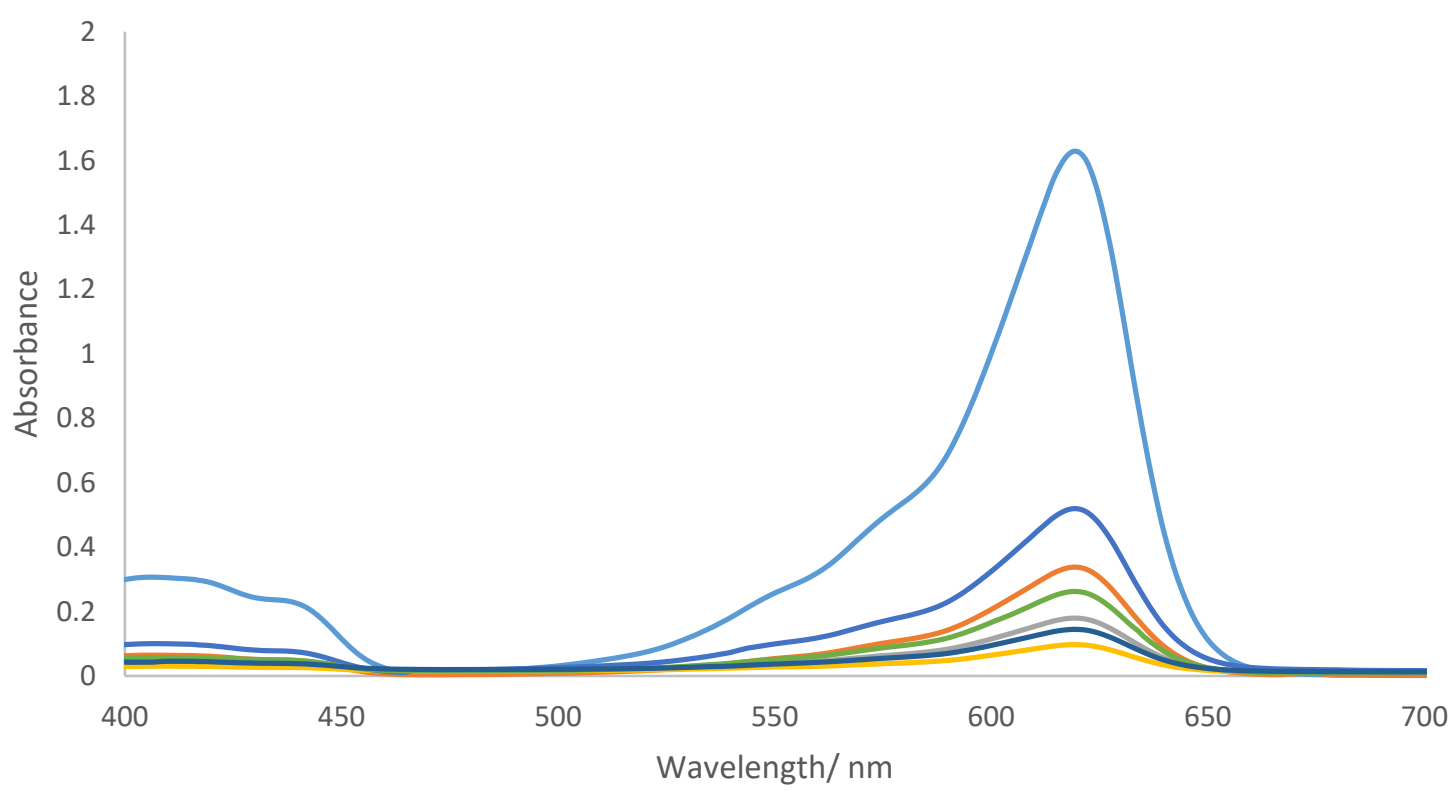

Figure S15: UV-vis spectrum of BODIPY 7, concentrations (M): $1.90 \times 10^{-5}, 5.70 \times 10^{-6}, 3.80$ $\mathrm{x} 10^{-6}, 2.85 \times 10^{-6}, 1.90 \times 10^{-6}, 1.43 \times 10^{-6}, 9.50 \times 10^{-7}$ respectively.

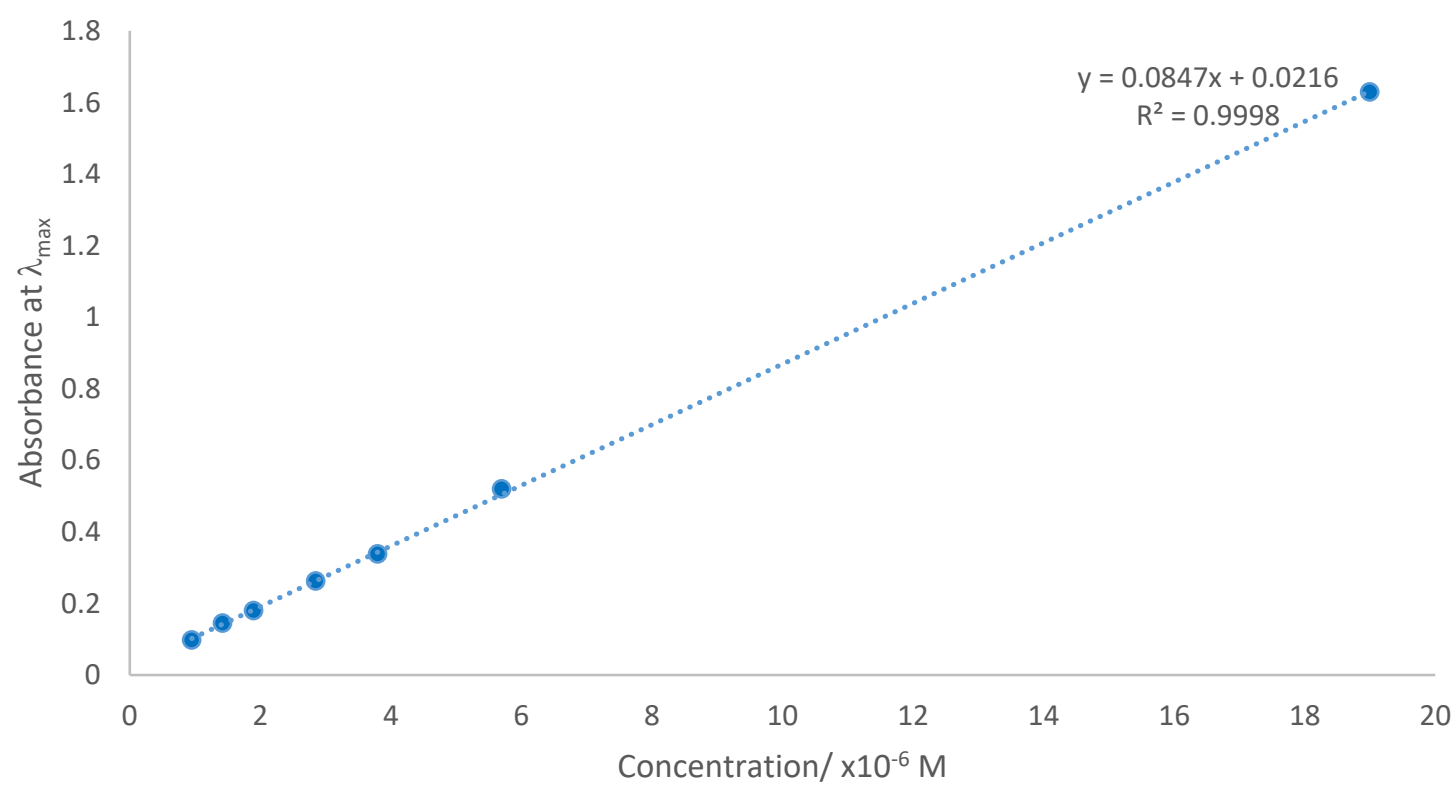

Figure S16: absorbance of BODIPY 7 at $\lambda$ max with respect to concentration, molar extinction coefficient $(\varepsilon)=85000 \mathrm{M}^{-1} \mathrm{~cm}^{-1}$. 
Methyl 4-(3-chloro-5,5-difluoro-7-(phenylethynyl)-5H-4 $\lambda^{4}, 5 \lambda^{4}$-dipyrrolo[1,2-c:2',1'$f][1,3,2]$ diazaborinin-10-yl)benzoate (8)

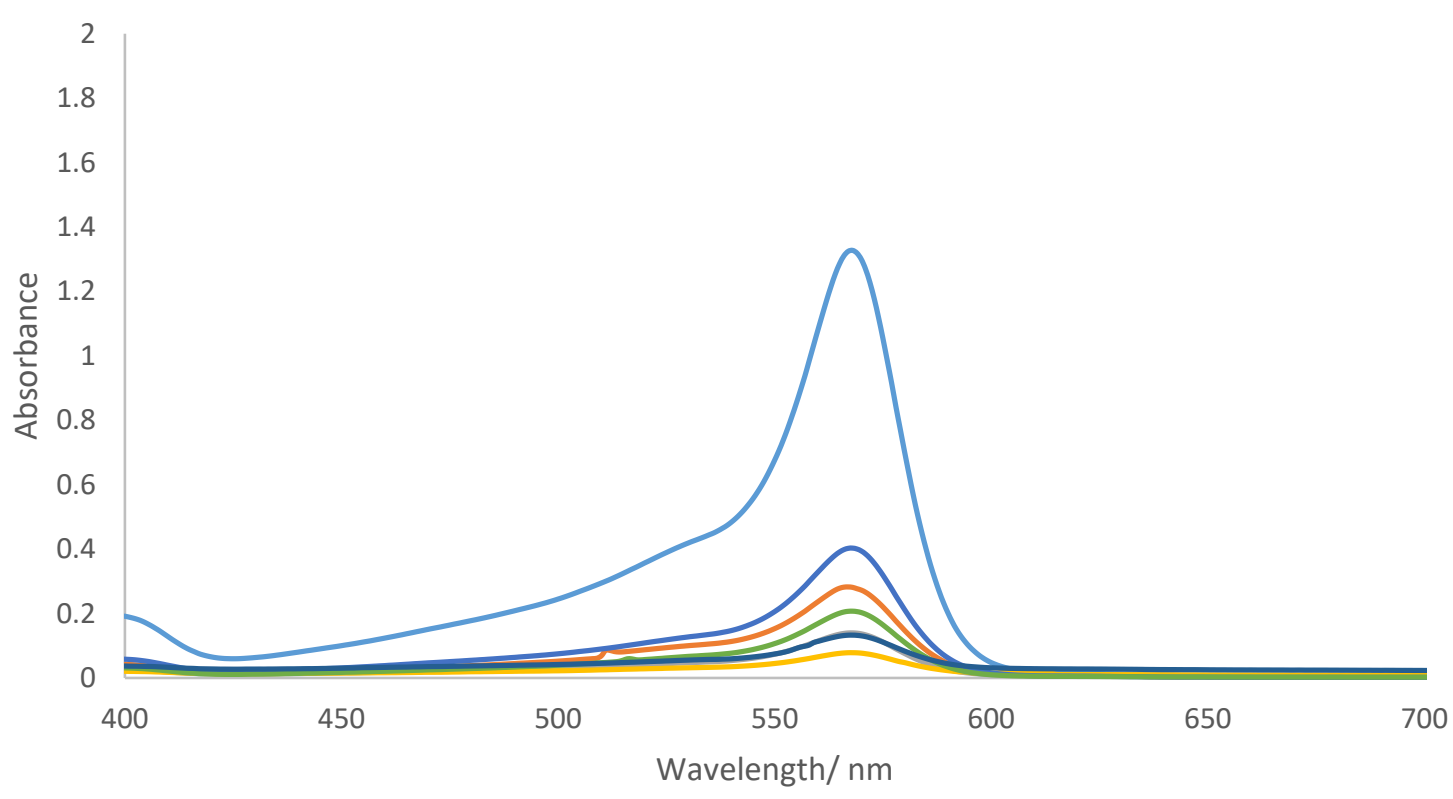

Figure S17: UV-vis spectrum of BODIPY 8, concentrations (M): $2.17 \times 10^{-5}, 6.51 \times 10^{-6}, 4.34$ $\mathrm{x} 10^{-6}, 3.26 \times 10^{-6}, 2.17 \times 10^{-6}, 1.62 \times 10^{-6}, 1.09 \times 10^{-6}$ respectively.

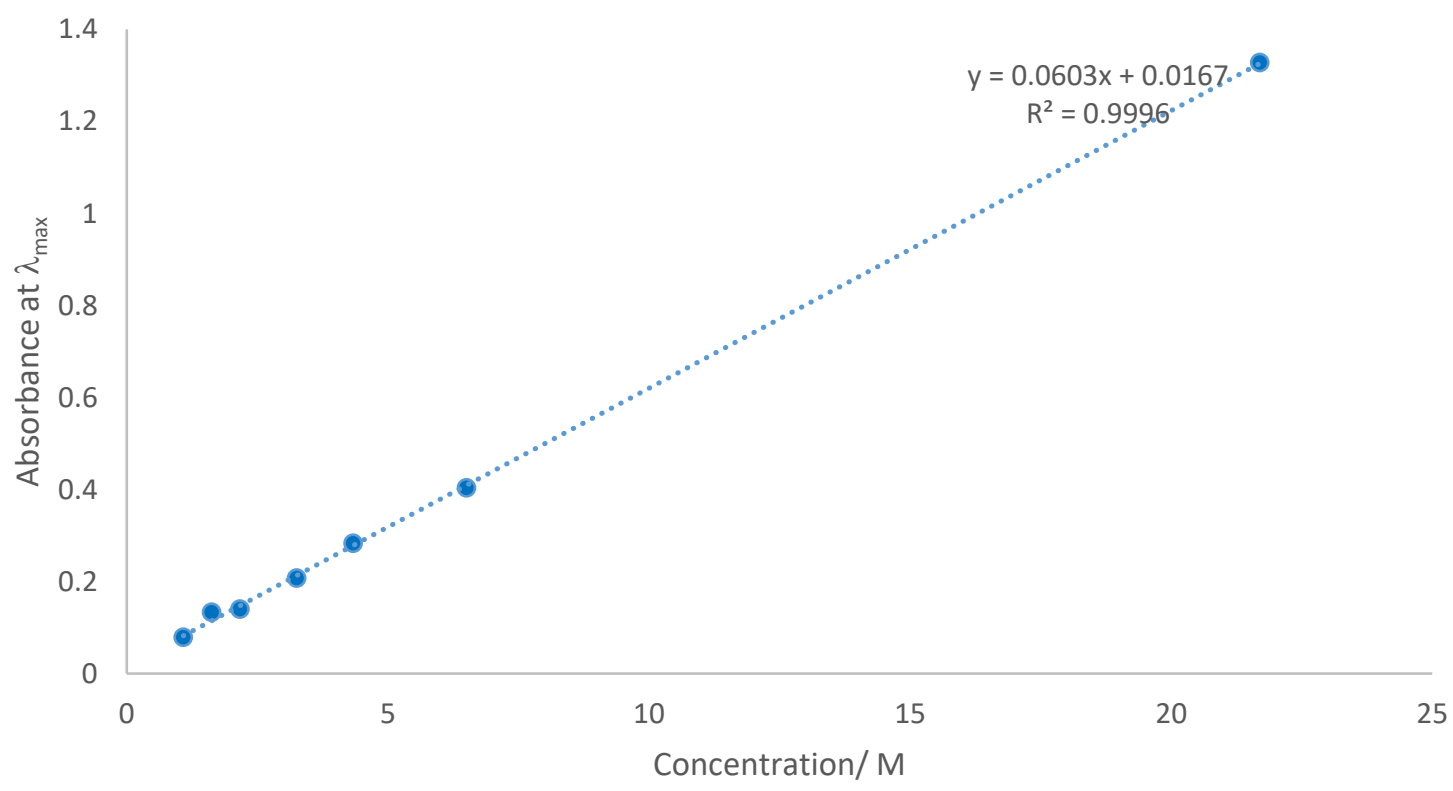

Figure S18: absorbance of BODIPY 8 at $\lambda_{\max }$ with respect to concentration, molar extinction coefficient $(\varepsilon)=60000 \mathrm{M}^{-1} \mathrm{~cm}^{-1}$. 


\section{$f][1,3,2]$ diazaborinin-10-yl)benzoate (9)}

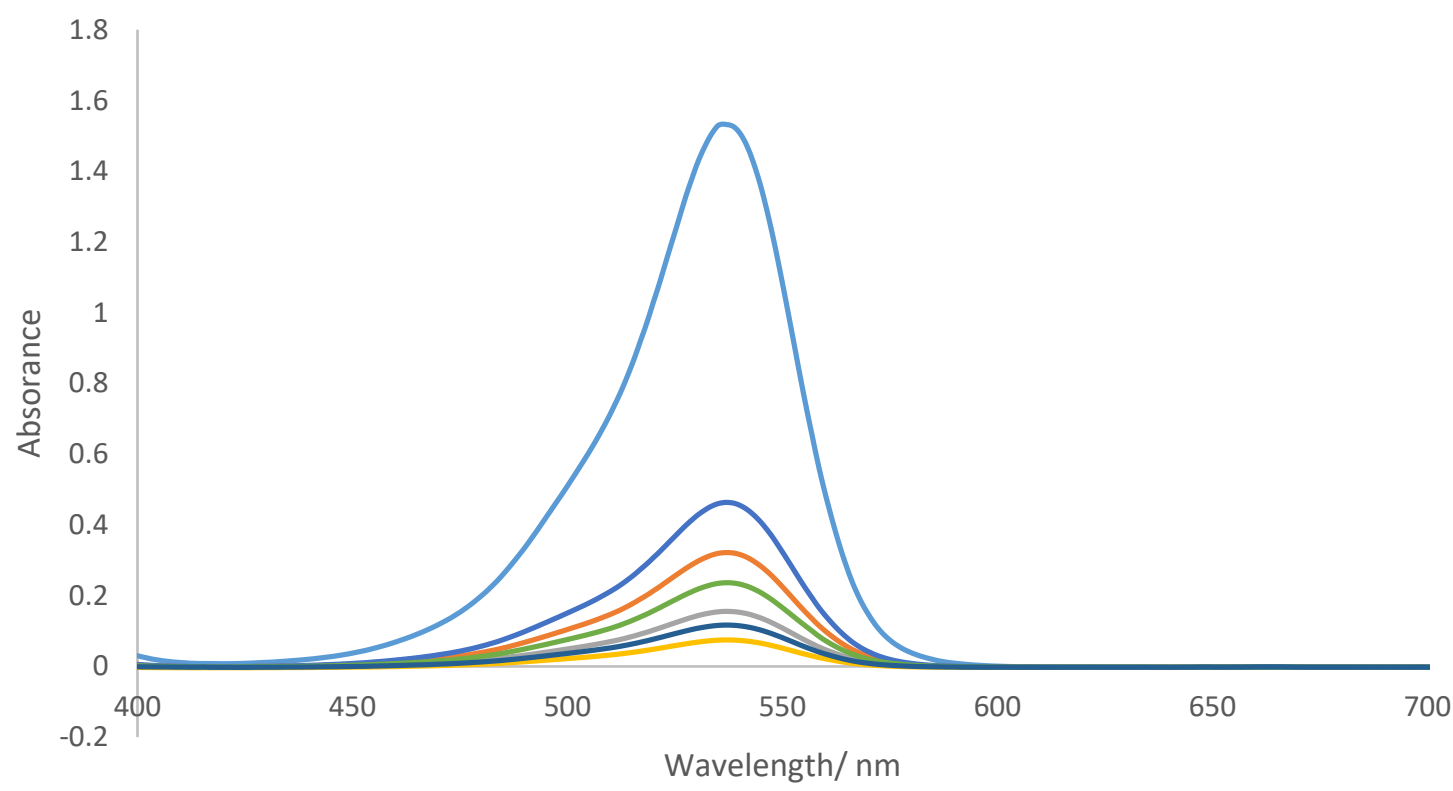

Figure S19: UV-vis spectrum of BODIPY 9, concentrations (M): $2.29 \times 10^{-5}, 6.87 \times 10^{-6}, 4.58$ $\mathrm{x} 10^{-6}, 3.44 \times 10^{-6}, 2.29 \times 10^{-6}, 1.72 \times 10^{-6}, 1.15 \times 10^{-6}$ respectively.

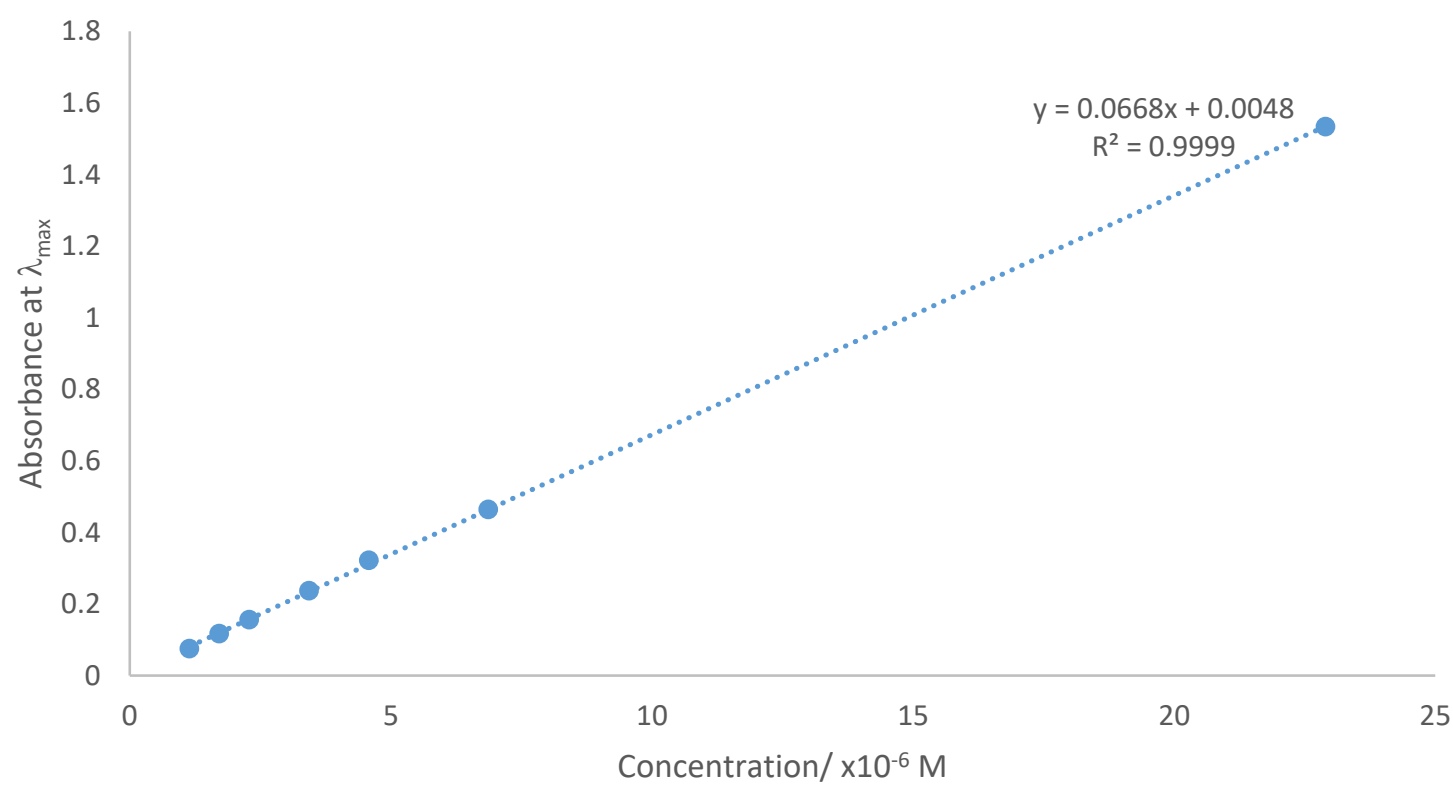

Figure S20: absorbance of BODIPY 9 at $\lambda_{\max }$ with respect to concentration, molar extinction coefficient $(\varepsilon)=67000 \mathrm{M}^{-1} \mathrm{~cm}^{-1}$. 


\section{Normalised UV/Vis absorption and emission spectra}

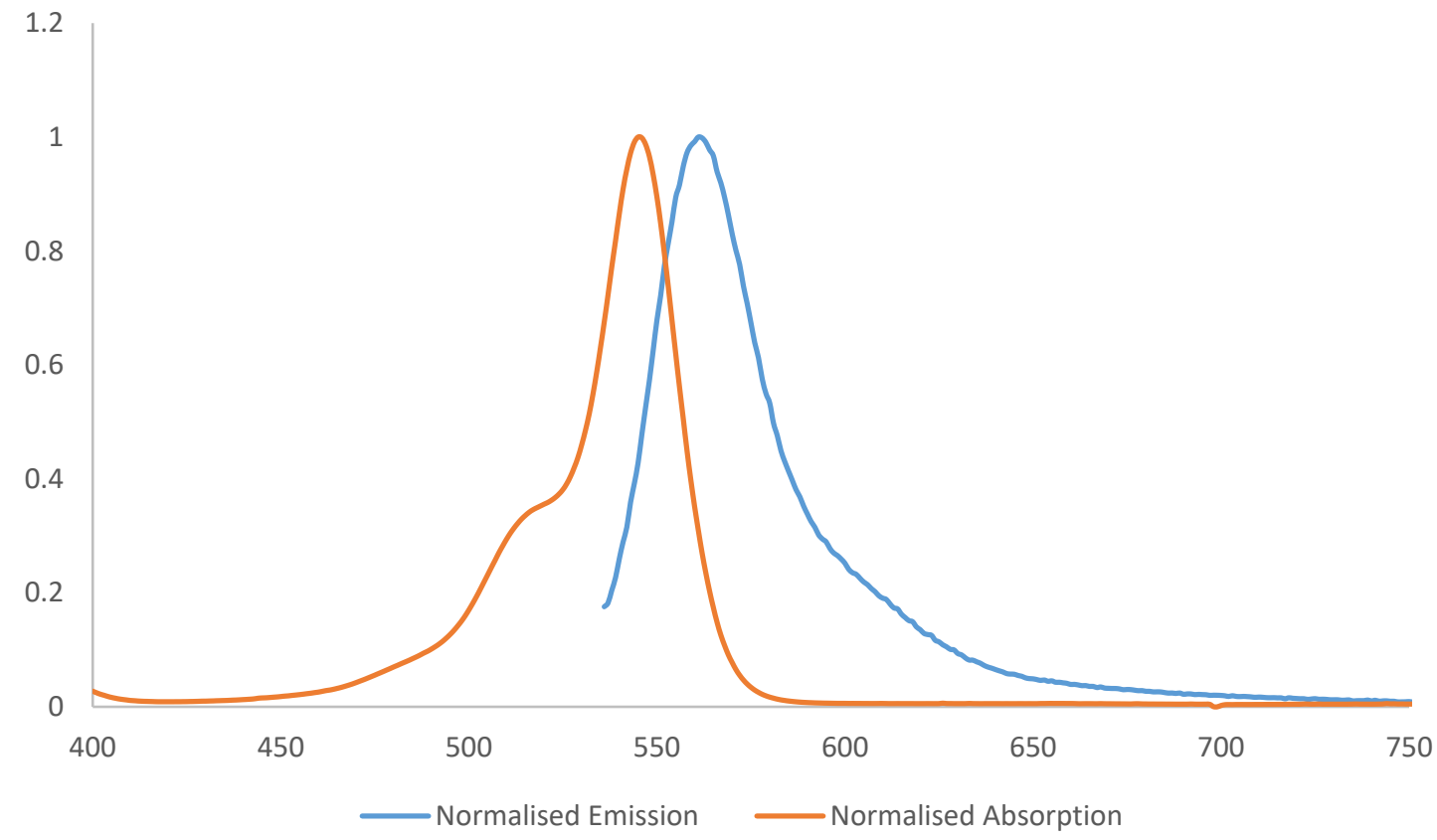

Figure S21: Normalised absorption and emission spectra of BODIPY 3a (DCM).

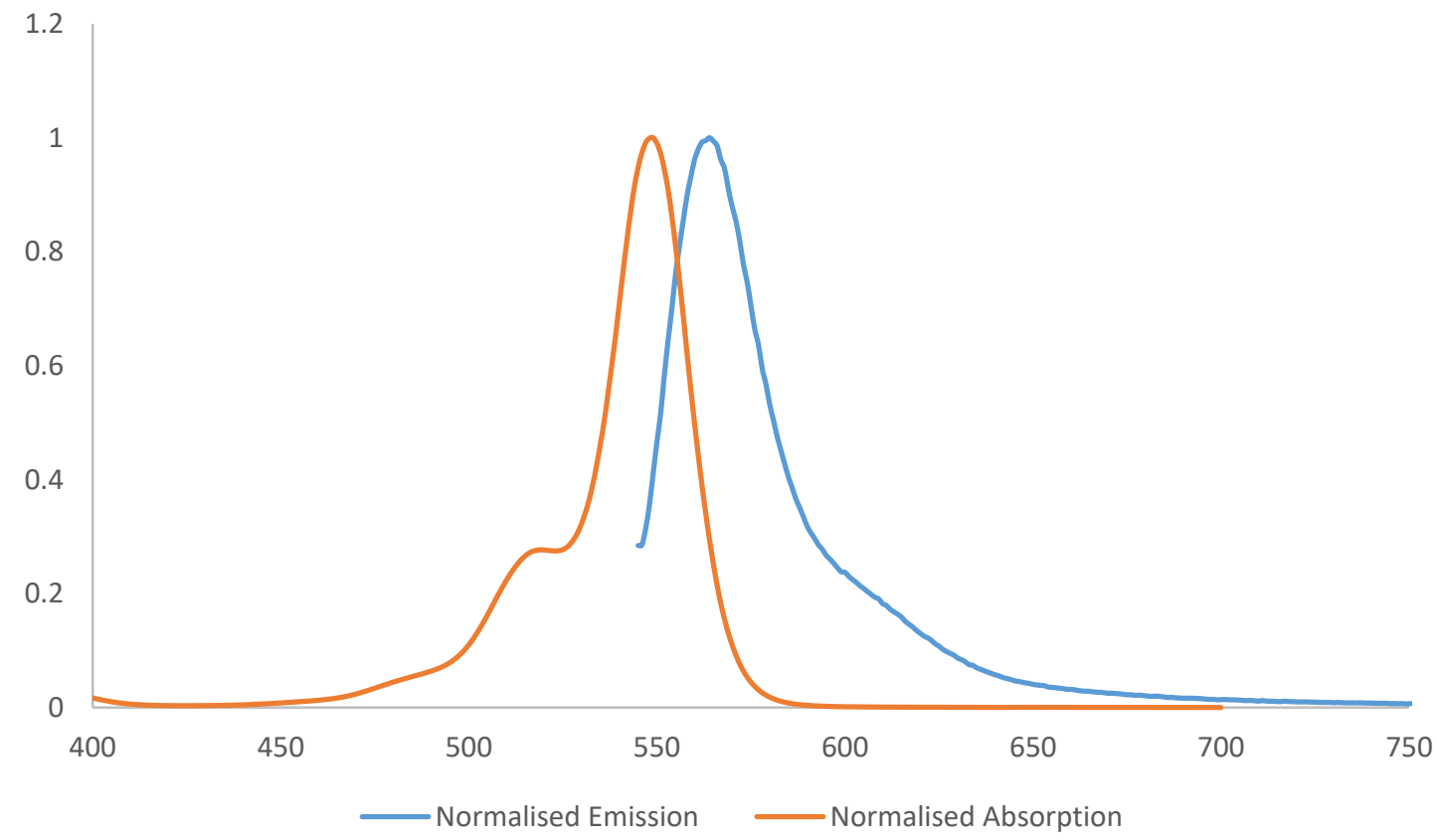

Figure S22: Normalised absorption and emission spectra of BODIPY 3b (DCM). 


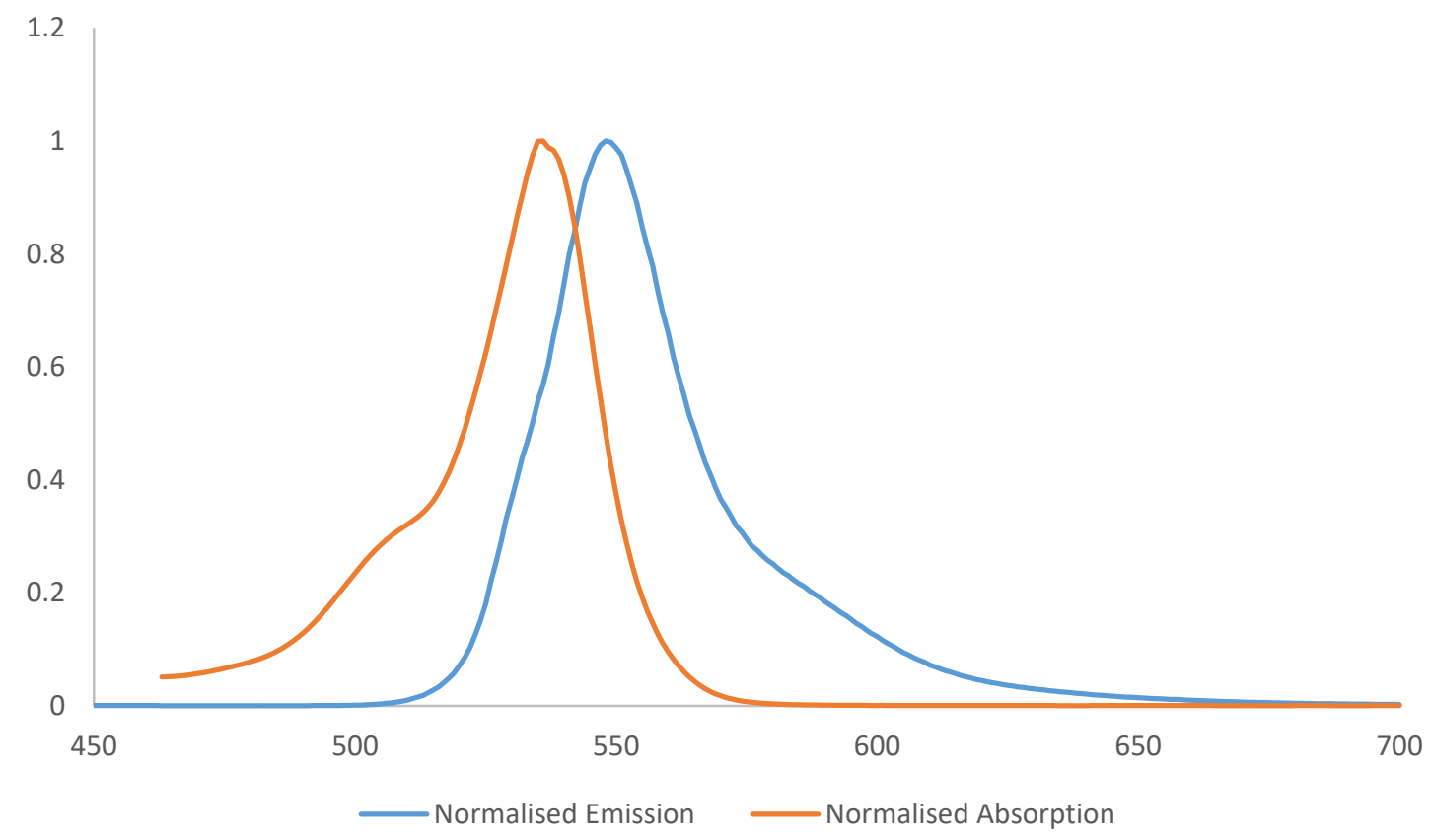

Figure S23: Normalised absorption and emission spectra of BODIPY 3c (DCM).

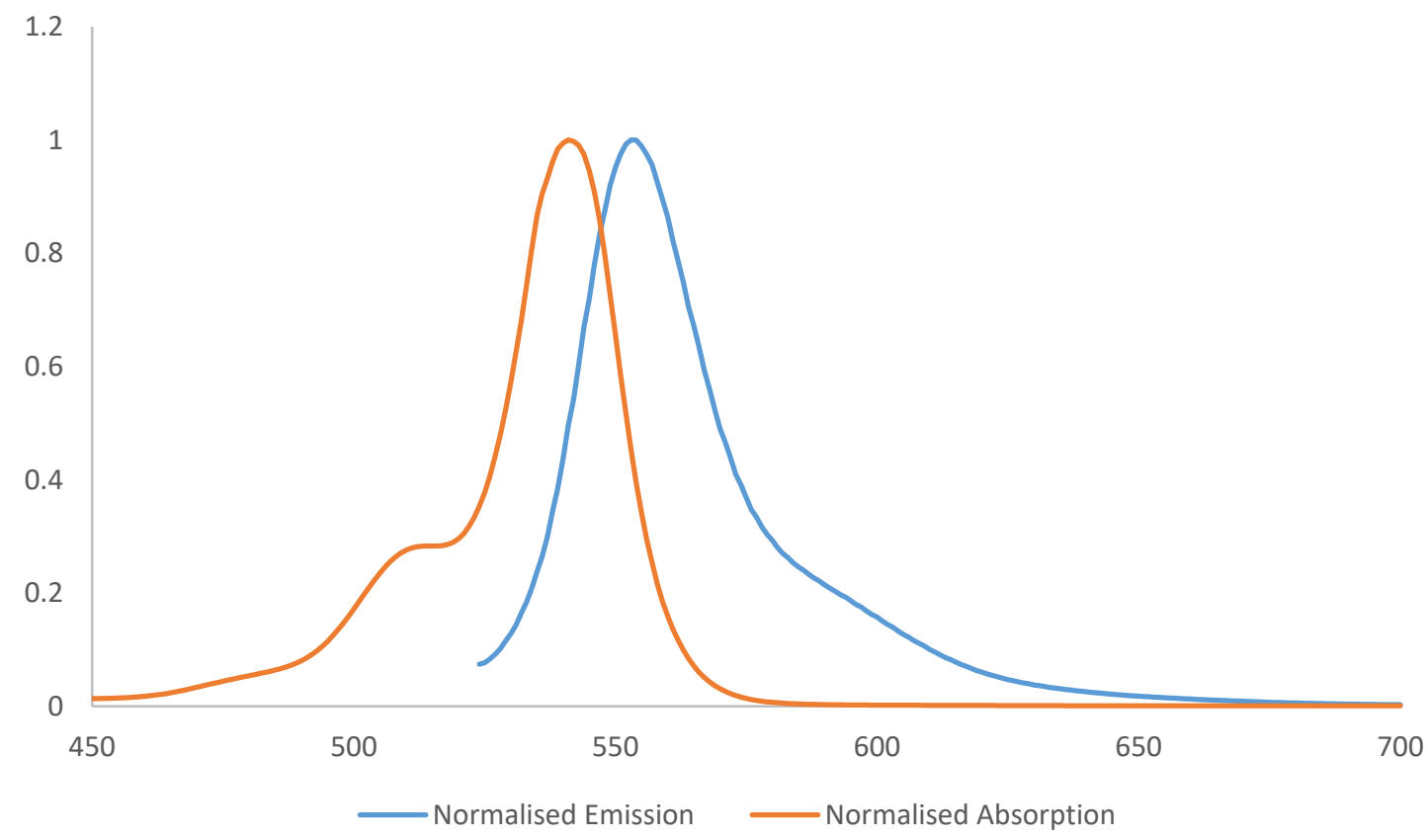

Figure S24: Normalised absorption and emission spectra of BODIPY 3d (DCM). 


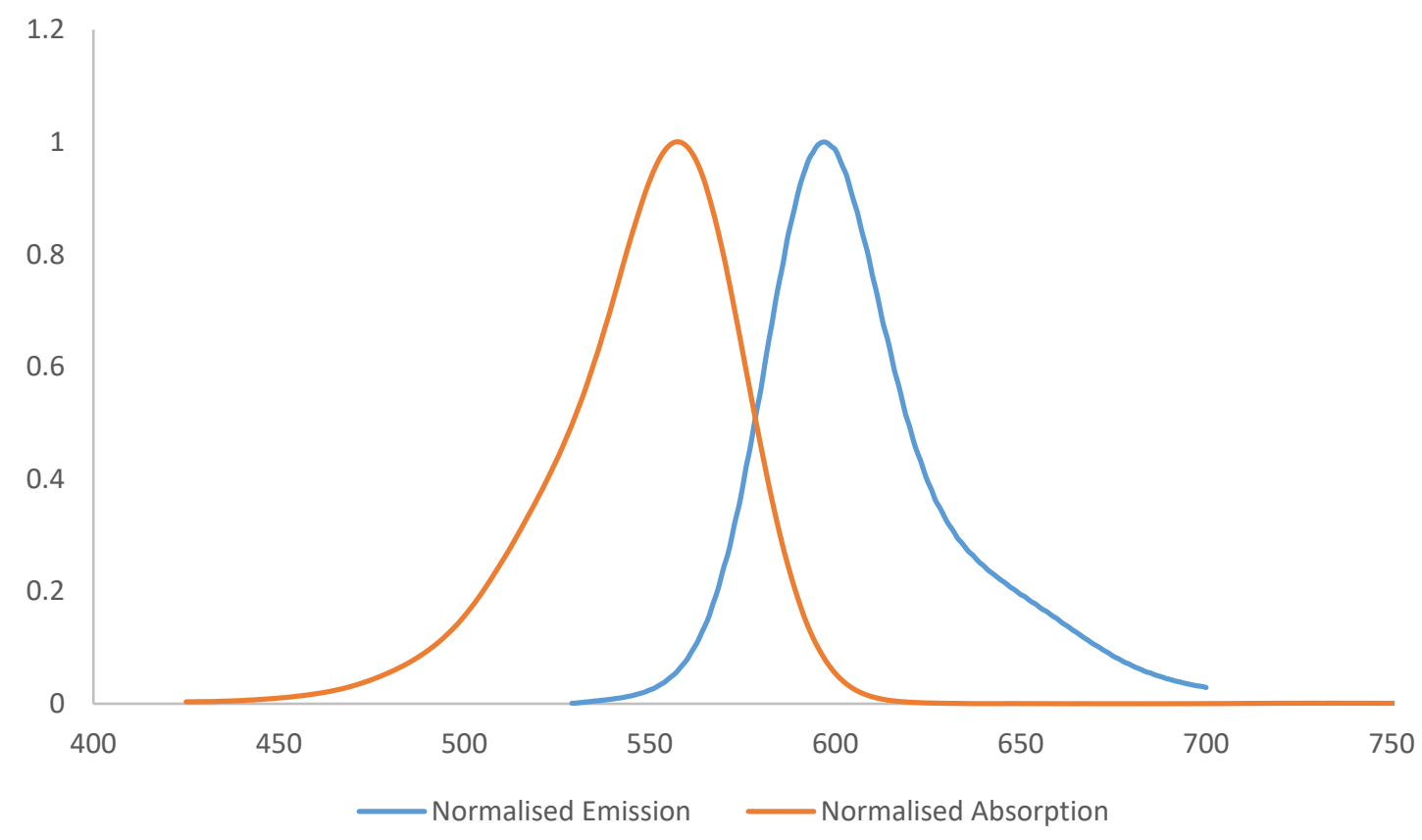

Figure S25: Normalised absorption and emission spectra of BODIPY 4 (DCM).

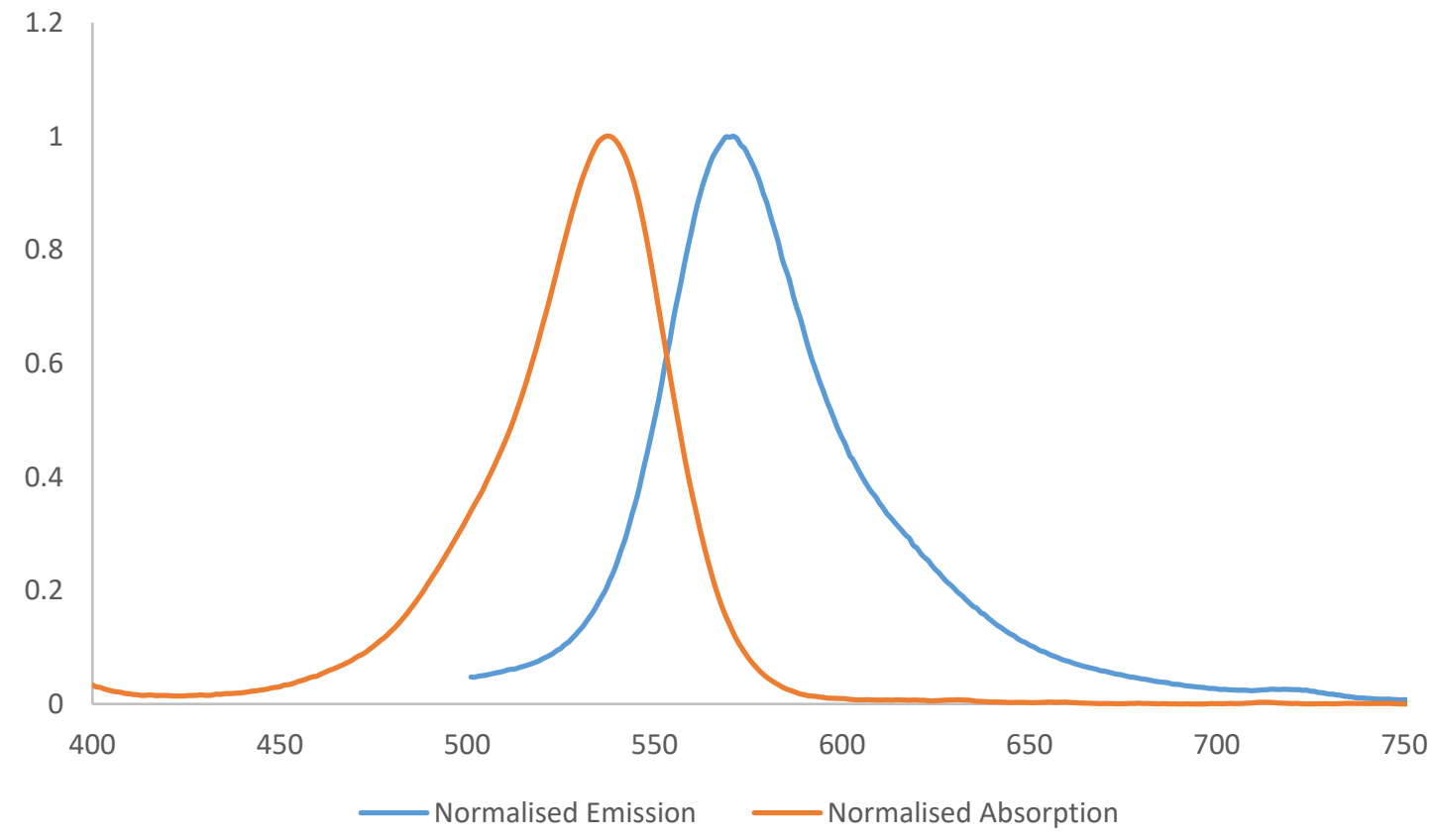

Figure S26: Normalised absorption and emission spectra of BODIPY 5 (DCM). 


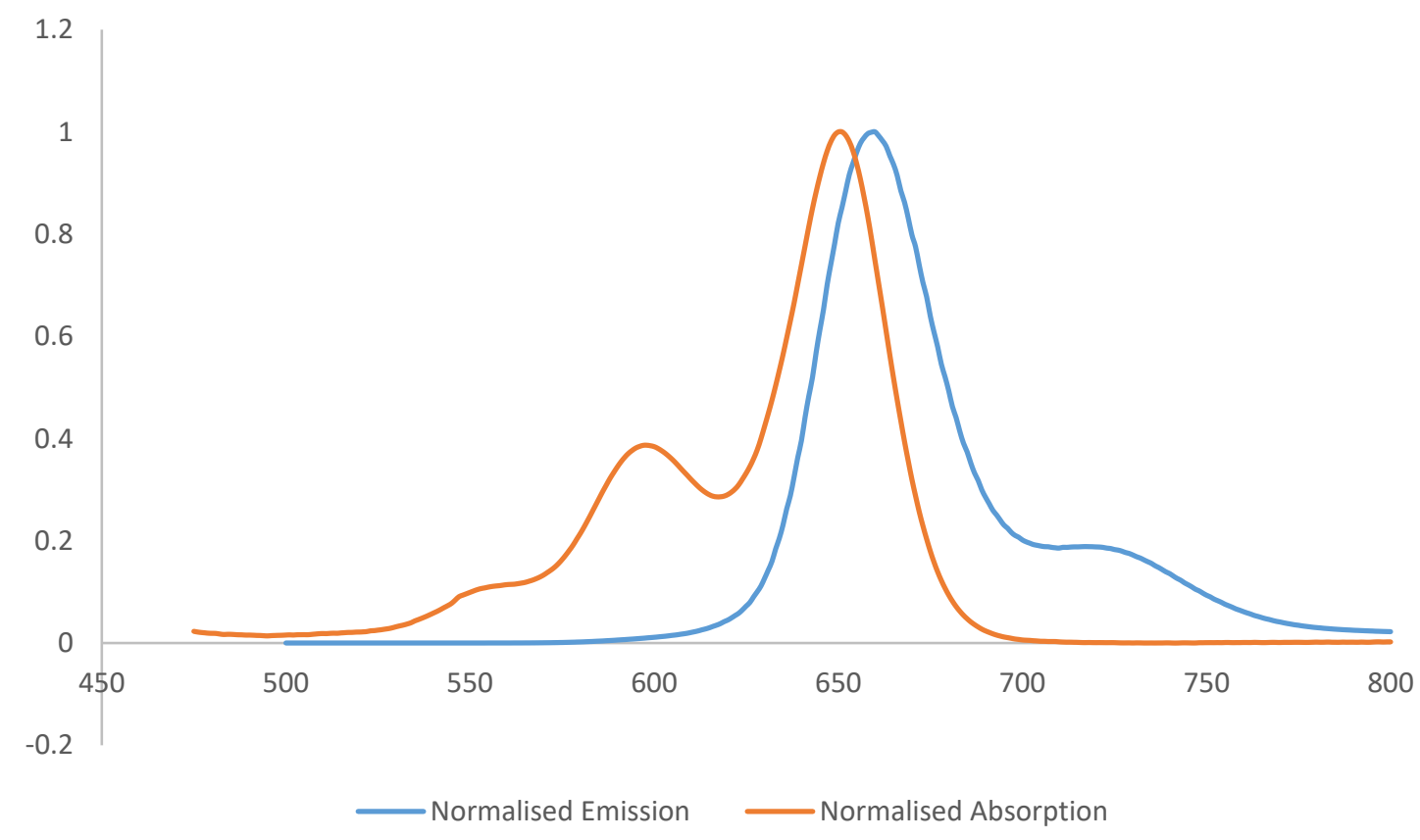

Figure S27: Normalised absorption and emission spectra of BODIPY 6 (DCM).

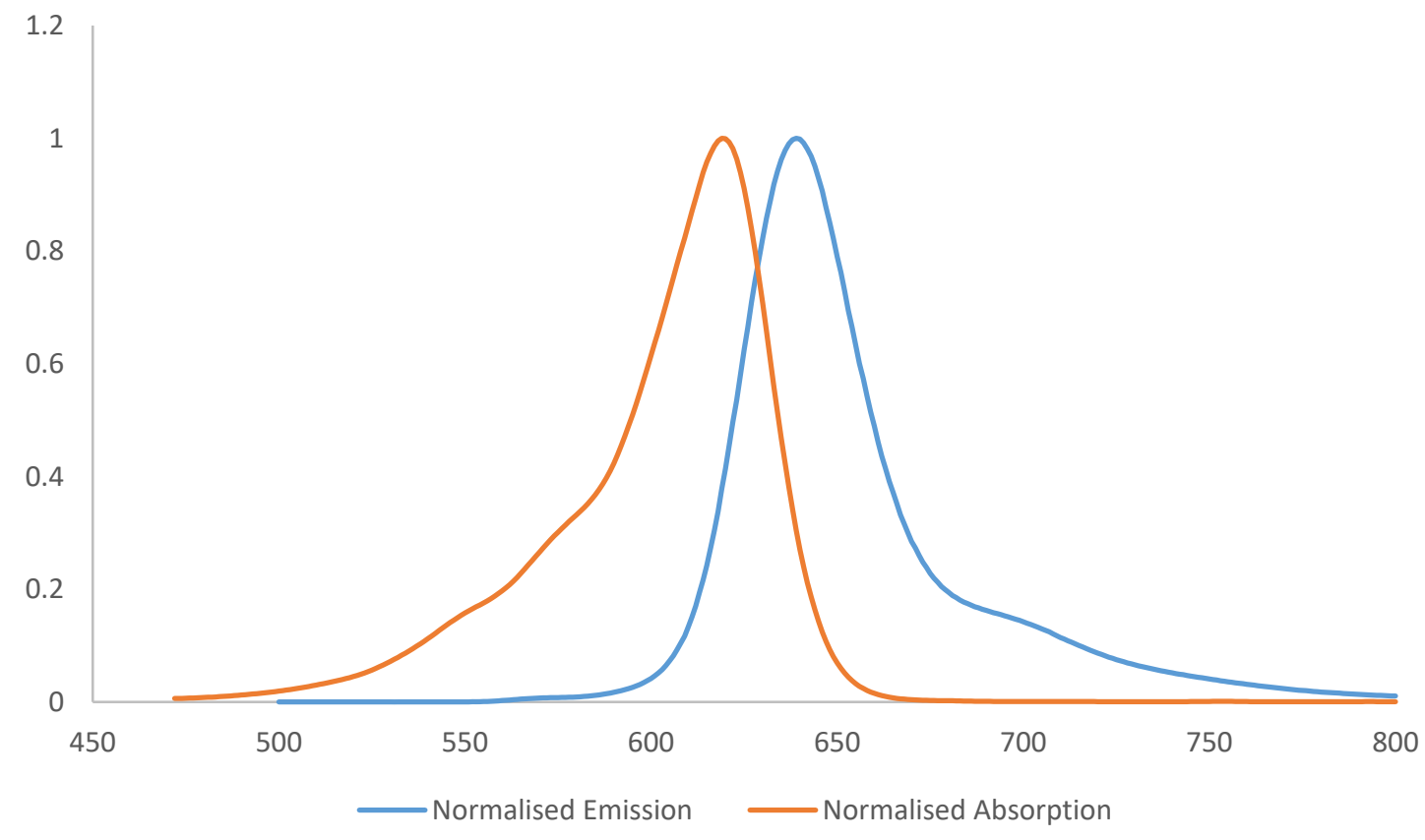

Figure S28: Normalised absorption and emission spectra of BODIPY 7 (DCM). 


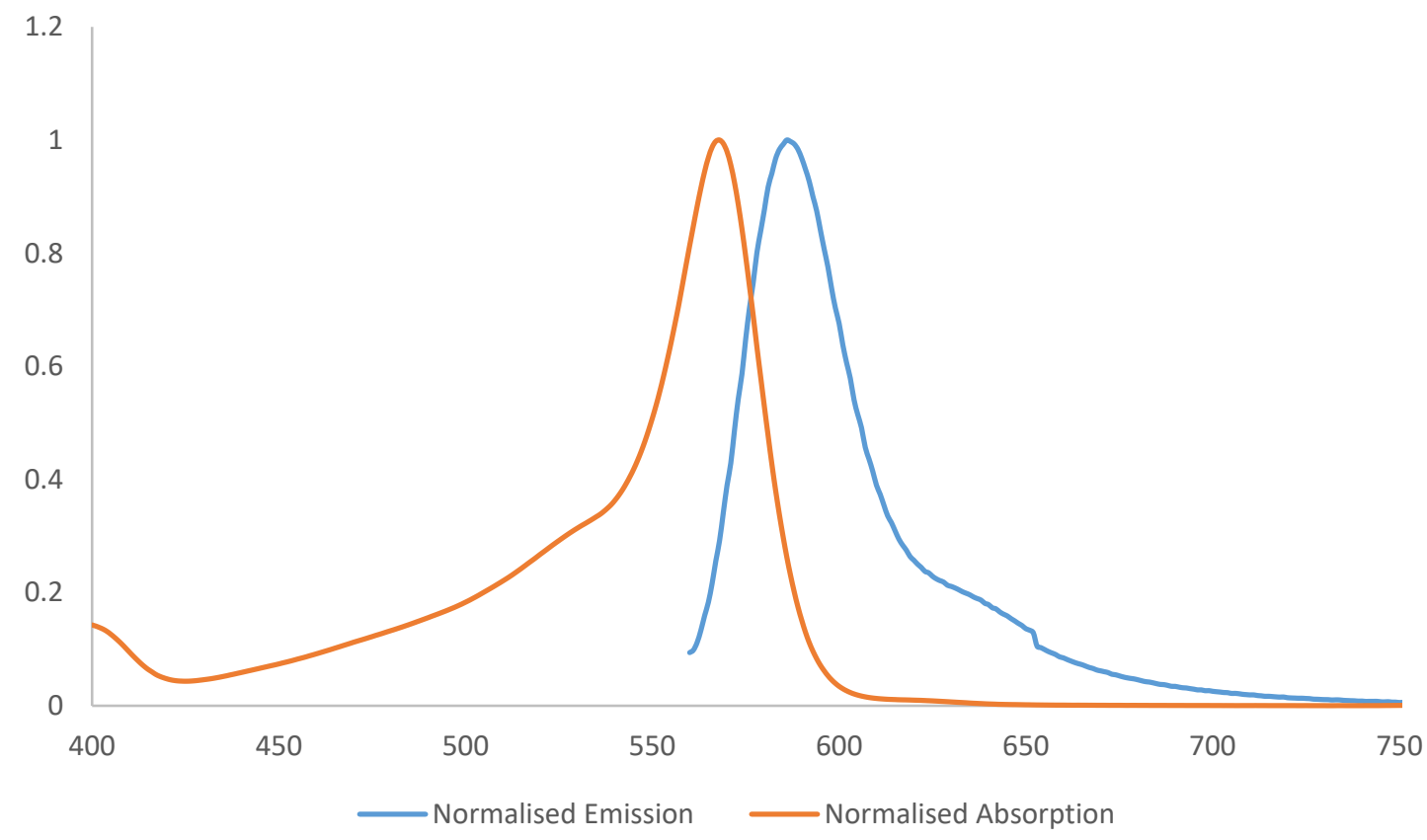

Figure S29: Normalised absorption and emission spectra of BODIPY 8 (DCM).

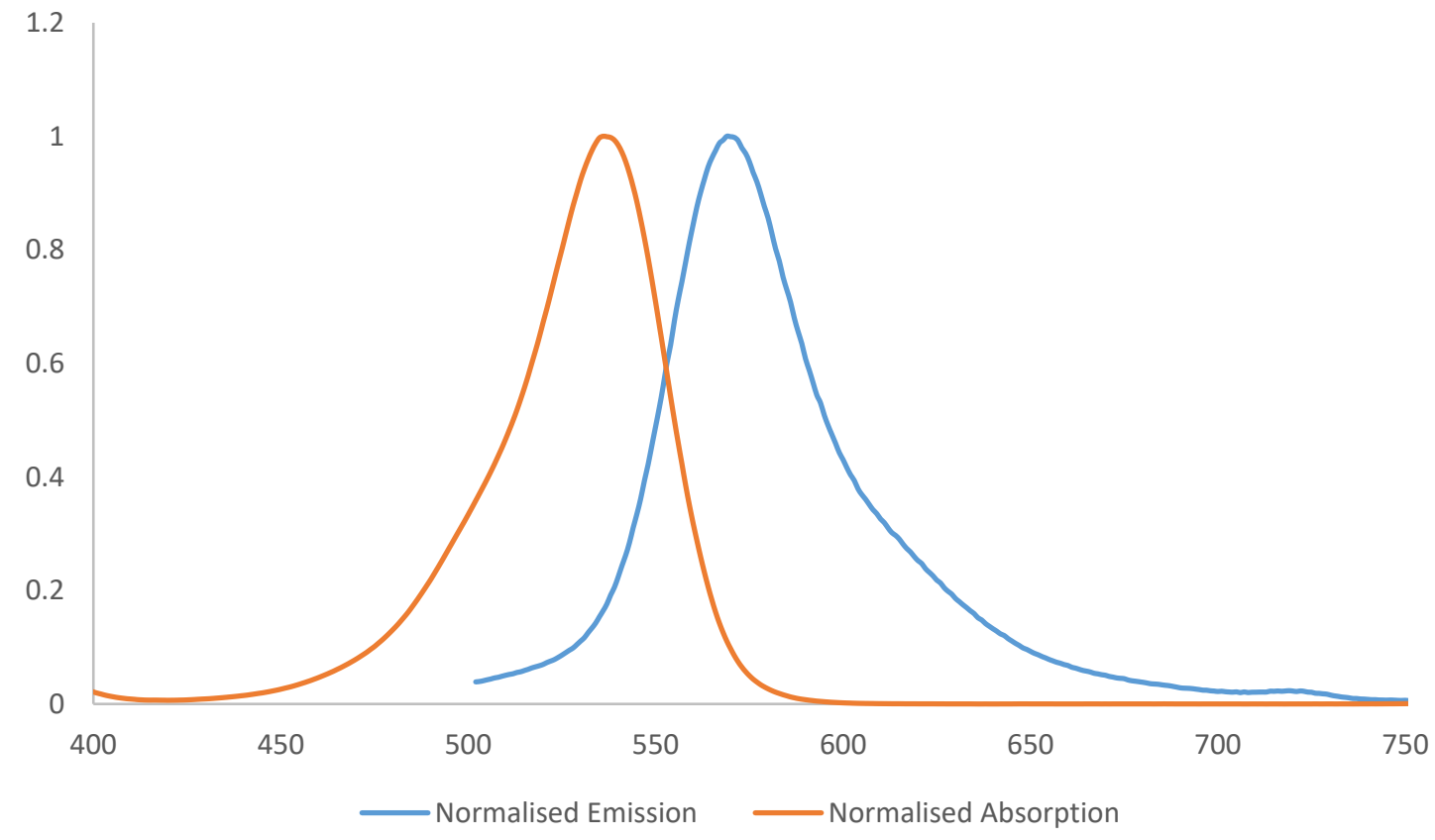

Figure S30: Normalised absorption and emission spectra of BODIPY 9 (DCM). 
Single crystal X-ray data and structure refinement

3,6-dibromo-5,5-difluoro-9-(4-methoxyphenyl)-5H-5 $\lambda^{4}$-cyclopenta[c]pyrrolo[2,1$f][1,2]$ azaborinine) (2c)

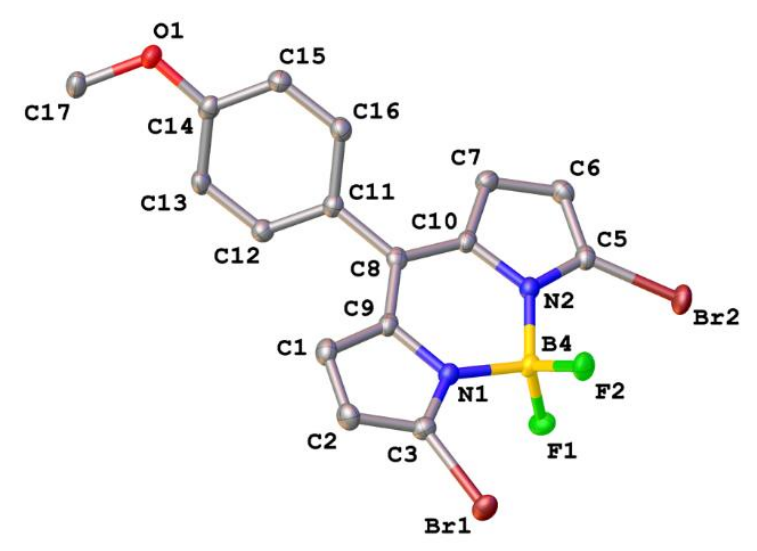

Thermal ellipsoids shown at the 50\% probability level.

Table S1 : Crystal data and structure refinement for mjh190058_fa.

Identification code

Empirical formula

Formula weight

Temperature/K

Crystal system

Space group

$\mathrm{a} / \AA$

b/

c/A

$\alpha /{ }^{\circ}$

$\beta /{ }^{\circ}$

$\gamma / 0$

Volume/ $\AA^{3}$

Z

$\rho_{\text {calc }} / \mathrm{cm}^{3}$

$\mu / \mathrm{mm}^{-1}$

$\mathrm{F}(000)$

Crystal size $/ \mathrm{mm}^{3}$ mjh190058_fa

$\mathrm{C}_{16} \mathrm{H}_{11} \mathrm{BBr}_{2} \mathrm{~F}_{2} \mathrm{~N}_{2} \mathrm{O}$

455.90

$150.0(2)$

triclinic

P-1

8.0378(3)

9.4803(4)

$11.2731(4)$

78.132(3)

83.374(3)

67.726(4)

$777.26(5)$

2

1.948

6.879

444.0

$0.39 \times 0.19 \times 0.15$ 
Radiation

$2 \Theta$ range for data collection $/^{\circ}$

Index ranges

Reflections collected

Independent reflections

Data/restraints/parameters

Goodness-of-fit on $\mathrm{F}^{2}$

Final $\mathrm{R}$ indexes $[\mathrm{I}>=2 \sigma(\mathrm{I})]$

Final $\mathrm{R}$ indexes [all data]

Largest diff. peak/hole / e $\AA^{-3}$
$\operatorname{CuK} \alpha(\lambda=1.54184)$

8.02 to 133.636

$-9 \leq \mathrm{h} \leq 8,-11 \leq \mathrm{k} \leq 11,-13 \leq 1 \leq 13$

10763

$2754\left[R_{\text {int }}=0.0185, R_{\text {sigma }}=0.0139\right]$

$2754 / 0 / 219$

1.104

$\mathrm{R}_{1}=0.0205, \mathrm{w}_{2}=0.0508$

$\mathrm{R}_{1}=0.0212, \mathrm{wR}_{2}=0.0512$

$0.51 /-0.32$

\section{Experimental}

Single crystals were grown by slow evaporation of a DCM solution.

Crystal structure data for mjh190058_fa was collected on a Xcalibur, Atlas, Gemini ultra diffractometer equipped with an fine-focus sealed X-ray tube $\left(\lambda_{\mathrm{CuK} \alpha}=1.54184 \AA\right)$ and an Oxford Cryosystems CryostreamPlus open-flow $\mathrm{N}_{2}$ cooling device. Cell refinement, data collection and data reduction were undertaken via software CrysAlisPro 1.171.38.42b (Rigaku OD, 2015). Intensities were corrected for absorption using CrysAlisPro 1.171.38.42b (Rigaku Oxford Diffraction, 2015) Analytical numeric absorption correction using a multifaceted crystal model based on expressions derived by R.C. Clark \& J.S. Reid. (Clark, R. C. \& Reid, J. S. (1995). Acta Cryst. A51, 887-897) Empirical absorption correction using spherical harmonics, implemented in SCALE3 ABSPACK scaling algorithm..

Using Olex2 [S2], the structure was solved using SHELXT 2014/5 [S3] and refined by XL [S4].

\section{Crystal structure determination of [mjh190058_fa]}

Crystal Data for $\mathrm{C}_{16} \mathrm{H}_{11} \mathrm{BBr}_{2} \mathrm{~F}_{2} \mathrm{~N}_{2} \mathrm{O}(M=455.90 \mathrm{~g} / \mathrm{mol})$ : triclinic, space group P-1 (no. 2), $a=$ 8.0378(3) $\mathrm{A}, b=9.4803(4) \AA, c=11.2731(4) \AA, \alpha=78.132(3)^{\circ}, \beta=83.374(3)^{\circ}, \gamma=$ 67.726(4) $)^{\circ}, V=777.26(5) \AA^{3}, Z=2, T=150.0(2) \mathrm{K}, \mu(\mathrm{CuK} \alpha)=6.879 \mathrm{~mm}^{-1}$, Dcalc $=$ $1.948 \mathrm{~g} / \mathrm{cm}^{3}, 10763$ reflections measured $\left(8.02^{\circ} \leq 2 \Theta \leq 133.636^{\circ}\right), 2754$ unique $\left(R_{\text {int }}=\right.$ $\left.0.0185, \mathrm{R}_{\text {sigma }}=0.0139\right)$ which were used in all calculations. The final $R_{1}$ was 0.0205 (I > $2 \sigma(\mathrm{I}))$ and $w R_{2}$ was 0.0512 (all data).

Note the crystal structure of $\mathbf{2 c}$ has been previously reported [S5]. 
3,6-dibromo-5,5-difluoro-9-(3-methoxyphenyl)-5H-5 $\lambda^{4}$-cyclopenta[c]pyrrolo[2,1$f][1,2]$ azaborinine $(2 \mathrm{~d})$

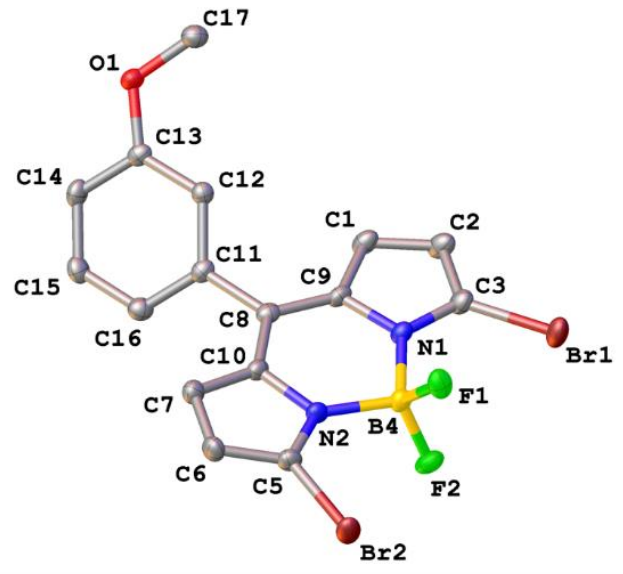

Thermal ellipsoids shown at the $50 \%$ probability level.

Table S2 : Crystal data and structure refinement for mjh190057.

Identification code

Empirical formula

Formula weight

Temperature/K

Crystal system

Space group

$\mathrm{a} / \AA$

$\mathrm{b} / \AA$

$\mathrm{c} / \AA$

$\alpha /{ }^{\circ}$

$\beta /{ }^{\circ}$

$\gamma /{ }^{\circ}$

Volume $/ \AA^{3}$

$\mathrm{Z}$

$\rho_{\text {calc }} / \mathrm{cm}^{3}$

$\mu / \mathrm{mm}^{-1}$

$\mathrm{F}(000)$

Crystal size $/ \mathrm{mm}^{3}$

Radiation

$2 \Theta$ range for data collection/ ${ }^{\circ}$ mjh190057

$\mathrm{C}_{16} \mathrm{H}_{11} \mathrm{BBr}_{2} \mathrm{~F}_{2} \mathrm{~N}_{2} \mathrm{O}$

455.90

$150.0(2)$

monoclinic

$\mathrm{P} 21 / \mathrm{c}$

8.82360(10)

$18.0320(2)$

10.39120(10)

90

108.0280(10)

90

$1572.15(3)$

4

1.926

6.802

888.0

$0.29 \times 0.18 \times 0.05$

$\mathrm{CuK \alpha}(\lambda=1.54184)$

9.81 to 133.858 
Index ranges

Reflections collected

Independent reflections

Data/restraints/parameters

Goodness-of-fit on $\mathrm{F}^{2}$

Final $\mathrm{R}$ indexes $[\mathrm{I}>=2 \sigma(\mathrm{I})]$

Final $\mathrm{R}$ indexes [all data]

Largest diff. peak/hole / e $\AA^{-3}$
$-10 \leq \mathrm{h} \leq 10,-21 \leq \mathrm{k} \leq 21,-12 \leq 1 \leq 10$

22381

$2788\left[\mathrm{R}_{\text {int }}=0.0319, \mathrm{R}_{\text {sigma }}=0.0160\right]$

$2788 / 0 / 219$

1.071

$\mathrm{R}_{1}=0.0205, \mathrm{wR}_{2}=0.0497$

$\mathrm{R}_{1}=0.0232, \mathrm{wR}_{2}=0.0514$

$0.40 /-0.45$

\section{Experimental}

Single crystals were grown by slow evaporation of a DCM solution.

Crystal structure data for mjh190057 was collected on a Xcalibur, Atlas, Gemini ultra diffractometer equipped with an fine-focus sealed X-ray tube $\left(\lambda_{\mathrm{CuK} \alpha}=1.54184 \AA\right)$ and an Oxford Cryosystems CryostreamPlus open-flow $\mathrm{N}_{2}$ cooling device. Cell refinement, data collection and data reduction were undertaken via software CrysAlisPro 1.171.38.42b (Rigaku OD, 2015). Intensities were corrected for absorption using CrysAlisPro 1.171.38.42b (Rigaku Oxford Diffraction, 2015) Empirical absorption correction using spherical harmonics, implemented in SCALE3 ABSPACK scaling algorithm..

Using Olex2 [S2], the structure was solved using SHELXT 2014/5 [S3] and refined by XL [S4].

\section{Crystal structure determination of [mjh190057]}

Crystal Data for $\mathrm{C}_{16} \mathrm{H}_{11} \mathrm{BBr}_{2} \mathrm{~F}_{2} \mathrm{~N}_{2} \mathrm{O}(M=455.90 \mathrm{~g} / \mathrm{mol})$ : monoclinic, space group $\mathrm{P} 2{ }_{1} / \mathrm{c}$ (no. 14), $a=8.82360(10) \AA, b=18.0320(2) \AA, c=10.39120(10) \AA, \beta=108.0280(10)^{\circ}, V=$ $1572.15(3) \AA^{3}, Z=4, T=150.0(2) \mathrm{K}, \mu(\mathrm{CuK \alpha})=6.802 \mathrm{~mm}^{-1}$, Dcalc $=1.926 \mathrm{~g} / \mathrm{cm}^{3}, 22381$ reflections measured $\left(9.81^{\circ} \leq 2 \Theta \leq 133.858^{\circ}\right), 2788$ unique $\left(R_{\text {int }}=0.0319, R_{\text {sigma }}=0.0160\right)$ which were used in all calculations. The final $R_{1}$ was $0.0205(\mathrm{I}>2 \sigma(\mathrm{I}))$ and $w R_{2}$ was 0.0514 (all data). 
Methyl 4-(5,5-difluoro-3,7-diiodo-5H-4 $\lambda^{4}, 5 \lambda^{4}$-dipyrrolo[1,2-c:2',1'-f][1,3,2]diazaborinin10-yl)benzoate (3a)

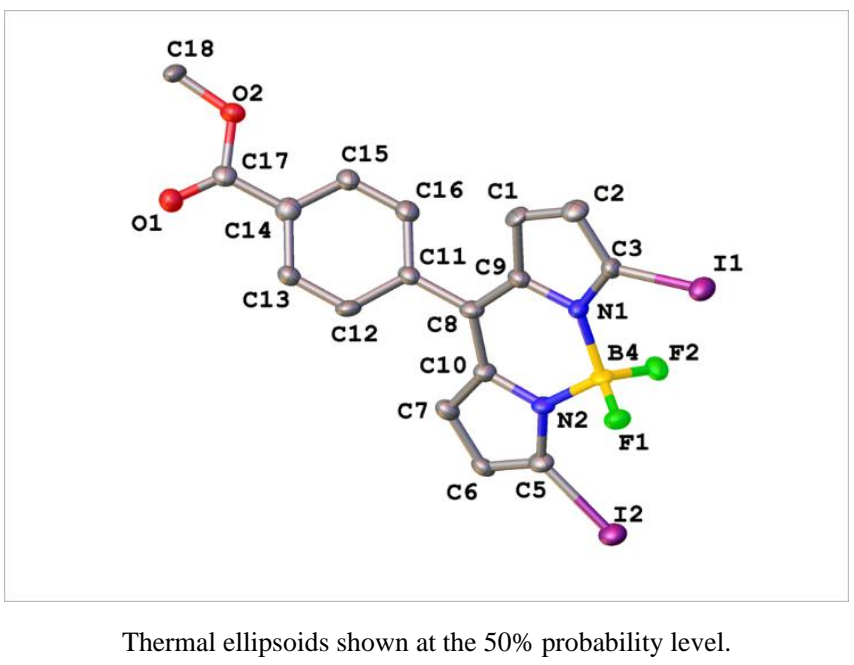

Table S3 : Crystal data and structure refinement for mjh190008_2_fa.

Identification code

Empirical formula

Formula weight

Temperature/K

Crystal system

Space group

$\mathrm{a} / \AA$

$\mathrm{b} / \AA$

$\mathrm{c} / \AA$

$\alpha /{ }^{\circ}$

$\beta /{ }^{\circ}$

$\gamma /{ }^{\circ}$

Volume $/ \AA^{3}$

Z

$\rho_{\text {calc } g / \mathrm{cm}^{3}}$

$\mu / \mathrm{mm}^{-1}$

$\mathrm{F}(000)$

Crystal size $/ \mathrm{mm}^{3}$

Radiation mjh190008_2_fa

$\mathrm{C}_{17} \mathrm{H}_{11} \mathrm{BF}_{2} \mathrm{I}_{2} \mathrm{~N}_{2} \mathrm{O}_{2}$

577.89

$150.0(2)$

triclinic

P-1

$8.0438(6)$

9.9397(9)

12.3502(12)

72.690(8)

79.848(7)

66.201(8)

$860.77(15)$

2

2.230

29.027

544.0

$0.11 \times 0.05 \times 0.01$

$\operatorname{CuK} \alpha(\lambda=1.54184)$ 
$2 \Theta$ range for data collection $/{ }^{\circ}$

Index ranges

Reflections collected

Independent reflections

Data/restraints/parameters

Goodness-of-fit on $\mathrm{F}^{2}$

Final $\mathrm{R}$ indexes $[\mathrm{I}>=2 \sigma(\mathrm{I})]$

Final $\mathrm{R}$ indexes [all data]

Largest diff. peak/hole / e $\AA^{-3}$
7.514 to 133.536

$-8 \leq \mathrm{h} \leq 9,-11 \leq \mathrm{k} \leq 11,-14 \leq 1 \leq 14$

12045

$3034\left[R_{\text {int }}=0.0760, R_{\text {sigma }}=0.0638\right]$

$3034 / 0 / 236$

1.028

$\mathrm{R}_{1}=0.0425, \mathrm{wR}_{2}=0.0980$

$\mathrm{R}_{1}=0.0616, \mathrm{wR}_{2}=0.1098$

$1.31 /-1.09$

\section{Experimental}

Single crystals were grown by slow evaporation of a DCM solution.

Crystal structure data for mjh190008_2_fa was collected on a Xcalibur, Atlas, Gemini ultra diffractometer equipped with an fine-focus sealed X-ray tube $\left(\lambda_{\mathrm{CuK} \alpha}=1.54184 \AA\right)$ and an Oxford Cryosystems CryostreamPlus open-flow $\mathrm{N}_{2}$ cooling device. Cell refinement, data collection and data reduction were undertaken via software CrysAlisPro 1.171.38.42b (Rigaku OD, 2015). Intensities were corrected for absorption using CrysAlisPro 1.171.38.42b (Rigaku Oxford Diffraction, 2015) Analytical numeric absorption correction using a multifaceted crystal model based on expressions derived by R.C. Clark \& J.S. Reid. (Clark, R. C. \& Reid, J. S. (1995). Acta Cryst. A51, 887-897) Empirical absorption correction using spherical harmonics, implemented in SCALE3 ABSPACK scaling algorithm.. Using Olex2 [S2], the structure was solved using SHELXT 2014/5 [S3] and refined by XL [S4].

\section{Crystal structure determination of [mjh190008_2_fa]}

Crystal Data for $\mathrm{C}_{17} \mathrm{H}_{11} \mathrm{BF}_{2} \mathrm{I}_{2} \mathrm{~N}_{2} \mathrm{O}_{2}(M=577.89 \mathrm{~g} / \mathrm{mol})$ : triclinic, space group P-1 (no. 2), $a=$ 8.0438(6) $\mathrm{A}, b=9.9397(9) \AA, c=12.3502(12) \AA, \alpha=72.690(8)^{\circ}, \beta=79.848(7)^{\circ}, \gamma=$ $66.201(8)^{\circ}, V=860.77(15) \AA^{3}, Z=2, T=150.0(2) \mathrm{K}, \mu(\mathrm{CuK \alpha})=29.027 \mathrm{~mm}^{-1}$, Dcalc $=$ $2.230 \mathrm{~g} / \mathrm{cm}^{3}, 12045$ reflections measured $\left(7.514^{\circ} \leq 2 \Theta \leq 133.536^{\circ}\right), 3034$ unique $\left(R_{\text {int }}=\right.$ $\left.0.0760, \mathrm{R}_{\text {sigma }}=0.0638\right)$ which were used in all calculations. The final $R_{1}$ was 0.0425 (I > $2 \sigma(\mathrm{I}))$ and $w R_{2}$ was 0.1098 (all data). 
5,5-difluoro-3,7-diiodo-10-(4-methoxyphenyl)-5H-4 $\lambda^{4}, 5 \lambda^{4}$-dipyrrolo[1,2-c:2',1'$f][1,3,2]$ diazaborinine $(3 \mathrm{c})$

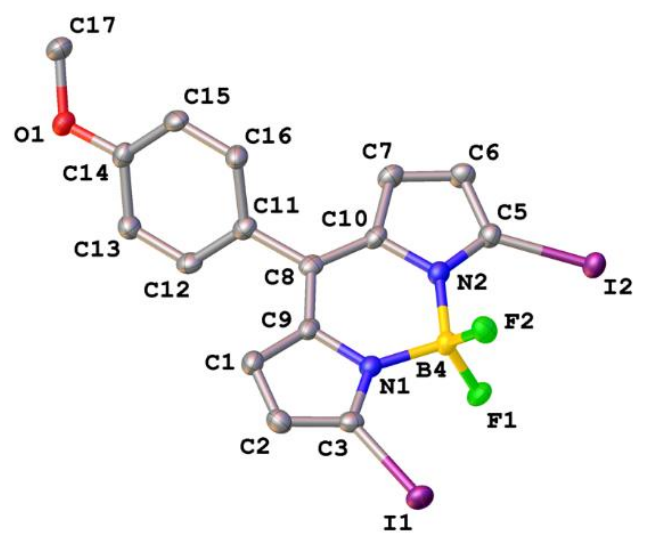

Thermal ellipsoids shown at the $50 \%$ probability level.

Table S4 : Crystal data and structure refinement for mjh190054_fa.

Identification code

Empirical formula

Formula weight

Temperature/K

Crystal system

Space group

$\mathrm{a} / \AA ̊$

$\mathrm{b} / \AA$

$\mathrm{c} / \AA$

$\alpha /{ }^{\circ}$

$\beta /{ }^{\circ}$

$\gamma /{ }^{\circ}$

Volume/ $\AA^{3}$

Z

$\rho_{\text {calc } g / \mathrm{cm}^{3}}$

$\mu / \mathrm{mm}^{-1}$

$\mathrm{F}(000)$

Crystal size $/ \mathrm{mm}^{3}$

Radiation mjh190054_fa

$\mathrm{C}_{16} \mathrm{H}_{11} \mathrm{BF}_{2} \mathrm{I}_{2} \mathrm{~N}_{2} \mathrm{O}$

549.88

$150.0(2)$

triclinic

P-1

8.1826(4)

9.9192(4)

11.3661(4)

76.970(3)

83.727(3)

66.085(4)

821.44(6)

2

2.223

30.321

516.0

$0.21 \times 0.09 \times 0.05$

$\mathrm{CuK} \alpha(\lambda=1.54184)$ 
$2 \Theta$ range for data collection $/{ }^{\circ}$

Index ranges

Reflections collected

Independent reflections

Data/restraints/parameters

Goodness-of-fit on $\mathrm{F}^{2}$

Final $\mathrm{R}$ indexes $[\mathrm{I}>=2 \sigma(\mathrm{I})]$

Final $\mathrm{R}$ indexes [all data]

Largest diff. peak/hole / e $\AA^{-3}$
7.986 to 133.746

$-9 \leq \mathrm{h} \leq 9,-11 \leq \mathrm{k} \leq 11,-13 \leq 1 \leq 13$

22214

$2895\left[R_{\text {int }}=0.0464, R_{\text {sigma }}=0.0229\right]$

$2895 / 0 / 219$

1.056

$\mathrm{R}_{1}=0.0204, \mathrm{wR}_{2}=0.0453$

$\mathrm{R}_{1}=0.0269, \mathrm{wR}_{2}=0.0482$

$0.65 /-0.47$

\section{Experimental}

Single crystals were grown by slow evaporation of a DCM solution.

Crystal structure data for mjh190054_fa was collected on a Xcalibur, Atlas, Gemini ultra diffractometer equipped with an fine-focus sealed X-ray tube $\left(\lambda_{\mathrm{CuK} \alpha}=1.54184 \AA\right)$ and an Oxford Cryosystems CryostreamPlus open-flow $\mathrm{N}_{2}$ cooling device. Cell refinement, data collection and data reduction were undertaken via software CrysAlisPro 1.171.38.42b (Rigaku OD, 2015). Intensities were corrected for absorption using CrysAlisPro 1.171.38.42b (Rigaku Oxford Diffraction, 2015) Analytical numeric absorption correction using a multifaceted crystal model based on expressions derived by R.C. Clark \& J.S. Reid. (Clark, R. C. \& Reid, J. S. (1995). Acta Cryst. A51, 887-897) Empirical absorption correction using spherical harmonics, implemented in SCALE3 ABSPACK scaling algorithm.. Using Olex2 [S2], the structure was solved using SHELXT 2014/5 [S3] and refined by XL [S4].

\section{Crystal structure determination of [mjh190054_fa]}

Crystal Data for $\mathrm{C}_{16} \mathrm{H}_{11} \mathrm{BF}_{2} \mathrm{I}_{2} \mathrm{~N}_{2} \mathrm{O}(M=549.88 \mathrm{~g} / \mathrm{mol})$ : triclinic, space group P-1 (no. 2), $a=$ 8.1826(4) $\AA, b=9.9192(4) \AA, c=11.3661(4) \AA, \alpha=76.970(3)^{\circ}, \beta=83.727(3)^{\circ}, \gamma=$ 66.085(4) $)^{\circ}, V=821.44(6) \AA^{3}, Z=2, T=150.0(2) \mathrm{K}, \mu(\mathrm{CuK \alpha})=30.321 \mathrm{~mm}^{-1}$, Dcalc $=$ $2.223 \mathrm{~g} / \mathrm{cm}^{3}, 22214$ reflections measured $\left(7.986^{\circ} \leq 2 \Theta \leq 133.746^{\circ}\right), 2895$ unique $\left(R_{\text {int }}=\right.$ $\left.0.0464, \mathrm{R}_{\text {sigma }}=0.0229\right)$ which were used in all calculations. The final $R_{1}$ was 0.0204 (I > $2 \sigma(\mathrm{I}))$ and $w R_{2}$ was 0.0482 (all data). 
5,5-difluoro-3,7-diiodo-10-(3-methoxyphenyl)-5H-4 $\lambda^{4}, 5 \lambda^{4}$-dipyrrolo[1,2-c:2',1'$f][1,3,2]$ diazaborinine (3d)

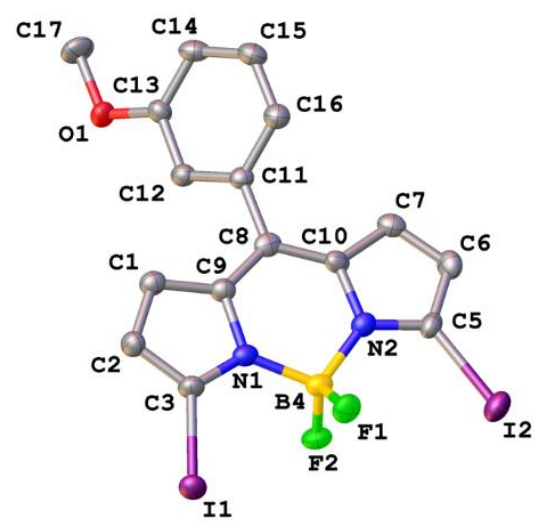

Thermal ellipsoids shown at the $50 \%$ probability level.

Table S5 : Crystal data and structure refinement for mjh190056_fa.

Identification code

Empirical formula

Formula weight

Temperature/K

Crystal system

Space group

$\mathrm{a} / \AA$

$\mathrm{b} / \AA$

$\mathrm{c} / \AA$

$\alpha /{ }^{\circ}$

$\beta /{ }^{\circ}$

$\gamma /{ }^{\circ}$

Volume $/ \AA^{3}$

Z

$\rho_{\text {calc }} \mathrm{g} / \mathrm{cm}^{3}$

$\mu / \mathrm{mm}^{-1}$

$\mathrm{F}(000)$

Crystal size $/ \mathrm{mm}^{3}$

Radiation mjh190056_fa

$\mathrm{C}_{16} \mathrm{H}_{11} \mathrm{BF}_{2} \mathrm{I}_{2} \mathrm{~N}_{2} \mathrm{O}$

549.88

$150.0(2)$

monoclinic

Ia

8.16479(11)

14.87954(18)

14.13712(16)

90

91.5292(11)

90

1716.88(4)

4

2.127

29.014

1032.0

$0.13 \times 0.11 \times 0.07$

$\mathrm{CuK} \alpha(\lambda=1.54184)$ 
$2 \Theta$ range for data collection/ ${ }^{\circ}$

Index ranges

Reflections collected

Independent reflections

Data/restraints/parameters

Goodness-of-fit on $\mathrm{F}^{2}$

Final $\mathrm{R}$ indexes $[\mathrm{I}>=2 \sigma(\mathrm{I})]$

Final $\mathrm{R}$ indexes [all data]

Largest diff. peak/hole / e $\AA^{-3}$

Flack parameter
8.63 to 133.584

$-9 \leq \mathrm{h} \leq 9,-17 \leq \mathrm{k} \leq 17,-16 \leq 1 \leq 16$

12265

$2603\left[R_{\text {int }}=0.0366, R_{\text {sigma }}=0.0289\right]$

$2603 / 2 / 219$

1.078

$\mathrm{R}_{1}=0.0308, \mathrm{wR}_{2}=0.0769$

$\mathrm{R}_{1}=0.0319, \mathrm{wR}_{2}=0.0781$

$1.01 /-0.67$

$-0.023(13)$

\section{Experimental}

Single crystals were grown by slow evaporation of a DCM solution.

Crystal structure data for mjh190056_fa was collected on a Xcalibur, Atlas, Gemini ultra diffractometer equipped with an fine-focus sealed X-ray tube $\left(\lambda_{\mathrm{CuK} \alpha}=1.54184 \AA\right)$ and an Oxford Cryosystems CryostreamPlus open-flow $\mathrm{N}_{2}$ cooling device. Cell refinement, data collection and data reduction were undertaken via software CrysAlisPro 1.171.38.42b (Rigaku OD, 2015). Intensities were corrected for absorption using CrysAlisPro 1.171.38.42b (Rigaku Oxford Diffraction, 2015) Analytical numeric absorption correction using a multifaceted crystal model based on expressions derived by R.C. Clark \& J.S. Reid. (Clark, R. C. \& Reid, J. S. (1995). Acta Cryst. A51, 887-897) Empirical absorption correction using spherical harmonics, implemented in SCALE3 ABSPACK scaling algorithm. Using Olex2 [S2], the structure was solved using SHELXT 2014/5 [S3] and refined by XL [S4].

\section{Crystal structure determination of [mjh190056_fa]}

Crystal Data for $\mathrm{C}_{16} \mathrm{H}_{11} \mathrm{BF}_{2} \mathrm{I}_{2} \mathrm{~N}_{2} \mathrm{O}(M=549.88 \mathrm{~g} / \mathrm{mol})$ : monoclinic, space group Ia (no. 9), $a=$ 8.16479(11) $\AA, b=14.87954(18) \AA, c=14.13712(16) \AA, \beta=91.5292(11)^{\circ}, V=$ 1716.88(4) $\AA^{3}, Z=4, T=150.0(2) \mathrm{K}, \mu(\mathrm{CuK \alpha})=29.014 \mathrm{~mm}^{-1}$, Dcalc $=2.127 \mathrm{~g} / \mathrm{cm}^{3}, 12265$ reflections measured $\left(8.63^{\circ} \leq 2 \Theta \leq 133.584^{\circ}\right), 2603$ unique $\left(R_{\text {int }}=0.0366, R_{\text {sigma }}=0.0289\right)$ which were used in all calculations. The final $R_{1}$ was 0.0308 (I $\left.>2 \sigma(\mathrm{I})\right)$ and $w R_{2}$ was 0.0781 (all data). 
Methyl 4-(5,5-difluoro-3,7-diphenyl-5H-4 $\lambda^{4}, 5 \lambda^{4}$-dipyrrolo[1,2-c:2',1'-

$f][1,3,2]$ diazaborinin-10-yl)benzoate (4)

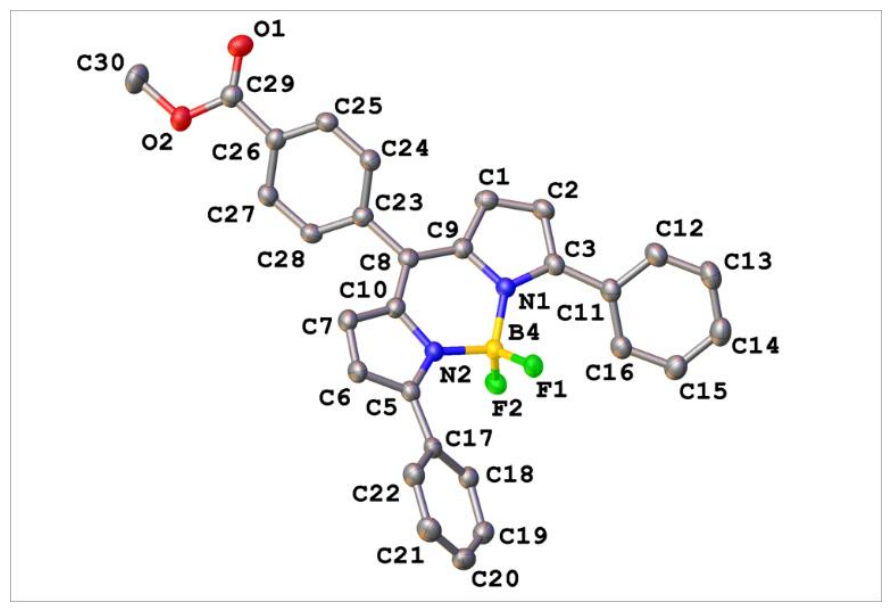

Thermal ellipsoids shown at the $50 \%$ probability level.

Table S6 : Crystal data and structure refinement for mjh210013.

Identification code

Empirical formula

Formula weight

Temperature/K

Crystal system

Space group

$\mathrm{a} / \AA ̊$

$\mathrm{b} / \AA$

c/Å

$\alpha /{ }^{\circ}$

$\beta /{ }^{\circ}$

$\gamma /{ }^{\circ}$

Volume $/ \AA^{3}$

Z

$\rho_{\text {calc } g / \mathrm{cm}^{3}}$

$\mu / \mathrm{mm}^{-1}$

$\mathrm{F}(000)$

Crystal size $/ \mathrm{mm}^{3}$

Radiation mjh210013

$\mathrm{C}_{29} \mathrm{H}_{21} \mathrm{BF}_{2} \mathrm{~N}_{2} \mathrm{O}_{2}$

478.29

150.0(2)

triclinic

P-1

7.0297(2)

12.3907(4)

13.2309(4)

$86.775(2)$

86.861(2)

87.872(2)

1148.23(6)

2

1.383

0.803

496.0

$0.29 \times 0.22 \times 0.1$

$\mathrm{CuK} \alpha(\lambda=1.54184)$ 
$2 \Theta$ range for data collection/ ${ }^{\circ} 7.15$ to 133.786

Index ranges

Reflections collected

Independent reflections

Data/restraints/parameters

Goodness-of-fit on $\mathrm{F}^{2}$

Final $R$ indexes $[\mathrm{I}>=2 \sigma(\mathrm{I})]$

Final $\mathrm{R}$ indexes [all data]

Largest diff. peak/hole / e $\AA^{-3} 0.23 /-0.16$
$-8 \leq \mathrm{h} \leq 8,-14 \leq \mathrm{k} \leq 14,-15 \leq 1 \leq 15$

30645

$4056\left[\mathrm{R}_{\text {int }}=0.0511, \mathrm{R}_{\text {sigma }}=0.0218\right]$

$4056 / 0 / 327$

1.031

$\mathrm{R}_{1}=0.0364, \mathrm{wR}_{2}=0.0950$

$\mathrm{R}_{1}=0.0419, \mathrm{wR}_{2}=0.1006$

\section{Experimental}

Single crystals were grown by slow evaporation of a DCM solution.

Crystal structure data for mjh210013 was collected on a Xcalibur, Atlas, Gemini ultra diffractometer equipped with an fine-focus sealed X-ray tube $\left(\lambda_{\mathrm{Cu} \mathrm{K} \alpha}=1.54184 \AA\right)$ and an Oxford Cryosystems CryostreamPlus open-flow $\mathrm{N}_{2}$ cooling device. Cell refinement, data collection and data reduction were undertaken via software CrysAlisPro 1.171.40.82a (Rigaku OD, 2020). Intensities were corrected for absorption using CrysAlisPro 1.171.40.82a (Rigaku Oxford Diffraction, 2020) Empirical absorption correction using spherical harmonics, implemented in SCALE3 ABSPACK scaling algorithm..

Using Olex2 [S2], the structure was solved using SHELXT 2014/5 [S3] and refined by XL [S4].

\section{Crystal structure determination of [mjh210013]}

Crystal Data for $\mathrm{C}_{2} \mathrm{H}_{21} \mathrm{BF}_{2} \mathrm{~N}_{2} \mathrm{O}_{2}(M=478.29 \mathrm{~g} / \mathrm{mol})$ : triclinic, space group P-1 (no. 2), $a=$ 7.0297(2) $\mathrm{A}, b=12.3907(4) \AA, c=13.2309(4) \AA, \alpha=86.775(2)^{\circ}, \beta=86.861(2)^{\circ}, \gamma=$ 87.872(2) $)^{\circ}, V=1148.23(6) \AA^{3}, Z=2, T=150.0(2) \mathrm{K}, \mu(\mathrm{Cu} \mathrm{K \alpha})=0.803 \mathrm{~mm}^{-1}$, Dcalc $=$ $1.383 \mathrm{~g} / \mathrm{cm}^{3}, 30645$ reflections measured $\left(7.15^{\circ} \leq 2 \Theta \leq 133.786^{\circ}\right), 4056$ unique $\left(R_{\text {int }}=\right.$ $\left.0.0511, R_{\text {sigma }}=0.0218\right)$ which were used in all calculations. The final $R_{1}$ was 0.0364 (I > $2 \sigma(\mathrm{I}))$ and $w R_{2}$ was 0.1006 (all data).Thermal ellipsoids shown at the $50 \%$ probability level. 
$f][1,3,2]$ diazaborinin-10-yl)benzoate (6)

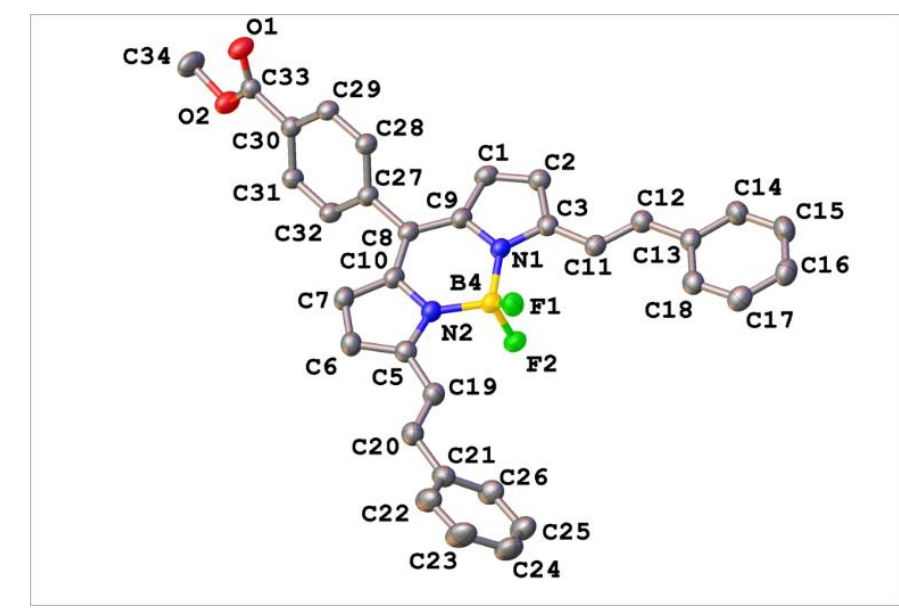

Thermal ellipsoids shown at the $50 \%$ probability level.

Table S7 : Crystal data and structure refinement for mjh200073.

Identification code

Empirical formula

Formula weight

Temperature/K

Crystal system

Space group

$\mathrm{a} / \AA$

$\mathrm{b} / \AA$

$\mathrm{c} / \AA$

$\alpha /{ }^{\circ}$

$\beta /{ }^{\circ}$

$\gamma /{ }^{\circ}$

Volume $/ \AA^{3}$

Z

$\rho_{\text {calc } g / \mathrm{cm}^{3}}$

$\mu / \mathrm{mm}^{-1}$

$\mathrm{F}(000)$

Crystal size $/ \mathrm{mm}^{3}$

Radiation mjh200073

$\mathrm{C}_{33} \mathrm{H}_{25} \mathrm{BF}_{2} \mathrm{~N}_{2} \mathrm{O}_{2}$

530.36

$150.0(2)$

monoclinic

$\mathrm{P} 21 / \mathrm{n}$

7.8877(2)

17.3177(3)

19.2110(4)

90

97.202(2)

90

2603.46(10)

4

1.353

0.764

1104.0

$0.21 \times 0.15 \times 0.06$

$\mathrm{CuK} \alpha(\lambda=1.54184)$ 
$2 \Theta$ range for data collection $/{ }^{\circ} 6.896$ to 133.622

Index ranges

Reflections collected

Independent reflections

Data/restraints/parameters

Goodness-of-fit on $\mathrm{F}^{2}$

Final $R$ indexes $[\mathrm{I}>=2 \sigma(\mathrm{I})]$

Final $\mathrm{R}$ indexes [all data]

Largest diff. peak/hole / e $\AA^{-3} 0.30 /-0.21$
$-9 \leq \mathrm{h} \leq 7,-20 \leq \mathrm{k} \leq 20,-22 \leq 1 \leq 22$

36358

$4602\left[\mathrm{R}_{\text {int }}=0.0597, \mathrm{R}_{\text {sigma }}=0.0277\right]$

$4602 / 0 / 362$

1.048

$\mathrm{R}_{1}=0.0435, \mathrm{wR}_{2}=0.1109$

$\mathrm{R}_{1}=0.0531, \mathrm{wR}_{2}=0.1207$

\section{Experimental}

Single crystals were grown by slow evaporation of a DCM solution.

Crystal structure data for mjh200073 was collected on a Xcalibur, Atlas, Gemini ultra diffractometer equipped with an fine-focus sealed X-ray tube $\left(\lambda_{\mathrm{Cu} \mathrm{K} \alpha}=1.54184 \AA\right)$ and an Oxford Cryosystems CryostreamPlus open-flow $\mathrm{N}_{2}$ cooling device. Cell refinement, data collection and data reduction were undertaken via software CrysAlisPro 1.171.40.82a (Rigaku OD, 2020). Intensities were corrected for absorption using CrysAlisPro 1.171.40.82a (Rigaku Oxford Diffraction, 2020) Empirical absorption correction using spherical harmonics, implemented in SCALE3 ABSPACK scaling algorithm.. Using Olex2 [S2], the structure was solved using SHELXT 2014/5 [S3] and refined by XL [S4].

\section{Crystal structure determination of [mjh200073]}

Crystal Data for $\mathrm{C}_{33} \mathrm{H}_{25} \mathrm{BF}_{2} \mathrm{~N}_{2} \mathrm{O}_{2}(M=530.36 \mathrm{~g} / \mathrm{mol})$ : monoclinic, space group $\mathrm{P} 21 / \mathrm{n}$ (no. 14), $a=7.8877(2) \AA, b=17.3177(3) \AA, c=19.2110(4) \AA, \beta=97.202(2)^{\circ}, V=$ 2603.46(10) $\AA^{3}, Z=4, T=150.0(2) \mathrm{K}, \mu(\mathrm{Cu} \mathrm{K \alpha})=0.764 \mathrm{~mm}^{-1}$, Dcalc $=1.353 \mathrm{~g} / \mathrm{cm}^{3}, 36358$ reflections measured $\left(6.896^{\circ} \leq 2 \Theta \leq 133.622^{\circ}\right), 4602$ unique $\left(R_{\text {int }}=0.0597, R_{\text {sigma }}=0.0277\right)$ which were used in all calculations. The final $R_{1}$ was $0.0435(\mathrm{I}>2 \sigma(\mathrm{I}))$ and $w R_{2}$ was 0.1207 (all data). 


\section{References}

S1. Frank F., Manzoli L.A., Mauker P., Alsimaree A.A., Waddell P.G., Probert M.R., Penfold T.J., Knight J.G., Hall M.J. Tetrahedron 2020, 76(17), 131113.

S2. Dolomanov, O.V., Bourhis, L.J., Gildea, R.J, Howard, J.A.K. \& Puschmann, H. J. Appl. Cryst. 2009, 42, 339.

S3. Sheldrick, G.M. Acta Cryst. 2015, A71, 3-8.

S4. Sheldrick, G.M. Acta Cryst. 2008, A64, 112-122.

S5. $\quad$ Lakshmi, V., Ravikanth, M. Dalton Trans. 2012, 41, 5903-5911. 
${ }^{1}$ H NMR (300 MHz, CDCl 3 ): Methyl 4-(5,5-difluoro-3,7-diiodo-5H-4 $\lambda^{4}, 5 \lambda^{4}$-dipyrrolo[1,2-c:2',1'-f][1,3,2]diazaborinin-10-yl)benzoate (3a)

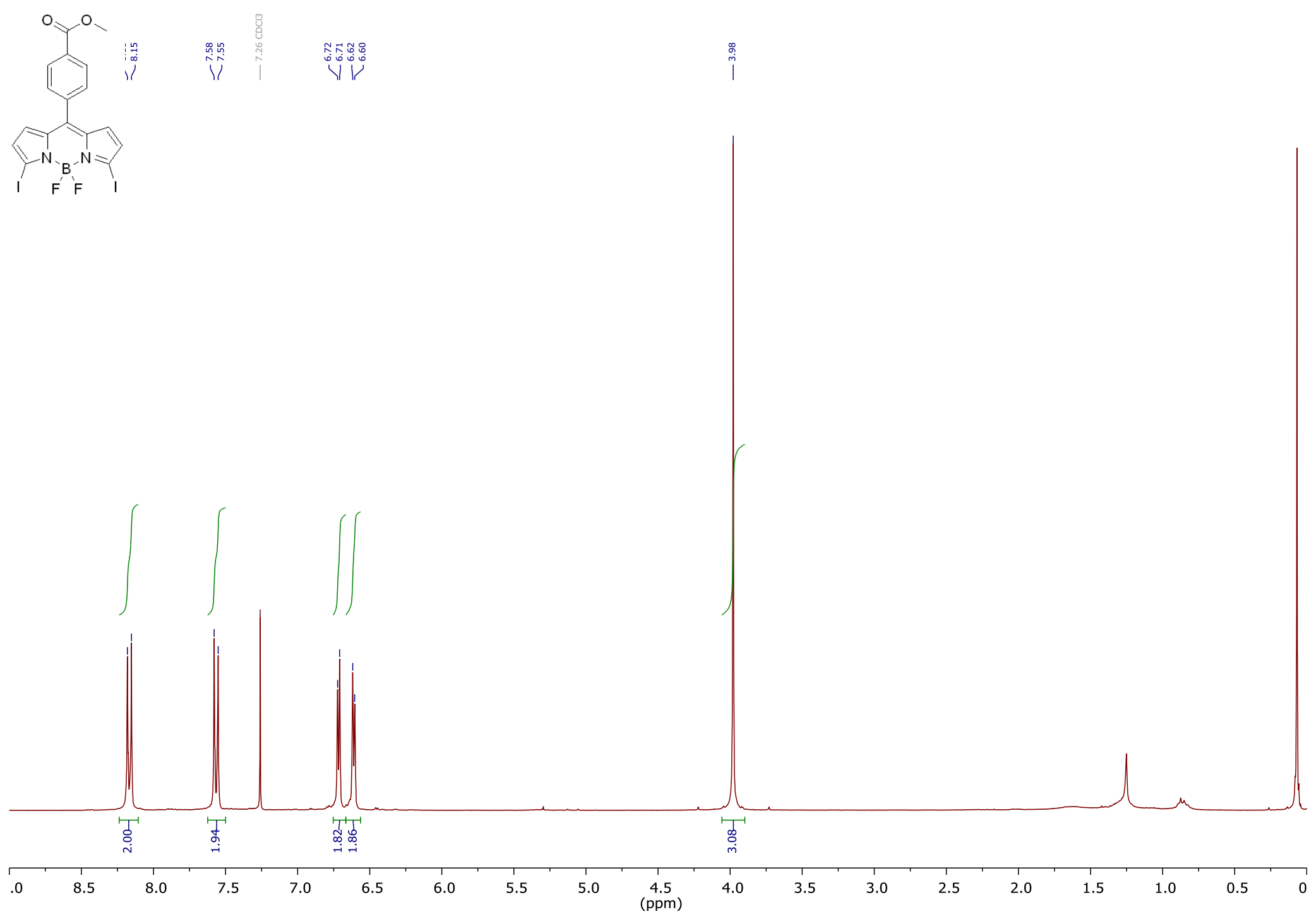


${ }^{13} \mathrm{C}$ NMR (75 MHz, CDCl 3$)$ : Methyl 4-(5,5-difluoro-3,7-diiodo-5 $H-4 \lambda^{4}, 5 \lambda^{4}$-dipyrrolo[1,2-c:2',1'-f][1,3,2]diazaborinin-10-yl)benzoate (3a)
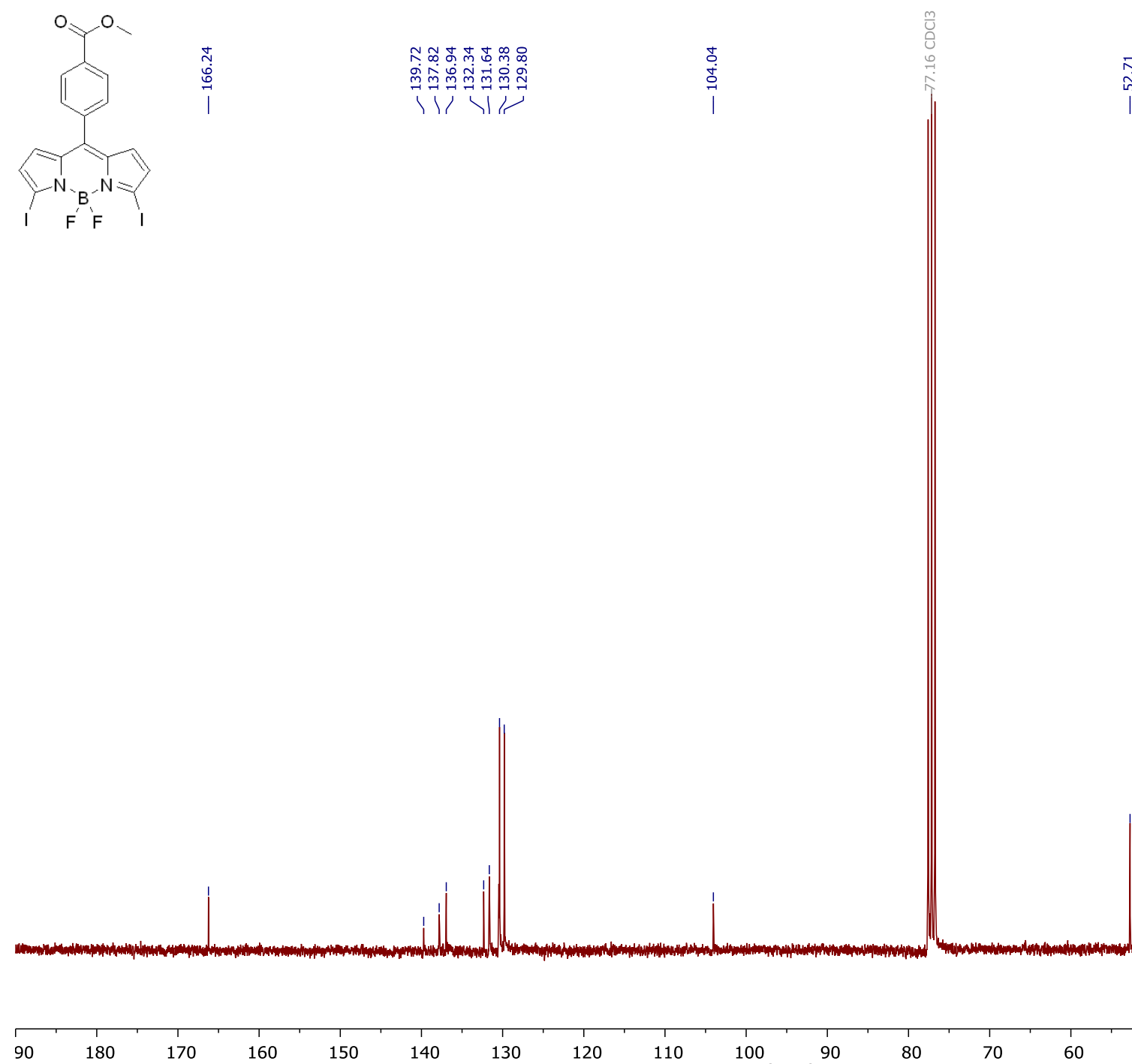

180

$170 \quad 160$
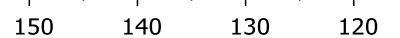

110 $00(\mathrm{ppm}){ }^{90}$ 
${ }^{19}$ F NMR (282 MHz, CDCl 3 ): Methyl 4-(5,5-difluoro-3,7-diiodo-5H-4 $\lambda^{4}, 5 \lambda^{4}$-dipyrrolo[1,2-c:2',1'-f][1,3,2]diazaborinin-10-yl)benzoate (3a)

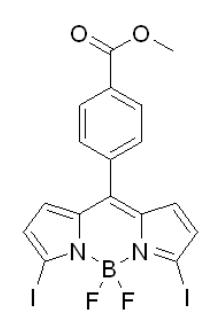

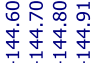

iii

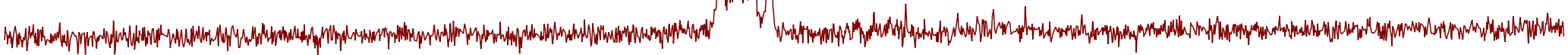

$\begin{array}{llllllllllllllllllll}-140.5 & -141.0 & -141.5 & -142.0 & -142.5 & -143.0 & -143.5 & -144.0 & -144.5 & -145.0 & -145.5 & -146.0 & -146.5 & -147.0 & -147.5 & -148.0 & -148.5 & -149.0 & -149.5 & -15\end{array}$ 
${ }^{11}$ B NMR (96 MHz, CDCl $)$ : Methyl 4-(5,5-difluoro-3,7-diiodo-5H-4 $\lambda^{4}, 5 \lambda^{4}$-dipyrrolo[1,2-c:2',1'-f][1,3,2]diazaborinin-10-yl)benzoate (3a)

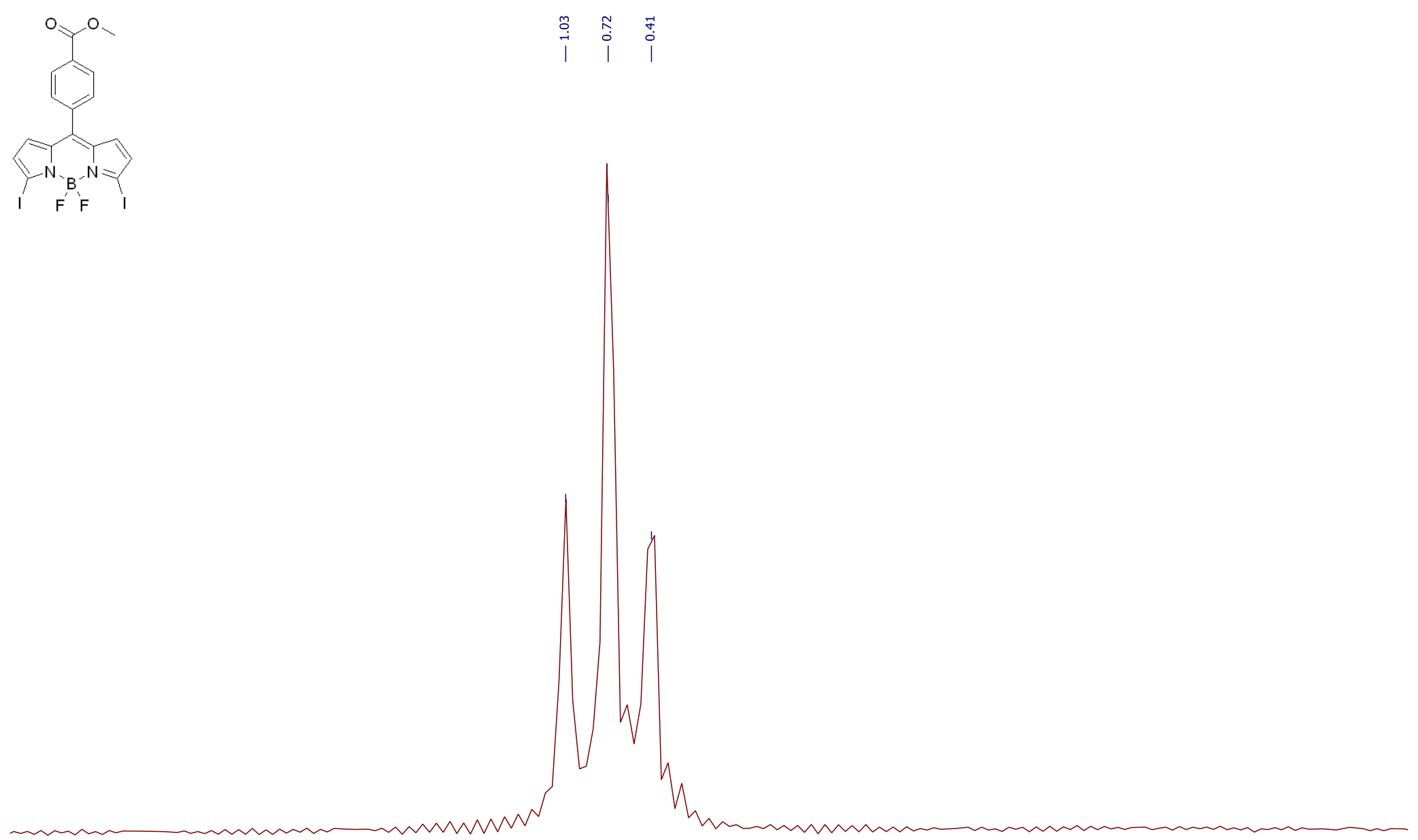

$\begin{array}{lllllll}4.5 & 4.0 & 3.5 & 3.0 & 2.5 & 2.0 & 1.5\end{array}$ 
${ }^{1} \mathrm{H}$ NMR (300 MHz, CDCl 3 ): 5,5-difluoro-3,7-diiodo-10-(3-nitrophenyl)-5H-4 $\lambda^{4}, 5 \lambda^{4}$-dipyrrolo[1,2-c:2',1'-f][1,3,2]diazaborinine (3b)
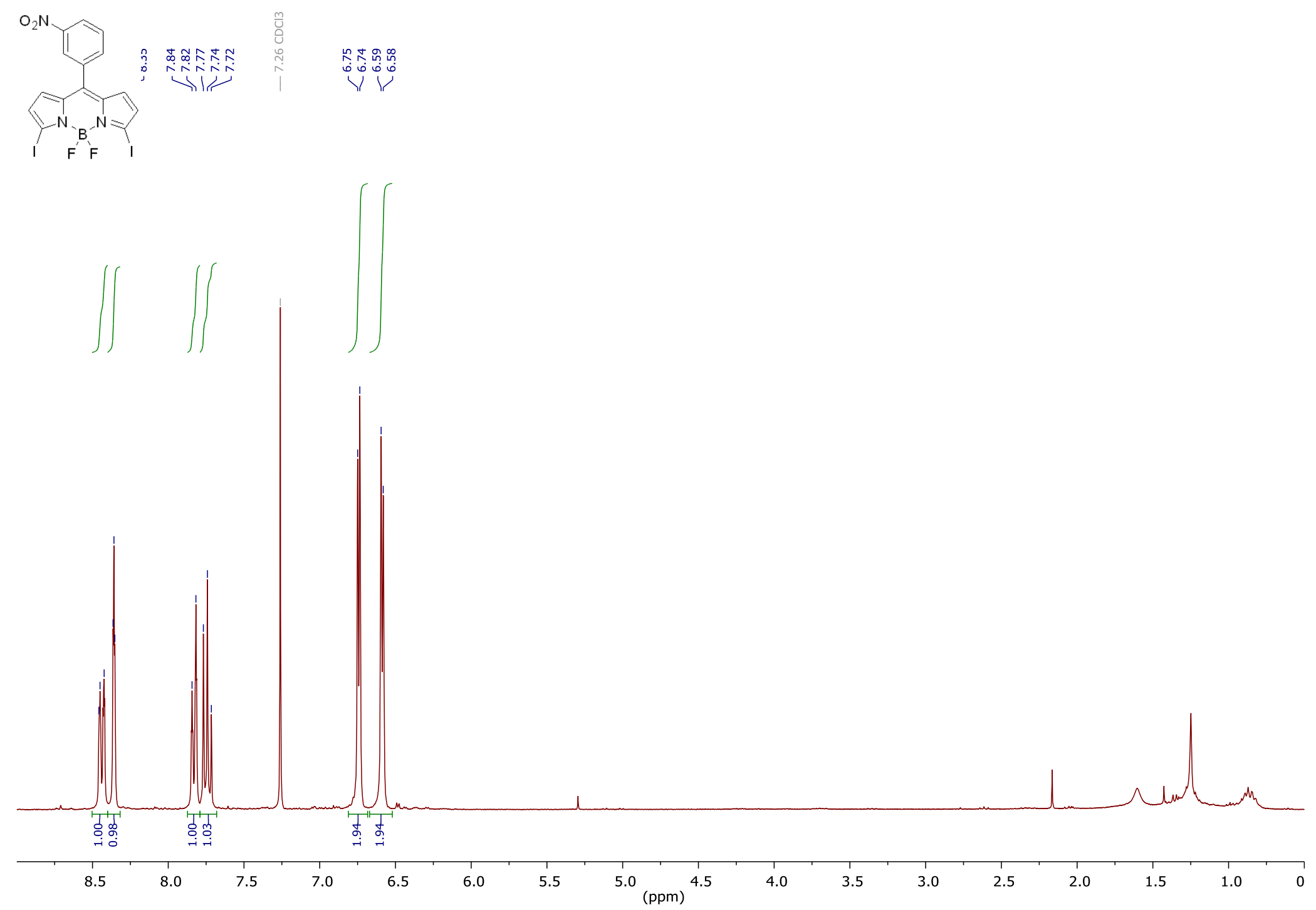
${ }^{13} \mathrm{C}$ NMR (75 MHz, CDCl 3$)$ : 5,5-difluoro-3,7-diiodo-10-(3-nitrophenyl)-5H-4 $\lambda^{4}, 5 \lambda^{4}$-dipyrrolo[1,2-c:2',1'-f][1,3,2]diazaborinine (3b)
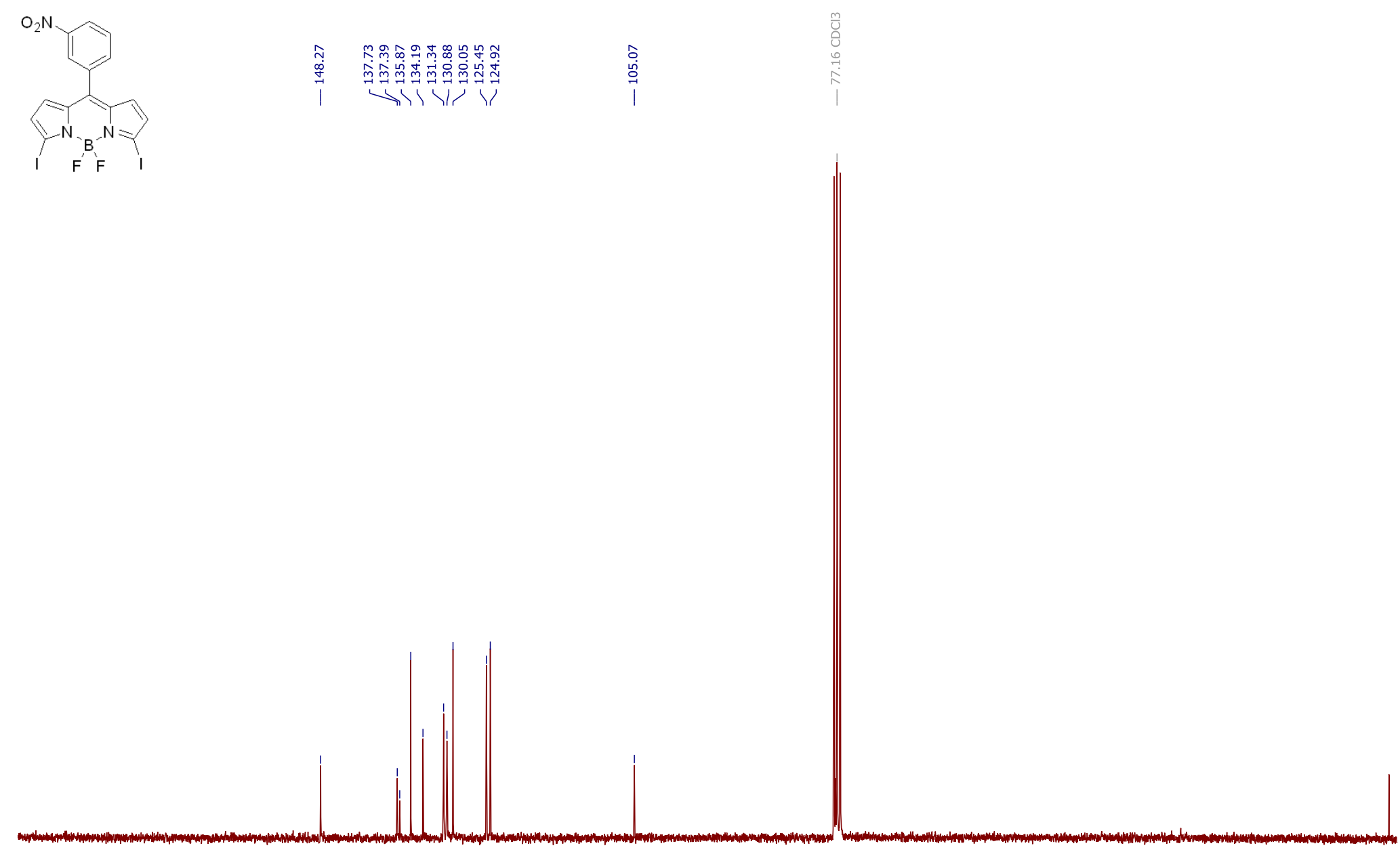

$$
90
$$

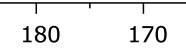

160

150

140
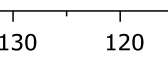
${ }^{19}$ F NMR (282 MHz, CDCl 3$):$ 5,5-difluoro-3,7-diiodo-10-(3-nitrophenyl)-5H-4 $\lambda^{4}, 5 \lambda^{4}$-dipyrrolo[1,2-c:2',1'-f][1,3,2]diazaborinine (3b)

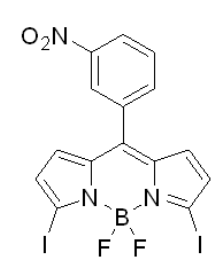

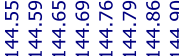

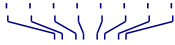

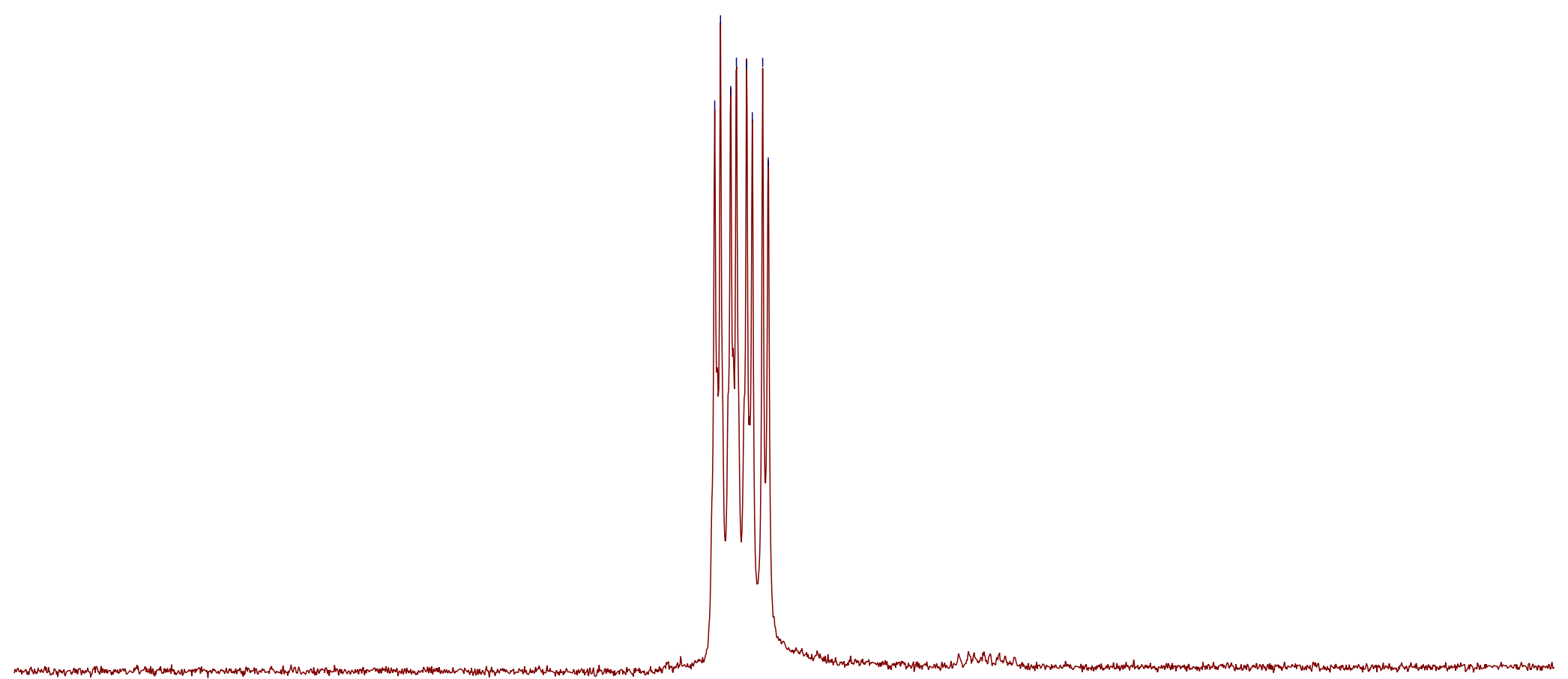

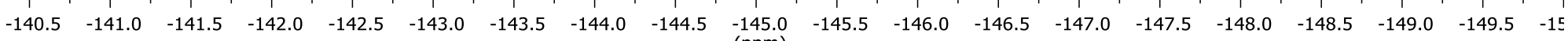


${ }^{11}$ B NMR (96 MHz, CDCl 3 ): 5,5-difluoro-3,7-diiodo-10-(3-nitrophenyl)-5H-4 $\lambda^{4}, 5 \lambda^{4}$-dipyrrolo[1,2-c:2',1'-f][1,3,2] diazaborinine (3b)
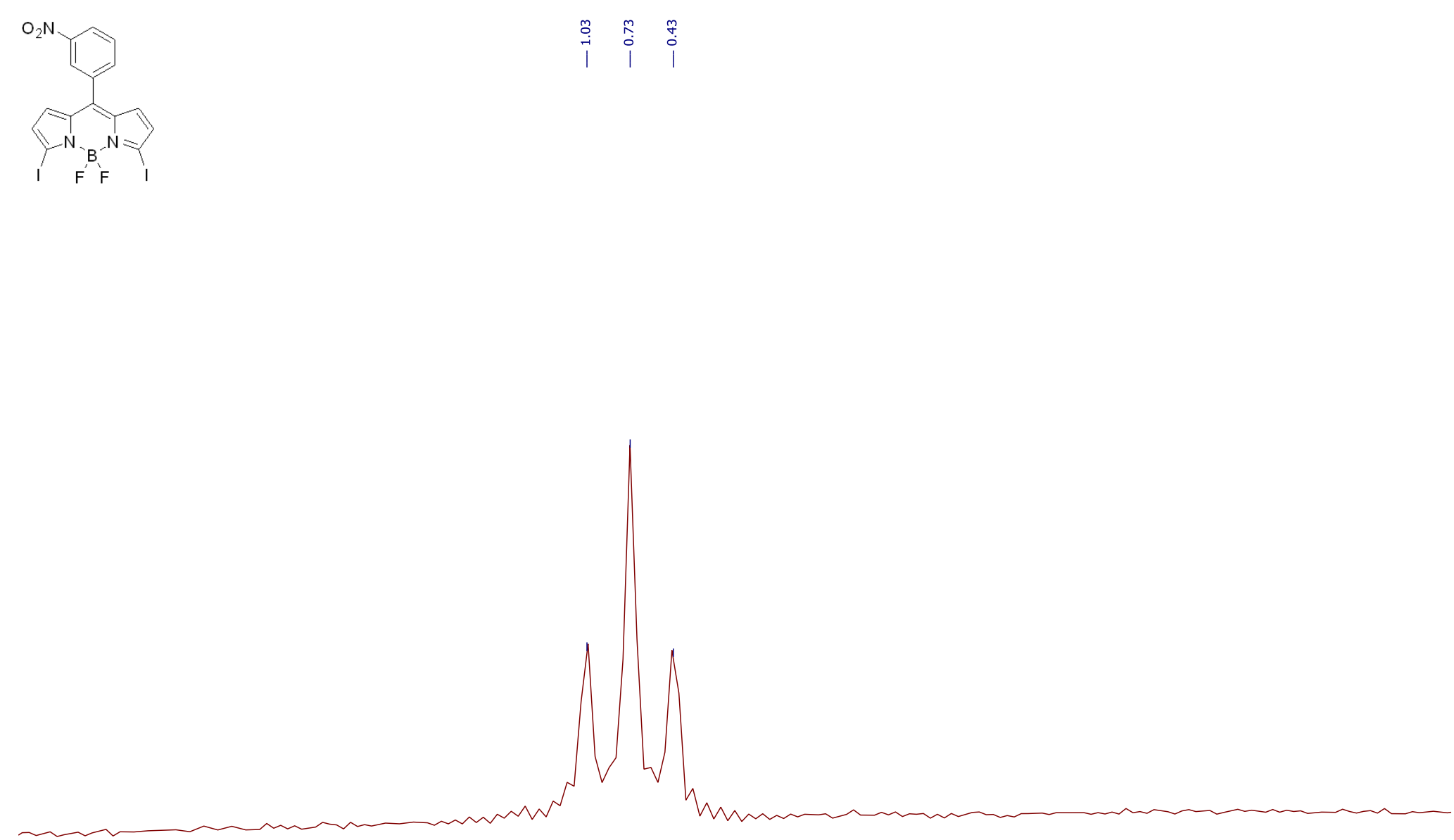
${ }^{1}$ H NMR (300 MHz, CDCl 3$)$ : 5,5-difluoro-3,7-diiodo-10-(4-methoxyphenyl)-5H-4 $\lambda^{4}, 5 \lambda^{4}$-dipyrrolo[1,2-c:2',1'-f][1,3,2]diazaborinine (3c)

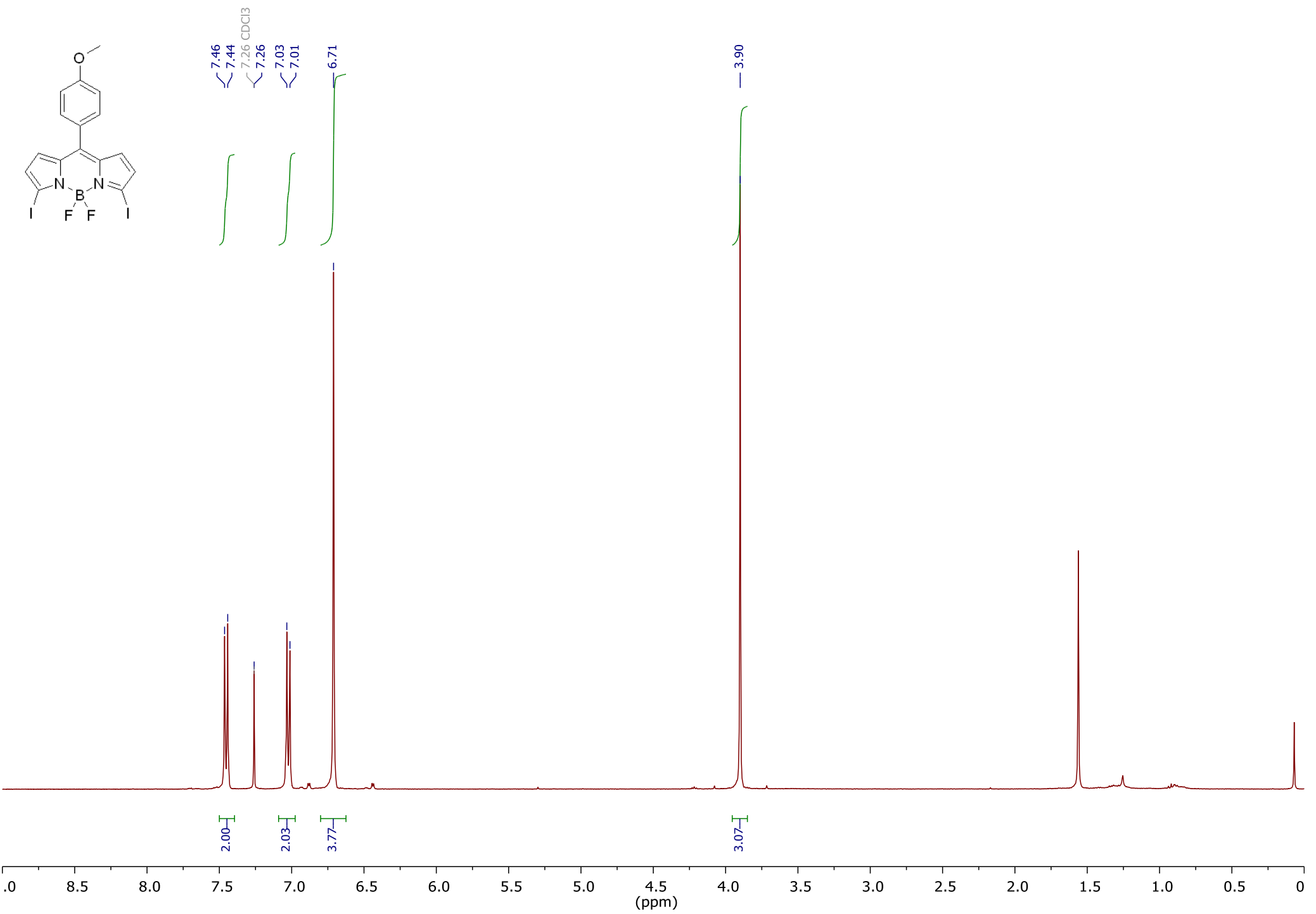


${ }^{13} \mathrm{C}$ NMR (75 MHz, CDCl 3 ): 5,5-difluoro-3,7-diiodo-10-(4-methoxyphenyl)-5H-4 $\lambda^{4}, 5 \lambda^{4}$-dipyrrolo[1,2-c:2',1'-f][1,3,2]diazaborinine (3c)
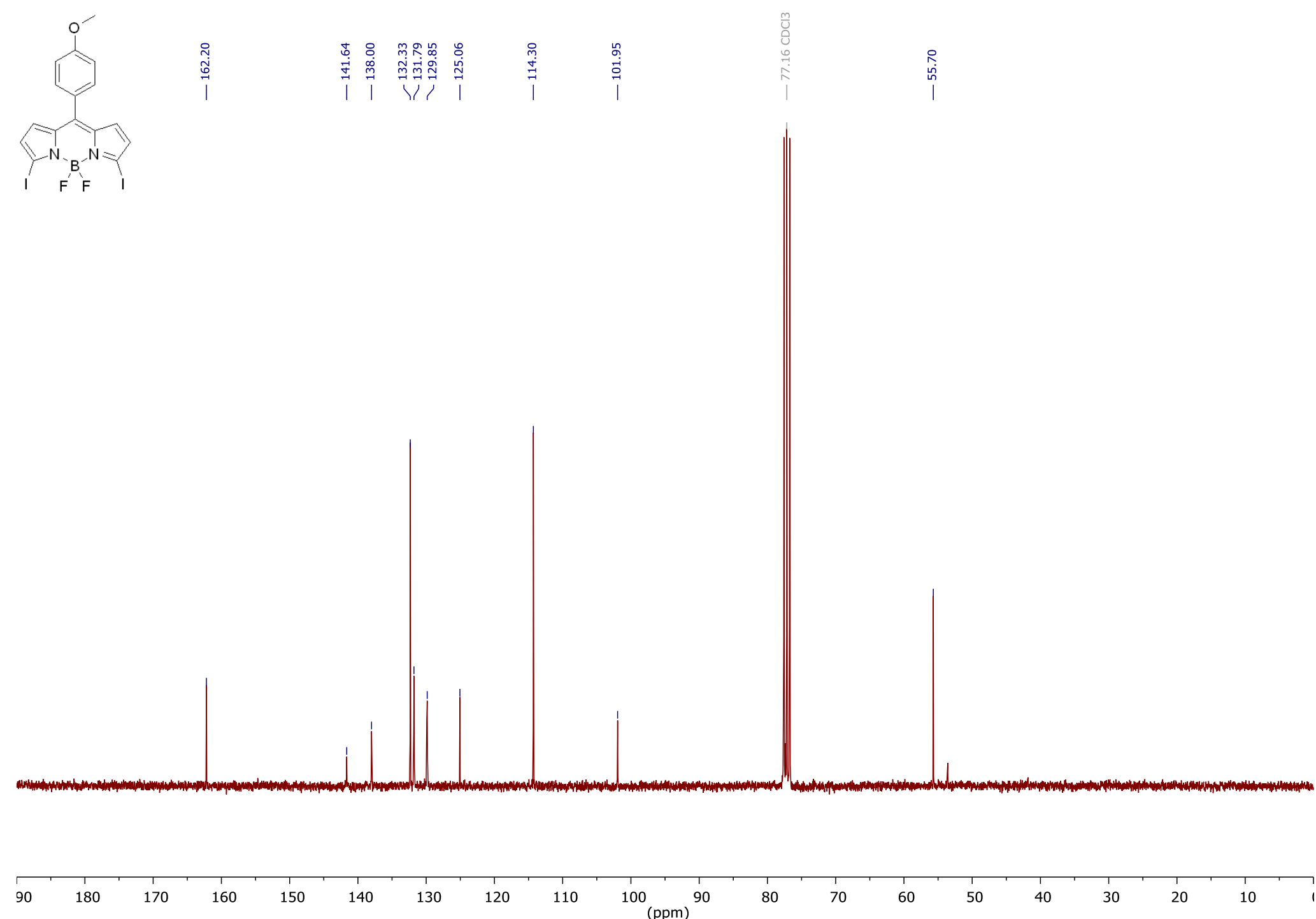
${ }^{19}$ F NMR (282 MHz, CDCl 3 ): 5,5-difluoro-3,7-diiodo-10-(4-methoxyphenyl)-5H-4 $\lambda^{4}, 5 \lambda^{4}$-dipyrrolo[1,2-c:2',1'-f][1,3,2]diazaborinine (3c)

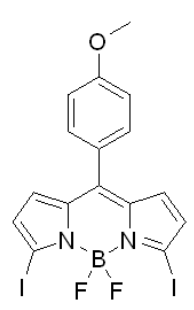

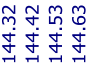

iil

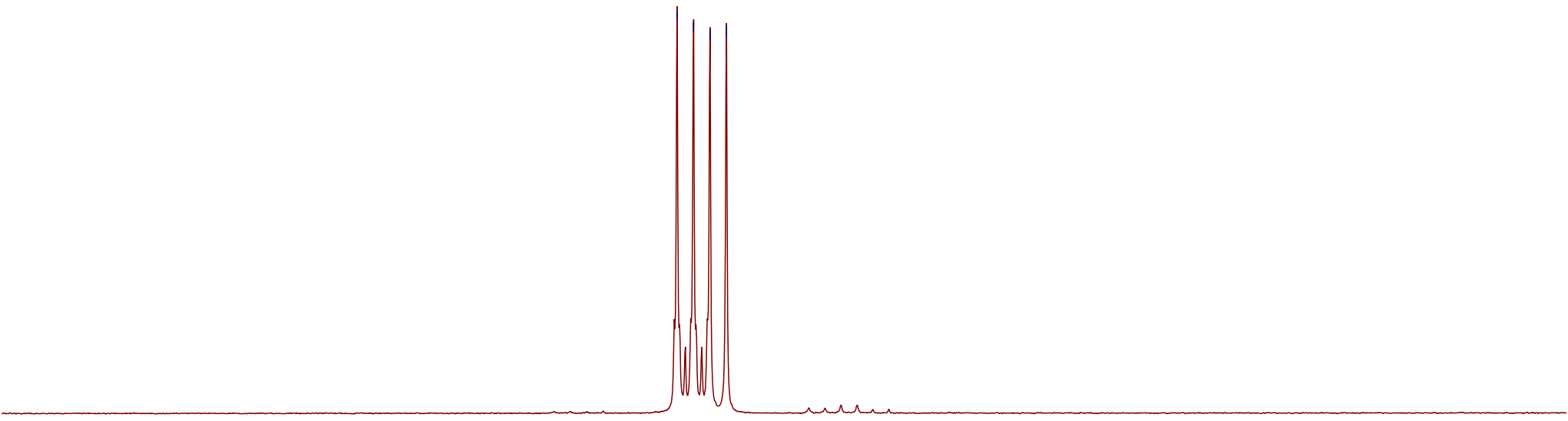

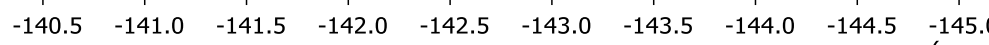
-145.0
(ppm) 
${ }^{11}$ B NMR (96 MHz, CDCl 3 ): 5,5-difluoro-3,7-diiodo-10-(4-methoxyphenyl)-5H-4 $\lambda^{4}, 5 \lambda^{4}$-dipyrrolo[1,2-c:2',1'-f][1,3,2] diazaborinine (3c)
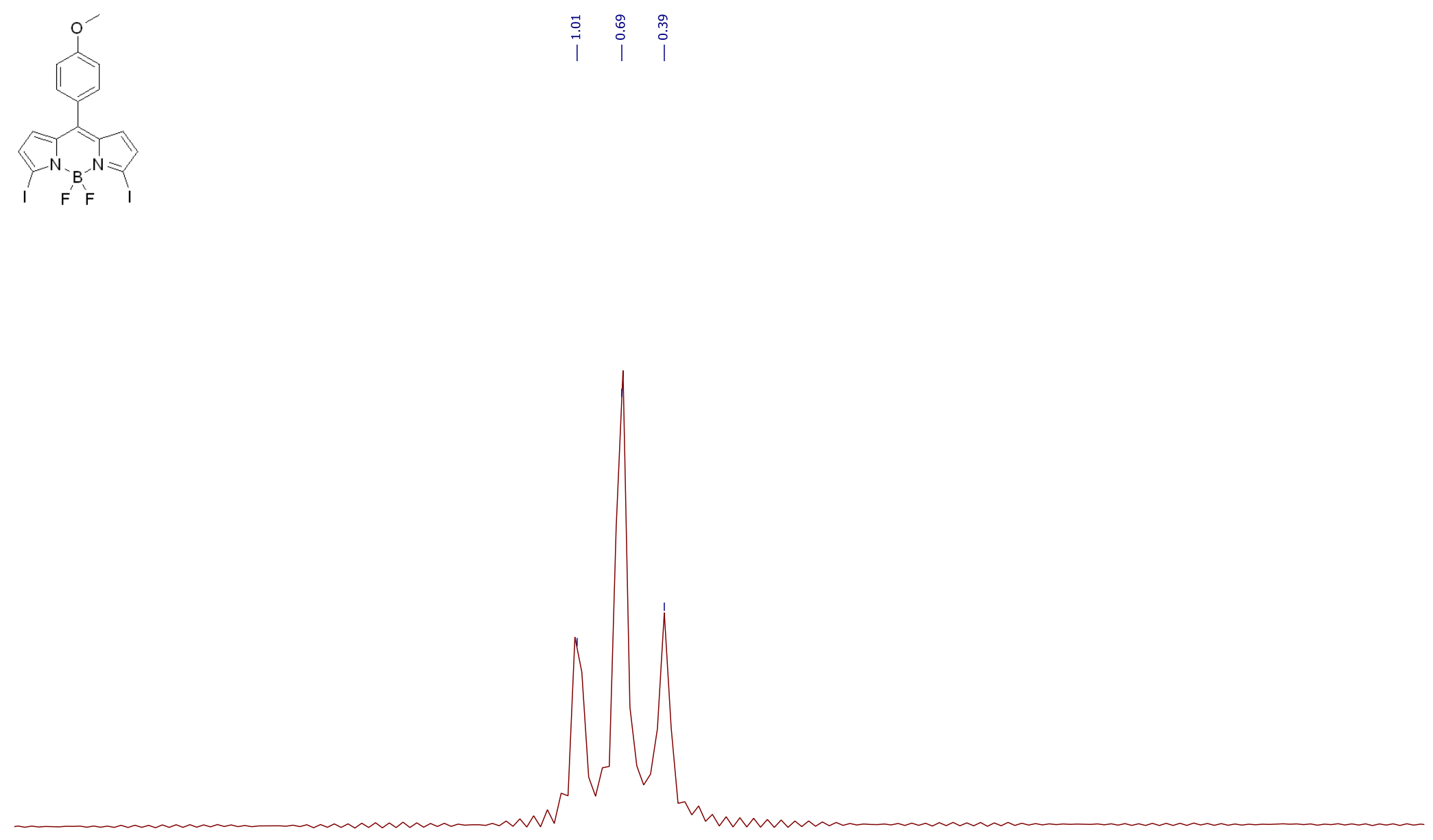

$\begin{array}{lllllllll}4.5 & 4.0 & 3.5 & 3.0 & 2.5 & 2.0 & 1.5 & 1.0 & 0.5\end{array}$

0.0
$(\mathrm{ppm})$

$-0.5 \quad-1.0$

$-1.5 \quad-2.0$

$-2.5$

$-3.0$

$-3.5 \quad-4.0$ 
${ }^{1}$ H NMR (300 MHz, CDCl 3 ): 5,5-difluoro-3,7-diiodo-10-(3-methoxyphenyl)-5H-4 $\lambda^{4}, 5 \lambda^{4}$-dipyrrolo[1,2-c:2',1'-f][1,3,2]diazaborinine (3d)

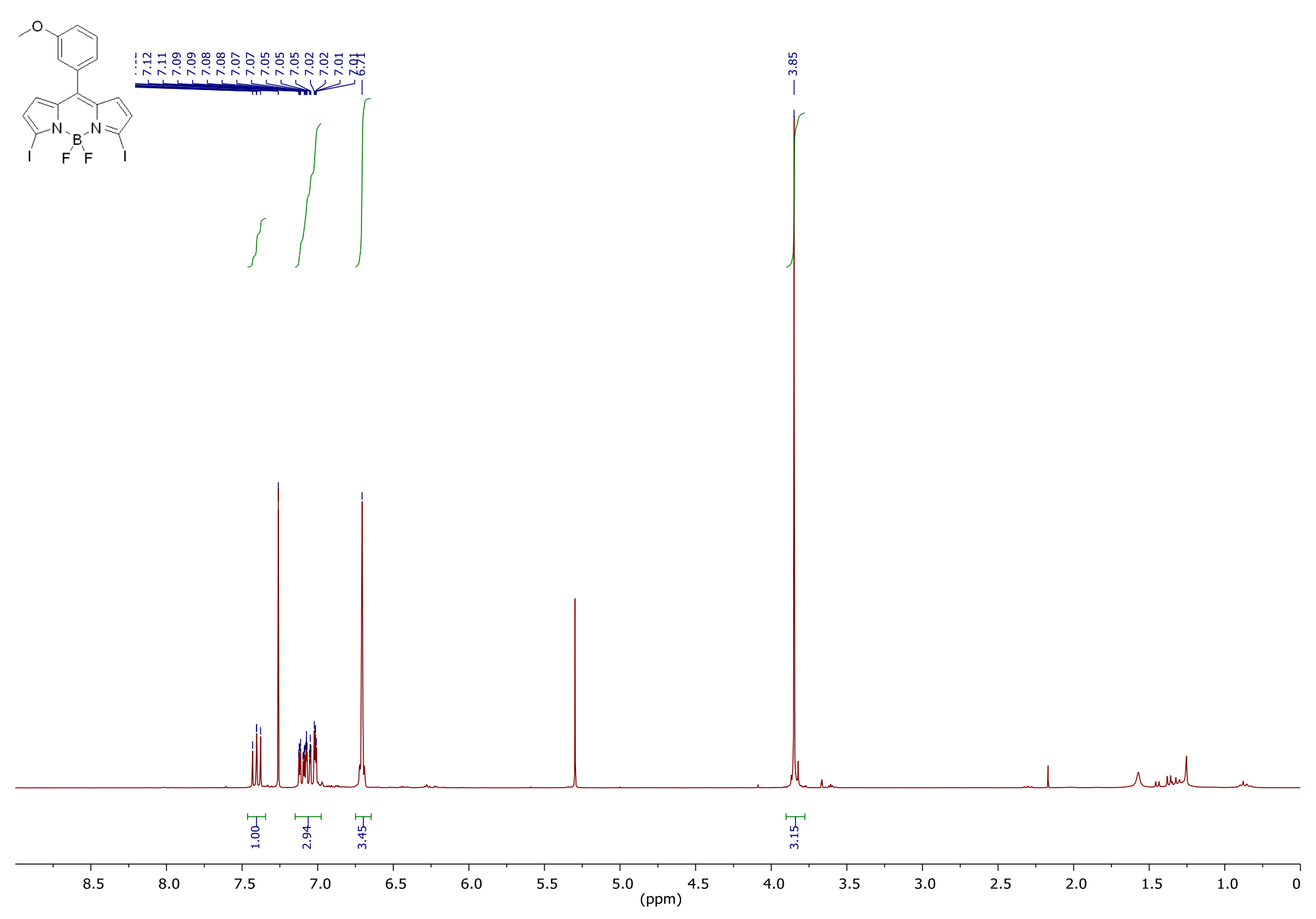


${ }^{13} \mathrm{C}$ NMR (75 MHz, CDCl 3 ): 5,5-difluoro-3,7-diiodo-10-(3-methoxyphenyl)-5H-4 $\lambda^{4}, 5 \lambda^{4}$-dipyrrolo[1,2-c:2',1'-f][1,3,2] diazaborinine (3d)

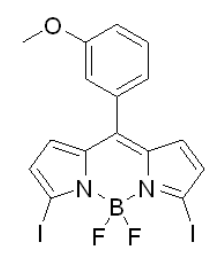

I F F I

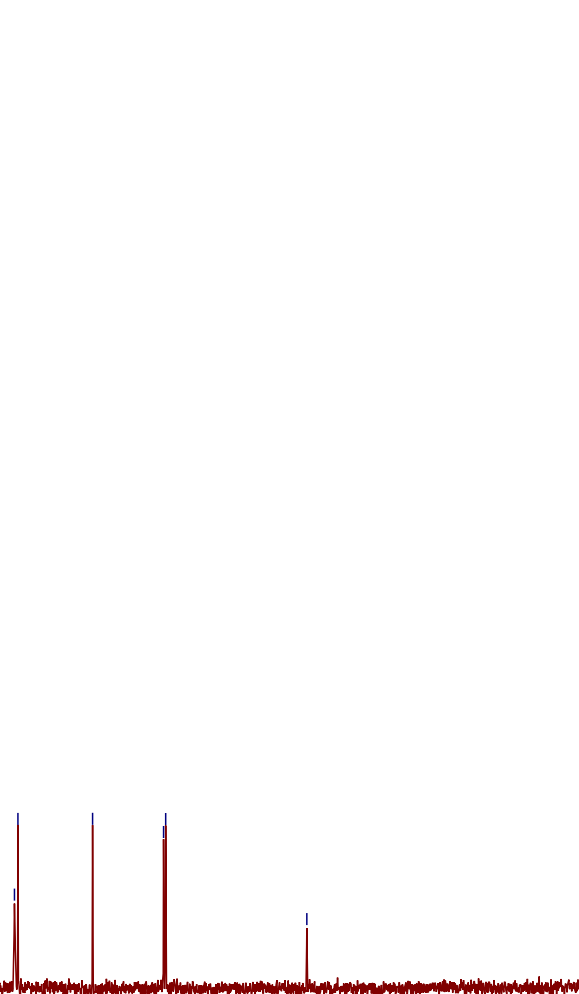

$90 \quad 180$ 
${ }^{19}$ F NMR (282 MHz, CDCl 3 ): 5,5-difluoro-3,7-diiodo-10-(3-methoxyphenyl)-5H-4 $\lambda^{4}, 5 \lambda^{4}$-dipyrrolo[1,2-c:2',1'-f][1,3,2] diazaborinine (3d)
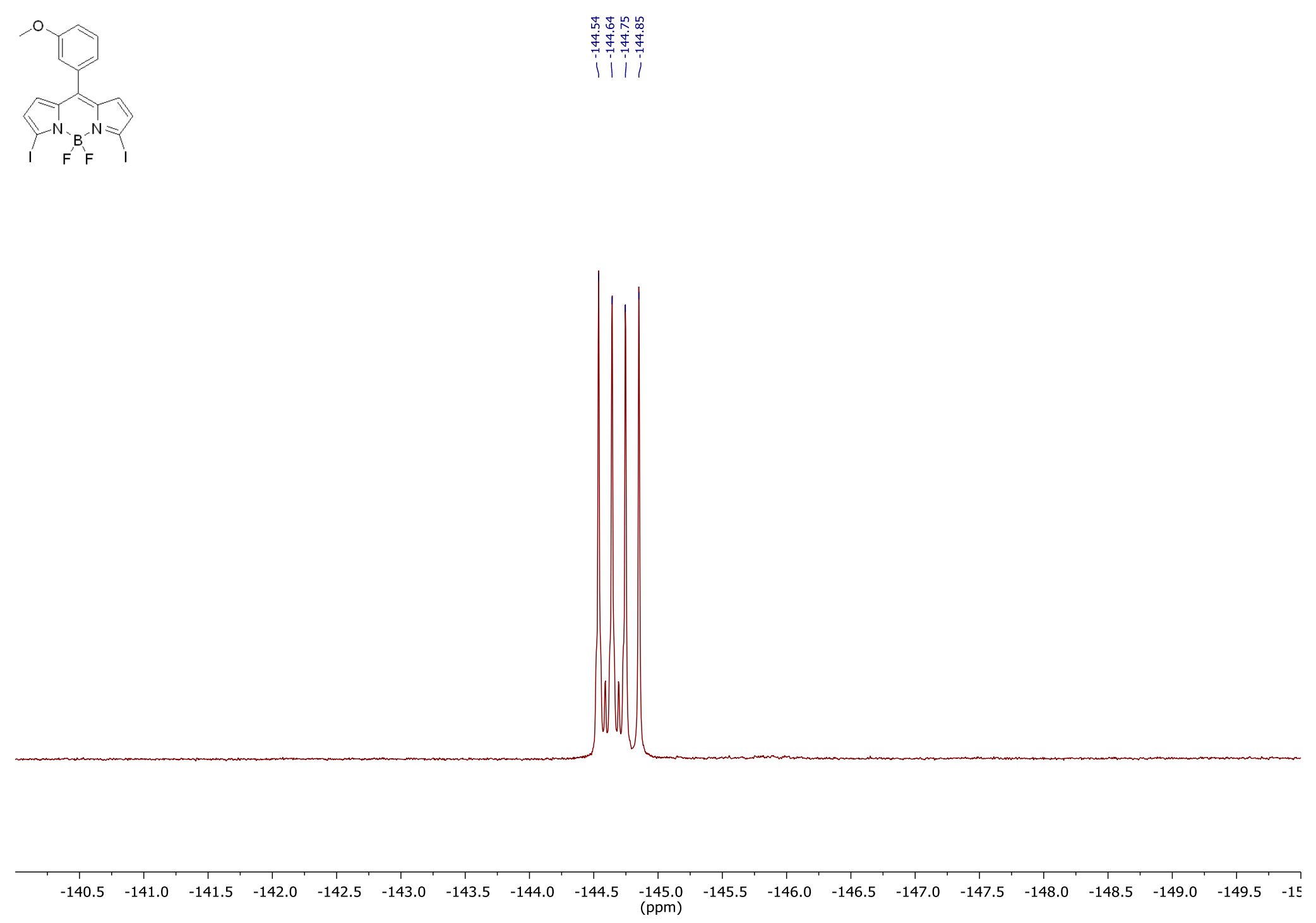
${ }^{11}$ B NMR (96 MHz, CDCl 3 ): 5,5-difluoro-3,7-diiodo-10-(3-methoxyphenyl)-5H-4 $\lambda^{4}, 5 \lambda^{4}$-dipyrrolo[1,2-c:2',1'-f][1,3,2] diazaborinine (3d)
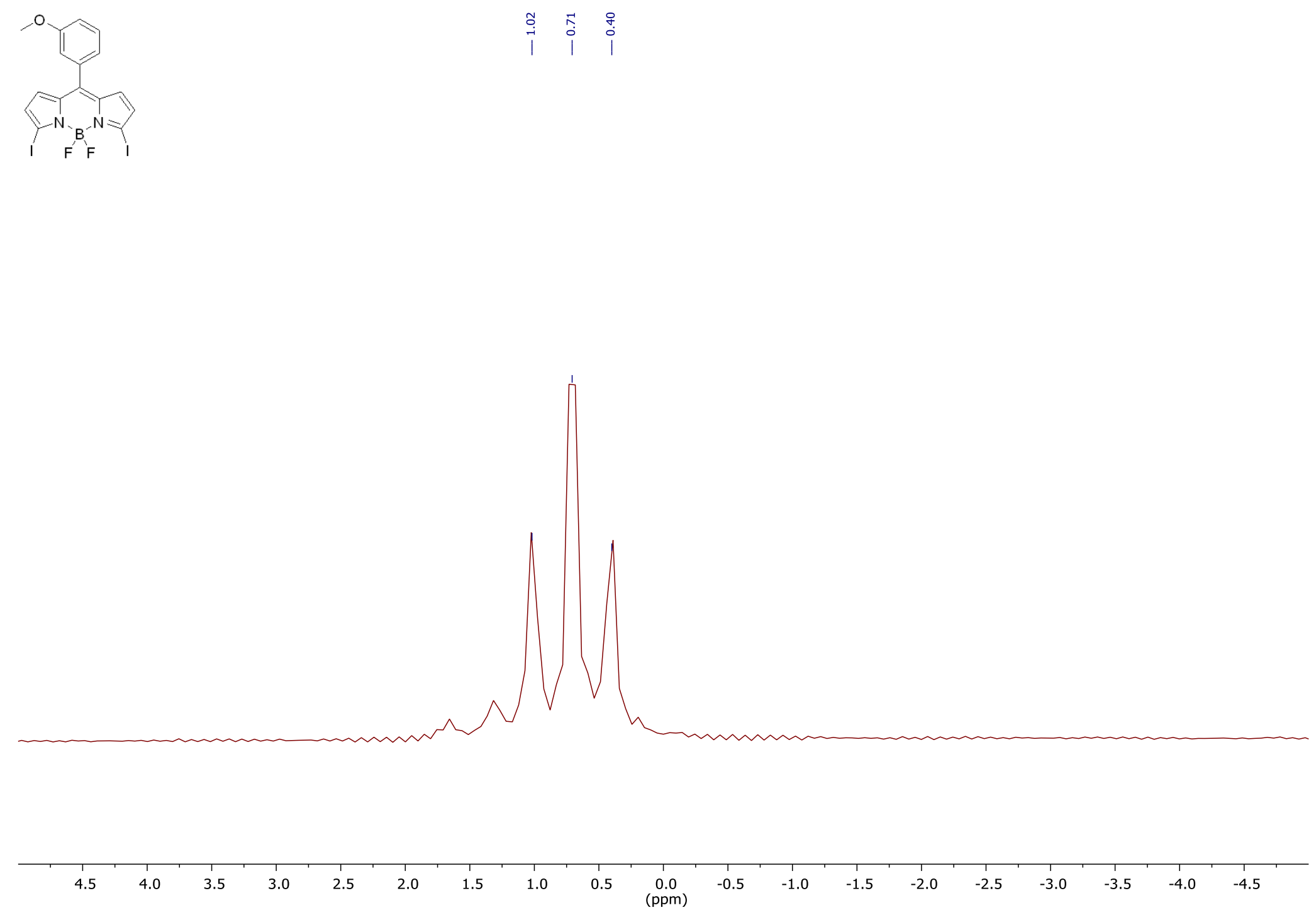
${ }^{1}$ H NMR (300 MHz, CDCl $)$ : Methyl 4-(5,5-difluoro-3,7-diphenyl-5H-4 $\lambda^{4}, 5 \lambda^{4}$-dipyrrolo[1,2-c:2',1'-f][1,3,2]diazaborinin-10-yl)benzoate (4)
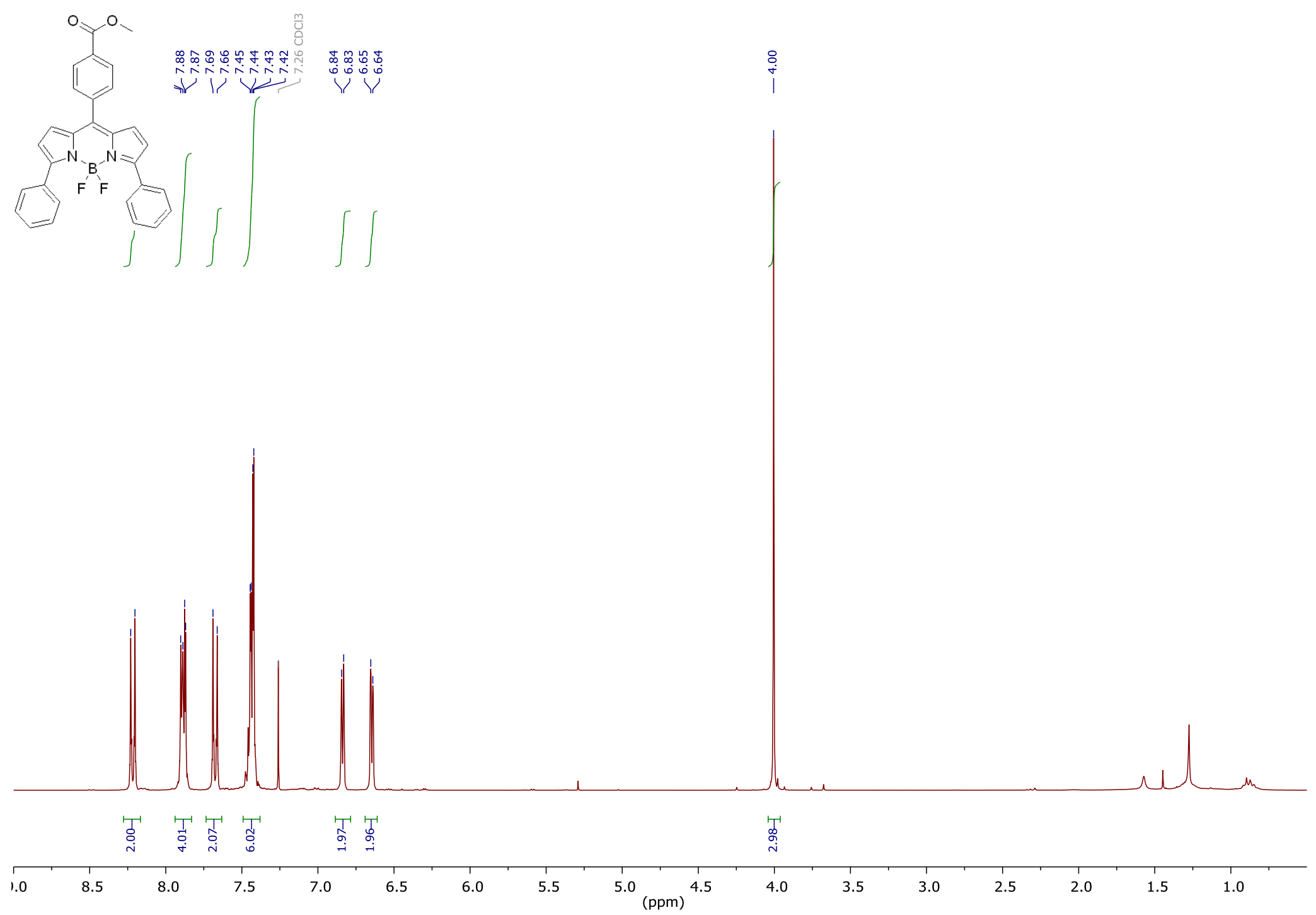
${ }^{13} \mathrm{C}$ NMR (75 MHz, CDCl 3 ): Methyl 4-(5,5-difluoro-3,7-diphenyl-5H-4 $\lambda^{4}, 5 \lambda^{4}$-dipyrrolo[1,2-c:2',1'-f][1,3,2] diazaborinin-10-yl)benzoate (4)
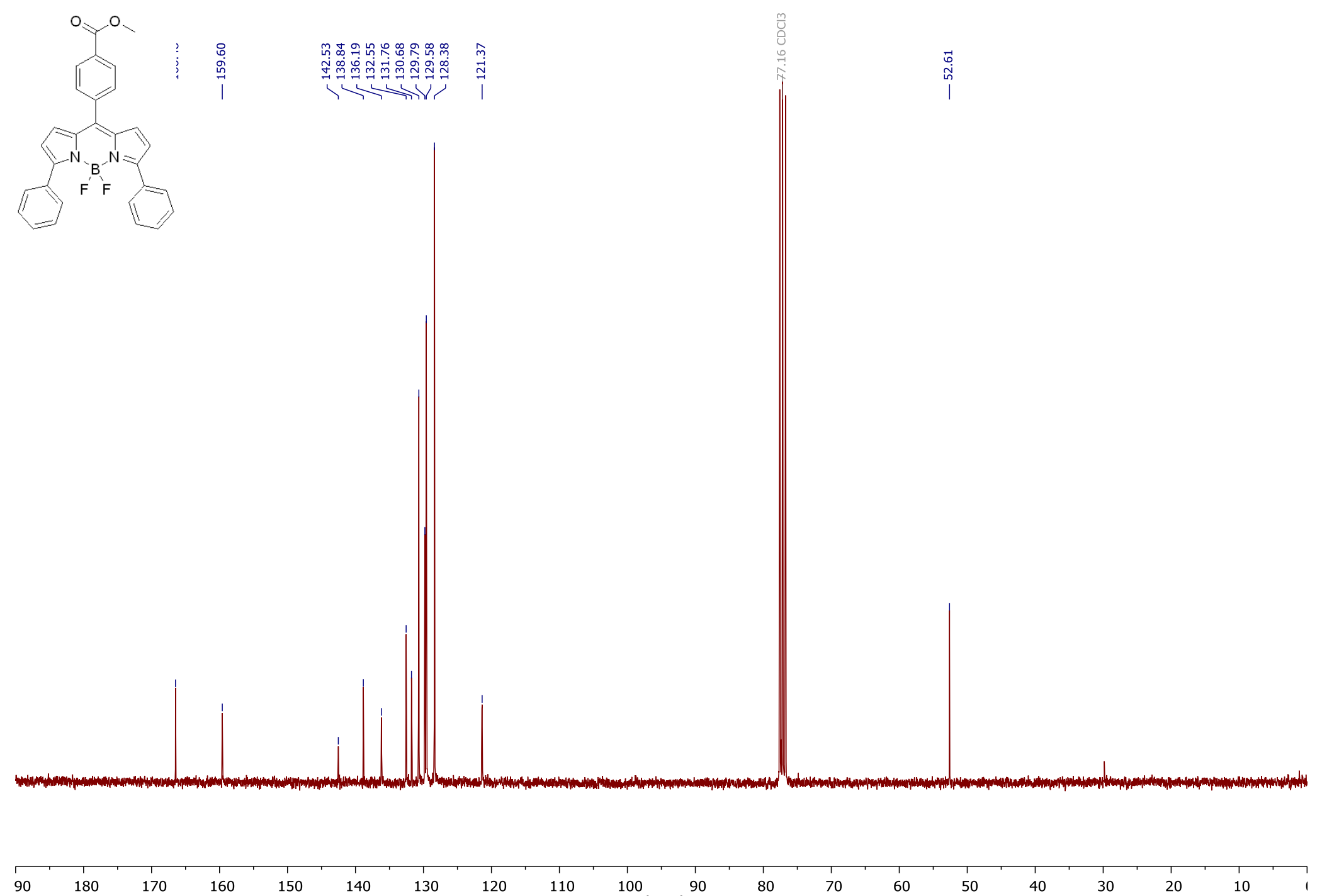

120
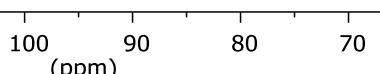

60
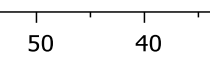
${ }^{19}$ F NMR (282 MHz, CDCl 3$)$ : Methyl 4-(5,5-difluoro-3,7-diphenyl-5H-4 $\lambda^{4}, 5 \lambda^{4}$-dipyrrolo[1,2-c:2',1'-f][1,3,2]diazaborinin-10-yl)benzoate (4)

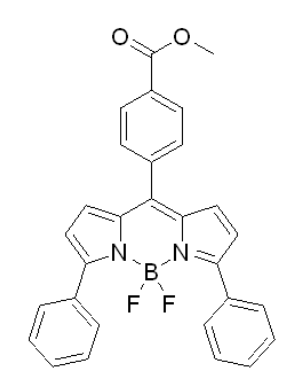


${ }^{11}$ B NMR (96 MHz, CDCl 3 ): Methyl 4-(5,5-difluoro-3,7-diphenyl-5H-4 $\lambda^{4}, 5 \lambda^{4}$-dipyrrolo[1,2-c:2',1'-f][1,3,2]diazaborinin-10-yl)benzoate (4)

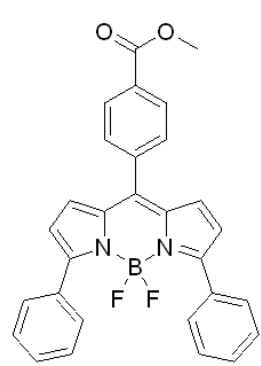

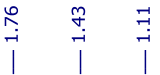

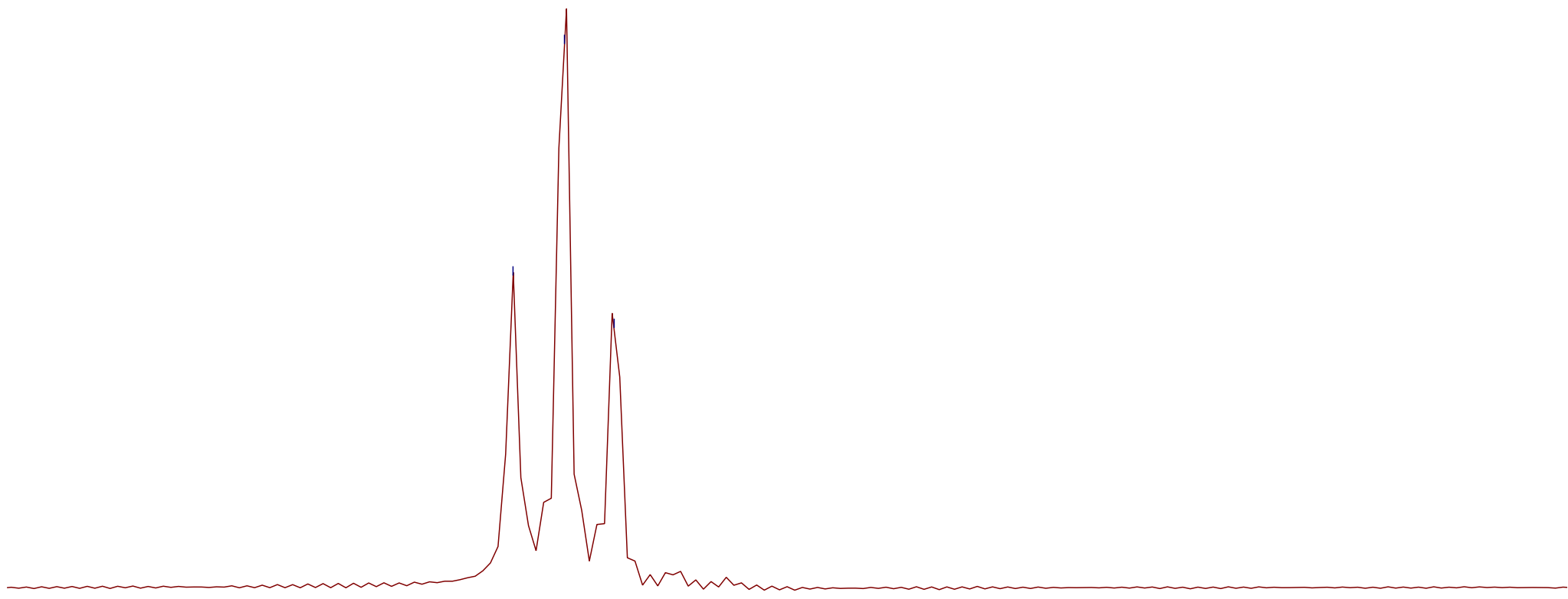

$4.5 \quad 4.0$

3.5

$2.5 \quad 2.0$

1.5

$\begin{array}{lc}0.5 & 0.0 \\ (\mathrm{ppm})\end{array}$

$\begin{array}{ll}-0.5 & -1.0\end{array}$

$-1.5 \quad-2.0$

$-2.5$

$-3.0$

$\begin{array}{ll}-3.5 & -4.0\end{array}$

$-4.5$ 
${ }^{1} \mathrm{H}$ NMR (300 MHz, CDCl 3 ): methyl 4-(3-butyl-5,5-difluoro-7-phenyl-5H-5 $\lambda^{4}, 6 \lambda^{4}$-dipyrrolo[1,2-c:2',1'-f][1,3,2] diazaborinin-10yl)benzoate (5)

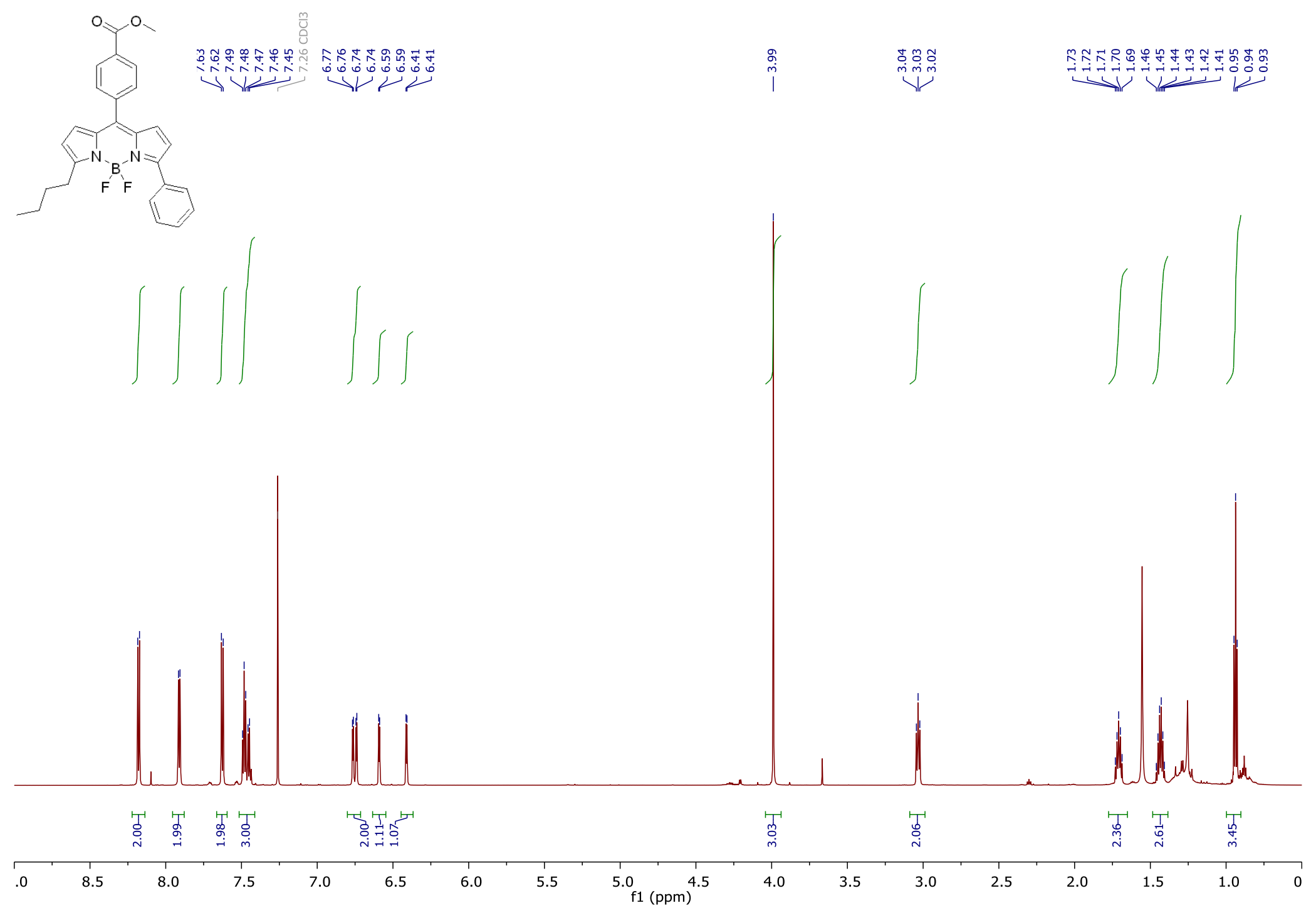


${ }^{13} \mathrm{C}$ NMR (176 MHz, CDCl 3$)$ : methyl 4-(3-butyl-5,5-difluoro-7-phenyl-5H-5 $\lambda^{4}, 6 \lambda^{4}$-dipyrrolo[1,2-c:2',1'-f][1,3,2]diazaborinin-10yl)benzoate (5)

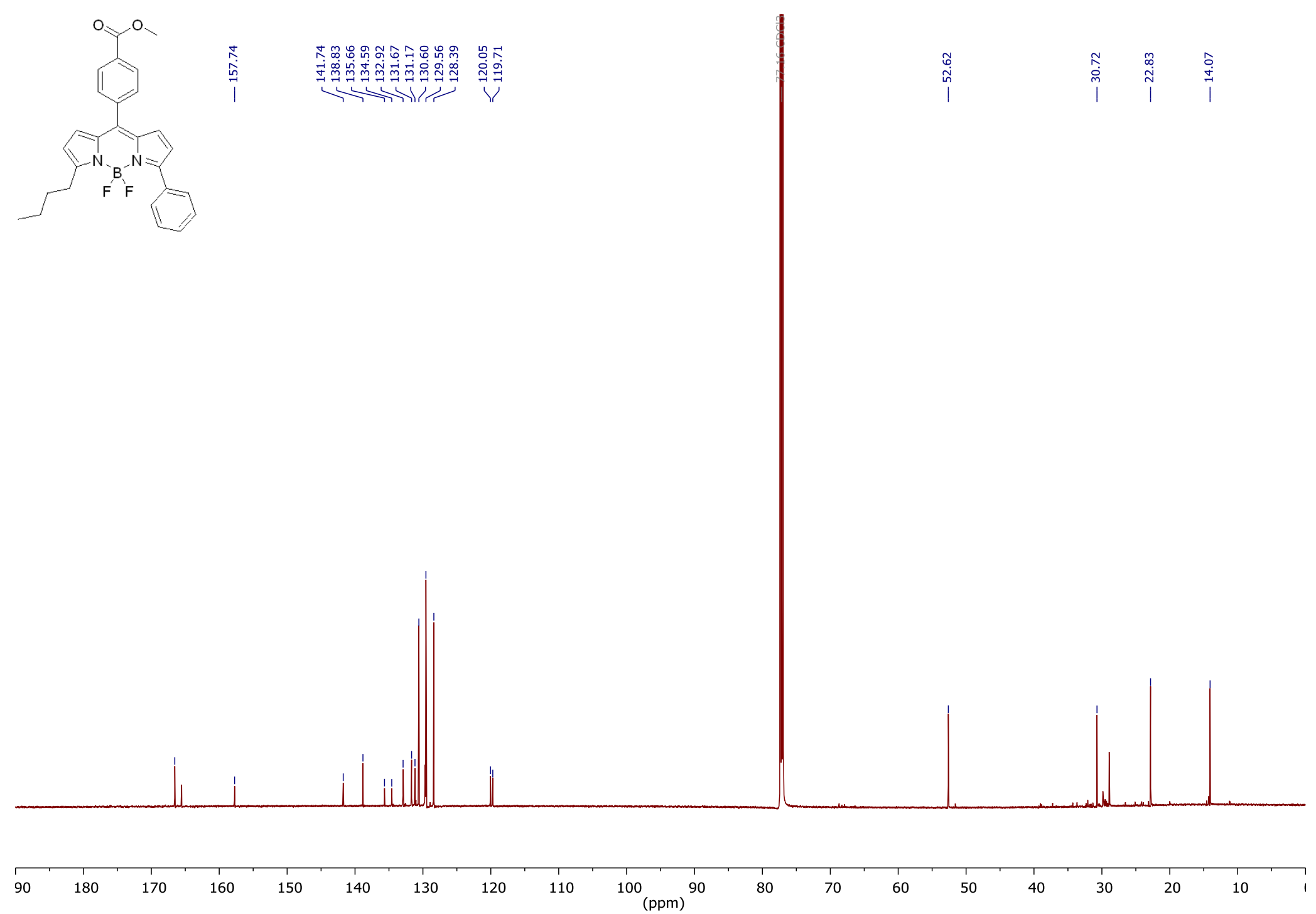


${ }^{19}$ F NMR (282 MHz, CDCl $)$ : methyl 4-(3-butyl-5,5-difluoro-7-phenyl-5H-5 $\lambda^{4}, 6 \lambda^{4}$-dipyrrolo[1,2-c $: 2$ ',1'-f][1,3,2]diazaborinin-10yl)benzoate (5)

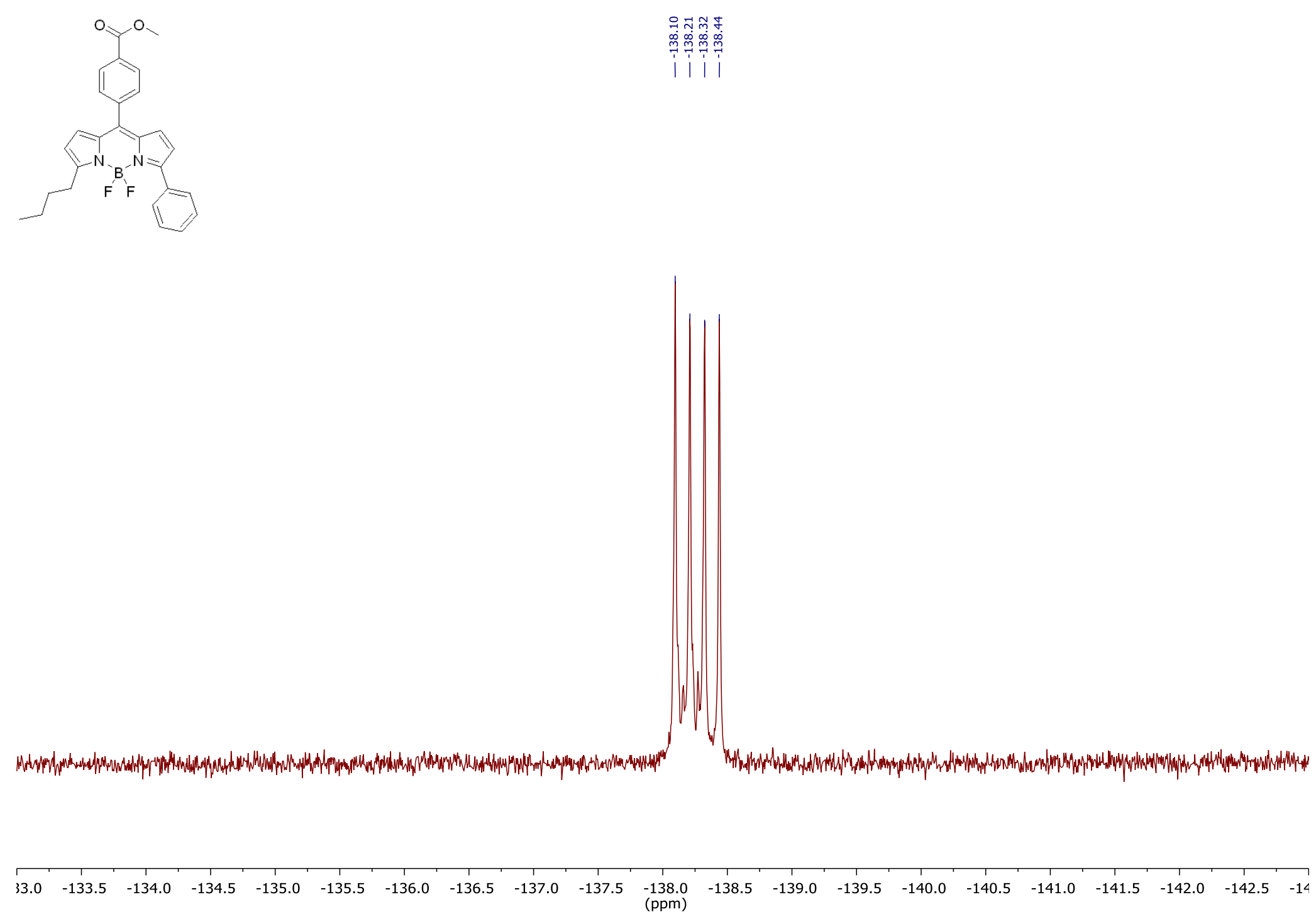


${ }^{11}$ B NMR (96 MHz, CDCl $)$ : methyl 4-(3-butyl-5,5-difluoro-7-phenyl-5H-5 $\lambda^{4}, 6 \lambda^{4}$-dipyrrolo[1,2-c:2',1'-f][1,3,2]diazaborinin-10-yl)benzoate (5)
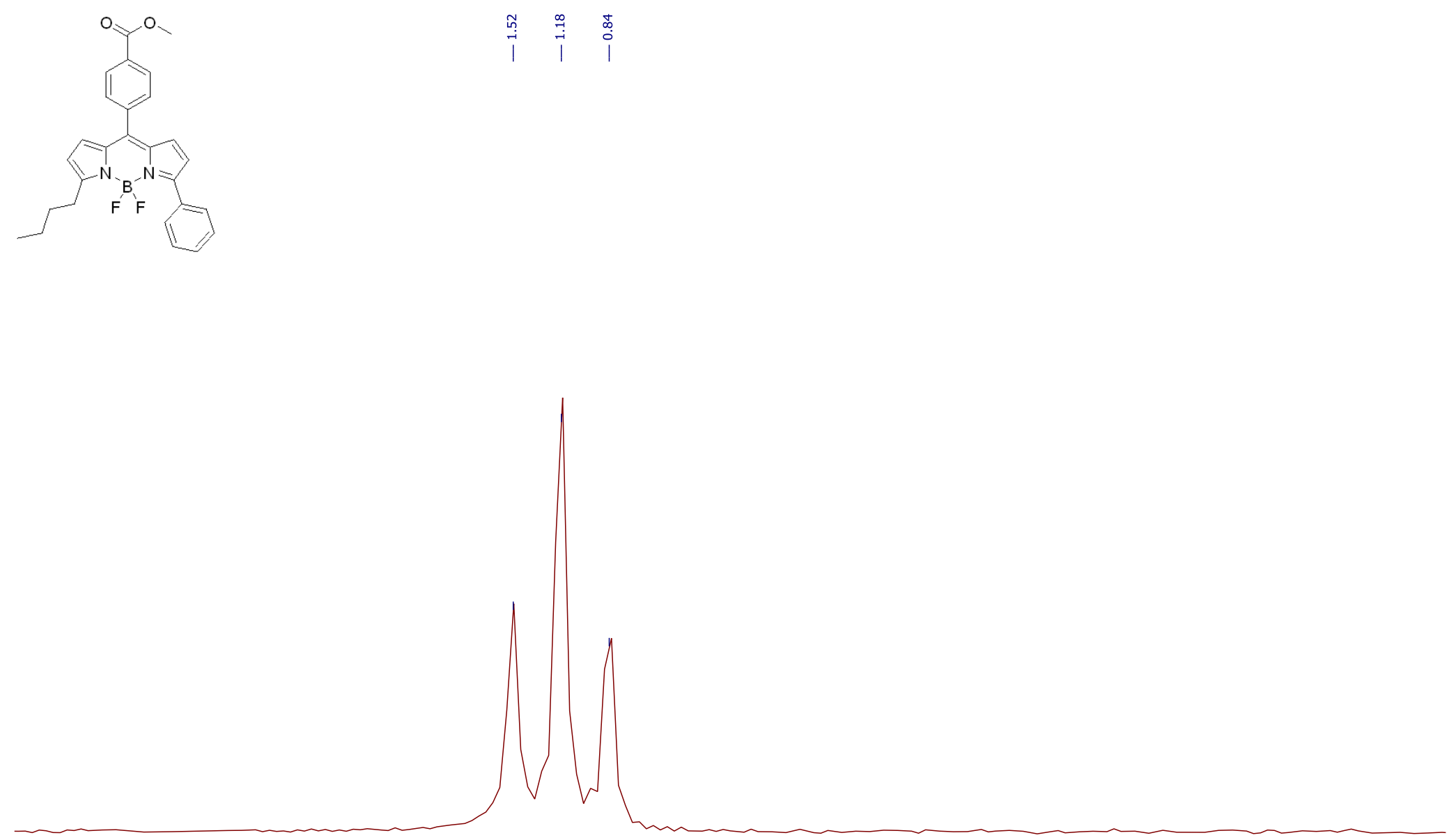
${ }^{1}$ H NMR (300 MHz, CDCl $)$ : Methyl 4-(5,5-difluoro-3,7-di((E)-styryl)-5H-4 $\lambda^{4}, 5 \lambda^{4}$-dipyrrolo[1,2-c:2',1'-f][1,3,2]diazaborinin-10yl)benzoate (6)

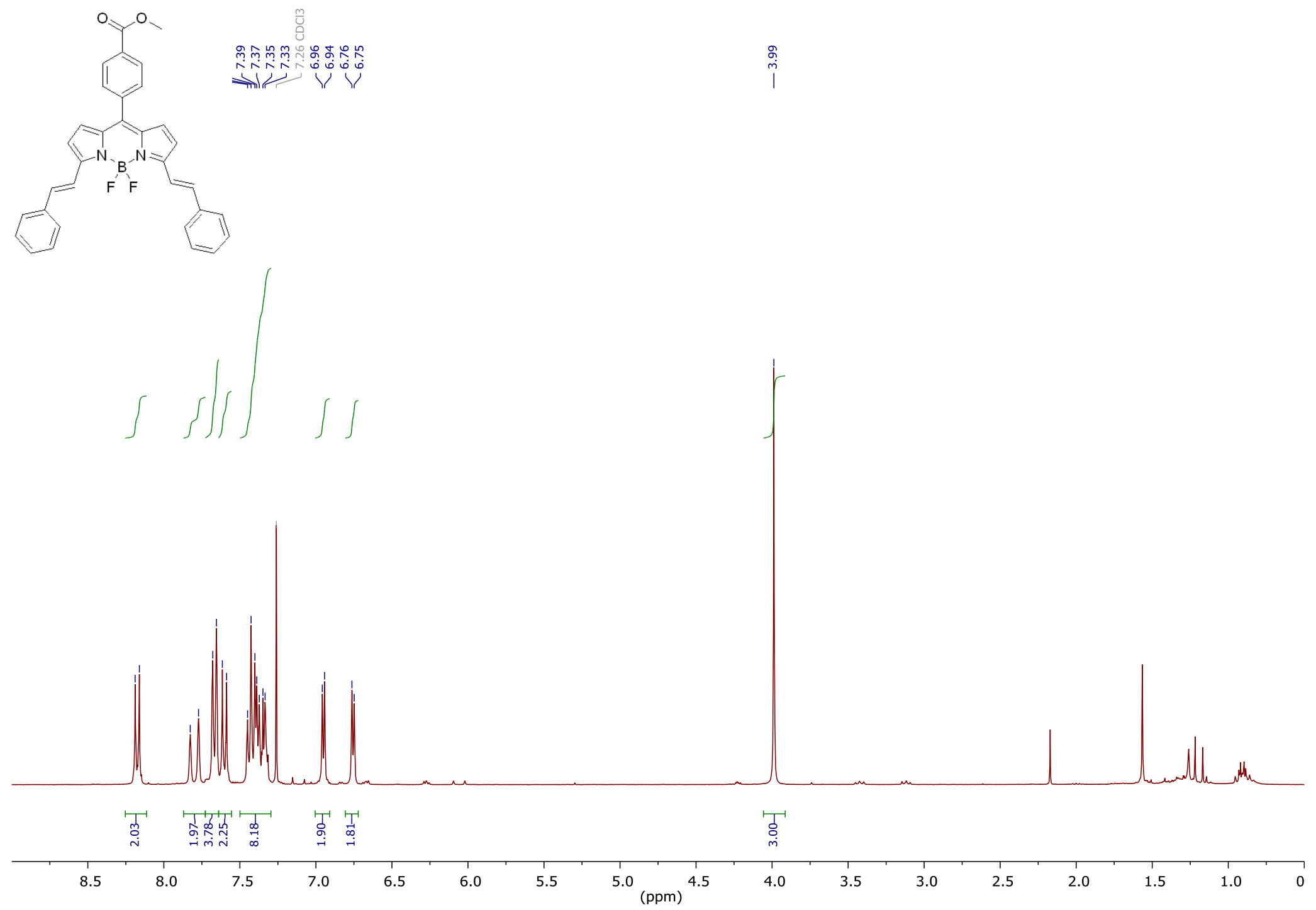


${ }^{13} \mathrm{C}$ NMR (75 MHz, CDCl 3$)$ : Methyl 4-(5,5-difluoro-3,7-di((E)-styryl)-5H-4 $\lambda^{4}, 5 \lambda^{4}$-dipyrrolo[1,2-c:2',1'-f][1,3,2]diazaborinin-10yl)benzoate (6)
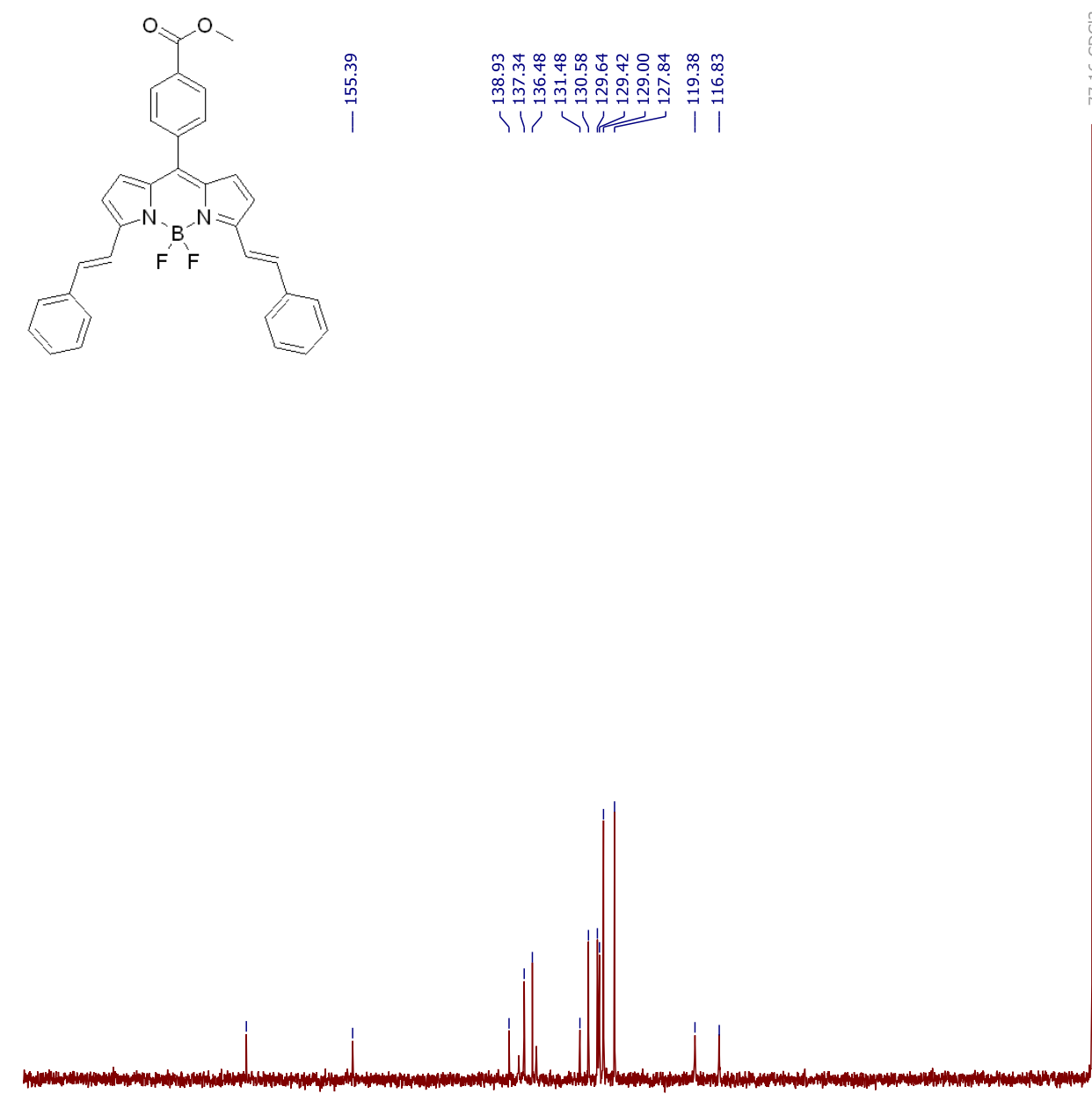
${ }^{19}$ F NMR (282 MHz, CDCl 3$)$ : Methyl 4-(5,5-difluoro-3,7-di((E)-styryl)-5H-4 $\lambda^{4}, 5 \lambda^{4}$-dipyrrolo[1,2-c:2',1'-f $][1,3,2]$ diazaborinin-10yl)benzoate (6)

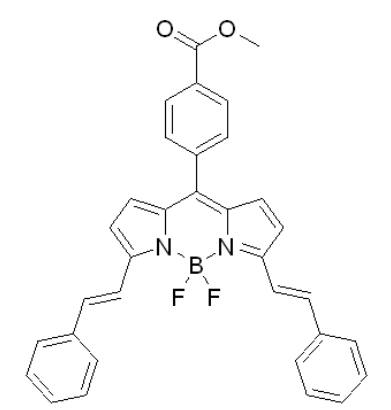

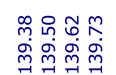

$$
\begin{aligned}
& \text { i i }
\end{aligned}
$$

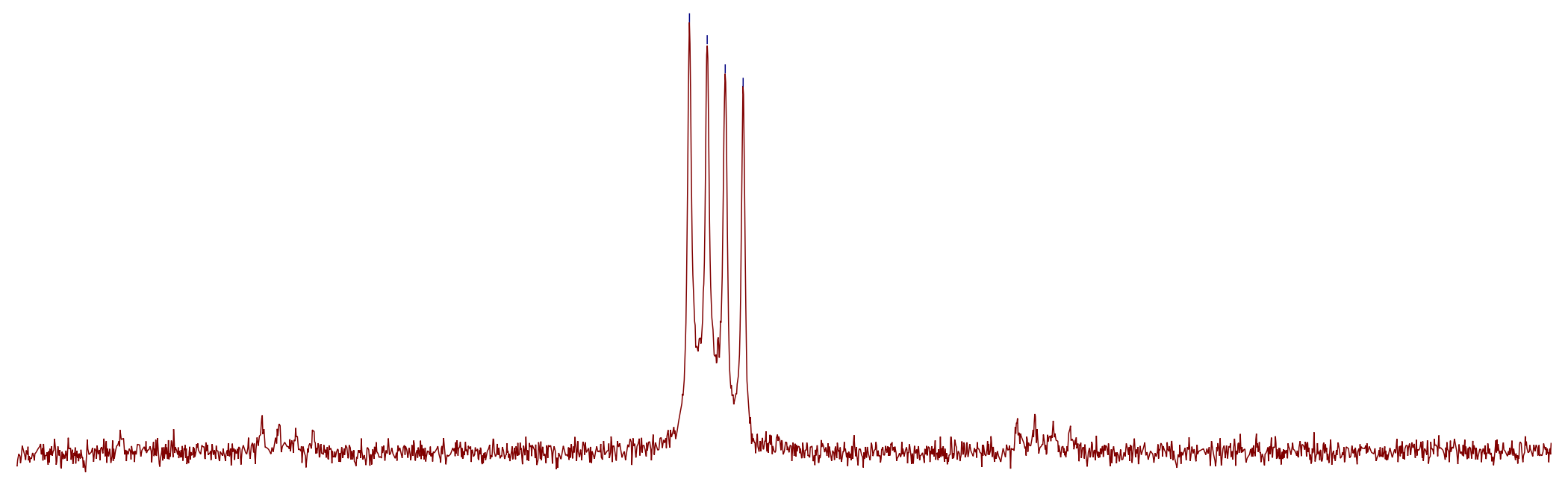

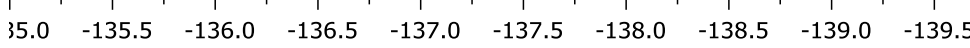


${ }^{11}$ B NMR (96 MHz, CDCl 3$)$ : Methyl 4-(5,5-difluoro-3,7-di((E)-styryl)-5H-4 $\lambda^{4}, 5 \lambda^{4}$-dipyrrolo[1,2-c:2',1'-f][1,3,2]diazaborinin-10yl)benzoate (6)

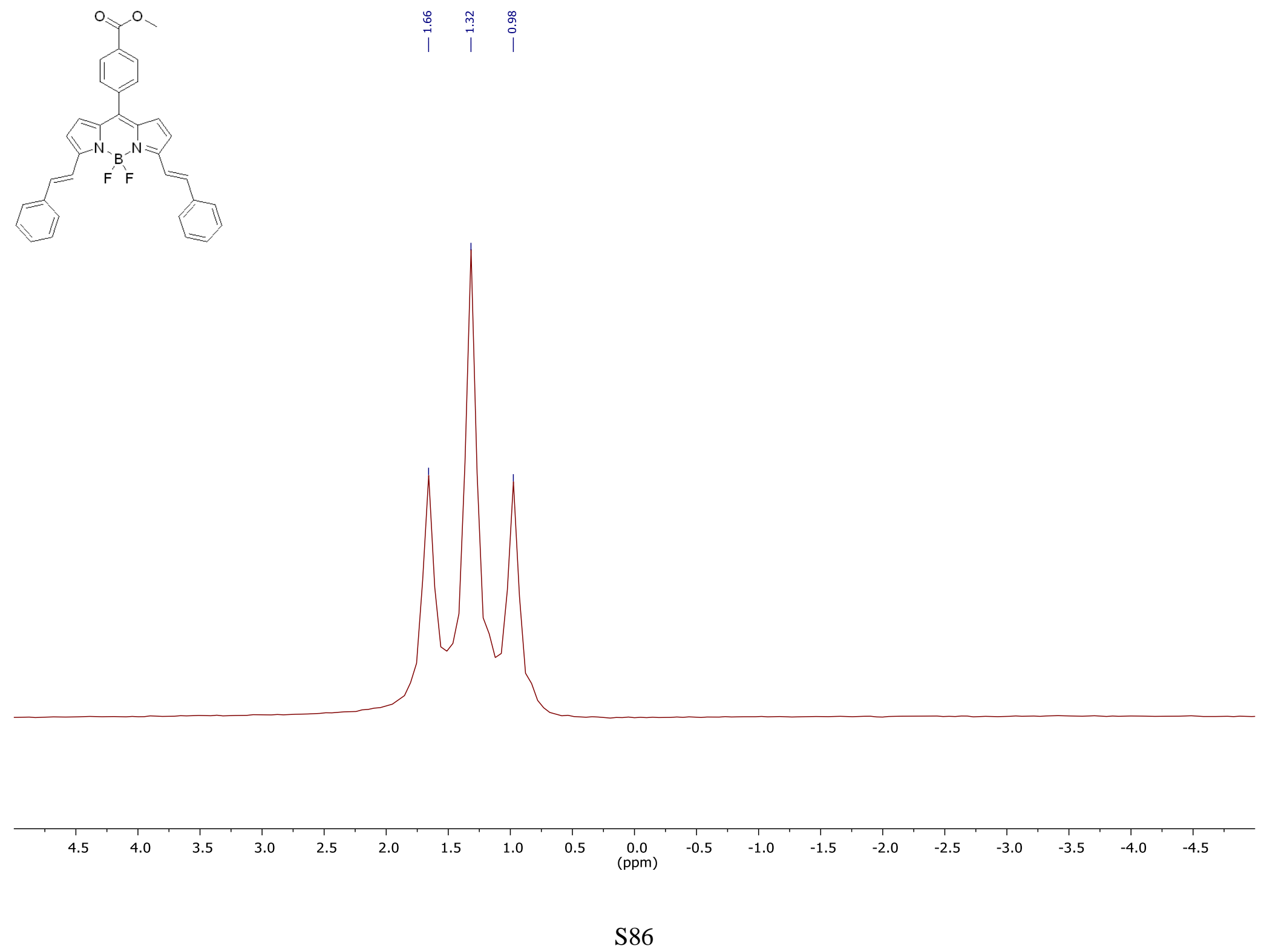


${ }^{1}$ H NMR (300 MHz, CDCl 3 ): Methyl 4-(5,5-difluoro-3,7-bis(phenylethynyl)-5H-4 $\lambda^{4}, 5 \lambda^{4}$-dipyrrolo[1,2-c:2',1'-f][1,3,2]diazaborinin-10yl)benzoate (7)

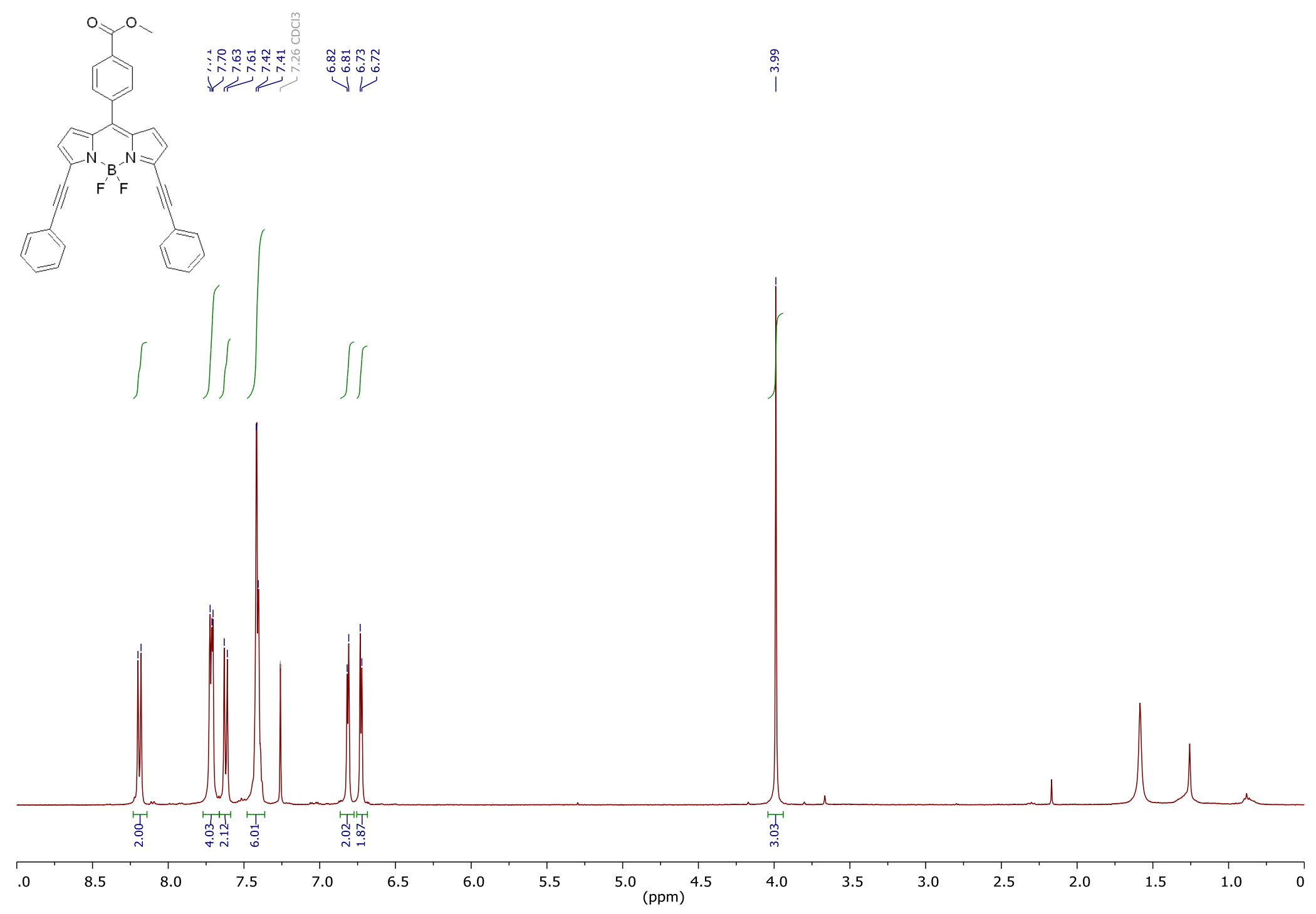


${ }^{13} \mathrm{C}$ NMR (75 MHz, CDCl 3$)$ : Methyl 4-(5,5-difluoro-3,7-bis(phenylethynyl)-5H-4 $\lambda^{4}, 5 \lambda^{4}$-dipyrrolo[1,2-c:2',1'-f][1,3,2]diazaborinin-10yl)benzoate (7)

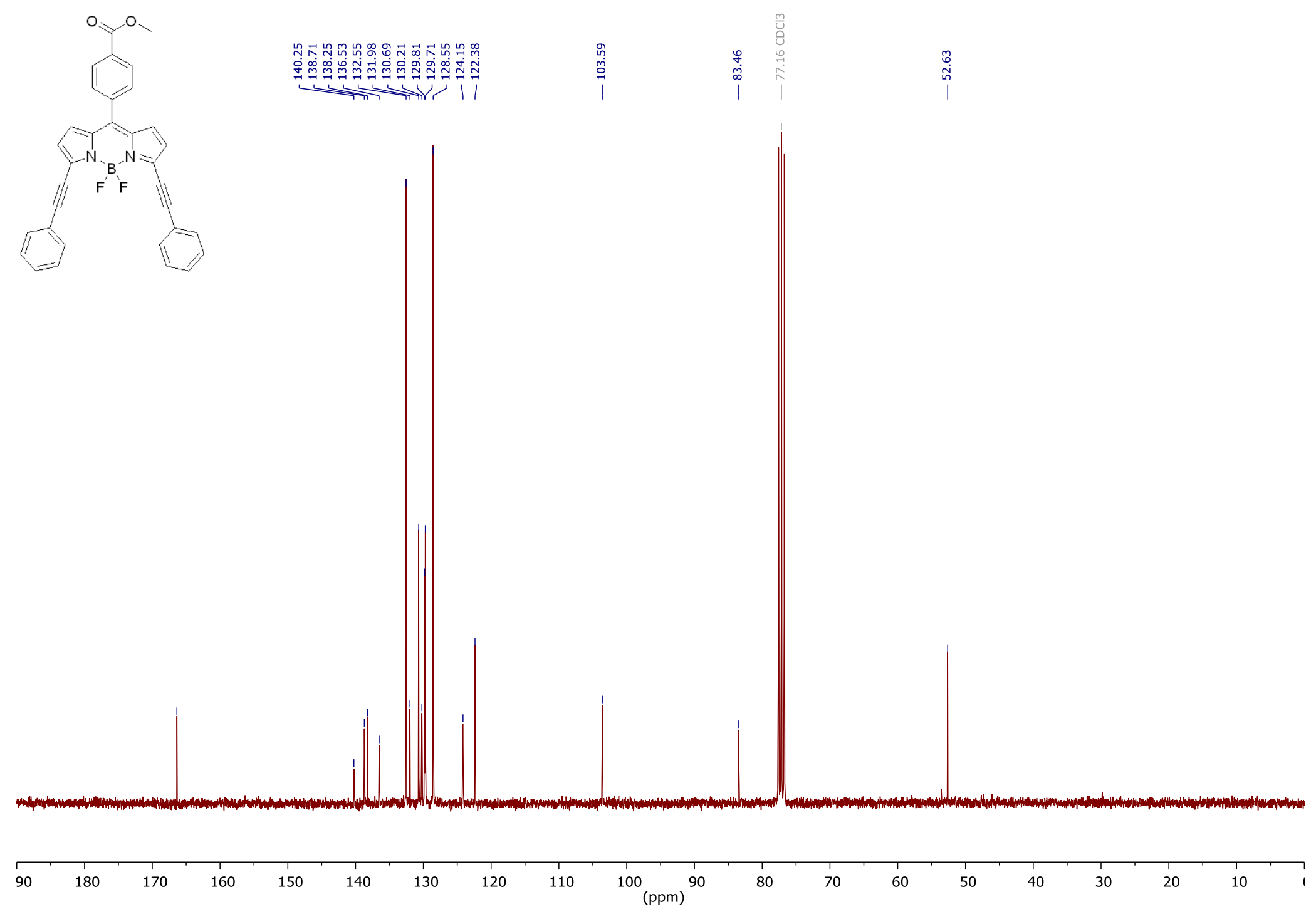


${ }^{19}$ F NMR (282 MHz, CDCl $)$ : Methyl 4-(5,5-difluoro-3,7-bis(phenylethynyl)-5H-4 $\lambda^{4}, 5 \lambda^{4}$-dipyrrolo[1,2-c:2',1'-f $][1,3,2]$ diazaborinin-10yl)benzoate (7)

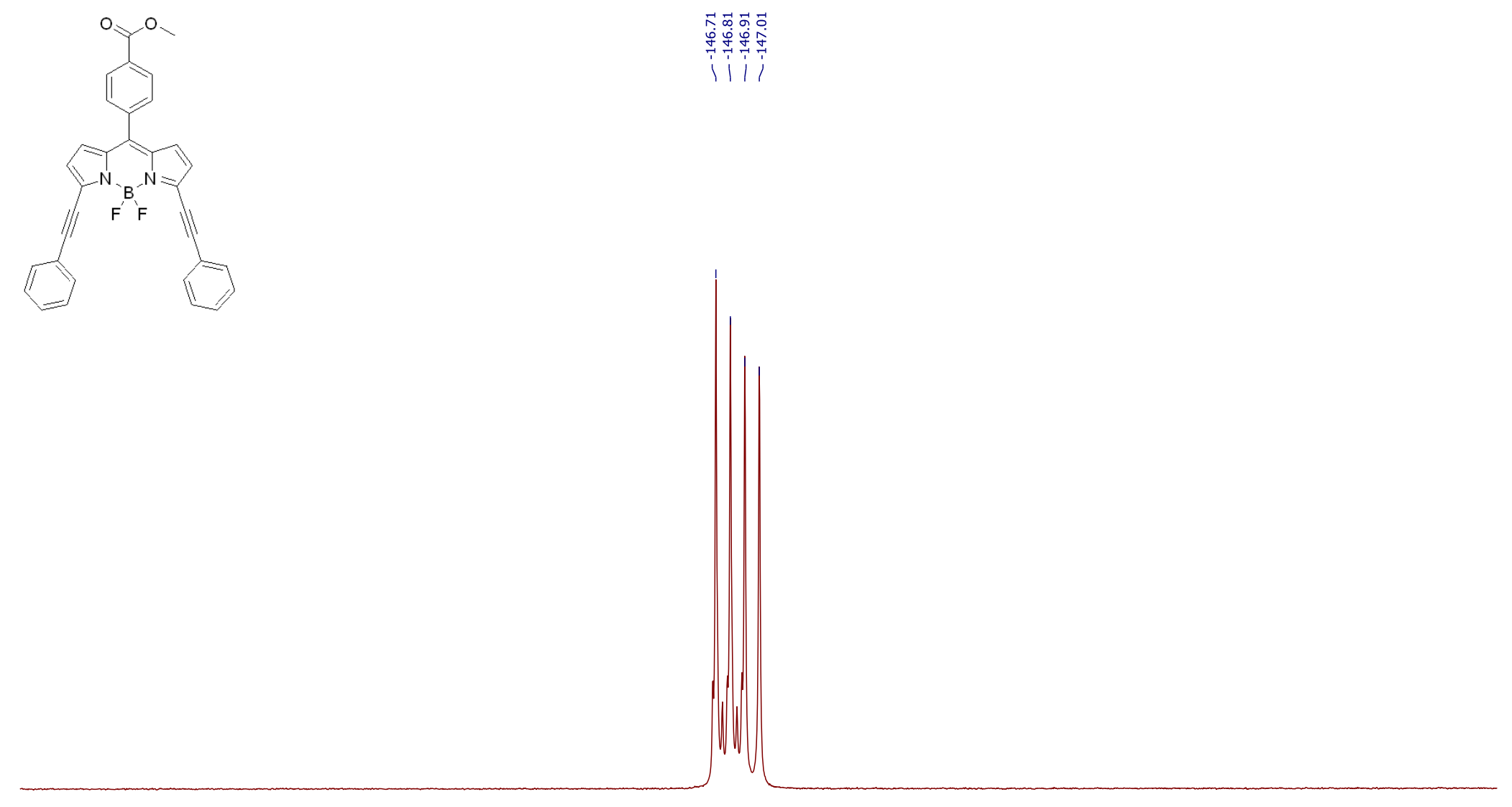

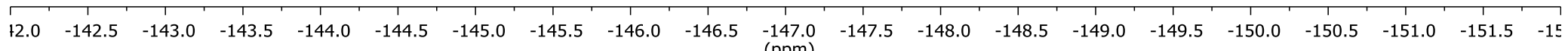


${ }^{11}$ B NMR (96 MHz, CDCl 3 ): Methyl 4-(5,5-difluoro-3,7-bis(phenylethynyl)-5H-4 $\lambda^{4}, 5 \lambda^{4}$-dipyrrolo[1,2-c:2',1'-f $][1,3,2]$ diazaborinin-10yl)benzoate (7)

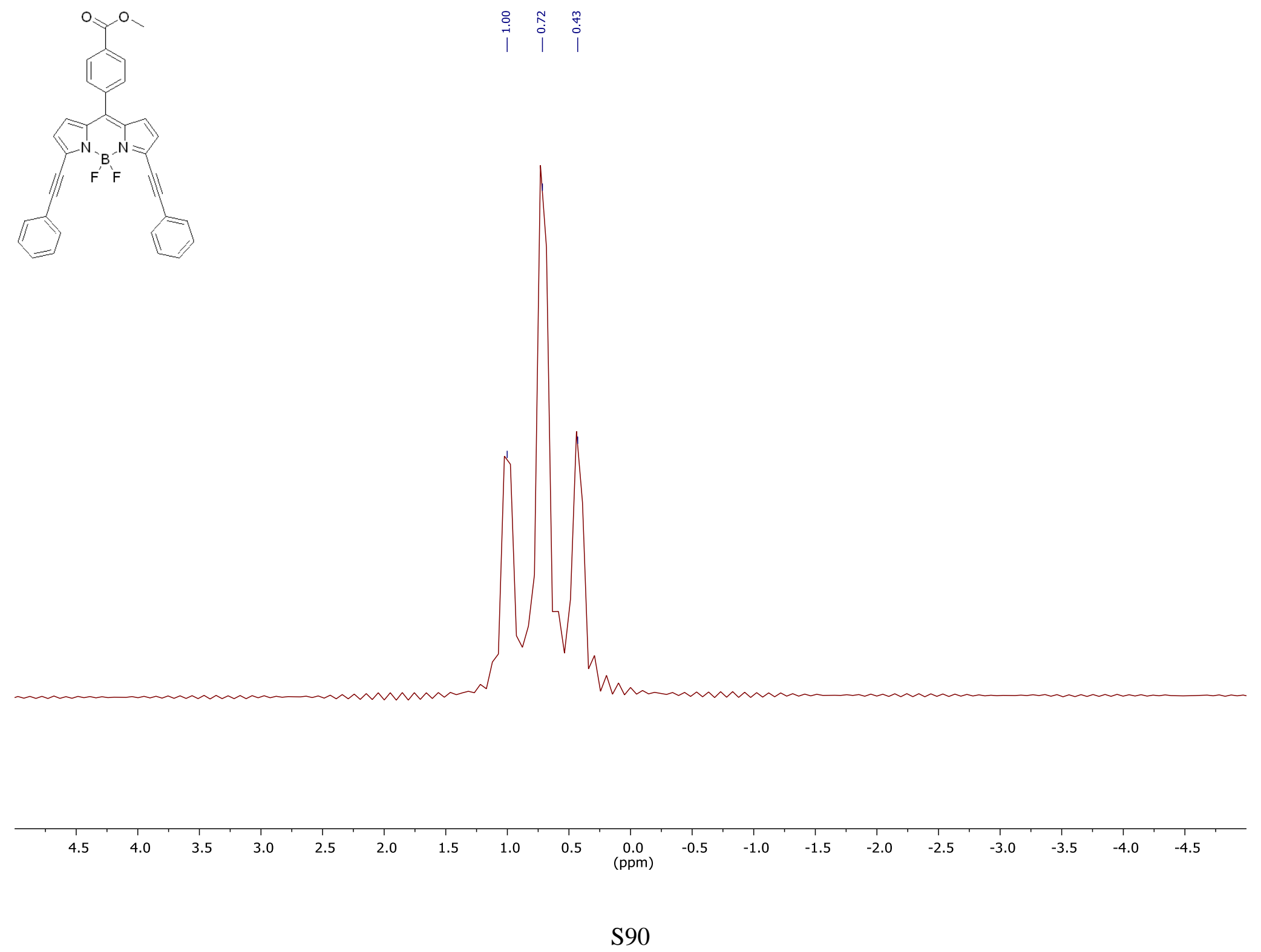


${ }^{1}$ H NMR (400 MHz, CDCl $)$ ) 4-(3-chloro-5,5-difluoro-7-(phenylethynyl)-5H-4 $\lambda^{4}, 5 \lambda^{4}$-dipyrrolo[1,2-c:2',1'-f][1,3,2]diazaborinin-10yl)benzoate (8)

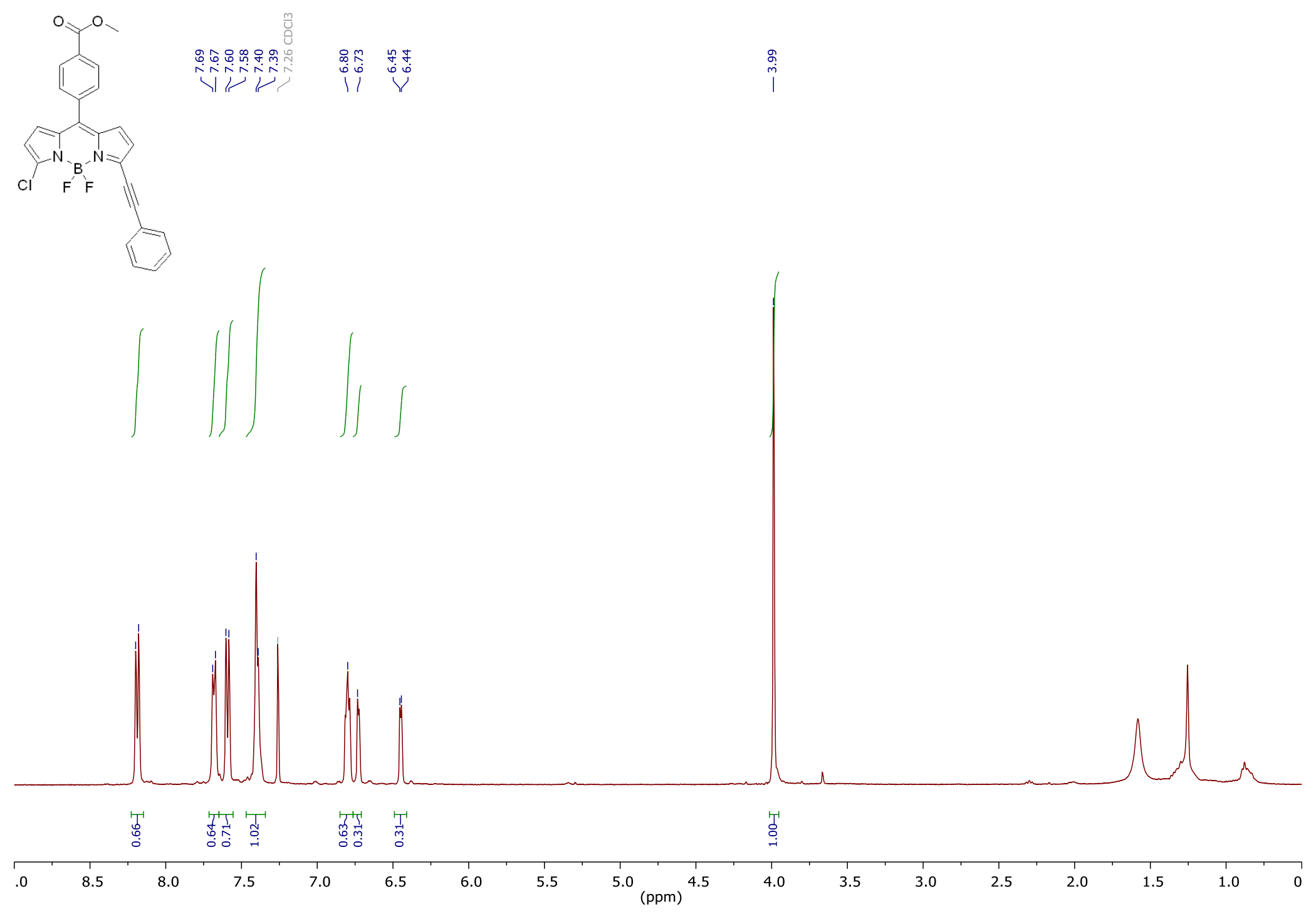


${ }^{13} \mathrm{C}$ NMR (176 MHz, CDCl 3$):$ 4-(3-chloro-5,5-difluoro-7-(phenylethynyl)-5H-4 $\lambda^{4}, 5 \lambda^{4}$-dipyrrolo[1,2-c:2',1'-f][1,3,2]diazaborinin-10yl)benzoate (8)
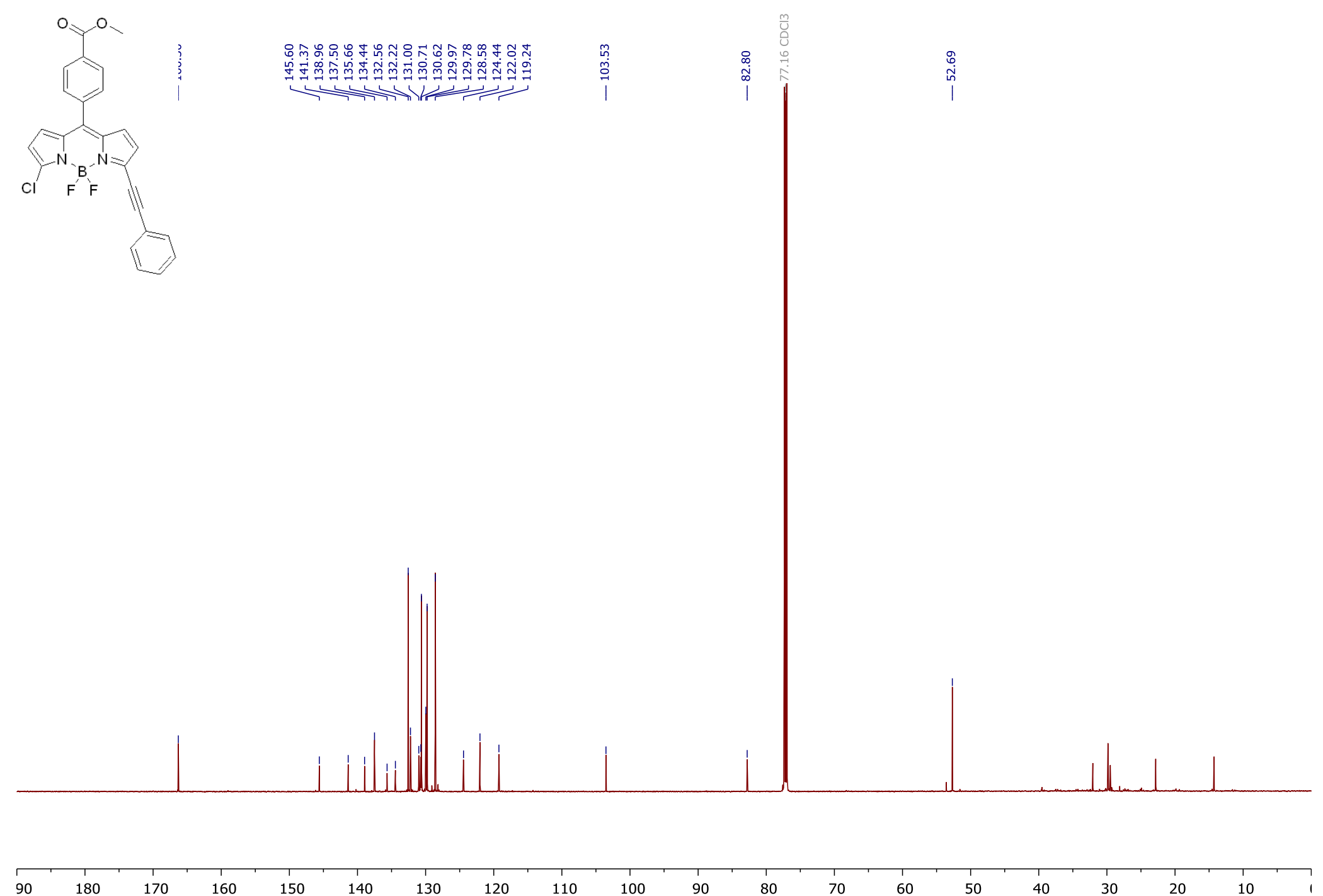

150

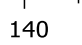

130

120

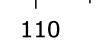

10090

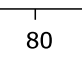

70 
${ }^{19}$ F NMR (282 MHz, CDCl 3 ): 4-(3-chloro-5,5-difluoro-7-(phenylethynyl)-5H-4 $\lambda^{4}, 5 \lambda^{4}$-dipyrrolo[1,2-c:2',1'-f][1,3,2]diazaborinin-10yl)benzoate (8)

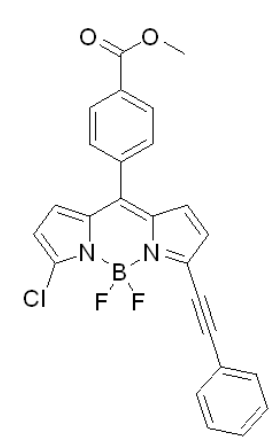

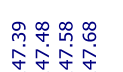

1111

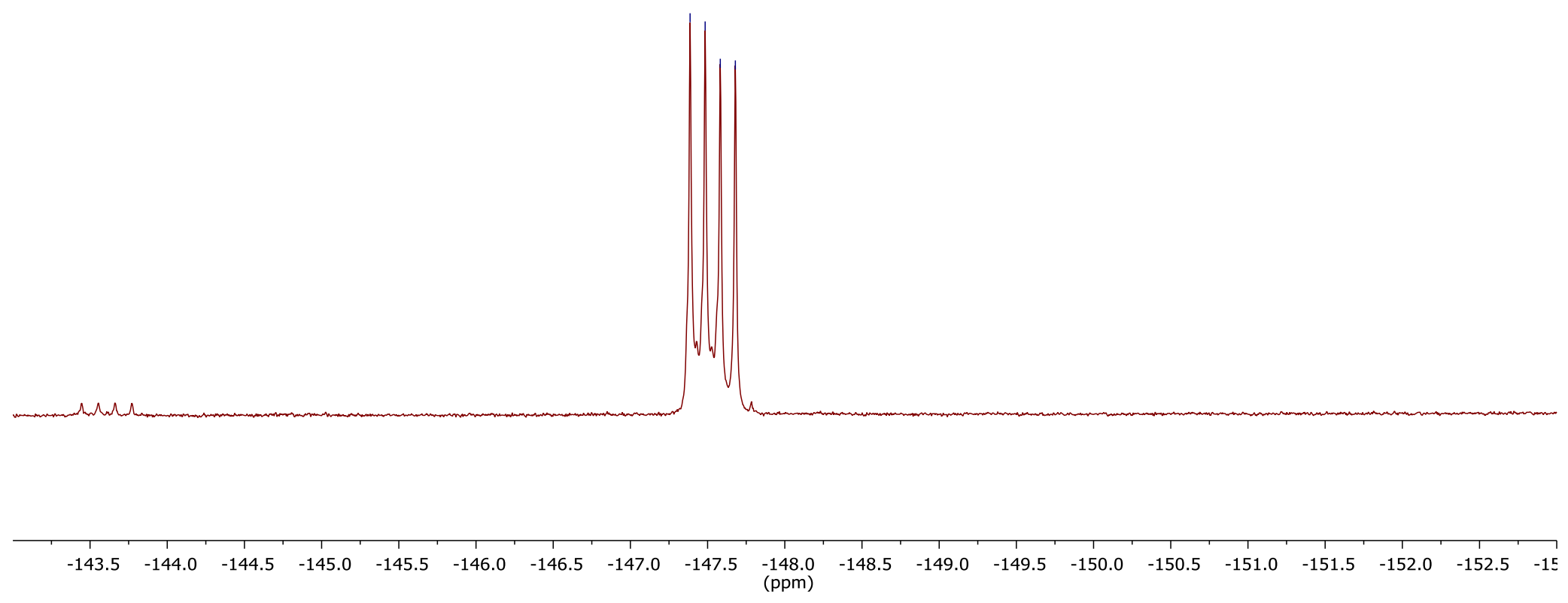


${ }^{11}$ B NMR (96 MHz, CDCl 3 ): 4-(3-chloro-5,5-difluoro-7-(phenylethynyl)-5H-4 $\lambda^{4}, 5 \lambda^{4}$-dipyrrolo[1,2-c:2',1'-f][1,3,2]diazaborinin-10yl)benzoate (8)

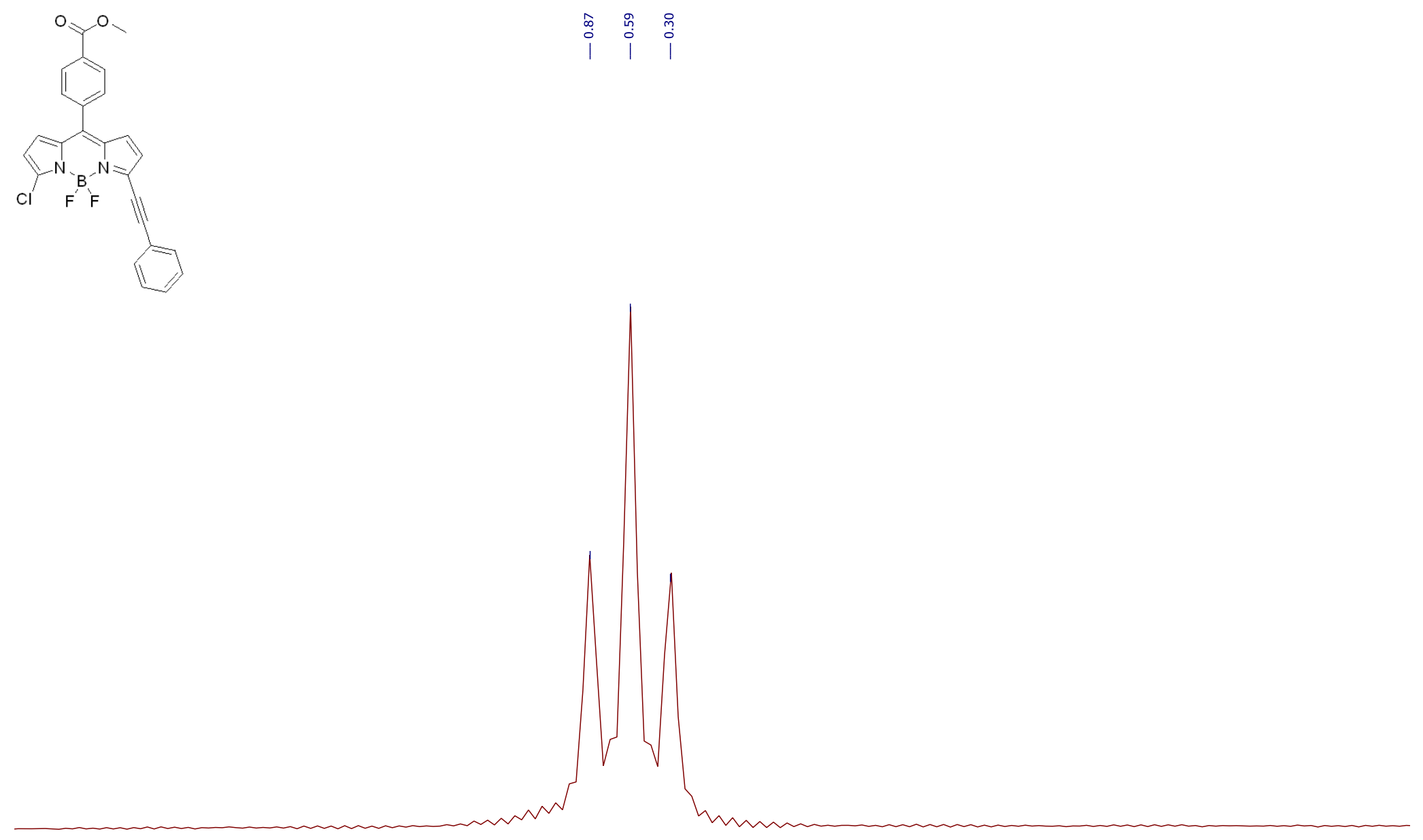


${ }^{1} \mathrm{H}$ NMR (300 MHz, CDCl 3 ): Methyl 4-(3-chloro-5,5-difluoro-7-phenyl-5H-5 $\lambda^{4}, 6 \lambda^{4}$-dipyrrolo[1,2-c:2',1'-f][1,3,2]diazaborinin-10yl)benzoate (9)

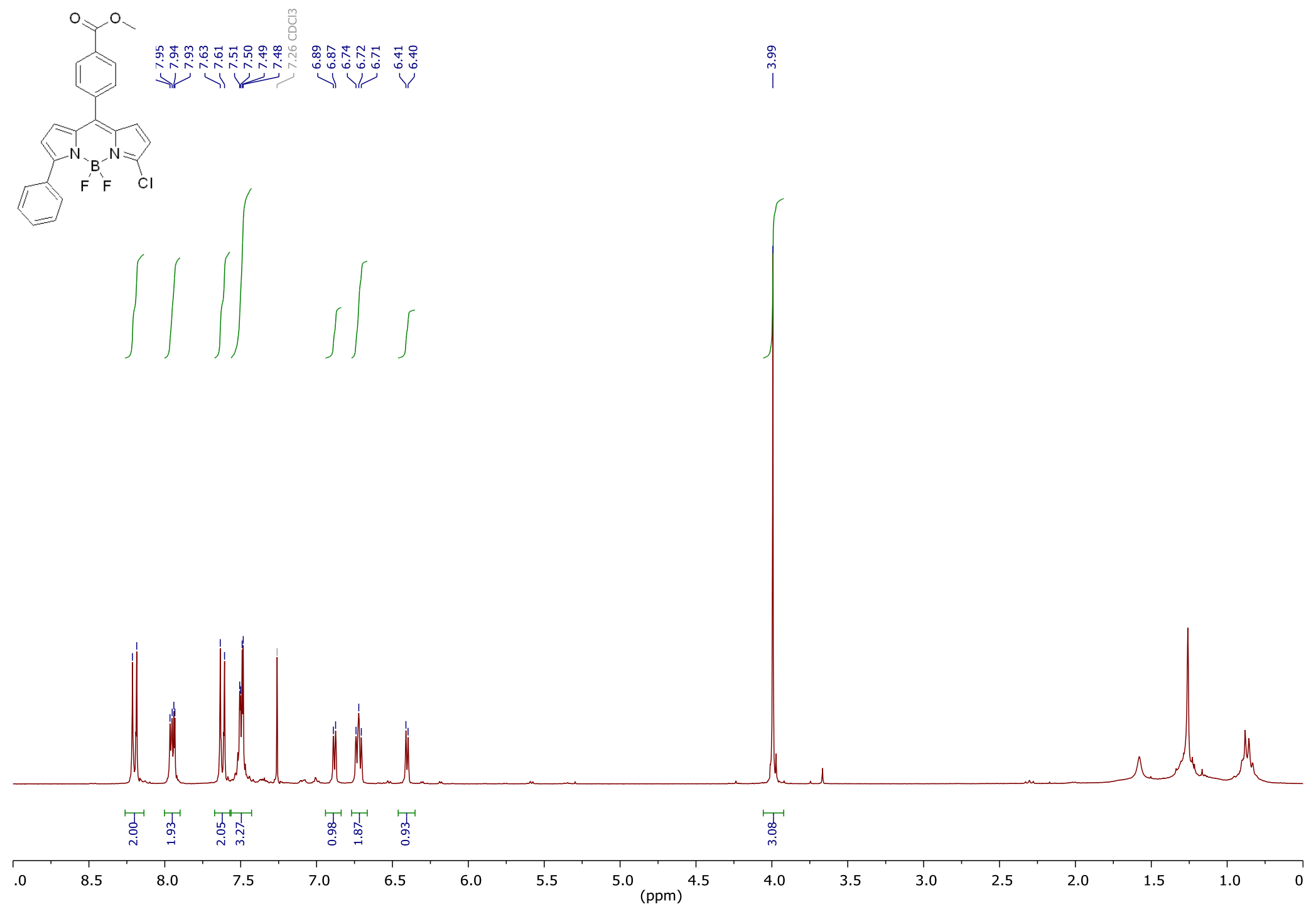


${ }^{13} \mathrm{C}$ NMR (75 MHz, CDCl 3$)$ : Methyl 4-(3-chloro-5,5-difluoro-7-phenyl-5H-5 $\lambda^{4}, 6 \lambda^{4}$-dipyrrolo[1,2-c:2',1'-f][1,3,2]diazaborinin-10yl)benzoate (9)

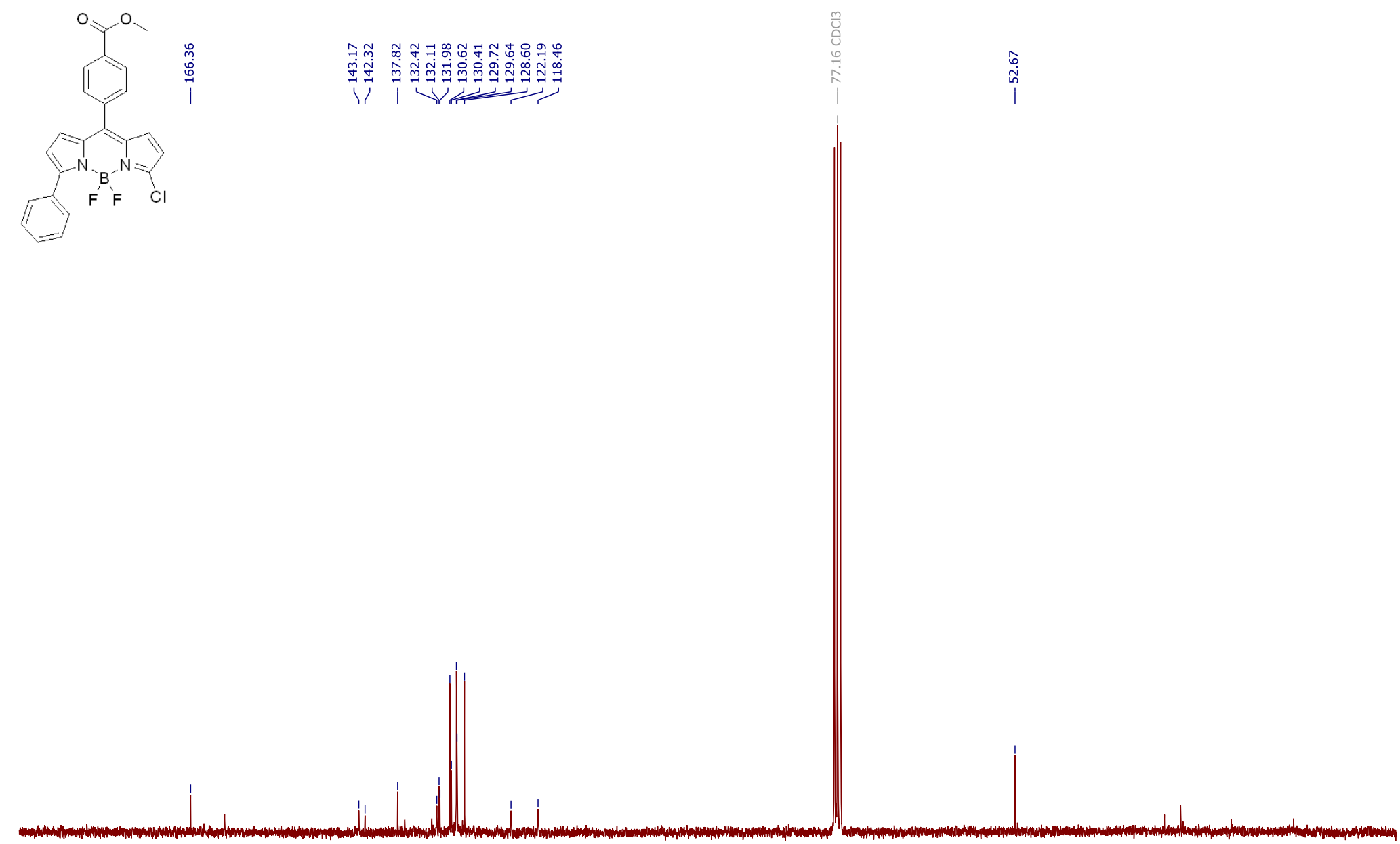


${ }^{19}$ F NMR (282 MHz, CDCl 3 ): Methyl 4-(3-chloro-5,5-difluoro-7-phenyl-5H-5 $\lambda^{4}, 6 \lambda^{4}$-dipyrrolo[1,2-c:2',1'-f][1,3,2]diazaborinin-10yl)benzoate (9)
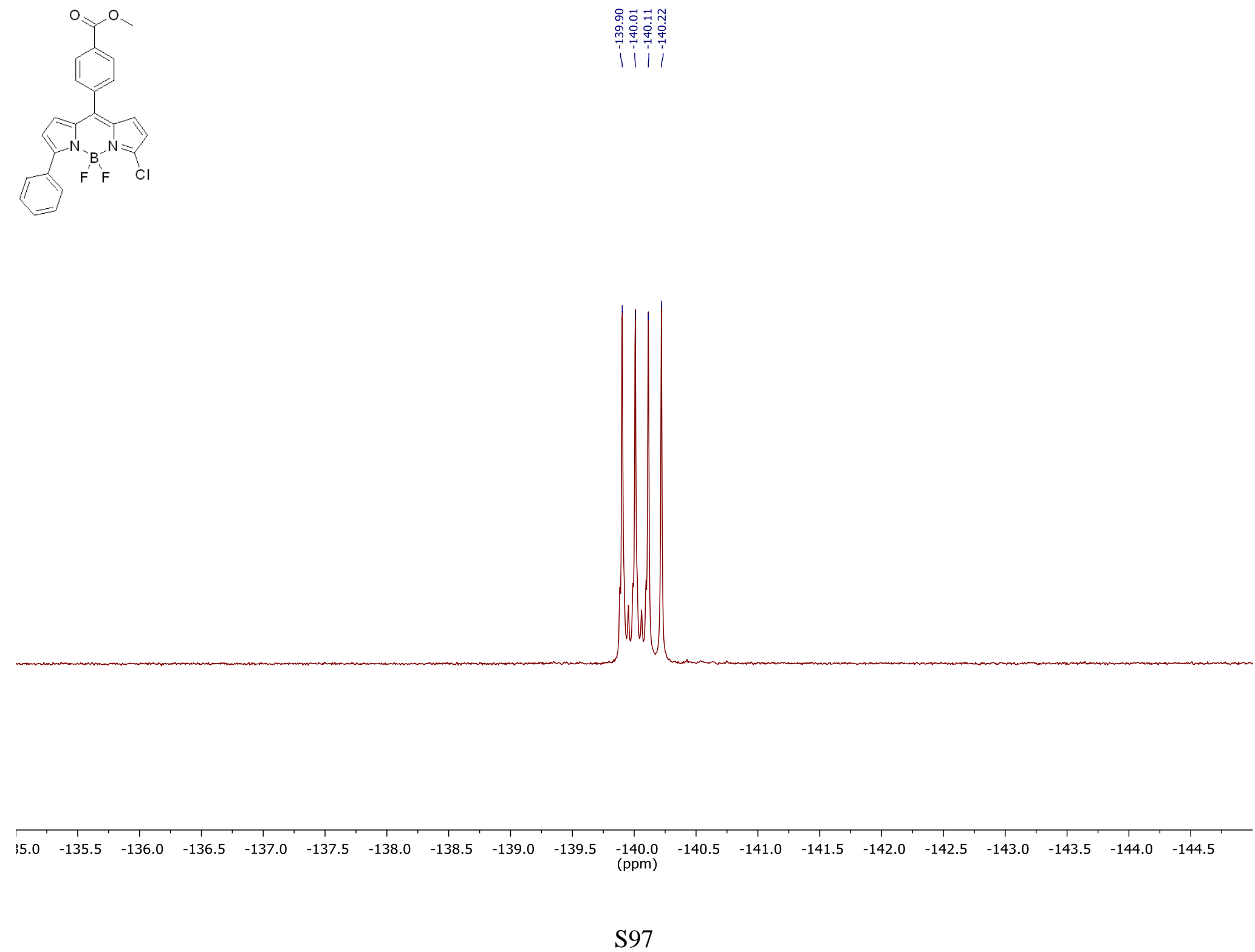
${ }^{11}$ B NMR (96 MHz, CDCl 3 ): Methyl 4-(3-chloro-5,5-difluoro-7-phenyl-5H-5 $\lambda^{4}, 6 \lambda^{4}$-dipyrrolo[1,2-c:2',1'-f][1,3,2]diazaborinin-10yl)benzoate (9)

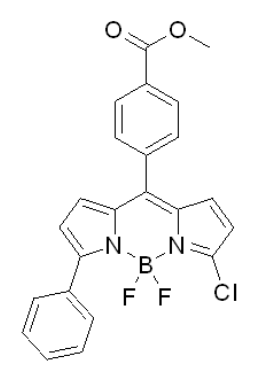

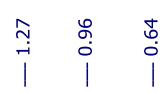

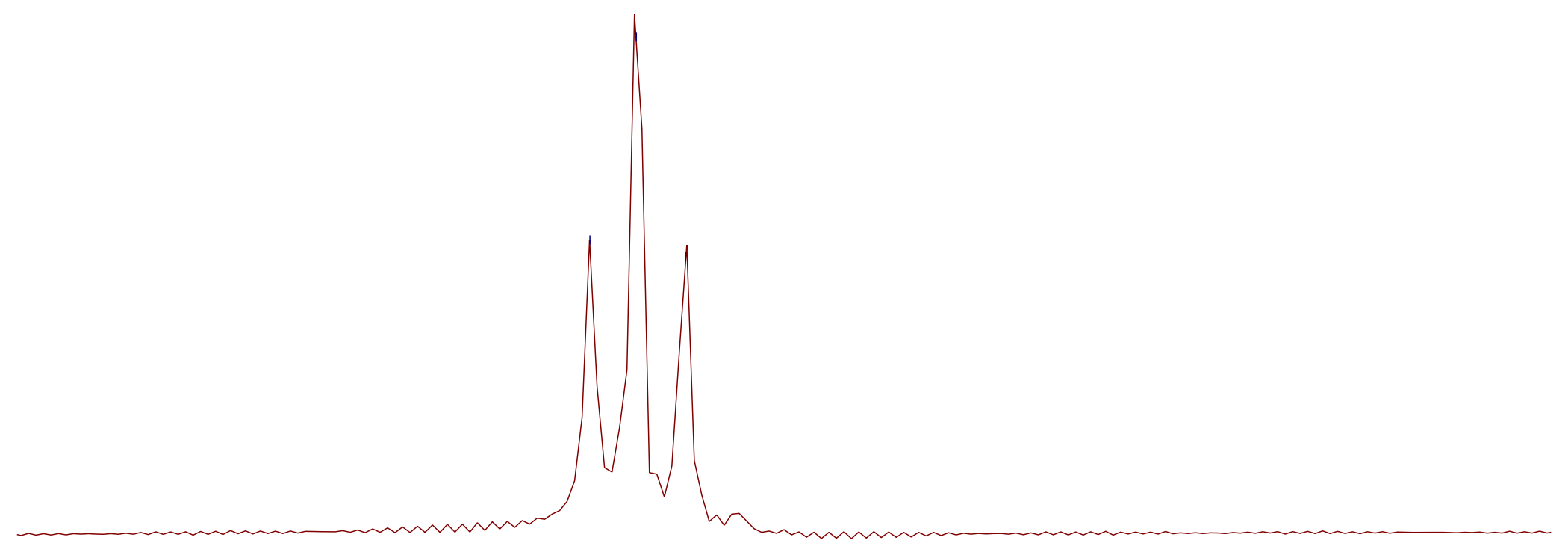

4.5 
HRMS: Methyl 4-(5,5-difluoro-3,7-diiodo-5 $H$-4 $4 \lambda^{4}, 5 \lambda^{4}$-dipyrrolo[1,2-c:2',1'-f][1,3,2] diazaborinin-10-yl)benzoate (3a)

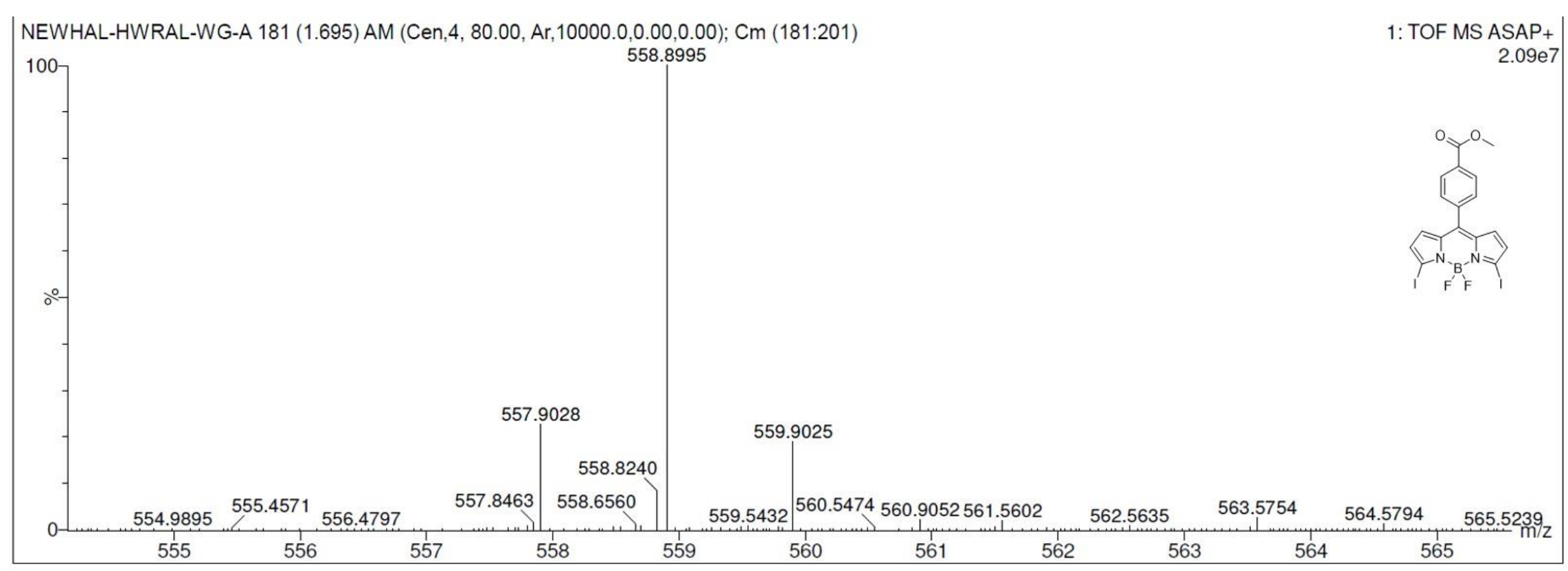


HRMS: 5,5-difluoro-3,7-diiodo-10-(3-nitrophenyl)-5H-4 $\lambda^{4}, 5 \lambda^{4}$-dipyrrolo[1,2-c:2',1'-f][1,3,2]diazaborinine (3b)

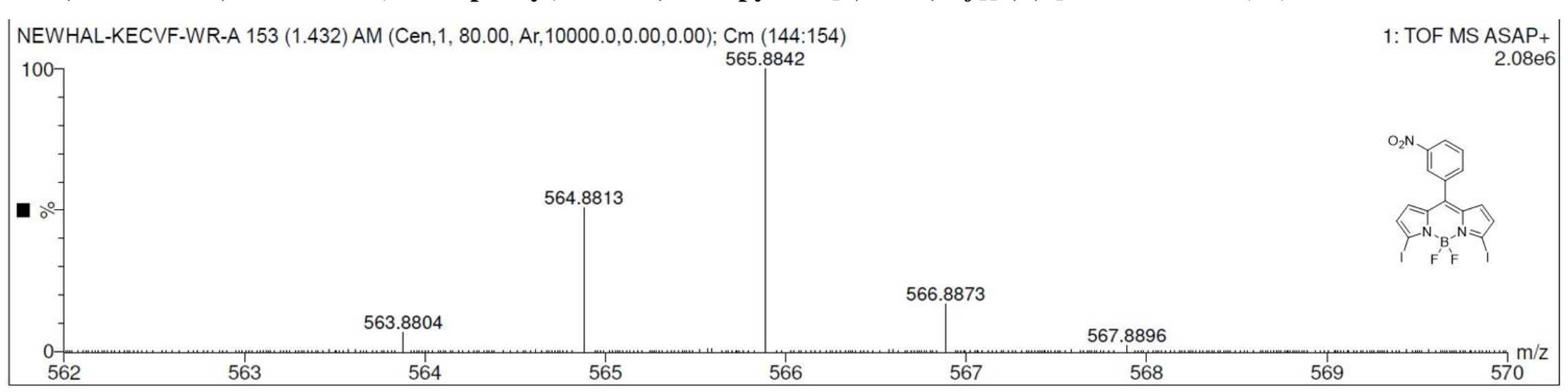

HRMS: 5,5-difluoro-3,7-diiodo-10-(4-methoxyphenyl)-5H-4 $\lambda^{4}, 5 \lambda^{4}$-dipyrrolo[1,2-c:2',1'-f][1,3,2]diazaborinine (3c)

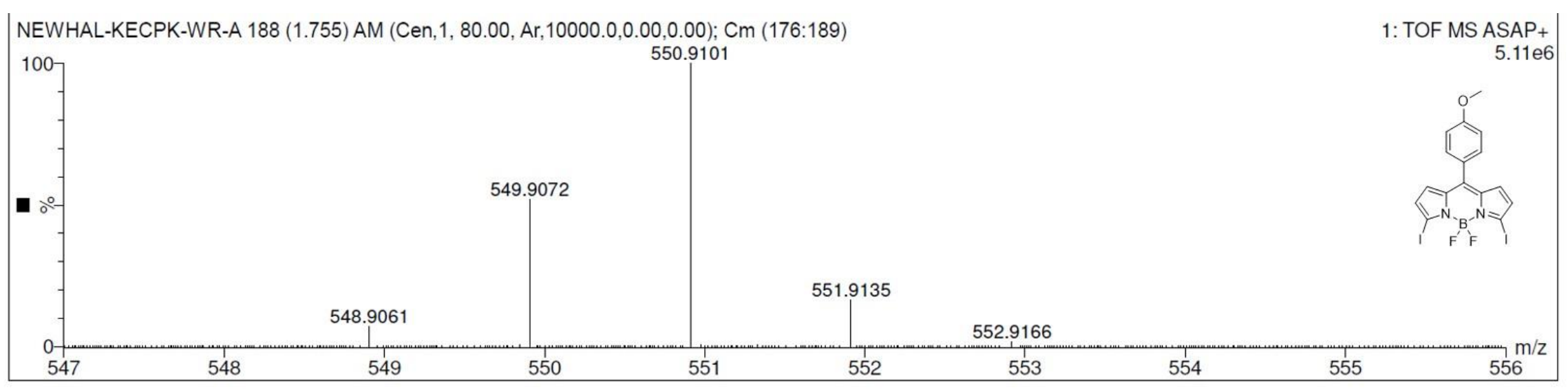


HRMS: 5,5-difluoro-3,7-diiodo-10-(3-methoxyphenyl)-5H-4 $\lambda^{4}, 5 \lambda^{4}$-dipyrrolo[1,2-c:2',1'-f][1,3,2]diazaborinine (3d)

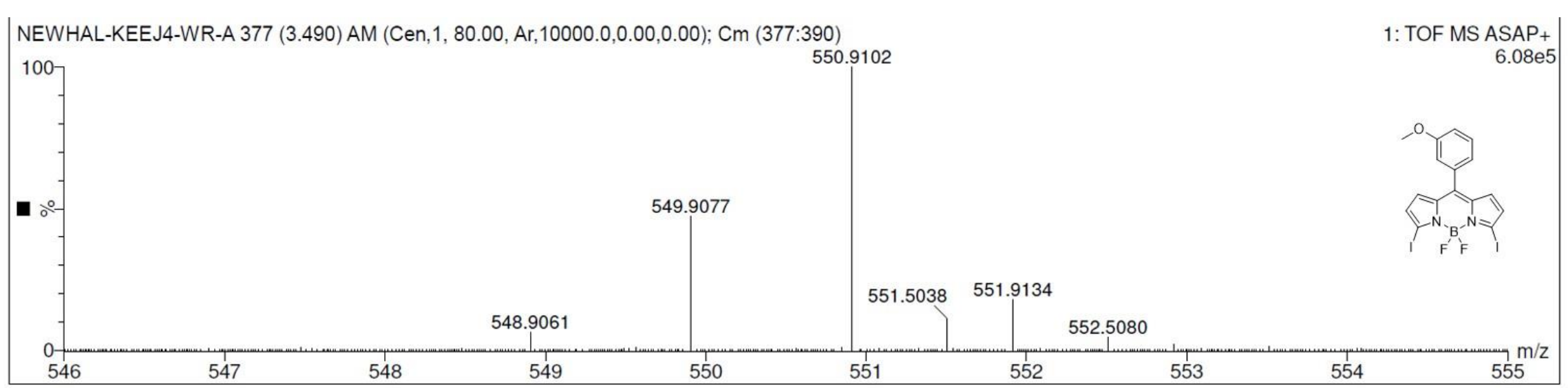

HRMS: Methyl 4-(5,5-difluoro-3,7-diphenyl-5H-4 $\lambda^{4}, 5 \lambda^{4}$-dipyrrolo[1,2-c:2',1'-f][1,3,2] diazaborinin-10-yl)benzoate (4)

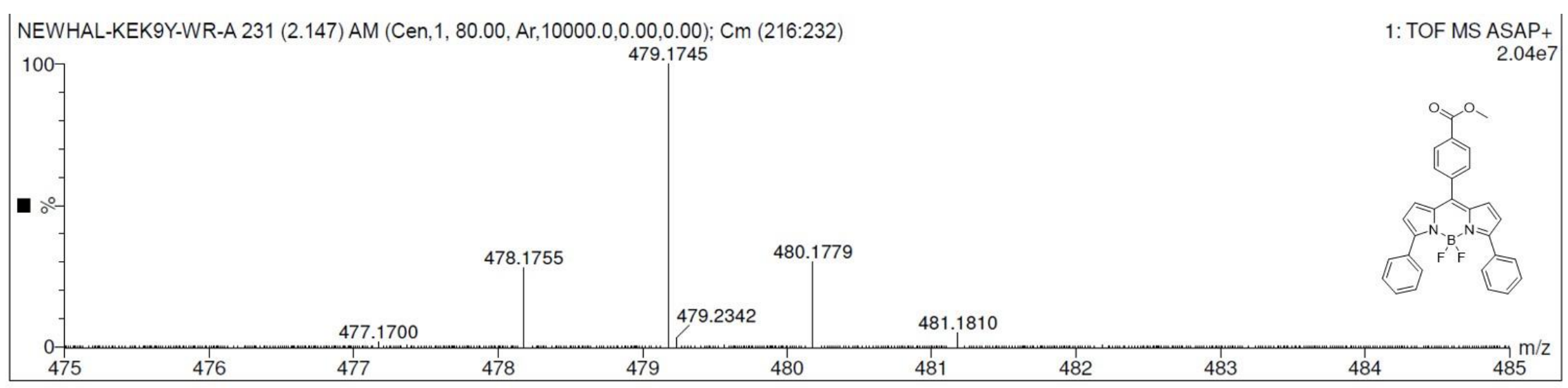


HRMS: Methyl 4-(3-butyl-5,5-difluoro-7-phenyl-5H-5 $\lambda^{4}, 6 \lambda^{4}$-dipyrrolo[1,2-c:2',1'-f][1,3,2]diazaborinin-10-yl)benzoate (5)

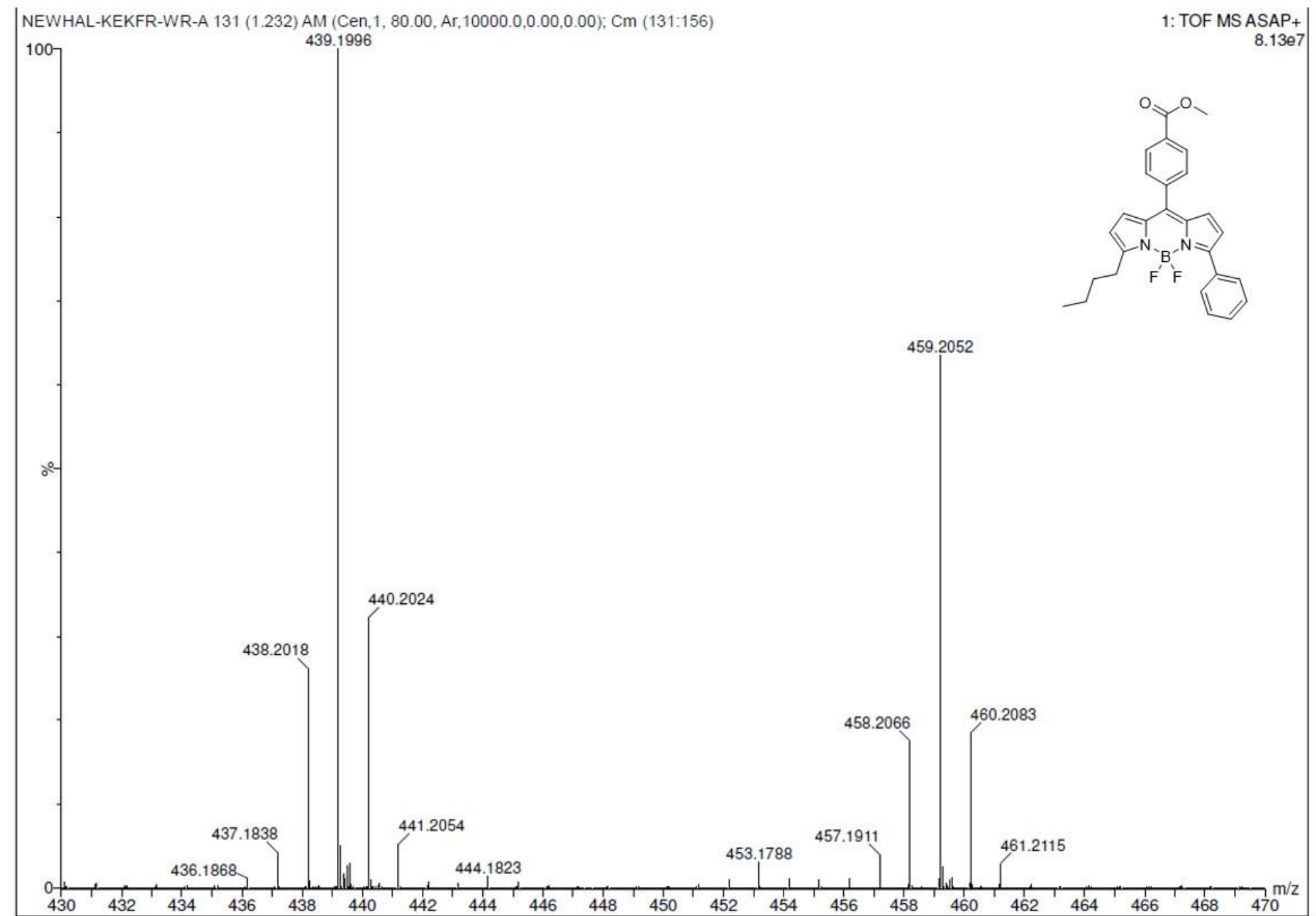


HRMS: Methyl 4-(5,5-difluoro-3,7-di((E)-styryl)-5H-4 $\lambda^{4}, 5 \lambda^{4}$-dipyrrolo[1,2-c:2',1'-f][1,3,2]diazaborinin-10-yl)benzoate (6)

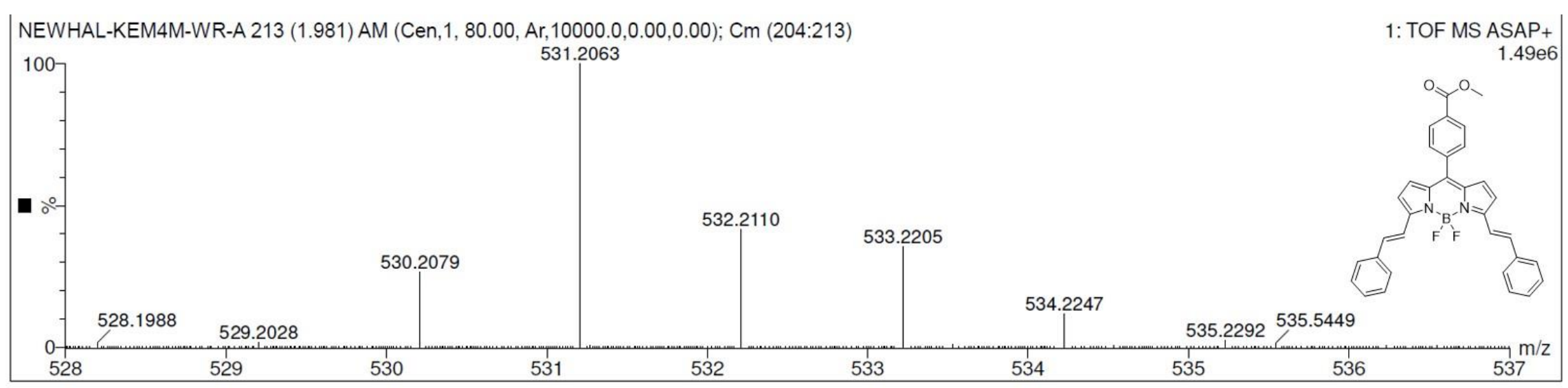

HRMS: Methyl 4-(5,5-difluoro-3,7-bis(phenylethynyl)-5H-4 $\lambda^{4}, 5 \lambda^{4}$-dipyrrolo[1,2-c:2',1'-f][1,3,2]diazaborinin-10-yl)benzoate (7)

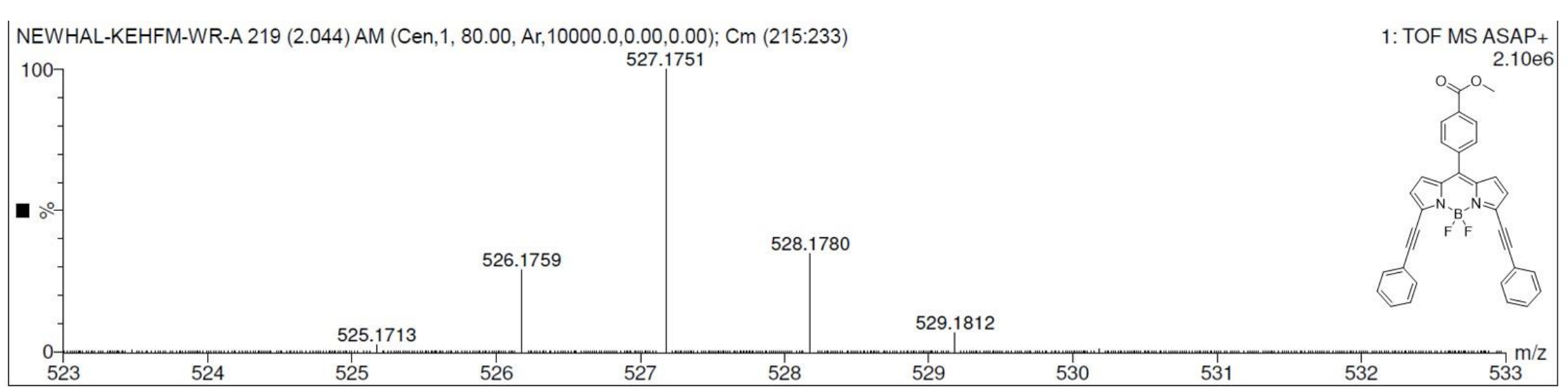


HRMS: 4-(3-chloro-5,5-difluoro-7-(phenylethynyl)-5H-4 $\lambda^{4}, 5 \lambda^{4}$-dipyrrolo[1,2- $c: 2$ ', $\left.1^{\prime}-f\right][1,3,2]$ diazaborinin-10-yl)benzoate (8)

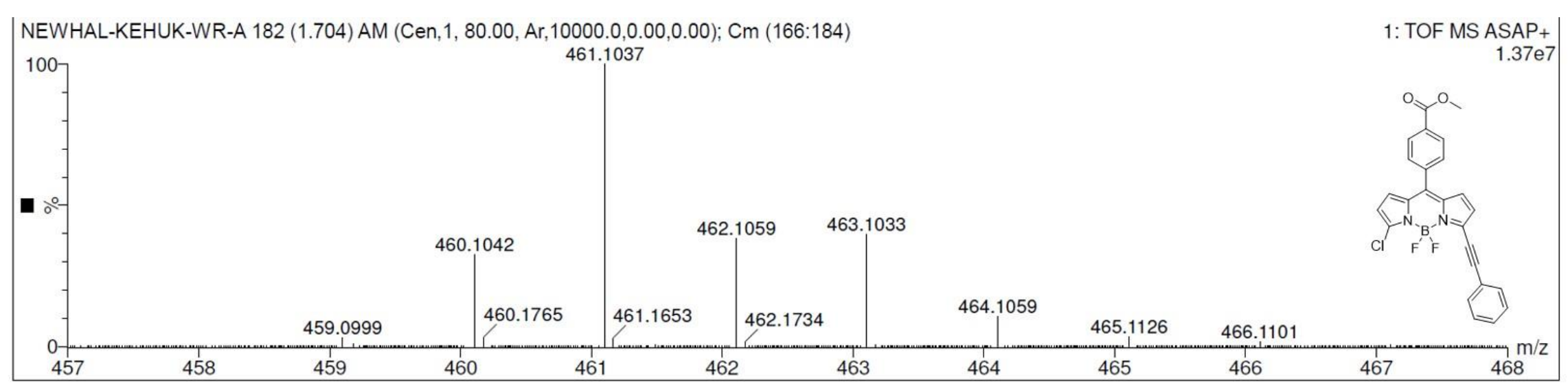

HRMS: Methyl 4-(3-chloro-5,5-difluoro-7-phenyl-5H-5 $\lambda^{4}, 6 \lambda^{4}$-dipyrrolo[1,2-c:2',1'-f][1,3,2]diazaborinin-10-yl)benzoate (9)

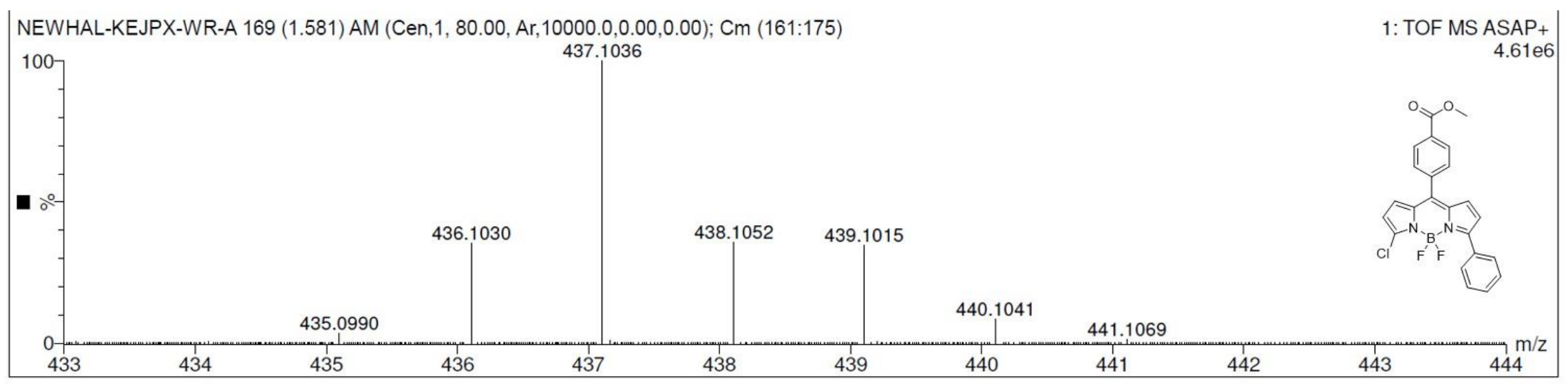

